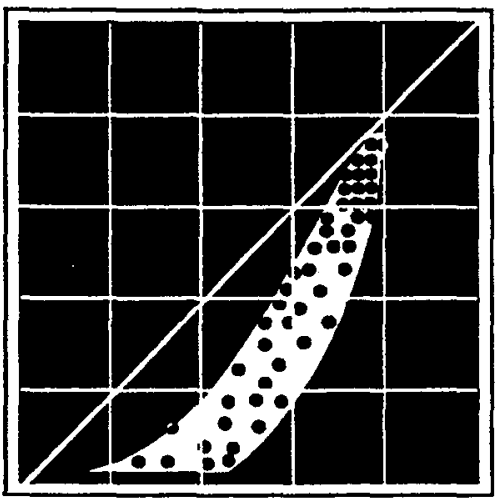

NREL/TP-463-5608

DE93018210

\title{
Users Manual for SERI QC Software Assessing the Quality of Solar Radiation Data
}

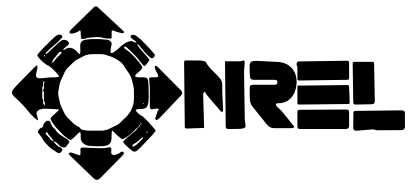

National Renewable Energy Laboratory 1617 Cole Boulevard Golden, Colorado 80401-3393

A national laboratory operated for the U.S. Department of Energy under contract No. DE-AC02-83CH10093

December 1993 


\section{NOTICE}

NOTICE: This report was prepared as an account of work sponsored by an agency of the United States government. Neither the United States governn nor any agency thereof, nor any of their employees, makes any warranty, express or implied, or assumes any legal liability or responsibility for the accurd completeness, or usefulness of any information, apparatus, product, or process disclosed, or represents that its use would not infringe privâtely owned rights. Reference herein to any specific commercial product, process, or service by trade name, trademark, manufacturer, or otherwise does not necessarily constitute or imply its endorsement, recommendation, or favoring by the United States government or any agency thereof. The views and opinions of autt $\mathrm{s}$ expressed herein do not necessarily state or reflect those of the United States government or any agency thereof.

\section{Printed in the United States of America \\ Available from: \\ National Technical Information Service \\ U.S. Department of Commerce \\ 5285 Port Royal Road \\ Springfield, VA 22161 \\ Price: Microfiche A01 \\ Printed Copy A10}

Codes are used for pricing all publications. The code is determined by the number of pages in the publication. Information pertaining to the pricing codes can be found in the current issue of the following publications which are generally available in most libraries: Energy Research Abstracts (ERA): Governmini Reports Announcements and Index (GRA and I); Scientific and Technical Abstract Reports (STAR); and publication NTIS-PR-360 available from NTIS af ${ }_{1}$ above address. 


\section{Preface}

Users Manual for SERI QC Software - Assessing the Quality of Solar Radiation Data provides general information on solar radiation measurements and quality control measures and options. It was written for the user of SERI QC, a software package developed by the Analytic Studies Division at the National Renewable Energy Laboratory (formerly the Solar Energy Research Institute) for performing quality assessment of data obtained from one-minute to one-hour integrations of solar radiation. It is not applicable for performing quality assessment of daily total solar radiation energy.

SERI QC is described from several aspects, and the theory encompassing this approach to postmeasurement quality assessment is discussed. The utility of this software package is illustrated through examples of typical measurement problems.

Work on this manual was sponsored by the U.S. Department of Energy's Resource Assessment Program managed by the Photovoltaics Technology Division of the Office of Energy Efficiency and Renewable Energy.

During the past five years, many people have contributed to the development of SERI QC and this users manual. The concepts were developed by Gene Maxwell, Tom Stoffel, Martin Rymes, and Daryl Myers. The original VAX computer programs were written by Martin Rymes with assistance from Tom Stoffel and Steve Wilcox. All of these people contributed to the development of boundaries and limits defining the expected range of solar radiation values for individual stations and months. The PC version of the code was written by Martin Rymes, Steve Wilcox, and Nancy Birkenheuer. The manual was written by Gene Maxwell, Steve Wilcox, and Martin Rymes.

Approved for the

NATIONAL RENEWABLE ENERGY LABORATORY

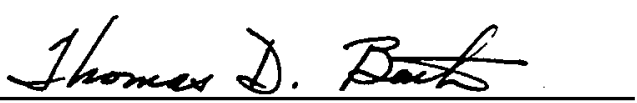

Thomas D. Bath, Director

Analytic Studies Division

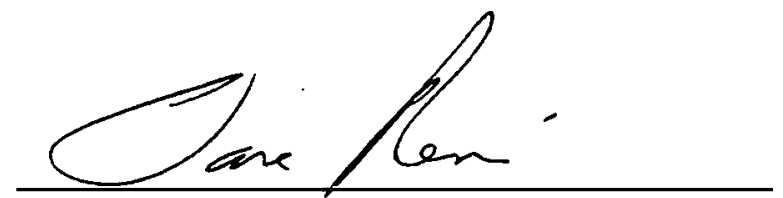

Dave Renné, Leader

Solar Radiation Resource Assessment Project

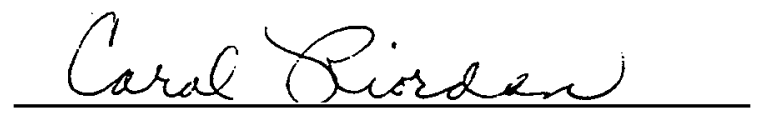

Carol Riordan, Manager

Technology \& Resource Assessment Branch 


\section{Table of Contents}

Welcome to SERI QC ...............................................................................................

Part 1: Technical Information......................................................................

Chapter 1- Introduction ..................................................................... 5

Chapter 2 - Solar Radiation Measurements ....................................... 7

Chapter 3- Quality Control Measures and Options............................. 15

Chapter 4 - Conceptual Description of SERI QC ............................. 19

Chapter 5- Quality Assessment Boundaries ...................................... 33

Chapter 6 - A Comprehensive Quality Assessment System ............. 41

Part 2: Users Guide ...................................................................................... 51

Getting Started ............................................................................. 53

Chapter 7 - The SERI QC Software and Hardware Environment ..... 83

Chapter 8 - QCFIT Software Users Guide.......................................... 97

Chapter 9 - SERI_QC1 Software Users Guide .................................. 145

Appendix QCFIT Plots for 12 NOAA Stations ...................................................... 161 


\section{List of Figures}

2-1 Examples of solar radiometers....................................................... 8

2-2 Instruments used to measure diffuse solar radiation............................ 12

4-1 K-space scatter plot of all solar radiation data collected at

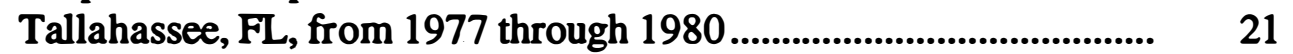

4-2 K-space scatter plot of medium air mass (1.25 - 2.5) solar radiation data collected at Tallahassee, FL, from 1977 through 1980 .

4-3 K-space scatter plot of high air mass (2.5 - 5.8) solar radiation data collected at Tallahassee, FL, from 1977 through 1980

4-4 K-space scatter plot of all solar radiation data collected at Tallahassee, FL, from 1977 through 1980, with added interpretive information

4-5 K-space scatter plots of solar radiation data for three air mass ranges, for Nashville, TN, during April...

4-6 Regions in K-space that correspond to known atmospheric conditions....

4-7 K-space plots for Los Angeles, CA, and Lander, WY, illustrating cloud-climate effect

4-8 K-space plots for Fresno, CA, Montogomery, AL, and Boise, ID, illustrating cloud-climate effects.

5-1 Gompertz curves, illustrating the effects of each of the four coefficients

5-2 Set of six Gompertz curves used to define the shape of the left boundary of $\mathrm{K}$-space plots of solar radiation data.

5-3 Set of five Gompertz curves used to define the shape of the right boundary of $\mathrm{K}$-space plots of solar radiation data.

5-4 Family of Gompertz curves used to define left boundary shape number 1

5-5 Global-direct and global-diffuse scatter plots for Bismarck, ND, for July

5-6 An illustration of the effects of integration times on K-space scatter plots. Data are from San Antonio, TX, during July

6-1 Chart showing the flow of data through SERI_QC1 


\section{List of Figures (continued)}

GS-1. Sample data file............................................................................... 56

GS-2. Initial Low air mass boundary fit by QCFIT ...................................... 62

GS-3. Making wider boundaries with the (L)ess err option............................. 64

GS-4. Moving the left boundary with (N)arrow............................................. 65

GS-5. Setting the right curve and K maximums with (S)elect........................ 68

GS-6. Initial Medium air mass curve placement ............................................. $\quad 70$

GS-7. Medium air mass after adjusting curves ............................................... 72

GS-8. Initial curve placement for High air mass ............................................. 73

GS-9. New right curve after using (S)elect ...................................................... 75

GS-10. A steeper right curve using (S)elect....................................................... 76

7-1 Annotated sample QC-zero file ........................................................ 87

7-2 SOLPOS subroutine ............................................................... 92

8-1 Flow diagram for using QCFIT ...................................................... 108

8-2 PC QCFIT editing screen................................................................. 116

8-3 VAX QCFIT editing screen ....................................................... 116

8-4 QCFIT screen information boxes...................................................... 117

8-5 Scatter plot of original data for low air mass for July at Eugene, OR .................................................................................... 133

8-6 Scatter plot of original data for high air mass for July at Eugene, OR ....................................................................................... 133

8-7 Scatter plot of data in Figure 8-6 modified to simulate a sensor tilted $2^{\circ}$ to the south.............................................................. 133

8-8 Scatter plot of data in Figure 8-6 modified to simulate a sensor tilted $2^{\circ}$ to the east...

8-9 Scatter plot of data in Figure 8-5 modified to simulate a sensor tilted $5^{\circ}$ to the south............................................................... 135

8-10 Scatter plot of data in Figure 8-6 modified to simulate a sensor tilted $5^{\circ}$ to the south.

8-11 Scatter plot of data in Figure 8-5 modified to simulate a sensor tilted $5^{\circ}$ to the east

8-12 Scatter plot of data in Figure 8-6 modified to simulate a sensor tilted $5^{\circ}$ to the east

8-13 Scatter plot of data in Figure 8-6 modified to simulate a $1^{\circ}$ error in longitude or a 4 -minute error in time.

8-14 Scatter plot of data in Figure 8-6 modified to simulate a $4^{0}$ error in longitude or a 16-minute error in time.

8-15 Scatter plot of data from Figure 8-5 modified to simulate a $10^{\circ}$ error in latitude.

8-16 Scatter plot of data from Figure 8-6 modified to simulate a $10^{\circ}$ error in latitude. 


\section{List of Figures (concluded)}

8-17 Scatter plot of data in Figure 8-5 modified to simulate the effects of miscalibrating the pyranometer by minus $5 \%$

8-18 Scatter plot of data in Figure 8-5 modified to simulate the effects of miscalibrating the pyranometer by plus $5 \%$

8-19 Scatter plot of data in Figure 8-5 modified to simulate the effects of miscalibrating the pyrheliometer by minus $5 \%$.

8-20 Scatter plot of data in Figure 8-6 modified to simulate the effects of miscalibrating the pyrheliometer by minus $5 \%$

8-21 Scatter plot of data in Figure 8-5 modified to simulate miscalibrating both the pyranometer and pyrheliometer by minus $5 \%$

8-22 Scatter plot of data in Figure 8-5 modified to simulate miscalibrating the pyranometer by minus $5 \%$ and the pyrheliometer by plus $5 \%$

8-23 Scatter plot of global-diffuse data for low air mass for June at Albuquerque modified to simulate a bad shadowband adjustment ...

8-24 Scatter plot of unmodified global-direct data for Albuquerque shown in Figure 8-23

8-25 Scatter plot of global-direct data for high air mass for November at Bluefield, WV. The widely scattered data points are probably due to effects of snow and frost.

8-26 Scatter plot of global-diffuse data for high air mass for November at Bluefield, WV. A comparison of this figure with Figure 8-25 adds support to the supposition that these data were affected by snow and frost

9-1 Sample calling program

9-2 Report generated for Albuquerque, NM 151

9-3 Chart showing the flow of data through SERI_QC1 


\section{List of Tables}

4-1 Air Mass/Zenith Angle Ranges......................................................... 25

6-1 QC-zero File for Albuquerque, NM.................................................. 43

6-2 Flagging Convention for SERI_QC1 .................................................. 47

7-1 VAX Memory and Disk Requirements.............................................. 84

7-2 PC Memory and Disk Requirements .................................................. 85

7-3 Description of QC-zero File.............................................................. 88

8-1 Default QCFIT Input Data Description ............................................ 101

8-2 Variable Names...................................................................... 103

8-3 Solar Radiation Units................................................................... 103

8-4 Log-Based Integration Times and Designation .................................. 109

9-1 Measurement Unit Conversion ......................................................... 146

9-2 Input Variable Descriptions .................................................................. 147

9-3 Output Variable Descriptions ....................................................... 147 


\section{Acronyms and Nomenclature}

ETR

ETRN

IPCs

NOAA

NREL

QCFIT

SERI QC

SERI QC Users Manual

SERI_QC1

SOLZEN

WMO

WRR extraterrestrial radiation

extraterrestrial radiation normal

International Pyrheliometer Comparisons

National Oceanic and Atmospheric Adminstration

National Renewable Energy Laboratory

computer program for defining expected boundaries

NREL's quality assessment software package ${ }^{1}$

Users Manual for SERI QC Software - Assessing the Quality of Solar Radiation Data

computer subroutine for assessing data quality

solar zenith angle

World Meteorological Organization

World Radiometric Reference

1. This software package was developed when NREL was known as the Solar Energy Research Institute (SERI). Because it had been referred to as SERI QC in other documents, this name was retained. 


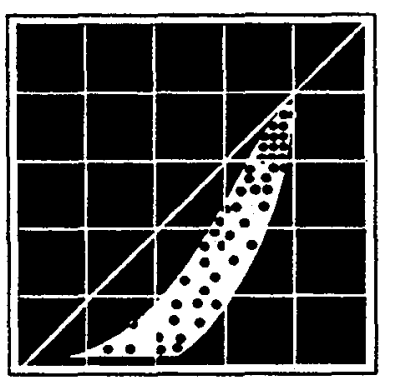

\section{Welcome To SERI QC}

SERI QC is a mathematical software package that assesses the quality of solar radiation data. This software was developed by the National Renewable Energy Laboratory when the Laboratory was called the Solar Energy Research Institute (SERI), hence the name "SERI QC." This manual contains all of the information required to assess the quality of global horizontal, direct normal, and diffuse horizontal solar radiation measurements using SERI QC software.

Serious users should be familiar with all sections of this manual. It is divided into two parts: Part 1: Technical Information and Part 2: Users Guide.

Part 1 explains solar radiation measurements, sources of error in such measurements, natural phenomena affecting solar radiation data, and the problems associated with assessing the quality of these data. Part 1 also provides a conceptual description and a comprehensive overview of the SERI QC software.

\section{Part 1: Technical Information}

Chapter 1- Introduction: Explains the concepts of quality assessment, data flagging, and two types of errors.

Chapter 2 - Solar Radiation Measurements: Introduces the methods and instrumentation of solar radiation measurements and the typical sources of measurement errors.

Chapter 3 - Quality Control Measures and Options: Contrasts quality control and quality assessment, and considers several options that have been used in the past.

Chapter 4 - Conceptual Description of SERI QC: Discusses the theory of SERI QC, defines the K-space within which SERI QC operates, and examines the expected bounds and the theoretical and empirical bounds for solar radiation data.

Chapter 5 - Quality Assessment Boundaries: Discusses the methods used to establish SERI QC limits and boundaries, including an introduction to the Gompertz function.

Chapter 6 - A Comprehensive Quality Assessment System: Provides an overview of SERI_QCl and QCFIT, the programs that form SERI QC. 


\section{Part 2: Users Guide}

Getting Started: A brief tutorial on installing and using the SERI QC software. Now that you have familiarized yourself with the capabilities of SERI QC, this section will lead you through the mechanics of installing the software and a sample session for using the software.

Chapter 7 - The SERI QC Software and Hardware Environment: Outlines SERI QC hardware and software requirements and describes the solar geometry routines used by SERI QC.

Chapter 8 - QCFIT Software Users Guide: The reference manual for the QCFIT boundary fitting program. Section 8.7 illustrates the value of QCFIT as a diagnostic tool to detect and identify measurement problems.

Chapter 9 - SERI_QC1 Software Users Guide: The reference manual for using the SERI_QC1 quality assessment subroutine.

Appendix: Contains QCFIT plots for representative locations in the United States. 


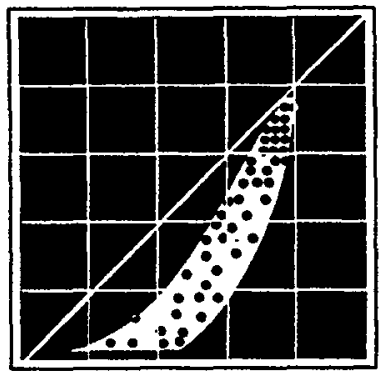

Part 1

\section{Technical}

Information

\section{Contents}

Chapter 1 - Introduction ........................................................................................

Chapter 2 - Solar Radiation Measurements ...................................................... 7

Chapter 3 - Quality Control Measures and Options........................................... 15

Chapter 4 - Conceptual Description of SERI QC ............................................. 19

4.1 Theoretical Limits.............................................................. 22

4.2 Empirical Limits ............................................................... 24

4.2.1 The Effects of Clouds .............................................. 24

4.2.2 Air Mass Effects ....................................................... 25

4.2.3 Atmospheric Effects................................................... 25

4.2.4 Climate and Seasonal Effects...................................... 28

$4.3 \quad$ References........................................................................... 32

Chapter 5 - Quality Assessment Boundaries ................................................. 33

$5.1 \quad$ The Gompertz Function ...................................................... 33

5.2 Boundaries for Other Pairs of Components ............................ 35

$5.3 \quad$ Boundaries for Other Integration Times ................................ 38

$5.4 \quad$ Summary Comments.......................................................... 40

$5.5 \quad$ References...................................................................... 40

Chapter 6 - A Comprehensive Quality Assessment System ............................. 41

$6.1 \quad$ Establishing Limits and Boundaries .......................................... 41

6.2 Designating Limits and Boundaries......................................... 42

6.3 SERI_QC1 Flow Diagram ................................................... 44

$6.4 \quad$ Tests and Flags ....................................................................... 46

6.4.1 One-Component Tests.................................................... 46

6.4.2 Two-Component Tests ................................................ 48

6.4.3 Three-Component Tests ........................................... 49

$6.5 \quad$ References..................................................................... 50 


\section{Overview}

Part 1 of this manual will probably not help you use the SERI QC software. Nevertheless, it is more important to read all of Part 1 than Part 2. Like most manuals, it is possible to exercise SERI QC without reading all of the step-by-step procedures described in Part 2.

However, similar to most mathematical software packages, it is important that you understand the theory behind the functions that the software performs.

When developing a statistical software package, it is usually assumed that the user understands statistics and, therefore, knows when and how to apply the various routines contained in the package. Statistical theory is usually not included in the manual for such a package. However, we could not assume that users of SERI QC would be adequately informed regarding solar radiation measurements, sources of error in such measurements, natural phenomena affecting solar radiation data, and the problems associated with assessing the quality of such data. Therefore, Part 1 has been written to provide a brief tutorial on these subjects. Part 1 also provides a conceptual description and a comprehensive overview of SERI QC.

We strongly recommend that you take the two or three hours you will need to read Part 1 before you start using SERI QC. If you are familiar with solar radiation measurements, you can probably reduce that time considerably by skimming Part 1 to find those topics of interest. Combined with Section 8.7 in Part 2, Part 1 will provide the understanding needed to interpret the results you will obtain from SERI QC. 


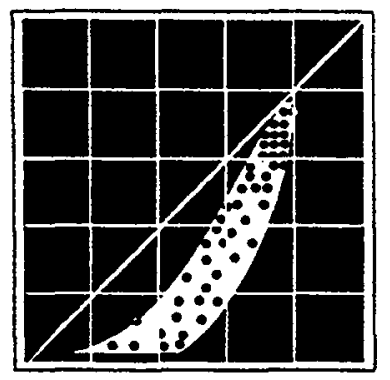

\section{Chapter 1}

\section{Introduction}

This manual describes the procedures and software for assessing the quality of solar radiation data. This does not constitute quality control because quality control must take place during the preparations for data collection (selection, calibration, and installation of instruments), during the measurement process, and during the transmission (if any) and recording of the numerical values. Once the data are recorded, only quality assessment can be performed.

If quality assessment is performed in real time or soon after the measurement process is completed, it can provide input to control the quality of future measurements. Furthermore, quality assessment can be used for quality control if data judged to be bad are deleted and/or modified. We do not subscribe to these actions because the deletion or modification of data destroys information that might be useful to the user. For example, if an instrument has gone through a gradual failure and all of the data that fail quality assessment criteria are deleted or modified, the user of the data may not be able to detect what was happening and will not question the accuracy of other data collected before the instrument completely failed.

Therefore, the SERI QC procedures and software do not delete or modify data. Instead, flags are set to inform the user of any departure of the data from expected values. These flags indicate the magnitude and direction of such departures.

For the flags to communicate as much information as possible, this manual attempts to identify and explain the probable causes of various flags. However, we cannot overemphasize the following:

Flags only indicate that data do or do not fall within expected ranges. This does not mean that the data are or are not valid. 
A flag that indicates data are outside the expected ranges can be the result of one or more of the following causes:

- The data are in error due to one or more sources of error

- The ranges of expected values have been erroneously set or are not appropriate for the specific conditions under which the data were collected

- The data may have been affected by short-lived phenomena that are not accounted for by the expected ranges.

Similarly, a flag that indicates that data fall within expected ranges can mean any of the following:

- The data are correct and are of good quality.

- The expected ranges are so broad that the data can be in error and still fall within the range of acceptance.

- The expected ranges have been erroneously set, leading to the acceptance of bad data.

Given these possible interpretations of flags, we recommend that expected ranges be set such that a small percentage (approximately 5\%) of Type 1 errors (flagging good data as bad) are produced. This should minimize, as much as possible, the occurrence of Type 2 errors (flagging bad data as good). 


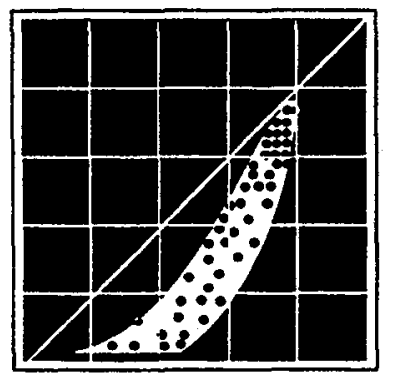

\section{Chapter 2 \\ Solar Radiation Measurements}

Solar radiation is electromagnetic radiation emitted by the sun. Approximately 99\% of the solar radiation incident on the earth's surface is encompassed within the wavelength range from $0.3 \mu \mathrm{m}$ to $3.0 \mu \mathrm{m}$. Quantifying the solar power or energy incident on the earth's surface is one of the more difficult field measurements. The measurement must be made through an energy conversion process whereby electromagnetic radiant energy is converted into another form of energy that can be measured by an available device. Most commonly, the radiant energy is first converted into thermal energy using a black absorbing surface, then into electrical energy using a thermopile, whose output is measured with a voltmeter.

Photoelectric instruments convert radiant energy (photons) directly into electrical energy.

Another very common device used to measure solar energy employs a bimetallic strip (two metals having different coefficients of expansion). The strip bends when heated by the sun's rays (conversions from radiant to thermal to mechanical energy) and moves a pen across the paper of a strip chart recorder. The deflection of the pen provides a measure of the intensity of the radiant solar energy. For example, Robitsch actinometers are still in use today in many countries around the world. Examples of all three of these solar radiometers are shown in Figure 2-1.

As shown in Figure 2-1, pyranometers are solar instruments with a $180^{\circ}$ field of view, designed to measure the global or total radiation coming from the celestial sphere above the surface of the earth. Pyrheliometers are instruments with a $5.7^{\circ}$ field of view designed to measure the radiation coming from the solar disc and the immediate region around the sun (circumsolar). The pyrheliometer must be employed with a tracking device to keep it pointed at the sun.

All of the instruments used to measure solar radiation exhibit features affecting the accuracy of the measurements. A problem common to all pyranometers is the effect of the position of the sun relative to the plane of the instrument. Ideally, the energy absorbed by the sensing device and the response of that device should vary according to the cosine of the angle of incidence of the solar radiation. When measuring global horizontal radiation the angle of incidence becomes the solar zenith angle. The most common instruments fail to follow this cosine law and produce significant errors at solar zenith angles greater than $60^{\circ}$. In the future, it is possible that this error may be reduced to a negligible value. It is important to 


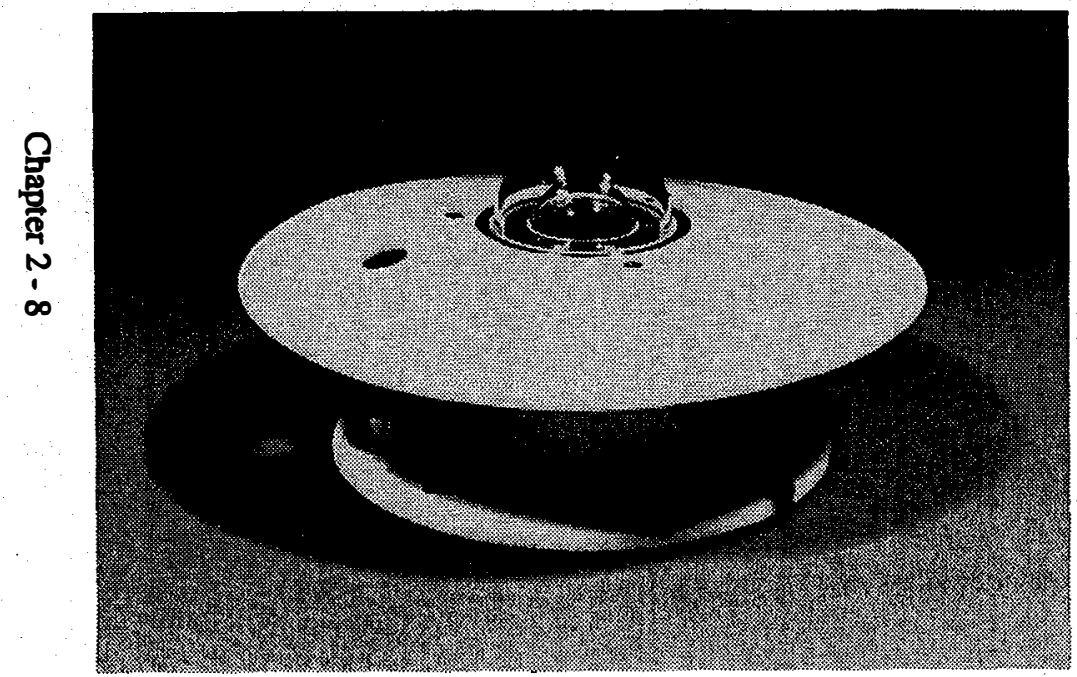

Thermopile pyranometer

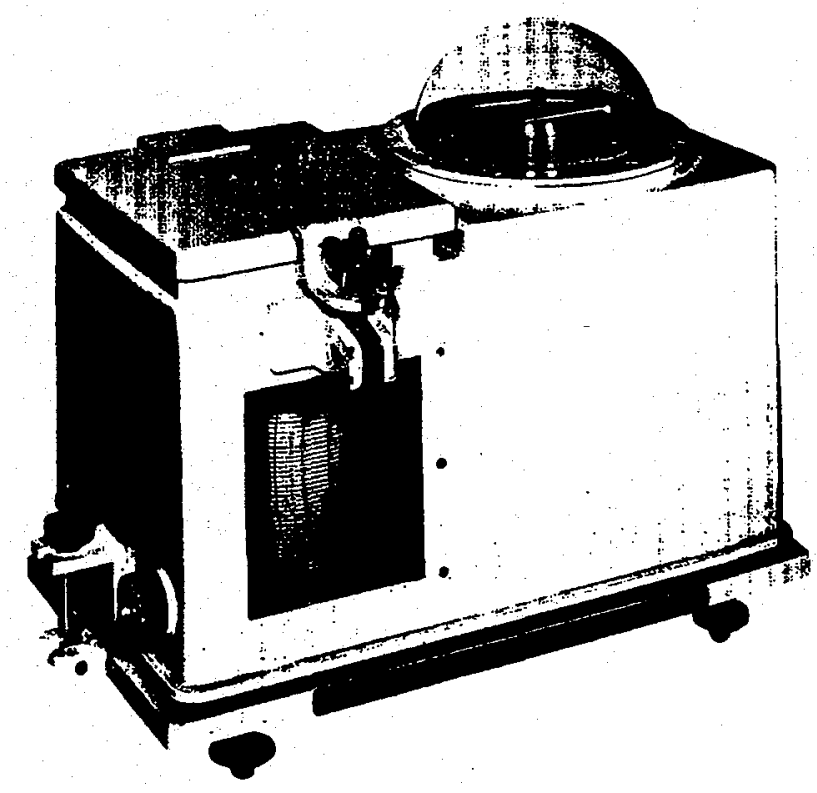

Bimetallic actinometer

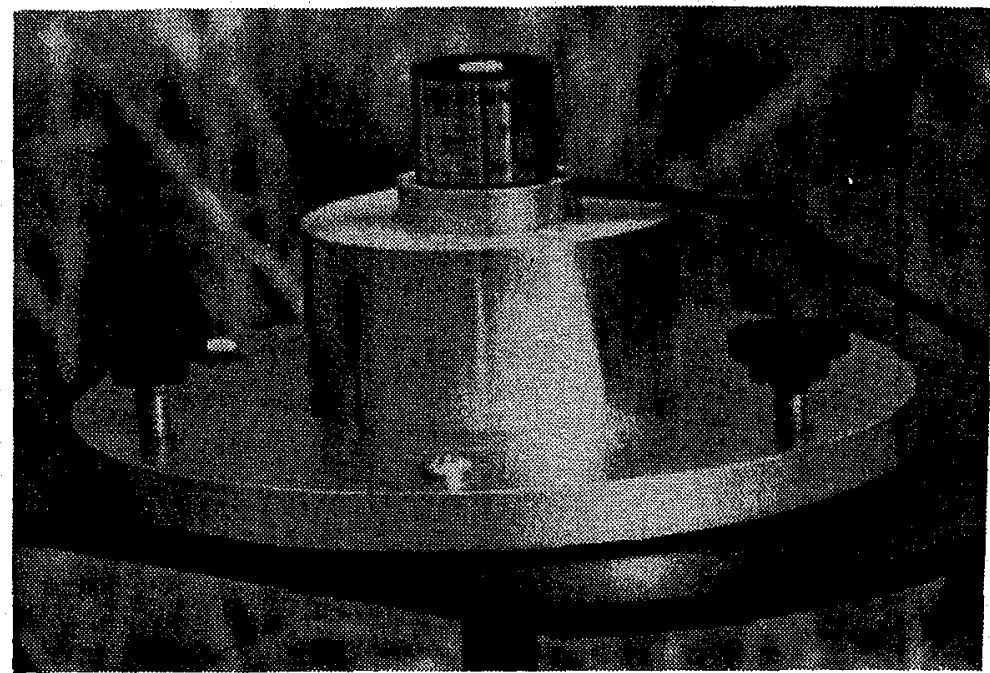

Photoelectric pyranometer

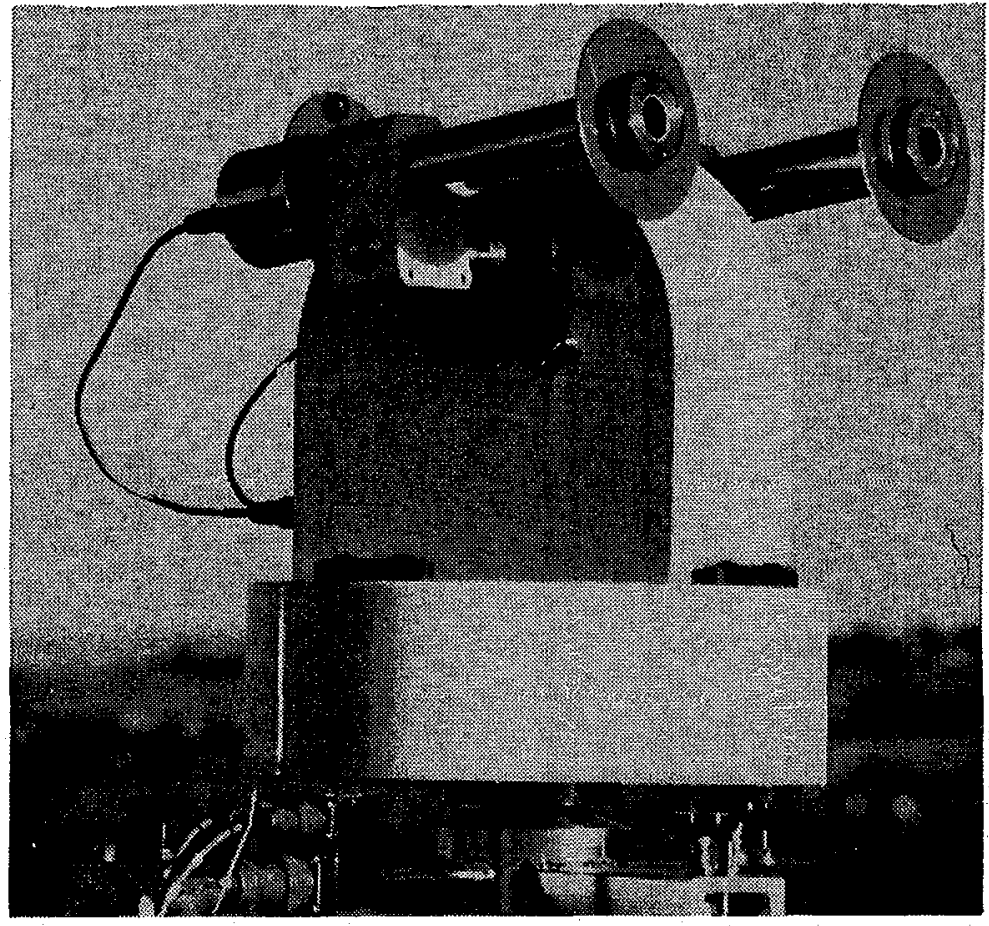

Thermopile pyrhellometer on a solar tracker

Fiaure 2-1. Examoles of solar radiometers 
recognize, however, that all historical and most current global horizontal data contain cosine errors. Depending on the design and construction of the instrument, the azimuthal position of the sun can also affect response to incident radiation.

Other errors that have particularly affected historical data, and are still of concern for most instruments today, include the effects of ambient temperature, spectral (color) variations in the response of the instruments (particularly serious with respect to photovoltaic devices), and nonlinear response to different intensities of solar radiation. For historical data the combination of these errors could exceed 10\% (SOLMET Vol. 2 1979).

Another problem of special significance for historical solar radiation data is the change in the response characteristics of the instruments with age or exposure to solar radiation. In particular, some of the instruments in use from the early 1950 s through 1975 were subject to significant reductions in sensitivity due to changes in the color of the paint used to coat the absorbing surface (Flowers and Starke 1967). Instruments using Parson's Black paint were observed to change to gray and then to green after several years of exposure in regions with high levels of solar radiation. These color changes resulted in decreases in sensitivity as great as $15 \%$ (Flowers and Starke 1967).

As if these characteristics of the devices used to measure solar radiation did not present enough problems, we have also been faced with major uncertainties in their calibration. In the field of metrology, one generally has standards that are used to calibrate measurement instruments. Voltmeters can be calibrated against standard cells (batteries), thermometers can be adjusted using the triple-point-ofwater (that temperature when the solid, liquid, and vapor states are in equilibrium), and our clocks can be calibrated against a cesium clock, which is regulated by the atomic absorption characteristics of cesium atoms.

Unfortunately, the standard sources of radiation that can be used to calibrate solar radiometers have significant deficiencies. There is no artificial source of radiation that can accurately simulate the spectral characteristics of radiation from the sun and provide a beam or distributed source of radiation of known intensity that is stable and capable of uniformly irradiating the radiometer.

In the absence of a standard radiation source, a new measurement scale for solar radiation was established in the years from 1970 to 1975 . That scale, called the World Radiometric Reference (WRR), was based on the responses of some carefully constructed and characterized pyrheliometers called absolute cavity radiometers. This measurement scale is maintained with a total uncertainty not greater than $\pm 0.3 \%$ (Romero, Fox, and Fröhlich 1990), using a group of seven selected absolute cavity radiometers forming the World Reference Group, operated by the World Radiation Center in Davos, Switzerland (SMI 1991). 
Absolute cavity radiometers (pyrheliometers) compare the heating effect of solar power with that of electric power. The direct beam of radiation coming from the solar disc passes through an aperture of accurately known area and is absorbed in a black cavity, which heats the cavity a small amount. Then, with the aperture covered an electrical heater is used to heat the cavity the same amount, and the electrical power to that heater is measured. This substitution measurement (electrical power for solar power) permits the power to be computed in absolute units of watts per square meter. Thereby, solar power (irradiance) can be related to national and intemational measurement standards of length (for the diameter of the precision circular aperture) and electrical units for power (voltage and resistance), and thus the term "absolute radiometer" can be used.

Commercial absolute cavity radiometers are generally rated by their manufacturers to have accuracies of about $\pm 0.5 \%$. But to confirm that accuracy and establish measurement traceability to WRR, the commercial radiometers must be compared to absolute cavity radiometers that participated in the International Pyrheliometer Comparisons (IPCs) sponsored by the World Meteorological Organization (WMO). The IPCs are held at the World Radiation Center in Davos, Switzerland, every five years $(1975,1980,1985,1990$, etc.), where comparisons are made to the World Standard Group used to maintain WRR (SMI 1991). The cavity radiometers are then used to calibrate field pyrheliometers and pyranometers to varying accuracies that depend upon the particular procedures and techniques used.

This already complex process is further complicated by the need to measure the different components of solar radiation incident on the surface of the earth. As noted earlier, a pyrheliometer is used to measure the direct beam radiation coming from the solar disc. Part of the radiation from the sun, however, is scattered by gases and aerosols in the earth's atmosphere and by clouds. The intensity of the scattered radiation (the diffuse component) is a function of the turbidity of the atmosphere and the amount and type of cloud cover. The direct beam and diffuse components of solar radiation combine to form the global or total solar radiation incident on a horizontal surface.

For many applications, one simply needs to know the global horizontal radiation from the sun. For concentrating or focusing collectors, however, the direct beam component is the one of importance. And for many interior lighting applications, the diffuse component is of greatest interest. For tilted or tracking flat plate collectors, the direct and diffuse components are used to calculate the energy incident on the collector surface. The measurement of each of these three fundamental components presents its own set of problems.

Routine measurement of the direct beam component requires a tracker to continually point a pyrheliometer at the sun. Any errors in tracking the sun produce errors in the measurement of the direct beam of solar radiation. To measure the diffuse component, one has to shield a pyranometer from the direct beam component. If this shielding is accomplished with a tracking disc, which blocks only the direct beam from the solar disc, the measurement accuracy is 
determined only by the accuracy of the pyranometer itself. Tracking discs are expensive, however, and are subject to the same tracking errors as these noted for the measurement of the direct beam component. To avoid these tracking problems and the expense of a solar tracker, the measurement of the diffuse component is more frequently accomplished using a shadowband, which is adjusted every few days to block the direct beam component during the passage of the sun across the sky. Figure 2-2 shows both instruments used to measure diffuse solar radiation.

If the shadowband is adjusted every few days, one should not encounter errors due to failure to block the direct beam. However, the shadowband not only blocks the direct beam component, it also blocks a certain portion of the diffuse radiation. If the radiance from the sky were homogeneous and isotropic, a precise correction could be made for that portion of the diffuse radiation that is blocked by the shadowband. However, the radiance distribution within the celestial dome is not homogeneous and isotropic. It is a function of the location of the sun; the turbidity of the atmosphere; and the number, type, and location of clouds. Therefore, the correction for the shadowband is always an approximation that introduces a random source of error in the measurement of the diffuse component.

The measurement of global horizontal radiation is accomplished with a pyranometer positioned in the horizontal plane and is the easiest of all of the three components to measure. It is subject, of course, to all of the measurement errors associated with the characteristics of these instruments, as previously discussed. In actuality, however, even this measurement is not as simple as it might seem. In the first place, the receiving surface of the sensor must be precisely positioned in the horizontal plane. Although these instruments contain spirit levels that are quite accurate, it has been shown that the spirit level and the receiving surface of the sensor are sometimes misaligned, resulting in an improper leveling of the surface of the sensor. Furthermore, the pyranometer must be placed in a position so surrounding objects do not occlude the direct beam from the sun at any time during the day, and so the pyranometer is not exposed to scattered radiation from nearby objects. In many measurement locations, it is very difficult to meet both of these criteria.

Finally, we must consider the human factor and the errors this can produce. Even if perfect instruments were available, if they are improperly installed or improperly maintained, the measurement of solar radiation will not be as accurate as one would desire. Furthermore, all of the field instruments contain domes, windows, or diffusers through which the solar radiation must pass before it reaches the sensor. It is necessary, therefore, to keep them free of dirt, water, frost, snow, etc. to obtain an accurate measurement of the incident solar radiation. Any failure of the operator of a measurement station to maintain the instruments can result in errors. However, even with the most dedicated operator, there are likely to be periods of time when trackers have failed, when snow or other materials have been deposited on the domes, or when other troubles arise and the operator is not present to make the corrections until several hours after the problem occurred. 


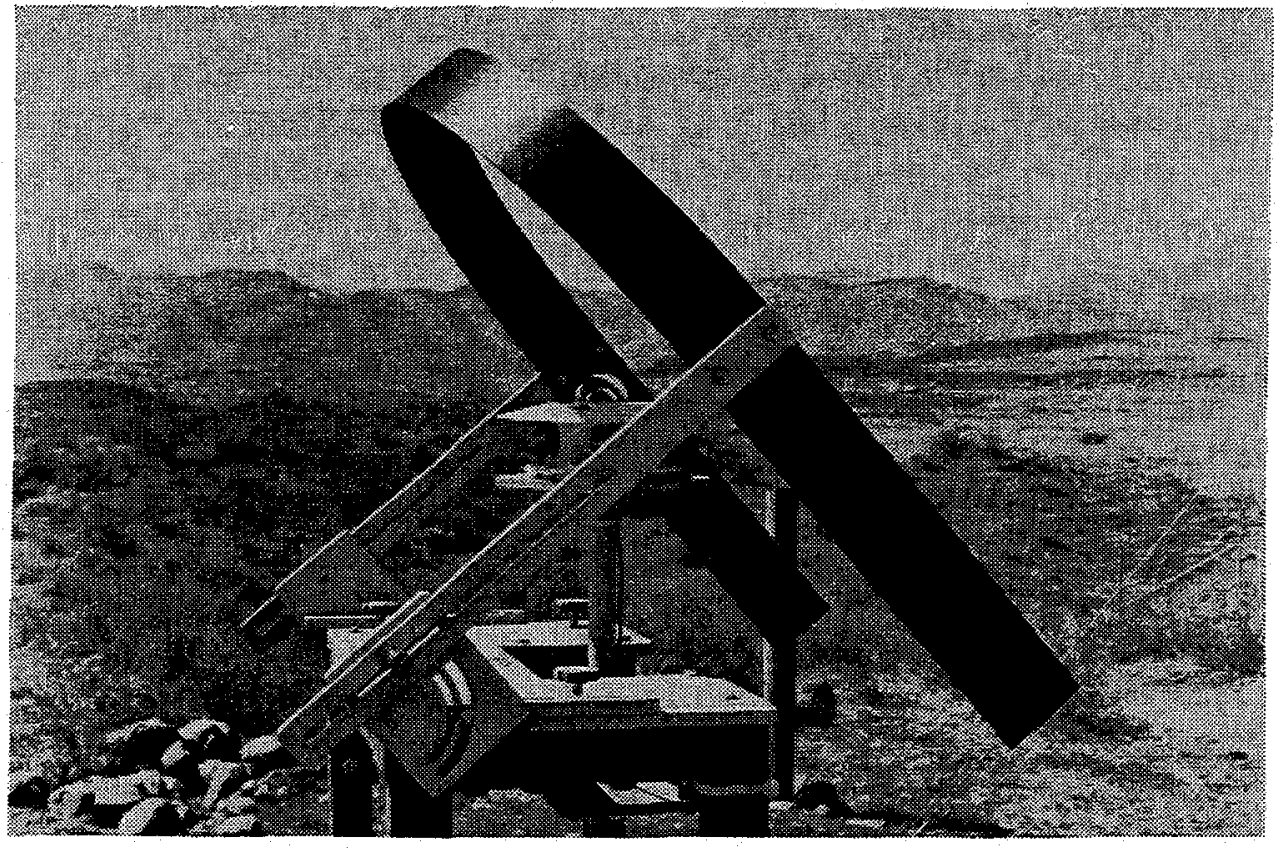

Shadowband diffuse radiometer

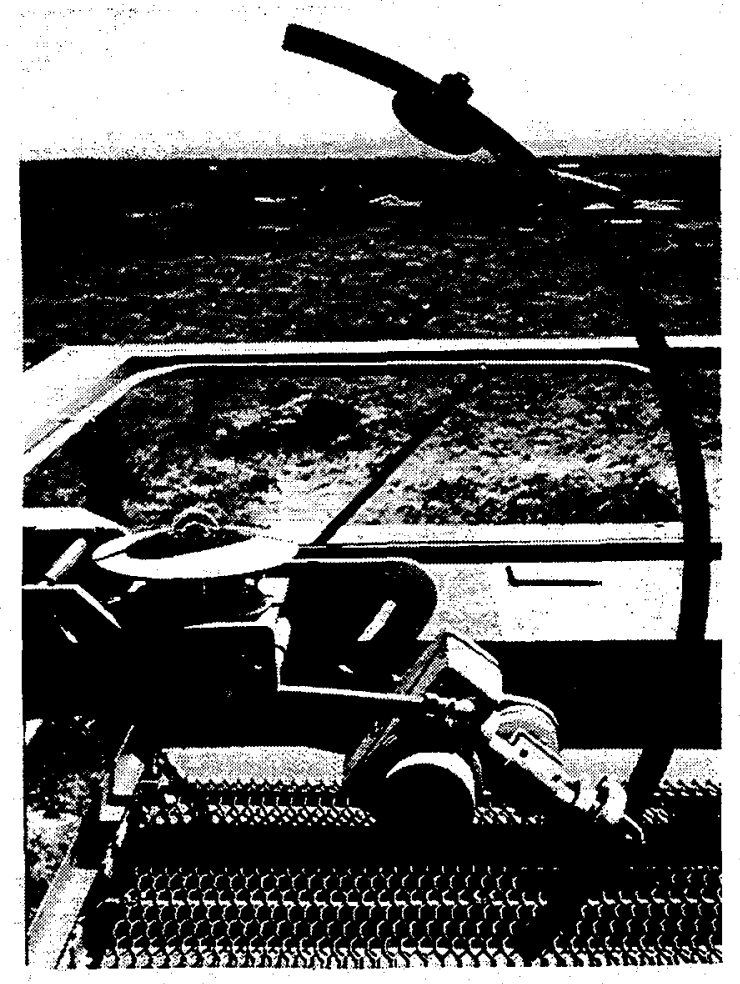

Tracking disc diffuse radiometer

Figure 2-2. Instruments used to measure diffuse solar radiation 


\section{References}

Flowers, E.C. and Starke, P.P. (1967). Results of a Field Trip to Compare Pyranometers. A NOAA report that was never published. Copies are available from NREL, Golden, CO.

Romero, J., Fox, N.P. and C. Fröhlich. (1990). "First Comparison of the Solar and an SI Radiometric Scale." Presented at the New Developments and Applications in Optical Radiometry III, Davos, Switzerland, 20-22 September 1990. Published in Metrologia, Vol. 28, No. 3 (September 1991); pp. 125-128.

SMI. (1991). International Pyrheliometer Comparisons - IPC VII; 24 September to 12 October 1990 - Results and Symposium; Working Report No. 162, Swiss. Meteorological Institute; Davos and Ceric, March.

SOLMET Vol. 2. (1979). Final Report - Hourly Solar Radiation - Surface Meteorological Observations, TD-9724, Asheville, NC: National Climatic Data Center. 



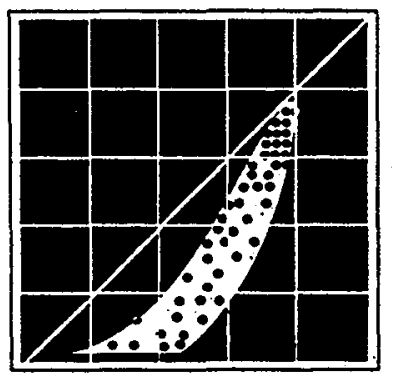

\section{Chapter 3 Quality Control Measures and Options}

The control of the quality of most measurements is relegated to the control of the measuring instruments and measuring processes themselves. An accurate measurement will usually result from the use of a high-quality instrument that has been accurately calibrated and is properly used by a qualified individual.

These criteria can also be applied to the measurement of solar radiation when the instrument has been under the constant control of a qualified individual during any given measurement. Such control can be maintained in connection with the performance evaluation of a solar collector. But for routine continuous monitoring of solar radiation in a network containing many stations and several radiometers at each station, constant control of the measurement process is impossible. At most stations, it is likely that the radiometers are examined at most once or twice each day. Therefore, even under the best of circumstances there will be opportunities for significant errors to be made for a finite percentage of the measurements in a given year. Under the worst of circumstances, with a poorly calibrated instrument that is improperly installed and improperly maintained, all of the measurements taken can be in error by a significant amount. Of course, everything should be done to control as many of the factors related to accurate measurement as possible. This will result in good quality and accuracy for most of the measurements.

Given the differences in training, experience, and dedication of individual station operators, the uncontrollable circumstances connected with the operation of a station, and the problems with solar radiometry in general, it becomes unreasonable to assume that all of the measurements made in a national network will be of the quality and accuracy desired. Under these circumstances, one must undertake a postmeasurement quality assessment of the data collected.

Postmeasurement quality control or quality assessment has been a part of most network operations. For the most part, however, these quality assessment measures have been limited to comparisons with physical limits, which identify data that are grossly in error due to the failure of the instruments or station maintenance. Most commonly, measured solar radiation data have been judged to be acceptable when the irradiance level is greater than zero and less than that which would be expected outside the earth's atmosphere, at the time and under the circumstances of the actual measurement. In other words, postmeasurement quality assessment has most frequently been limited to comparisons with zero and extraterrestrial 
radiation (ETR). This obviously does not meet our criteria for setting ranges of expected values to produce a small percentage of Type 1 errors.

Comparison of measurements with model estimates could also be used for quality control. Under cloudless skies and with accurate information regarding the precipitable water vapor and turbidity of the atmosphere, the current solar radiation models can very accurately estimate the direct beam component of solar radiation. Even the diffuse component can be estimated with reasonable accuracy under these conditions. If a measured value of solar radiation is then compared with model estimates, this provides a better quality assessment than do comparisons with zero and ETR. The major shortcoming of this approach to quality assessment is the lack of accurate input data to the models.

Precipitable water vapor measurements using radiosondes are usually made only twice each day and turbidity measurements are made at only a few locations around the United States. Turbidity measurements are infrequently made at other locations in the world. In the final analysis, one quickly determines that the measurement of precipitable water vapor and turbidity are more manpower intensive and are more expensive and difficult than the measurement of solar radiation itself. Therefore, even for cloudless sky conditions, the use of models for quality assessment is not a good solution. When clouds are present, the use of models for quality assessment is not at all practical, because models can only estimate monthly means and the statistical variability of solar radiation under given cloud conditions.

It is obvious that quality assessment of solar radiation data presents a serious dilemma. There are many reasons to expect that measured values will contain significant errors from time to time. Using ETR and zero limits for comparison with measured values will only detect gross errors, resulting from human or equipment failures. The use of models for comparisons with measured values is impractical because the data inputs for the models are more difficult to obtain than are the solar radiation data themselves, and the use of redundancy (using two instruments to measure the same parameter) is rarely done because of the cost.

What other options are available for quality assessment of solar radiation data? All of the quality assessment options that we have been addressing call for the comparison of an actual measurement with an expected value of solar radiation. In the first instance, one is expecting that the solar radiation will lie between values of zero and ETR. As long as the measured data meet that expectation, they are declared to be acceptable. When using models to perform quality assessment, the model calculates the expected value of solar radiation under the given atmospheric conditions for the time and location where the actual measurement was made. The measurement is then compared with the modeled value and if the agreement between the two is within some defined limits, the measured data are deemed to be acceptable. Because of the problems associated with models and the very broad acceptance range associated with the physical limits of zero and ETR, some other 
method of arriving at expected values of solar radiation is needed to improve the quality assessment of these data.

After much consideration, it was determined that solar radiation data themselves will provide the best estimate of the values to be expected at any given location and month for any given hour. At first this might seem to represent a paradox, to suggest that the measured data should be used to establish the expected values to be used for quality assessment. However, the following considerations support this approach:

- It is assumed that good quality, properly calibrated, properly installed, and properly maintained instruments have been used to collect the data. Therefore, erroneous data should be in the minority and should represent infrequent failures of the equipment or operator maintenance.

- If a model were used to establish expected values, one must remember that the model itself would have been developed and validated using the same data we propose to use to establish expected limits or quality control.

- $\quad$ The use of past data to establish expected values requires no meteorological data for input to a model. This is especially important for some measurement locations where meteorological data are not collected.

This empirical approach to quality assessment is at a disadvantage for assessing the data from a new station for which there is no historical record. However, based on our experience with this approach to quality assessment, it is highly probable that reasonably good estimates of expected values can be made by comparing the climate of the new location with other locations having similar climates. This would provide a starting point for the initial quality assessment during the first year of operation. Following that first year, some modification of expected values should be expected, based on the availability of more measured data.

This apparent disadvantage can also be viewed as an advantage, because this empirical approach to quality assessment is a dynamic one. In other words, even for a station with 20 years of historical data, climate changes can result in a change in the expected values of solar radiation. The user of this approach to quality assessment can employ the results of the assessment to detect changes that might represent long-term climate variations and/or the effects of natural or man-made events that change atmospheric conditions. For example, volcanic eruptions or man-made environmental pollution can change the turbidity of the atmosphere, thereby changing the expected values of solar radiation at the surface of the earth. If such changes are significant, the user of the quality assessment methodology described in this manual should detect a change in the assessment results (the frequency and type of quality flags should change) consistent with the change in climate or atmospheric conditions. 



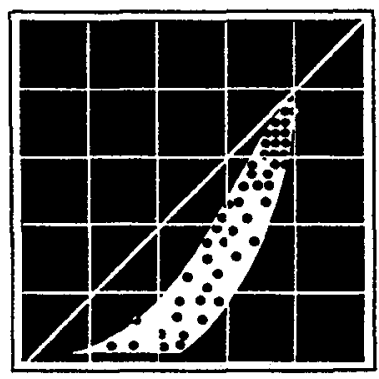

\section{Chapter 4}

\section{Conceptual Description of SERI QC}

SERI QC assesses the quality of solar radiation data by comparing measured values with expected values. The natural variations of solar radiation with time of day, latitude, and season make it difficult and confusing to define or discuss the expected absolute values of these parameters in the context of quality assessment. Communications are further complicated by the variety of units used to express solar radiation values, including Langleys, Btu/ $\mathrm{tt}^{2}, \mathrm{kWh} / \mathrm{m}^{2}$, and $\mathrm{mJ} / \mathrm{m}^{2}$ for energy values; and $\mathrm{W} / \mathrm{m}^{2}$ or $\mathrm{kW} / \mathrm{m}^{2}$ for irradiance values. Therefore, the rest of this manual and the SERI QC algorithm and software deal with unitless values that have been normalized with respect to extraterrestrial radiation (ETR). These parameters are defined according to the expressions

$$
\begin{gathered}
\mathrm{Kn}=\mathrm{I}_{\mathrm{n}} / \mathrm{I}_{\mathrm{o}} \\
\mathrm{Kt}=\mathrm{I}_{\mathrm{t}} /\left(\mathrm{I}_{\mathrm{o}} \cos \mathrm{z}\right) \text {, and } \\
\mathrm{Kd}=\mathrm{I}_{\mathrm{d}} /\left(\mathrm{I}_{\mathrm{o}} \cos \mathrm{z}\right) \text {, }
\end{gathered}
$$

where

$\mathrm{I}_{\mathrm{o}} \quad=$ extraterrestrial direct normal radiation

$I_{n} \quad=$ direct normal radiation at the earth's surface

I $=$ total global horizontal radiation at the earth's surface

$\mathrm{I}_{\mathrm{d}} \quad=$ diffuse horizontal radiation at the earth's surface

$I_{0} \cos z=$ extraterrestrial radiation on a surface parallel to the earth's surface

$\mathrm{Z}=$ solar zenith angle

$\mathrm{Kn}=$ direct beam ransmittance

$\mathrm{Kt}=$ cleamess index or effective global horizontal transmittance

Kd $\quad=$ effective diffuse horizontal transmittance.

The radiation units must be the same for each radiation parameter. 
The scatter plot of data shown in Figure 4-1 illustrates the advantages of working in K-space when using an empirical approach to quality assessment of solar radiation data. This plot includes all of the hourly data collected at Tallahassee, FL, from 1977 through 1980, for those hours when both global horizontal and direct nomnal data were collected and when the solar zenith angle was less than $80^{\circ}$. The two dashed lines shown in this figure establish the maximum expected values for $\mathrm{Kt}$ and $\mathrm{Kn}$ for the atmospheric conditions existing at Tallahassee, FL, during these four years. If we assume that these four years are representative of average conditions at Tallahassee, then these dashed lines provide quality assessment boundaries that can be used for any measurement of global horizontal or direct normal solar radiation at this location. This represents almost a 50\% reduction in the area of acceptability as compared to ETR and zero. In K-space, ETR equals 1.0.

When concurrent measurements are made of both global horizontal and direct normal solar radiation, the solid line encompassing the bulk of the measured values can be used to further reduce the range of expected values. The area of acceptability now is less than $14 \%$ of the total area between zero and ETR. When scatter plots such as this are made for each month of the year and for three air mass ranges, the area of acceptability is further reduced.

For example, Figure 4-2 is a similar scatter plot for Tallahassee for the month of July, limited to air mass values in the range from 1.25 to 2.5. The area of acceptability has now been reduced to less than $8 \%$ of the total area between zero and ETR. For higher air mass values (2.5 to 5.8) the plot in Figure 4-3 indicates an area of acceptability of about $5 \%$ of the total. Even if only one of the solar radiation components has been measured, the upper limit of expected values for $\mathrm{Kn}$ could be reduced to 0.3 ; and for $\mathrm{Kt}$, a value of 0.6 can be used. Notice that all of these boundaries have been set to leave a few data outside the region of acceptability. If all of the data are valid, these would represent Type 1 flagging errors (flagging good data as bad).

If all three of the fundamental components of solar radiation have been measured, the expected $\mathrm{K}$-value of any one of the three can be computed from the other two using the relationship

$$
\mathrm{Kt}=\mathrm{Kn}+\mathrm{Kd}
$$

It is possible, of course, that all three components could be in error by just the right amounts and still satisfy equation 4-4. However, the probability of this happening is quite low, because the sources of error for these three measurements (see Chapter 2, page 7) are different and independent.

If we consider a point in three-dimensional K-space to represent an accurate measurement of solar radiation, then the use of equation 4-4 to perform quality assessment is by far the best, because the region of acceptability can now be represented by a sphere, with a radius set to any range of acceptability desired. 


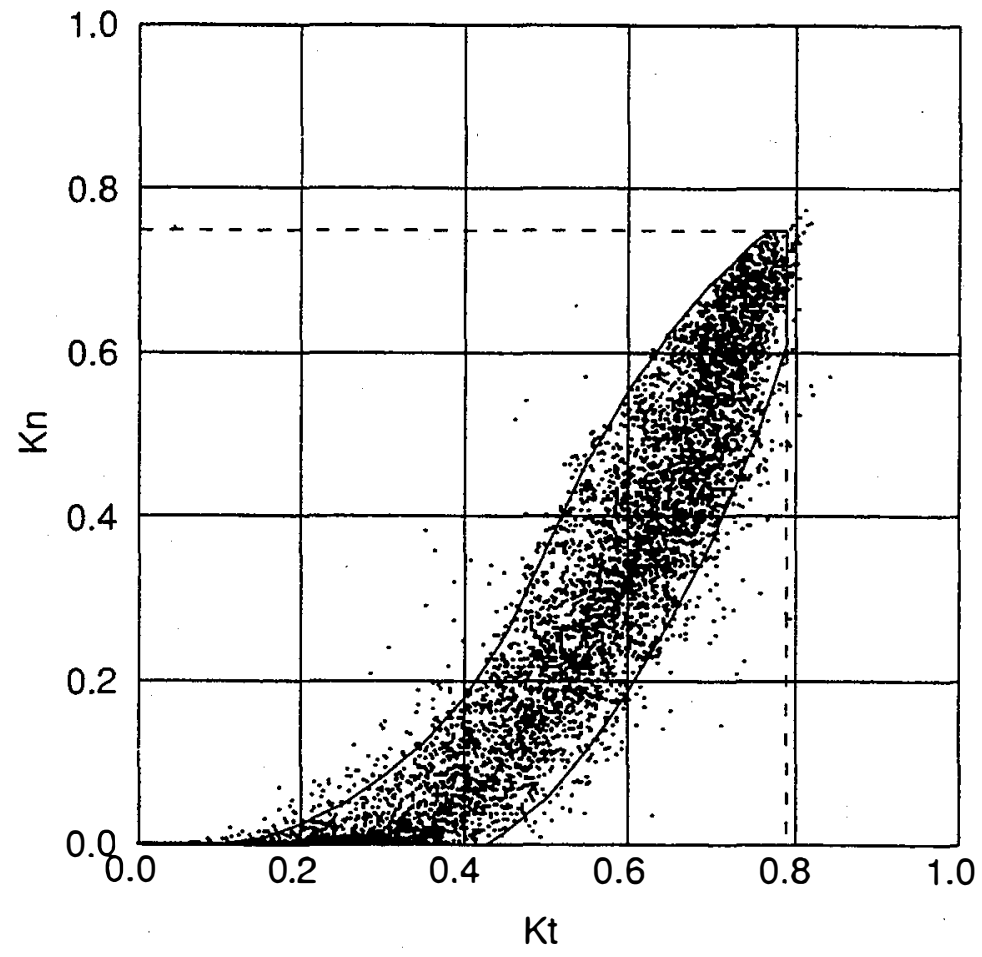

Figure 4-1. K-space scatter plot of all solar radiation data collected at Tallahassee, FL, from 1977 through 1980

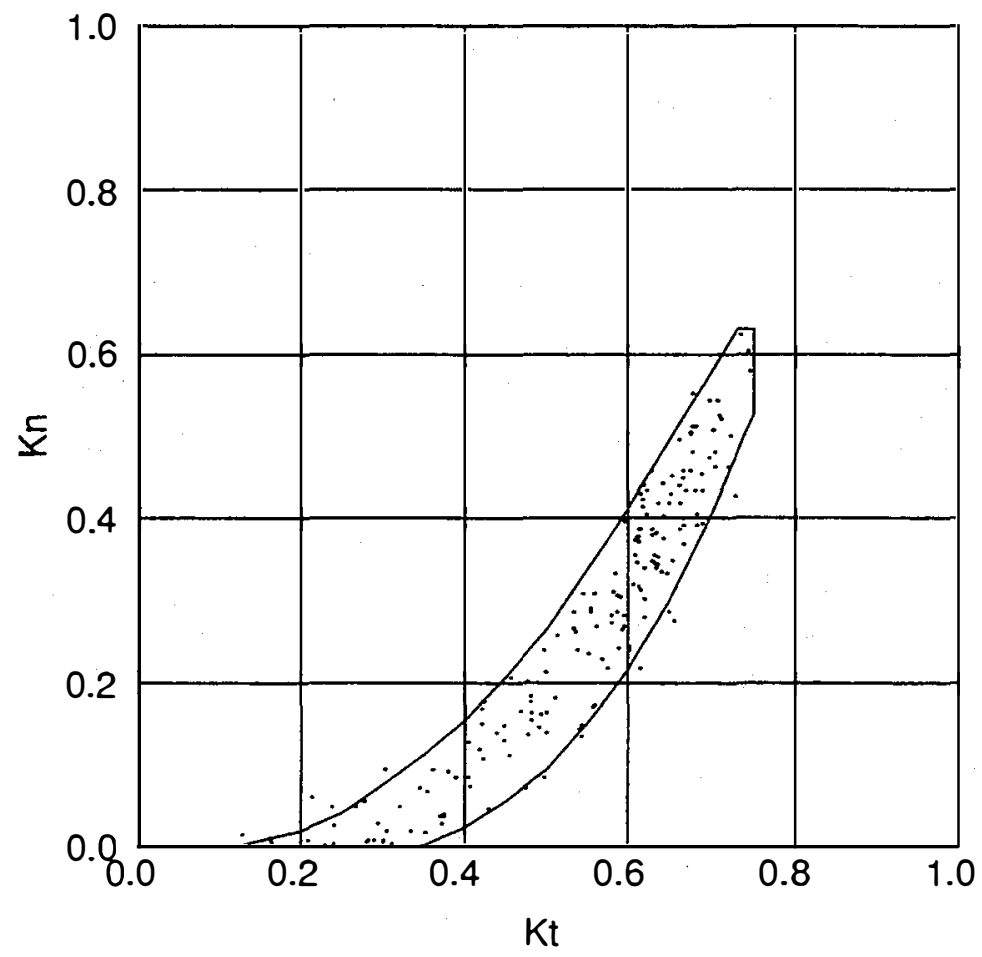

Figure 4-2. K-space scatter plot of medium air mass (1.25 - 2.5) solar radiation data collected at Tallahassee, FL, from 1977 through 1980 


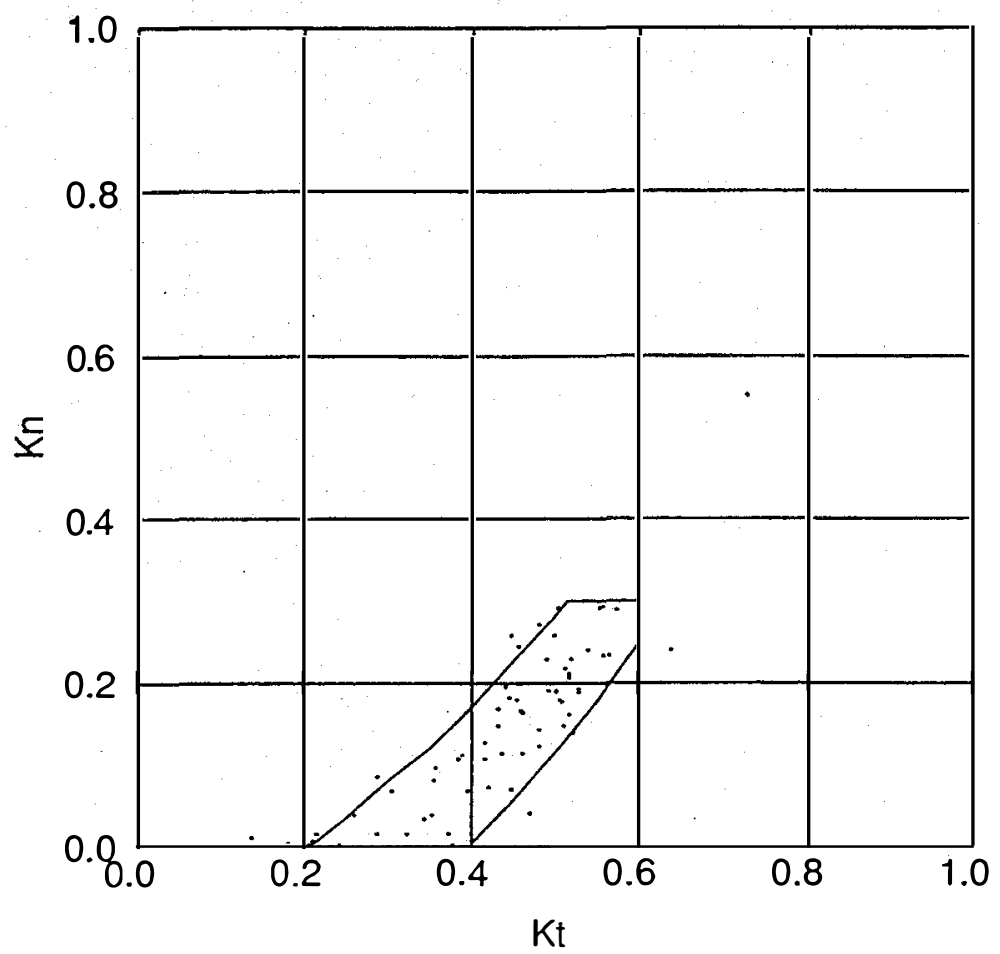

Figure 4-3. K-space scatter plot of high air mass (2.5 - 5.8) solar radiation data collected at Tallahassee, FL, from 1977 through 1980

\subsection{Theoretical Limits}

Several other lines have been added to the scatter plot of Tallahassee data and are plotted on Figure 4-4 to assist in the interpretation of K-space and the results of quality assessments using the procedures described in this manual. The diagonal line extending from 0,0 to 1,1 divides $\mathrm{K}$-space into theoretically possible and impossible regions. It is theoretically impossible for data to exist above this diagonal, because this would mean $\mathrm{Kt}<\mathrm{Kn}$ and $\mathrm{Kt}-\mathrm{Kn} \neq \mathrm{Kd}$ (because $\mathrm{Kd}$ cannot be negative).

The S-shaped curve passing through the right side of the data was generated by the Bird cloudless sky model (Bird and Hulstrom 1981). The upper part of the curve (above the dashed line) was generated using an air mass of 1.0 and a wide range of expected atmospheric conditions. The uppermost point represents an atmosphere with no water vapor and no aerosols. We have called this a Rayleigh atmosphere because the only scattering process is Rayleigh scattering produced by molecules of the uniformly mixed gases. 
The values between the Rayleigh point and the midpoint on the curve were generated by allowing water vapor and broadband turbidity to increase together in a manner consistent with the general correlation between these variables. The specific values used for water vapor and turbidity, respectively, are 0.0 and $0.0 ; 0.4$ and $0.01 ; 1.0$ and $0.05 ; 2.0$ and $0.10 ; 3.0$ and $0.20 ; 4.0$ and $0.33 ; 5.0$ and 0.50 ; and 6.0 and 0.7 , where water vapor is given in centimeters. From Ho and Riedel (1979) we find that maximum precipitable water values often approach or exceed $6.0 \mathrm{~cm}$ at coastal and even some inland locations; e.g., Ft. Worth, TX.

The bottom part of the curve was generated by fixing water vapor and turbidity values at 6.0 and 0.7 and by increasing air mass through a range of values from 1.0 to 6.0. A visual comparison of Figures 4-1 and 4-4 reveals little difference between the right boundary of Figure 4-1 and the calculated curve on Figure 4-4. This serves as a partial validation of the use of empirical boundaries for performing quality assessment.

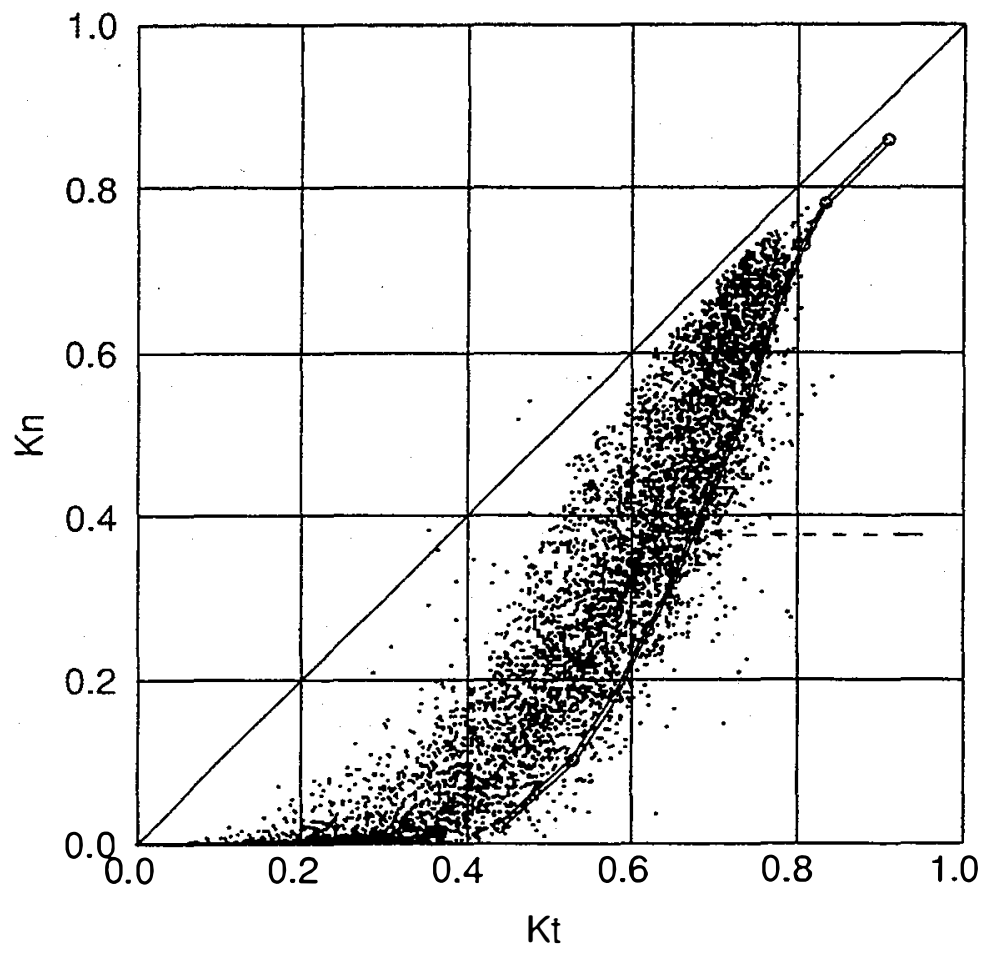

Figure 4-4. K-space scatter plot of all solar radiation data collected at Tallahassee, FL, from 1977 through 1980, with added interpretive information 


\subsection{Empirical Limits}

Confidence in a quality assessment procedure based upon empirical limits has grown primarily from the use of SERI QC to examine many years of data from over 40 stations for all 12 months of the year. The effects of air mass, climate, seasonal changes, and atmospheric parameters have been observed. A few examples of these effects are reproduced here.

\subsubsection{The Effects of Clouds}

As cloud cover increases, the direct normal component is observed to decrease more rapidly than the global component, because the clouds tend to increase the diffuse component while decreasing the direct beam component. This results in the left-hand boundary of scatter plots pulling farther and farther away from the 0,0 to 1,1 diagonal as the cloud cover increases and $\mathrm{Kn}$ and $\mathrm{Kt}$ decrease. This is clearly seen in Figures 4-1 and 4-4. Only under near-overcast conditions, as Kn approaches zero, do we see the data swing back toward the diagonal. This return to the diagonal is the result of the darkening of the clouds and the resultant decrease in the diffuse component of solar radiation under overcast conditions.

In addition to the normal processes of attenuation of the direct beam and spatially uniform increases in the diffuse component, clouds can produce unusual or phenomenal local increases in the diffuse component. For example, if the sun was to the west of the measurement location and there was a large cumulus cloud to the east of the measurement location, it is possible that the cumulus cloud would brightly reflect the incident solar radiation back toward the measurement location, resulting in a large increase in the diffuse component. Furthermore, it is frequently observed that when the sun is close to the edge of a cloud, there is an increase in forward scattering shortly before the cloud begins to occlude the direct beam. This is often referred to as focusing. Hulstrom (1973) notes that clouds can cause bright spots on the earth's surface, the result of as much as a $30 \%$ higher incident solar radiation than surrounding areas.

High values of diffuse radiation also are known to occur when snow is on the ground and clouds are in the atmosphere. These high values of diffuse radiation are the result of multiple scattering between the ground and the clouds. Because snow typically has an albedo of $0.6-0.8$, much of the solar radiation reaching the surface of the earth is reflected back into the atmosphere. If clouds are present, the energy reflected by the snow is reflected back to the surface by the clouds and so on. This can produce extremely high values of diffuse solar radiation, especially with extensive stratus cloud cover of thin to moderate thickness (not opaque). Under these conditions, it is possible for the global horizontal radiation to exceed ETR.

From results that will be shown later in this manual, we have learned that the enhanced diffuse radiation due to cloud phenomena is generally of short duration. 
This is evidenced by the increased frequency of occurrence of abnormally high Kt values as the period of measurement decreases from 1 hour to 1 minute.

Snow cover in Tallahassee is very unlikely. Hence, the data points lying to the right of the right-hand boundary in Figure 4-1 are most likely the result of cloud reflection and cloud focusing. Some of them could also be the result of imprecise tracking of the sun.

\subsubsection{Air Mass Effects}

The effects of air mass on both one-component and two-component limits and boundaries are shown in Figure 4-5. These results for Nashville, TN, in April are typical for the three air mass ranges used by SERI QC and defined in Table 4-1. Note that the curved boundaries move upward and to the left as air mass increases.

Also note that $\mathrm{Kt}_{\max }$ decreases by 0.03 and 0.07 as air mass increases from low to medium and medium to high ranges, respectively. Similarly, $\mathrm{Kn}_{\max }$ decreases in steps of 0.05 and 0.1 for these same increases in air mass. These incremental changes in $\mathrm{Kt}_{\max }$ and $\mathrm{Kn}_{\max }$ as air mass increases were found to be so prevalent that they were built into the SERI QC code. Larger changes are observed for very humid climates, but rarely do smaller changes occur.

Table 4-1. Air Mass/Zenith Angle Ranges

\begin{tabular}{|c|c|c|}
\hline Ranges & Air Mass & Zenith Angle \\
\hline \hline Low & $1.00-1.25$ & $0-36.96$ \\
\hline Medium & $1.25-2.50$ & $36.96-66.57$ \\
\hline High & $2.50-5.76$ & $66.57-80.00$ \\
\hline
\end{tabular}

\subsubsection{Atmospheric Effects}

Vertical movements in Kt-Kn space can be hypothetically attributed to the increasing turbidity or diffusiveness of the atmosphere. If atmospheric diffusion were increasing, with little or no increase in absorption or upward scattering, $\mathrm{Kn}$ would decrease and $\mathrm{Kt}$ would remain constant. Theoretically, if the atmosphere were to become a perfect diffuser, $\mathrm{Kn}$ would be near zero and $\mathrm{Kt}$ would be unchanged.

Movement along a line with a slope of 1.0 could be interpreted as the result of occluding the sun for a portion of the integration time. If the sun is completely occluded during the integration time, $\mathrm{Kn}$ will equal zero. To produce data along a line with a slope of 1.0, the occluding process cannot affect the diffuse component 

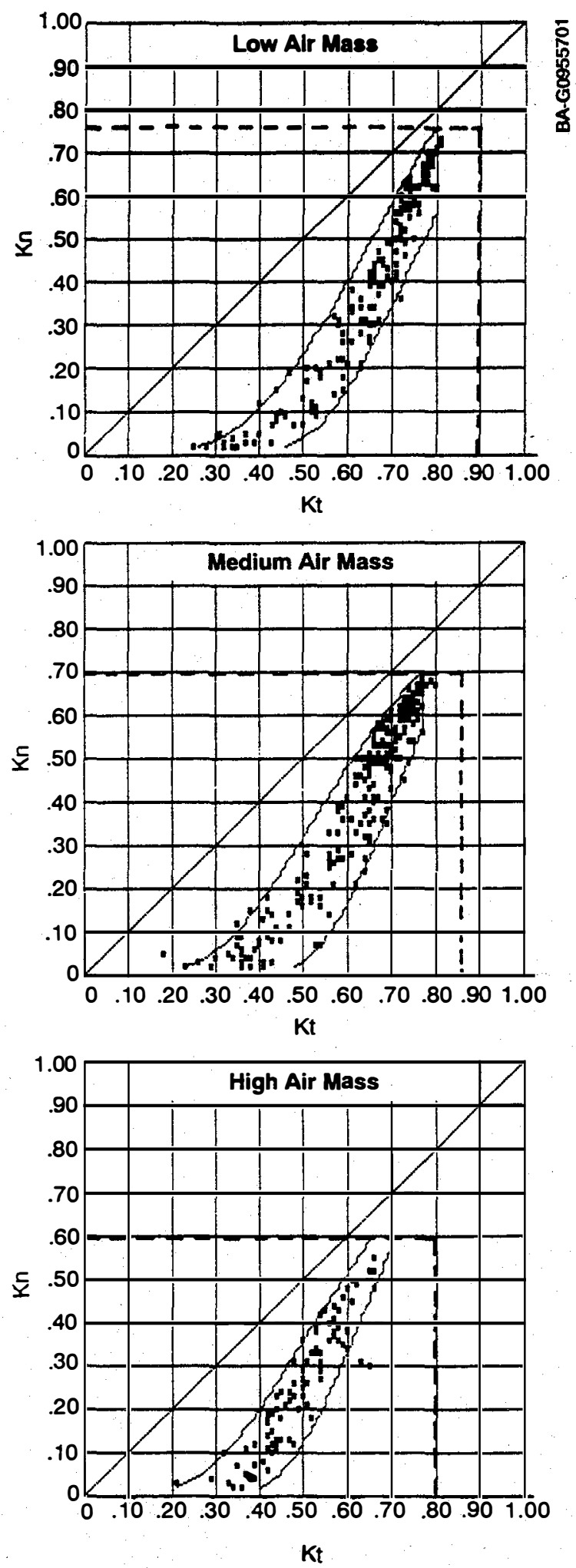

Figure 4-5. K-space scatter plots of solar radiation data for three air mass ranges for Nashville, TN, during April 
of solar radiation. This would be approximated during a solar eclipse, as the moon gradually occludes the direct beam.

From our examination of hundreds of station-months under a variety of atmospheric conditions, some general conclusions can be drawn about the actual atmospheric conditions that produce data points in the various regions of the Kt$\mathrm{Kn}$ space shown in Figure 4-6. The bulk of hourly data, usually over 90\%, lies within region C (see Figure 4-1). In general, the upper portion of region C corresponds to low cloud cover (clear) conditions, the middle portion to partly cloudy conditions, and the lower portion to cloudy conditions.

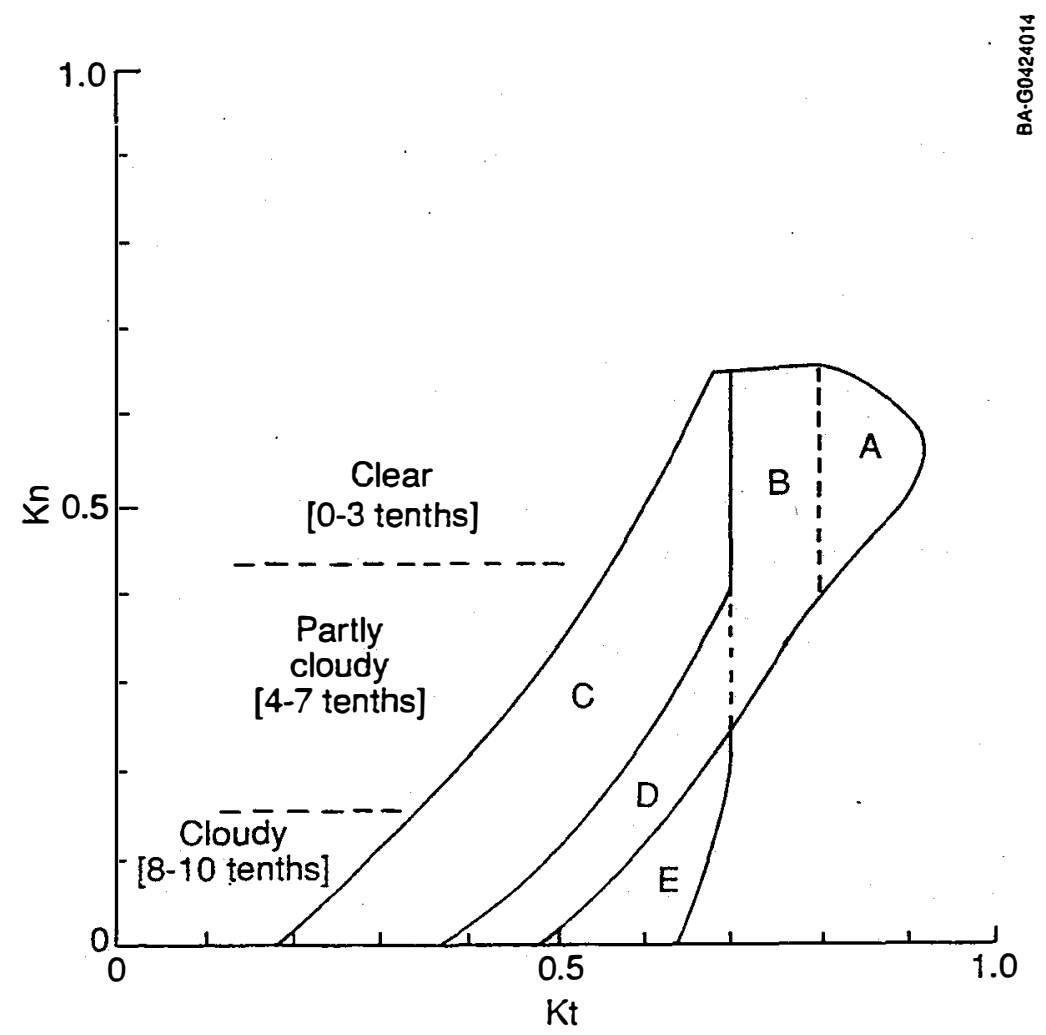

Figure 4-6. Regions in K-space that correspond to known atmospheric conditions 
Data within region A are observed primarily for short measurement times of less than 15 minutes. This indicates that the forward scattering or reflective phenomena from clouds that produce high diffuse values are relatively short lived. This is consistent with the fact that these data are also associated with relatively low cloud cover. Data within region $B$ are observed for integration times from 1 minute to 1 hour, with the frequency of occurrence decreasing as the integration time increases. Similar to region $\mathrm{A}$, these values must be produced by short-lived phenomena related to the scattering of light from nearby clouds. Data within regions $\mathrm{A}$ and $\mathrm{B}$ are observed most frequently for seasons and climates for which cumuloform clouds are frequently observed. The data within region $\mathrm{D}$, which are associated with medium to cloudy conditions, are seen for data averaged over short and long periods of time, although the frequency of occurrence decreases as the integration time increases. This again indicates that these are relatively short-lived phenomena. It should also be noted that the width of the flat region across the top of area $C$ is seen to decrease and often disappear as the measurement time increases from 1 minute to 60 minutes.

Good data falling within region $\mathrm{E}$ are most likely the result of a combination of cloud cover and high surface albedo. As noted in Section 4.2.4, this condition produces multiple scattering of light between the surface and the clouds, leading to very high diffuse values. As a consequence, if the clouds significantly reduce the direct beam component while increasing the diff use component, the data may fall in region $\mathrm{E}$.

The vertical line between region $B$ and region $C$ is clearly observed for hourly data when data points within region B are almost nonexistent (see Figure 4-5). This vertical boundary is apparently created by minimal atmospheric absorption and varying forward scattering. At the top of this vertical line we find data for very clear atmospheric conditions.

\subsubsection{Climate and Seasonal Effects}

As might be expected, the primary cause of climate and seasonal differences observed in the Kt-Kn plots of solar radiation data can be related to cloud cover changes. For example, the slope of the boundaries surrounding the data have been observed to decrease with the transition from winter cloud conditions to summer cloud conditions. This can also be associated with climate differences, as illustrated for Los Angeles, CA, and Lander, WY, in Figure 4-7.

From Hahn et al. (1984) we find that stratus and stratocumulus clouds are observed in the Los Angeles basin about $50 \%$ of the time during the summer. Cumulus and cumulonimbus clouds on the other hand are observed less than $10 \%$ of the time. Furthermore, from the narrative climatological summaries, published by NOAA in their Local Climatological Data Summaries, we learn that during the spring and summer months low cloud cover during the moming followed by sunny aftemoons is frequently observed. This, combined with the general high turbidity found in the Los Angeles basin, produces very steep boundaries. 


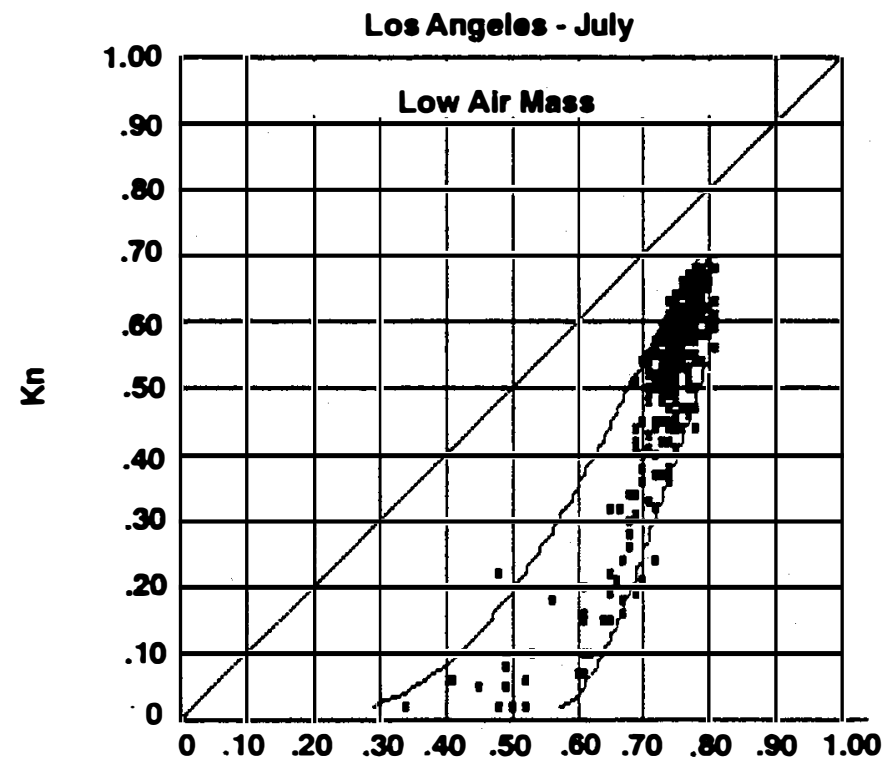

K!

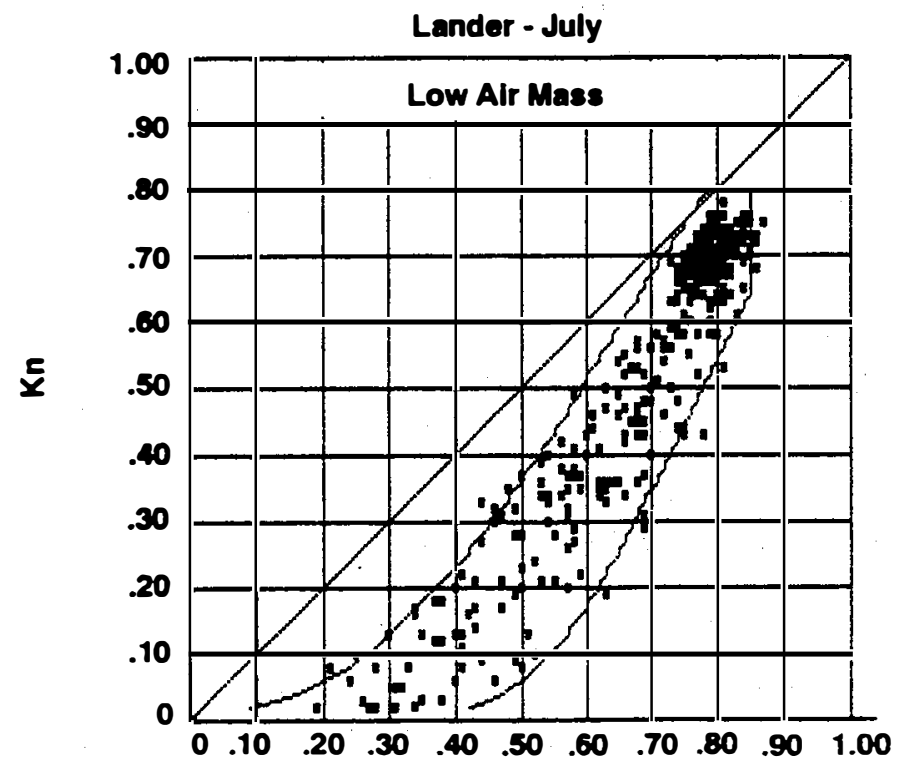

Kt

Figure 4-7. K-space plots for Los Angeles, CA, and Lander, WY, illustrating cloud-climate effect 
At Lander, WY, in July, cumulus and cumulonimbus clouds are observed approximately $50 \%$ of the time, whereas stratus and stratocumulus clouds are observed only about $15 \%$ of the time. Furthermore, we learn from the climatological summaries that clear and partly cloudy skies dominate the conditions at Lander during the summertime. With scattered cumulus clouds, there is the opportunity for the sun to be obscured for time periods ranging from a few minutes to over an hour, resulting in the wide scatter of data and boundaries with a lower slope. Contributing to this difference between Los Angeles and Lander is the relatively low turbidity generally observed in Wyoming, where the deer and the antelope still outnumber the automobiles (personal observation).

As was noted above, this reduction in slope also accompanies the transition from winter to summer at most locations in the United States. This is consistent with the dominance of cirrus and stratus cloud cover during the wintertime and the dominance of convective clouds (cumulus and cumulonimbus) in the summertime (Hahn et al. 1984).

Additional cloud climate effects can be noted by comparing plots of solar radiation data for Fresno, CA, in July; Montgomery, AL, in August; and Boise, ID, in March (see Figure 4-8). During July in Fresno, there are an average of 27 clear (0 to 3 tenths cloud cover) days and only 1 day with over 8 tenths cloud cover from sunrise to sunset (Local Climatological Data Summaries). This produces the results shown, where more than 9u\% of the data are clustered at very high $\mathrm{Kn}$ and $\mathrm{Kt}$ values. Furthermore, the separation between the right and left boundaries is very narrow, a further indication of the consistently clear skies that dominate the San Joaquin Valley during the summertime.

In contrast to these conditions at Fresno, wider boundaries are observed for Montgomery, AL, which typically experiences 8 clear days, 14 partly cloudy days and 9 cloudy days, during the month of August. This encompasses a broad mixture of cloud types. This feature, combined with high humidity (Local Climatological Data Summaries) produces the wide and uniform scatter of $\mathrm{Kt}$ and $\mathrm{Kn}$ values observed in this figure.

Finally, at more northerly, snowy climates high albedo effects are observed. For Boise, ID, in the month of March, we observe high Kt values even with $\mathrm{Kn}$ below 0.2 . This is very likely the result of multiple scattering between a snow-covered surface and clouds. Similar results are also observed for such locations as Bismarck, ND; Burlington, VT; and Caribou, ME. For these locations and months the right boundaries of the scatter plots become quite steep, approaching a vertical line. This is consistent with our hypothesis that movement along a vertical line in the Kt-Kn space represents a reduction in direct normal values with a concurrent increase in diffuse values. In other words, with a bright snow-covered surface and a diffusing, more or less uniform cloud cover, the global horizontal values of solar radiation remain very high. These are but a few of the seasonal and climate related conditions that one observes in the United States. 

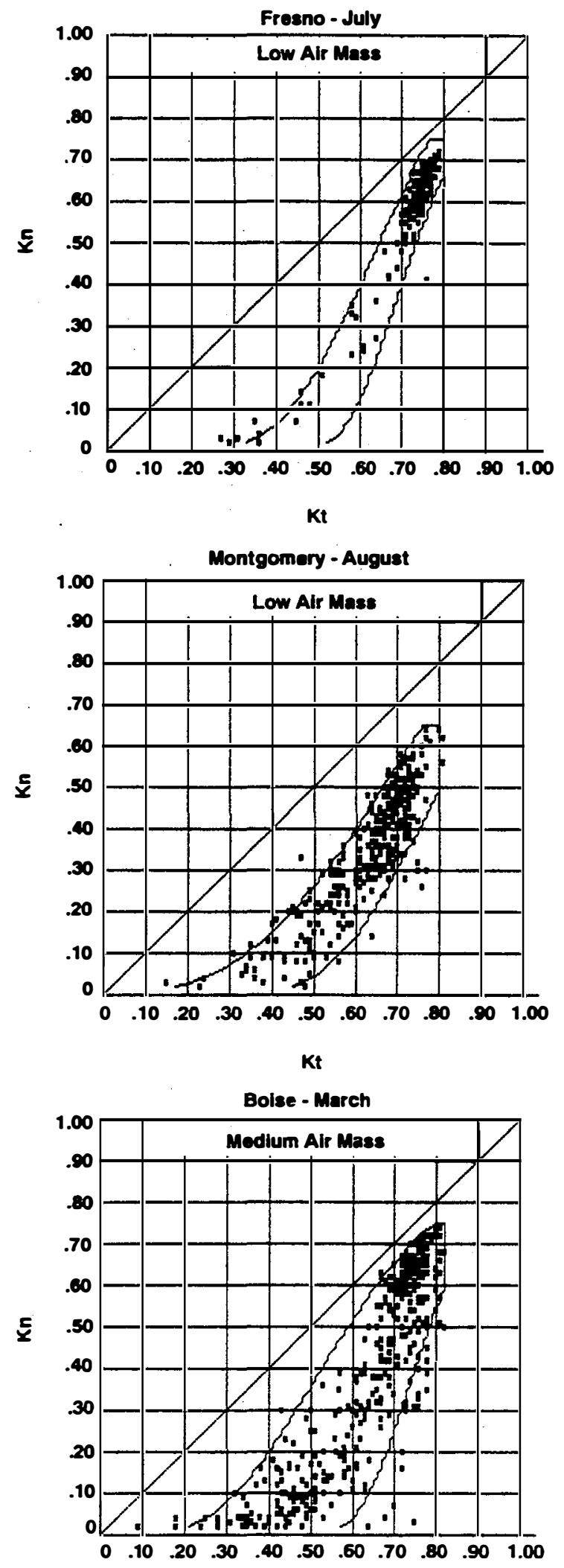

Kt

Figure 4-8. K-space plots for Fresno, CA, Montgomery, AL, and Boise, ID, illustrating cloud-climate effects 


\subsection{References}

Bird, R.E. and Hulstrom, R.L. (1981). A Simplified Clear Sky Model for Direct and Diffuse Insolation on Horizontal Surfaces, SERITR-642-761, Golden, CO: Solar Energy Research Institute.

Hahn, C.J., et. al. (1984). Atlas of Simultaneous Occurrence of Different Cloud Types Over Land. NCAR/TN-241+STR, Boulder, CO: National Center for Atmospheric Research.

Ho, F.P. and Riedel, J.T. (1979). Precipitable Water Over the United States - Vol. II: Semimonthly Maxima. NOAA/TR-NWS20, Silver Spring, MD: National Weather Service.

Hulstrom, R.L. (1973). “The Cloud Bright Spot," Jour. Photogrammetric Engineering, Vol. 39, pp. 370-376. 


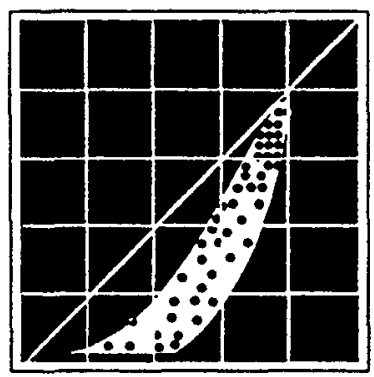

Chapter 5

\section{Quality Assessment Boundaries}

Once the decision was made to employ empirical boundaries to define the limits of acceptable data, we were faced with the problem of defining those boundaries. It was recognized quite early that the boundaries varied as a function of climate and season as described in Chapter 4, Section 4.2 (page 24). It also was evident that the quality assessment would be significantly compromised if the extreme left and right boundaries found for all station-month combinations were used for all stations. A search was undertaken, therefore, to find a function that would closely match the boundaries of actual data and that could be easily adapted to efficient software for performing the quality assessment function.

\subsection{The Gompertz Function}

The function that was finally selected is a double exponential called the Gompertz equation (Parton and Innis 1972), which for our purposes takes on the form

$$
\mathrm{Kn}=\mathrm{AB} \mathrm{CB}^{\mathrm{Dkt}}
$$

The effect of each of the four parameters in this equation makes it ideal for establishing boundaries for solar radiation data plotted in Kt-Kn space. As shown in Figure 5-1, the Gompertz function takes on shapes similar to the boundaries we have observed for scatter plots of solar radiation data in Kt-Kn space. Furthermore, the individual effects of the four coefficients provide the control needed to vary the shape and position of the boundaries. As illustrated in Figure 5-1, A determines the asymptotic value for $\mathrm{Kn}, \mathrm{B}$ positions the inflection point along the $\mathrm{Kn}$ axis, $\mathrm{C}$ positions the inflection point along the Kt axis, and D controls the slope of the curve at the inflection point. This function has proven to be invaluable for establishing quality control boundaries. 


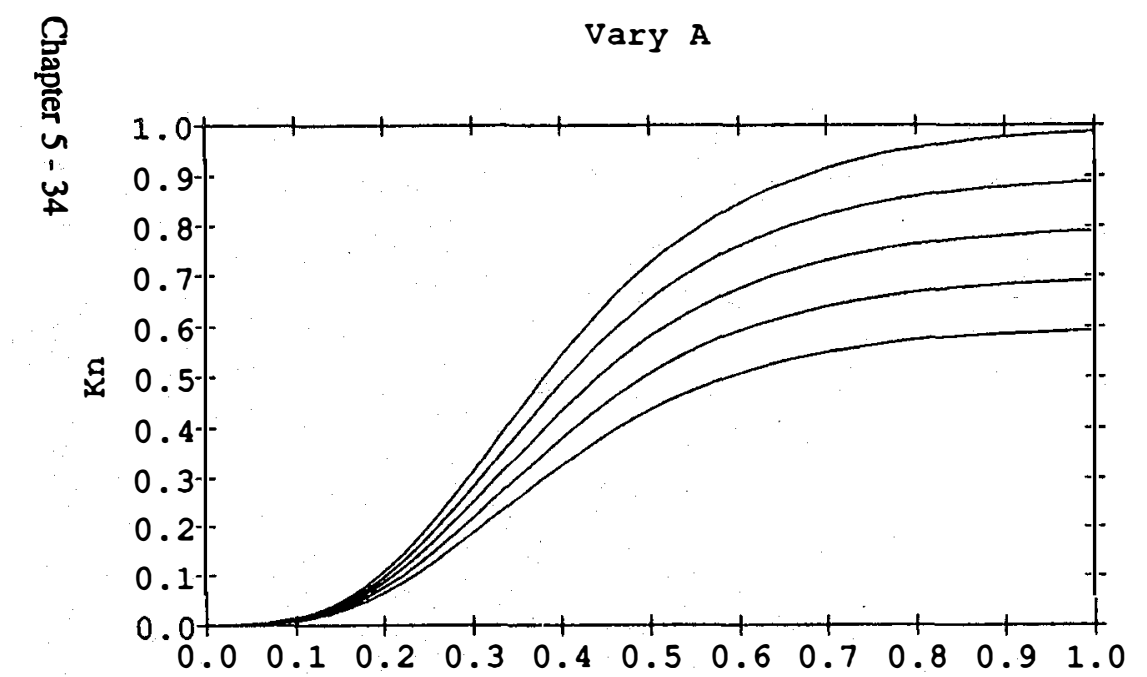

Kt

Vary $\mathrm{C}$

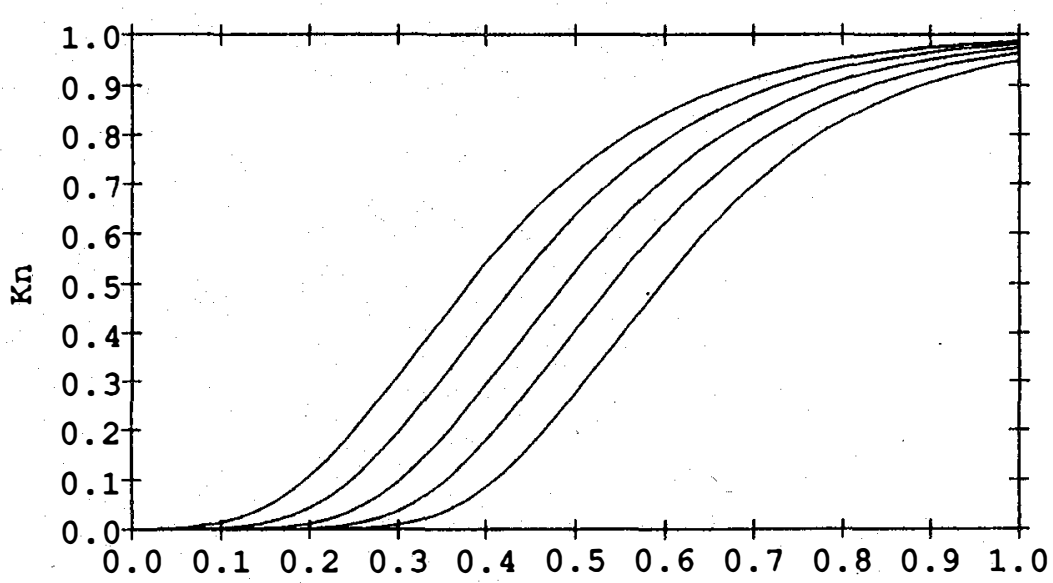

Kt
Vary B

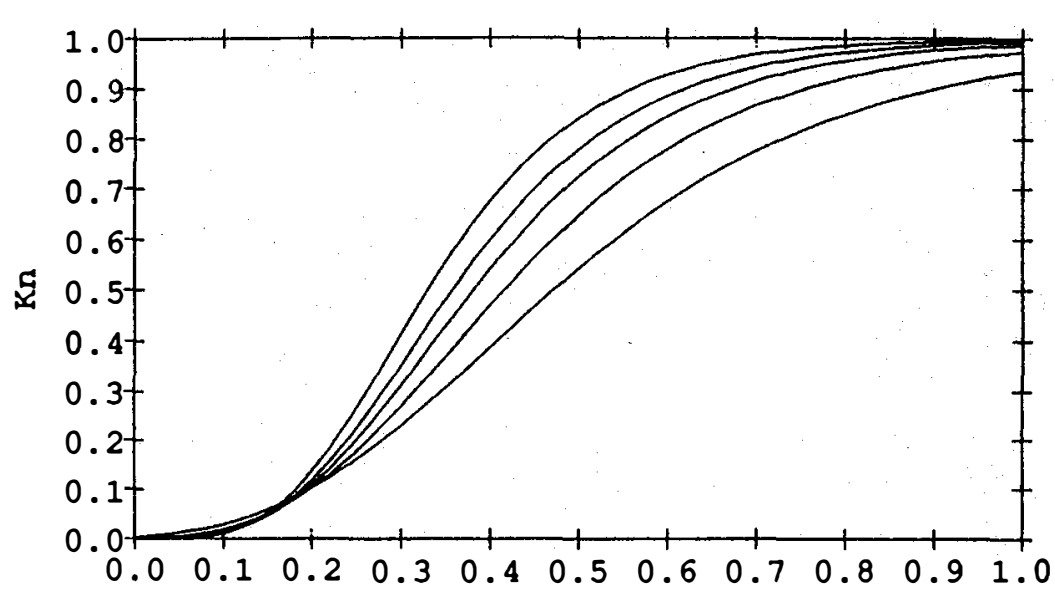

Kt

Vary D

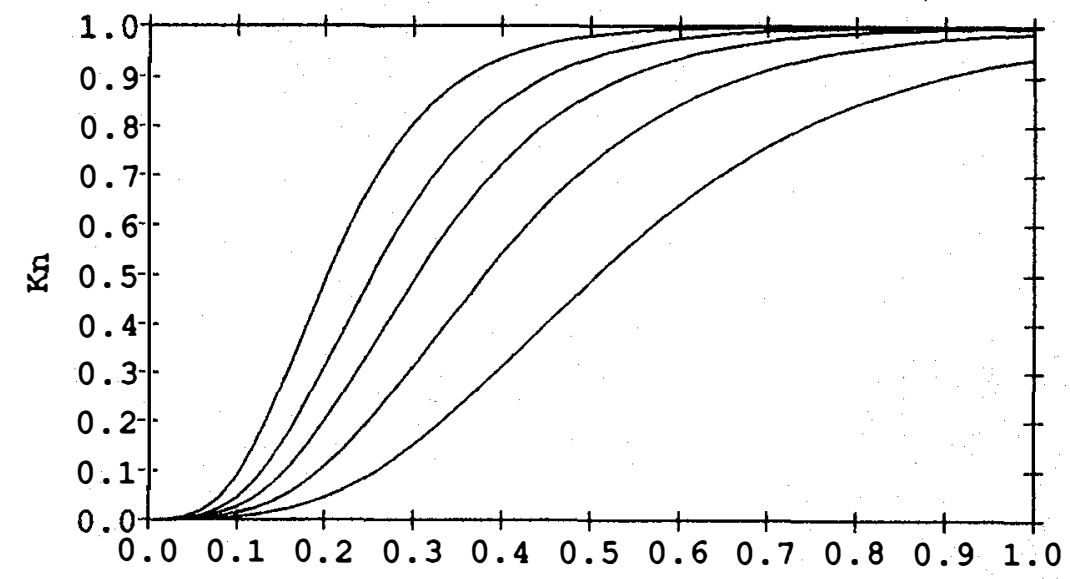

Kt

Figure 5-1. Gompertz curves, illustrating the effects of each of the four coefficients 
Data from a number of locations and months were used to select the boundary shapes that would most accurately represent the effects of the various climate and seasonal conditions found in the United States. The set of six shapes for the left boundary are shown in Figure 5-2. A similar but different set of five shapes developed to fit the right-hand boundary are shown in Figure 5-3. These shapes have proven to be quite adequate for the range of climate and atmospheric conditions found in the United States.

In addition to varying the shapes of the boundaries, it was found that differences in air mass, elevation, and atmospheric variables result in a change in the position of boundaries along the $\mathrm{Kt}$ axis. Therefore, for each of the boundary shapes a family of curves spaced at increments of 0.025 along the Kt axis have boen defined (see Figure 5-4). This provides the capability of varying position along the Kt axis, without changing shape, to achieve the best match with the actual data.

The identification of a boundary requires just three digits. The first digit identifies a shape from 1 through 6 and the next two digits identify the position of that shape from 1 through 20. Thus, the identification of both left and right boundaries requires only six digits. This is discussed further in Chapter 6, Section 6.3 (page 44). These digits are not Gompertz coefficients; they simply identify curve shapes and position.

\subsection{Boundaries for Other Pairs of Components}

All of the plots shown thus far have been for Kt and $\mathrm{Kn}$. Because some measurement stations measure global and diffuse or direct normal and diffuse components, establishing boundaries for these combinations must be considered. However, the boundaries of the scatter plots of solar radiation data in Kt-Kd space or Kd-Kn space cannot be fit by the Gompertz equation. Although a function could undoubtedly have been found to create boundaries for solar radiation data in the $\mathrm{Kt}$-Kd space, there was concern about the added complexity, plus a more serious concern over the consistency of quality control using boundaries in different spaces defined by different functions. In other words, we were concerned that one could not be sure that the same quality control limits were being applied if different boundary functions were used for different radiometric spaces.

Because two components can always be used to produce the third, an investigation was made of the feasibility of using the Kt-Kn space for quality control of any of the three possible pairs of the three components. A representative example of the success of this approach is shown in Figure 5-5. In this instance, all three components were available for Bismarck, ND, for July. The plot of actual global and direct data is matched almost exactly with the plot of global and diffuse data, where the diff use values have been converted to the equivalent direct normal value using the equation, $\mathrm{Kn}=\mathrm{Kt}-\mathrm{Kd}$. A number of station-months were examined for a variety of climates during each of the four seasons, with results similar to those shown in Figure 5-5. Therefore, the same algorithms, boundary functions, and 


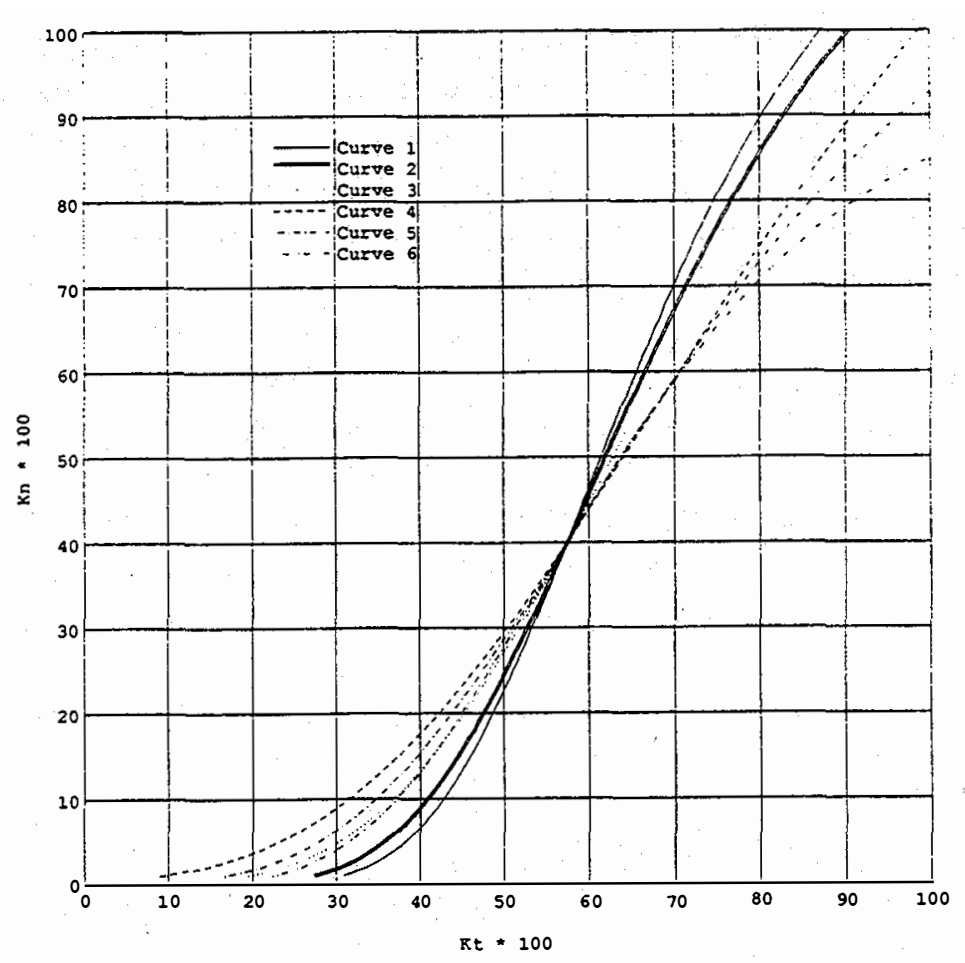

Figure 5-2. Set of six Gompertz curves used to define the shape of the left boundary of K-space plots of solar radiation data

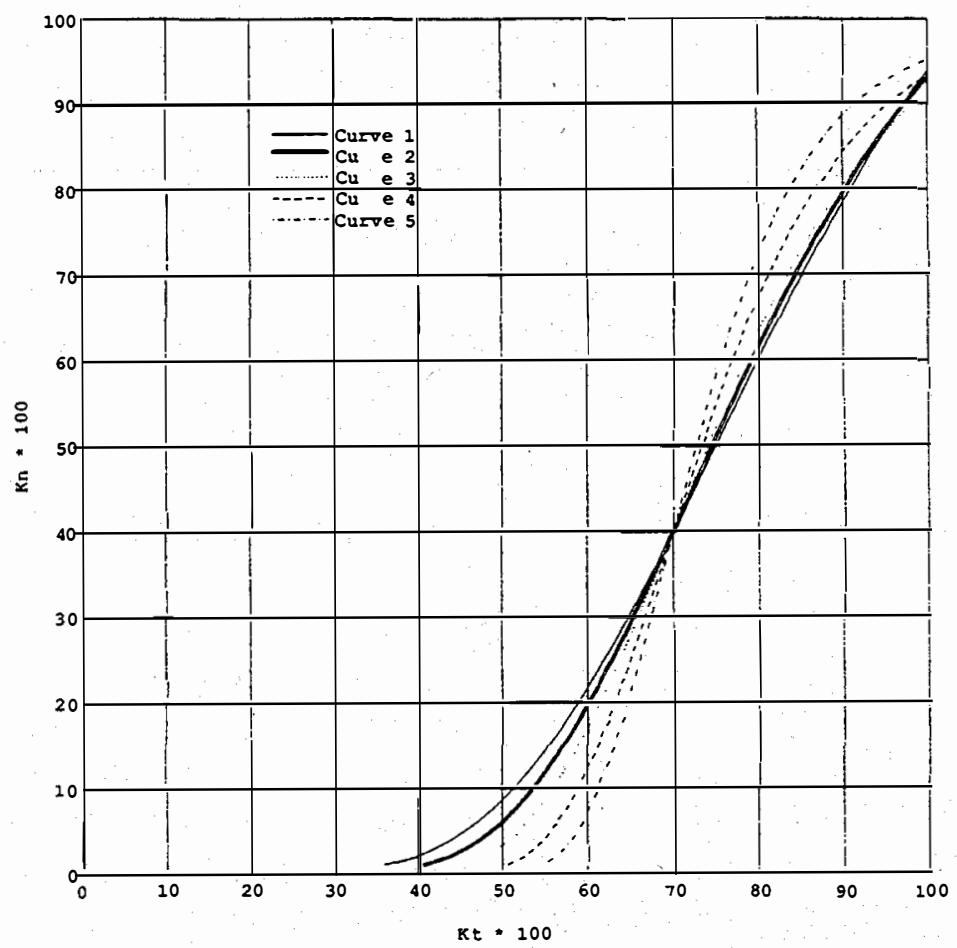

Figure 5-3. Set of five Gompertz curves used to define the shape of the right boundary of K-space plots of solar radiation data

Chapter 5 - 36 


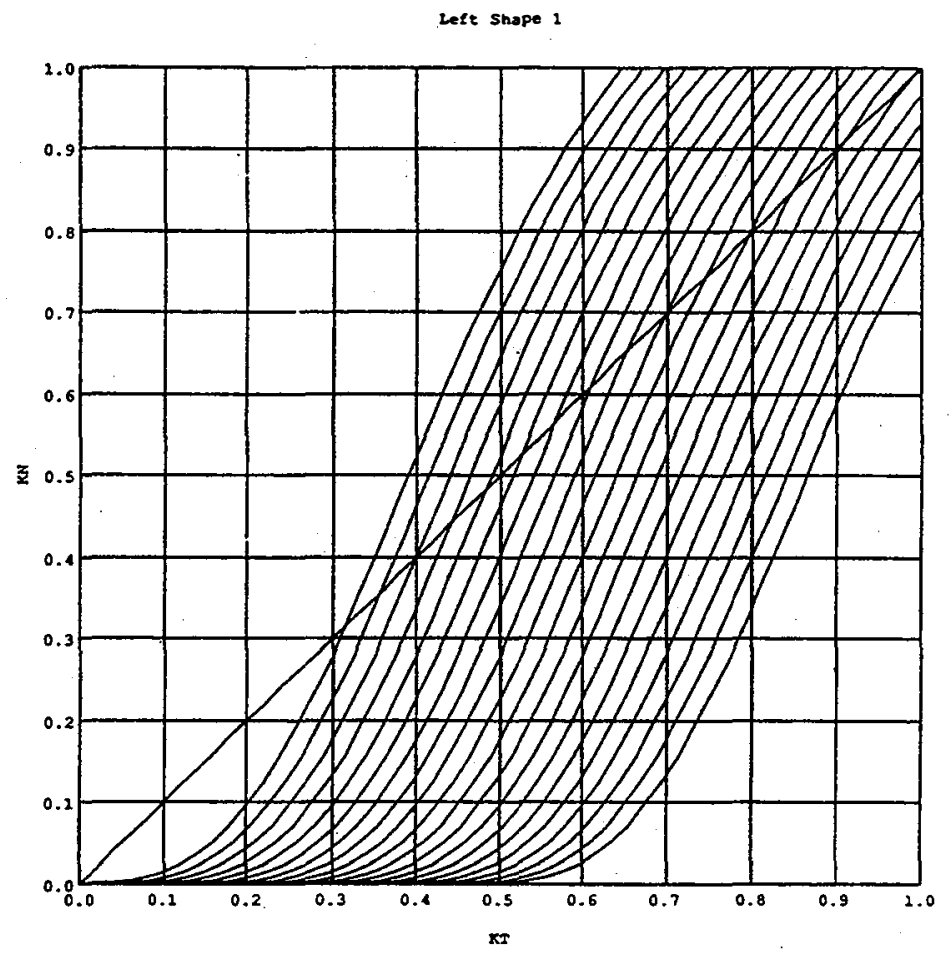

Figure 5-4. Family of Gompertz curves used to define left boundary shape number 1

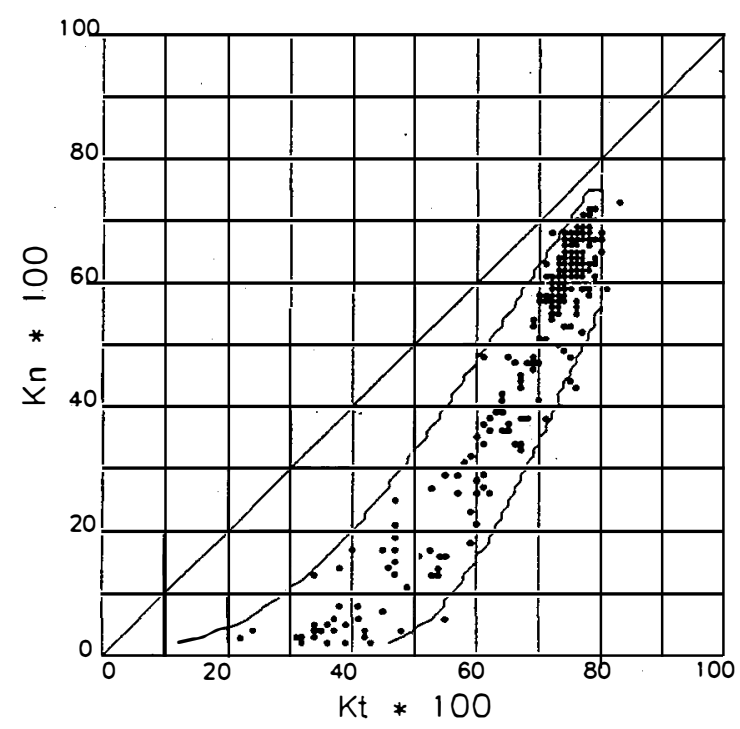

Global-Direct

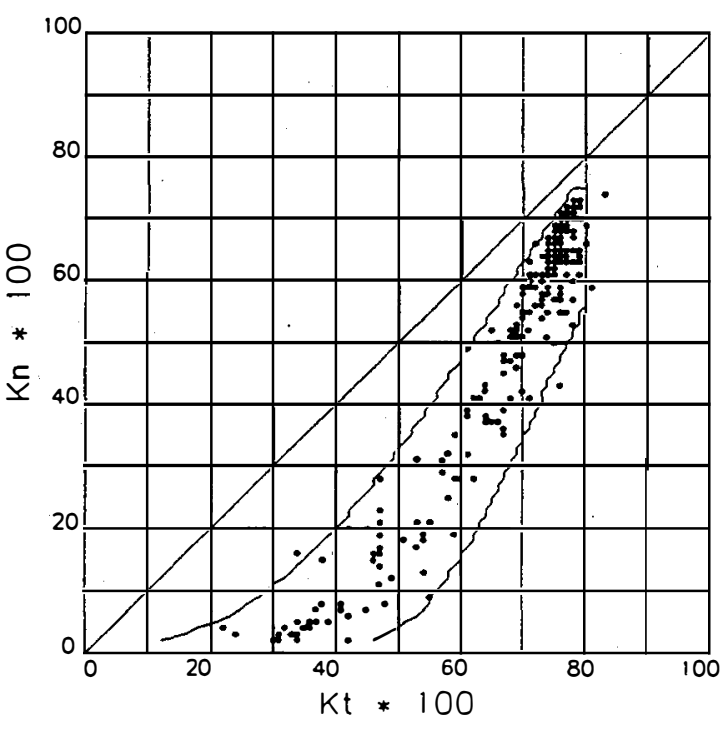

Global-Diffuse

Figure 5-5. Global-direct and global-diffuse scatter plots for Bismarck, ND, for July

Quality Assessment Boundaries - 37 
designation of boundary shapes and positions for a given station-month can be used for any combination of two of the three fundamental components.

\subsection{Boundaries for Other Integration Times}

The ephemeral effects of cloud reflection and cloud focussing were discussed in Chapter 4, Section 4.2.1 (page 24). As a result of these short-lived phenomena, the boundaries defining expected $\mathrm{Kt}$ and $\mathrm{Kn}$ values for a given station-month will change with changes in the integration period of the measurement.

An example of the effect of different integration times is shown in Figure 5-6. The 1-minute data were obtained by Trinity University in San Antonio, TX. They were integrated to form 5-minute, 15-minute, and 1-hour data sets. Therefore, all of the data shown in this figure came from the same 1-minute samples. It should be noted that in San Antonio in July the convective generation of clouds is quite active. From Hahn et al. (1984) we find that the types of clouds are a mixture of cirrus, cumulus, and stratus, in that order relative to frequency of occurrence. Under these cloud conditions, we observe the effects of short-term enhancement of the diffuse component produced by the scattering or focusing of light from nearby clouds. The evidence of this phenomena is greatly reduced with integration times of 15 minutes and has virtually disappeared for the 1-hour data. In other words, the short-term enhancement of the diffuse component is smoothed out by integrating for 1 hour. It is also important to note that a $\mathbf{K}_{\max }$ limit of about 0.9 for the 1-minute data decreases to 0.85 for 5 -minute data, 0.83 for 15 -minute data, and 0.80 for 1 -hour data.

The maximum Kn value and the left boundary for the data are seen to remain fixed for all of these integration times. This indicates that the very clear atmospheric conditions producing high $\mathrm{Kn}$ values are less ephemeral and generally persist for longer than 1 hour.

Under stratus or cirrus cloud cover we see less enhancement of the diffuse and less change in boundary positions for short integration times. Also neither $\mathrm{Kn}_{\max }$ nor $\mathrm{Kt}_{\max }$ undergo significant changes. These results lead us to conclude that changes in boundary positions and $\mathrm{Kt}_{\max }$ with varying integration times will be a function of climate and season. The greater changes, resulting from the enhanced diff use component, will take place during those seasons and at those locations for which cumuloform clouds are frequently observed. It appears that the enhancement of the diffuse component is smaller in magnitude and occurs less frequently with stratus and cirrus cloud forms. 


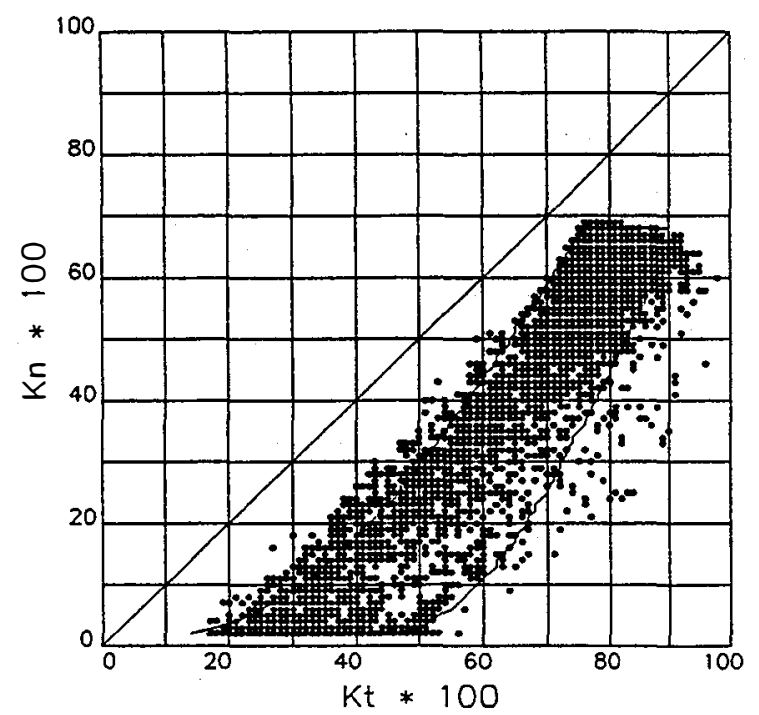

1-minute

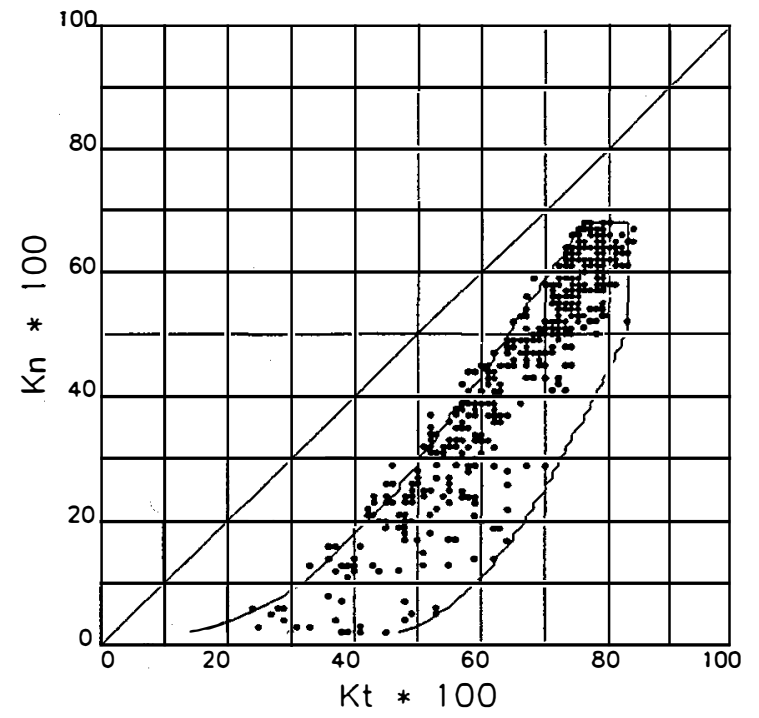

15-minute

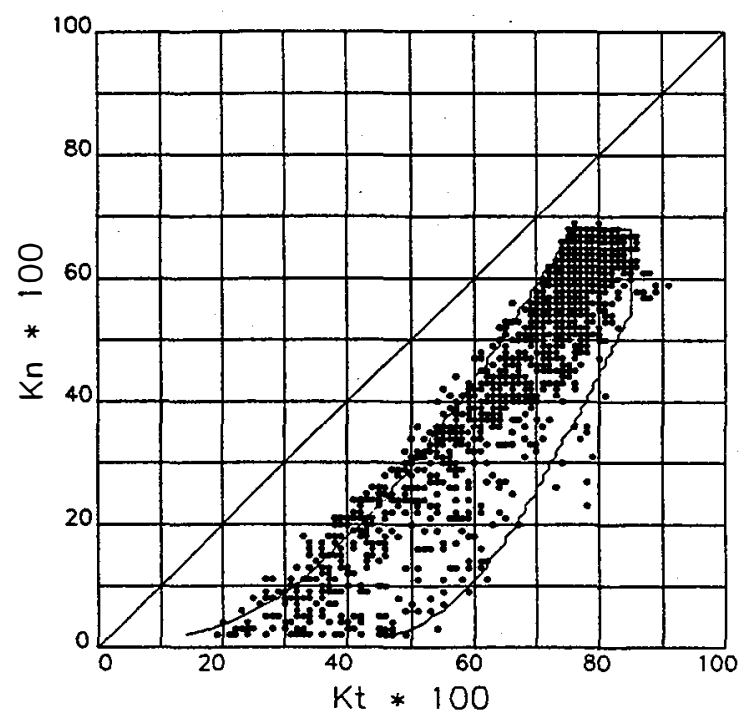

5-minute

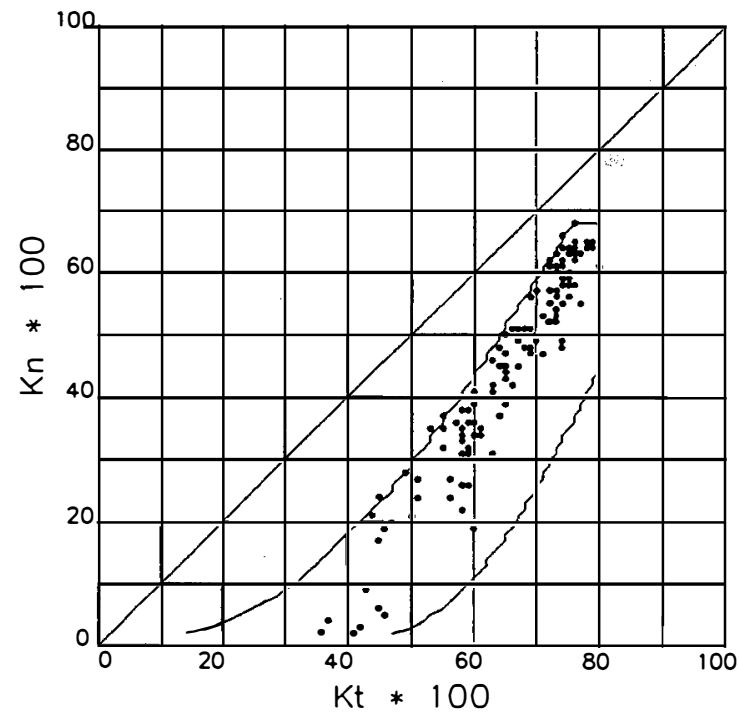

1-hour

Figure 5-6. An illustration of the effects of integration times on K-space scatter plots. Data are from San Antonio, TX, during July. 


\subsection{Summary Comments}

Overall, the effects of different integration times and the effects of different components have had only minor effects on the quality assessment software. The procedure developed appears to be adaptable for almost any combination of components and integration periods. It also appears feasible that global data taken on a tilt could be processed using the same software if the tilt values are converted to global horizontal and direct normal values. Models for making such transformations have been improved in recent years and produce sufficiently accurate results to support this procedure. However, we have not had the opportunity to evaluate this method of quality assessment of tilt data.

\subsection{References}

Hahn, C.J., et. al. (1984). Atlas of Simultaneous Occurrence of Different Cloud Types Over Land. NCAR/TN-241+STR, Boulder, CO: National Center for Atmospheric Research.

Parton, W.J. and Innis, G.S. (1972). Some Graphs and Their Functional Forms, Tech. Report No. 153, Ft. Collins, CO: National Resource Ecology Laboratory, Colorado State University. 


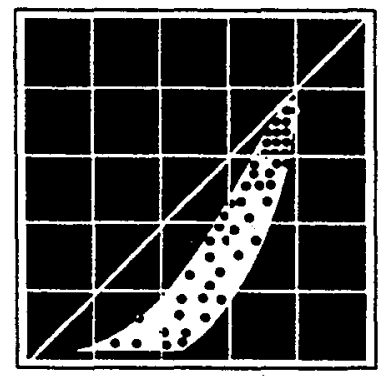

\section{Chapter 6}

\section{A Comprehensive Quality Assessment System}

Before describing the quality control procedures and software, it should be noted that the departures of measured data from expected limits and boundaries are calculated relative to a $\mathrm{K}$-value of 1.0. In other words, if a data point fails to meet a quality control criteria in the $\mathrm{Kt}-\mathrm{Kn}$ space by 0.03 , this is designated as a $3 \%$ error in $\mathrm{K}$-space. This error is assigned regardless of whether the expected value of $\mathrm{K}$ is 0.9 or 0.09 . Given that the solar constant equals $1367 \mathrm{~W} / \mathrm{m}^{2}$, a $1 \%$ error in $\mathrm{Kn}$ will equal $13.67 \mathrm{~W} / \mathrm{m}^{2}$. The absolute values of the errors in $\mathrm{Kt}$ and $\mathrm{Kd}$ will be a function of zenith angle, because these $\mathrm{K}$-values are computed relative to solar irradiance on a horizontal surface outside the atmosphere.

\section{Establishing Limits and Boundaries}

The one-component max-min limits and the two-component Gompertz boundaries must be established on the basis of existing good quality data. A computer program (QCFIT) was written to help determine the limits and boundaries. QCFIT, described in Chapter 8 (page 97), QCFIT Software Users Guide, automatically selects the Gompertz boundaries using a criterion whereby the selection of tighter fitting boundaries results in a greater percentage increase in errors than the percentage decrease in the acceptable area between the boundaries. When a large number of data points is available (greater than 300) QCFIT usually does a good job of selecting the boundaries. This is particularly true when there are no anomalous data outside the right-hand boundary and when the data uniformly populate the area between the two boundaries. Under other circumstances, QCFIT provides for operator editing so that the human eye and brain can select boundaries that are most reasonable for the climate and season under consideration.

It should be noted that in general, anomalous data resulting from cloud scattering and focusing are left outside the right-hand boundary and will be flagged accordingly. This results from the error versus area comparison and the criterion that boundaries will be set to produce up to $5 \%$ of Type 1 errors. Anomalous data rarely exceed this percentage, except under high surface albedo conditions (snow cover). 
$\mathrm{Kt}_{\max }$ and $\mathrm{Kn}_{\max }$ are initially set by QCFIT to values of 0.93 and 0.85 , respectively. These values are likely too high for all but 1- to 5-minute data under certain climate and seasonal conditions. The user must then use the QCFIT edit mode to adjust these values to fit the data. This manual operation is necessary because we want $\mathrm{Kt}_{\max }$ and $\mathrm{Kn}_{\max }$ to encompass the bulk of valid data but not anomalous values. Hence, an automatic search for maximum $\mathrm{Kt}$ and $\mathrm{Kn}$ values cannot be used.

This forces the user of QCFIT to visually examine the data and boundary fits at least once, which is highly desirable.

Setting a value for $\mathrm{Kd}_{\max }$ presented an interesting problem. $\mathrm{Kt}_{\max }-\mathrm{Kd}_{\max } \neq$ $\mathrm{Kn}_{\max }$ because $\mathrm{Kt}_{\max }$ and $\mathrm{Kn}_{\max }$ occur under cloudless skies with low turbidity and water vapor. $\mathrm{Kd}_{\max }$, however, is most likely to occur when turbidity and water vapor are high, with or without some cloud cover.

Using a visual examination of a scatter plot of data to set $\mathrm{Kd}_{\max }$ would require a scatter plot of $\mathrm{Kd}$ versus $\mathrm{Kt}$ or $\mathrm{Kn}$. This would unnecessarily complicate QCFIT and presents the possibility of selecting inconsistent limits and boundaries for the three components.

From an examination of scatter plots and Gompertz boundaries in Kt-Kn space, it was noted that the right-hand boundary can be used to set the maximum Kd value. For any point on the boundary, the equation $\mathrm{Kd}=\mathrm{Kt}-\mathrm{Kn}$ can be used to determine the highest $\mathrm{Kd}$ value likely for the associated $\mathrm{Kt}$ and $\mathrm{Kn}$ values. It was subsequently determined that $\mathrm{Kd}_{\max }$ occurs at that point where the slope of the right-hand boundary equals 1.0. If the slope of the boundary is always greater than $1.0, \mathrm{Kd}_{\max }$ is defined by the intercept of the right-hand boundary with the Kt axis.

\subsection{Designating Limits and Boundaries}

The inherent simplicity and efficiency of SERI_QC1 is indicated by Table 6-1, the QC-zero file for Albuquerque, NM. This table contains all of the information needed to perform quality assessments for solar radiation data from Albuquerque. All limits and boundaries are designated for each month and for each of the three air mass ranges defined in Table 4-1 (page 25). QC-zero files are created by. QCFIT and used by SERI_QC1 (see Chapters 7, 8, and 9 for details).

The group of five two-digit numbers following the month name defines $\mathrm{Kn}_{\max }$ and $\mathrm{Kt}_{\max }$ for low air mass. (As noted in Section 4.2.2, page 25, these $\mathrm{K}_{\max }$ limits decrease in fixed steps for the medium and high air mass ranges.) Division of these numbers by 100 converts them to $K_{\max }$ values. The first number defines $\mathrm{Kn}_{\max }$ for all integration times. The next four numbers set $\mathrm{Kt}_{\max }$ limits for 1-minute, 5-minute, 15-minute and 1-hour data, in that order. In this example, only 1-hour data were available and the limit for shorter integration times is set to 00 , indicating they are undefined. 
Table 6-1. QC-zero File for Albuquerque, NM

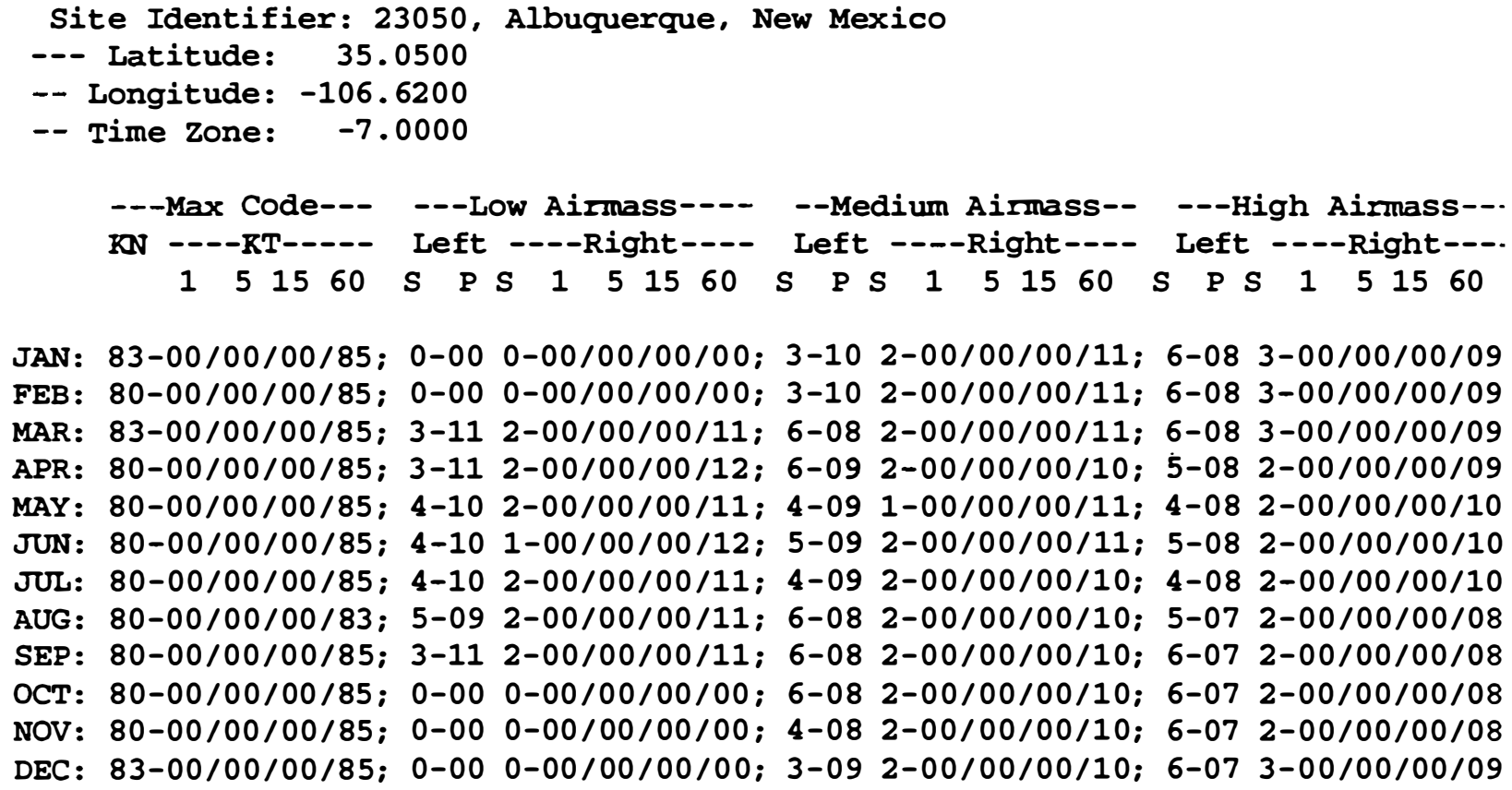

The next three groups of seven numbers designate boundary shapes and positions for low, medium, and high air mass ranges, in that order. The first number (singledigit) in each group of seven identifies the shape of the left boundary, according to the curve numbers in Chapter 5, Figure 5-2 (page 36). The second number (twodigit) identifies the position of the curve along the Kt axis. The shape and position of the left-hand boundary do not appear to change with integration time. Hence, the first two numbers designate the left boundary for all data, regardless of integration time.

The center or position 10 for each of the left boundary shapes is positioned so that it goes through the point $\mathrm{Kt}=0.575, \mathrm{Kn}=0.4$. The other curves in the family move along the Kt axis in increments of 0.025 .

The third number (single-digit) in each group of seven designates the shape of the right boundary (see Figure 5-3, page 36). The fourth number (two-digit) designates the position along the $\mathrm{Kt}$ axis for 1 -minute data. Position 10 indicates a curve that passes through the point $\mathrm{Kt}=0.7, \mathrm{Kn}=0.4$.

Our observations indicate that the right boundary shapes do not change with integration time but positions do change. Hence, the last three two-digit numbers designate curve positions for the right-hand boundary for 5-minute, 15-minute, and 1-hour data, respectively. This sequence is repeated for medium and high air mass ranges. 


\subsection{SERI_QC1 Flow Diagram}

Once QC-zero files have been established for each station, data can be routinely processed. Quality assessment is usually performed in conjunction with other processing required to establish data archives. Therefore, a subroutine, SERI_QC1, will be called by a general processing program to invoke the quality assessment algorithms. A chart of the flow of data through SERI_QCl is shown in Figure 6-1.

During each data test, two-digit flags are set for each of the three solar radiation components to indicate the result of the test. These flags are returned by the SERI_QC1 subroutine to the calling program, which will use them according to the purpose of the program. For example, the three-component input data for SERI_QC1 might appear as

$$
653.89896 .1166 .94
$$

where the components are global horizontal, direct normal, and diffuse horizontal, and the units are W/m $/ \mathrm{m}^{2}$. SERI_QCl returns three assessment flags, each associated with the corresponding input data. For example:

$$
030303
$$

The flag of 03 indicates the data passed the three-component test given by equation 4-4 $(\mathrm{Kt}=\mathrm{Kn}+\mathrm{Kd})$. The method of passing data to and receiving flags from the SERI_QC1 subroutine is detailed in Chapter 9.

Station identification and date and time checks are made first, because all computations and comparisons are specific to a given location, day, and hour. This check is followed by missing data tests that determine which of the solar radiation components are present. Missing components are assigned a flag 99. Next, the nighttime hours are identified and special nighttime tests are performed (values should be close to zero).

For solar zenith angles greater than $80^{\circ}$ (at the middle of the measurement period) special "twilight" one-component max-min empirical limit tests are made. All $\mathrm{K}$-minimum limits are set to zero. At zenith angles greater than $80^{\circ}$, the high rate of change of solar radiation under clear sky conditions increases the uncertainty of hourly averages. Other factors affecting the uncertainty of measurements at large zenith angles include the leveling of the pyranometers, tracking errors due to refraction in the earth's atmosphere, objects on the horizon occluding the direct beam, and irregularities in the zenith angle response of the pyranometers. Furthermore, for zenith angles greater than $82.5^{\circ}$, hourly averages may actually be for periods less than 60 minutes. 


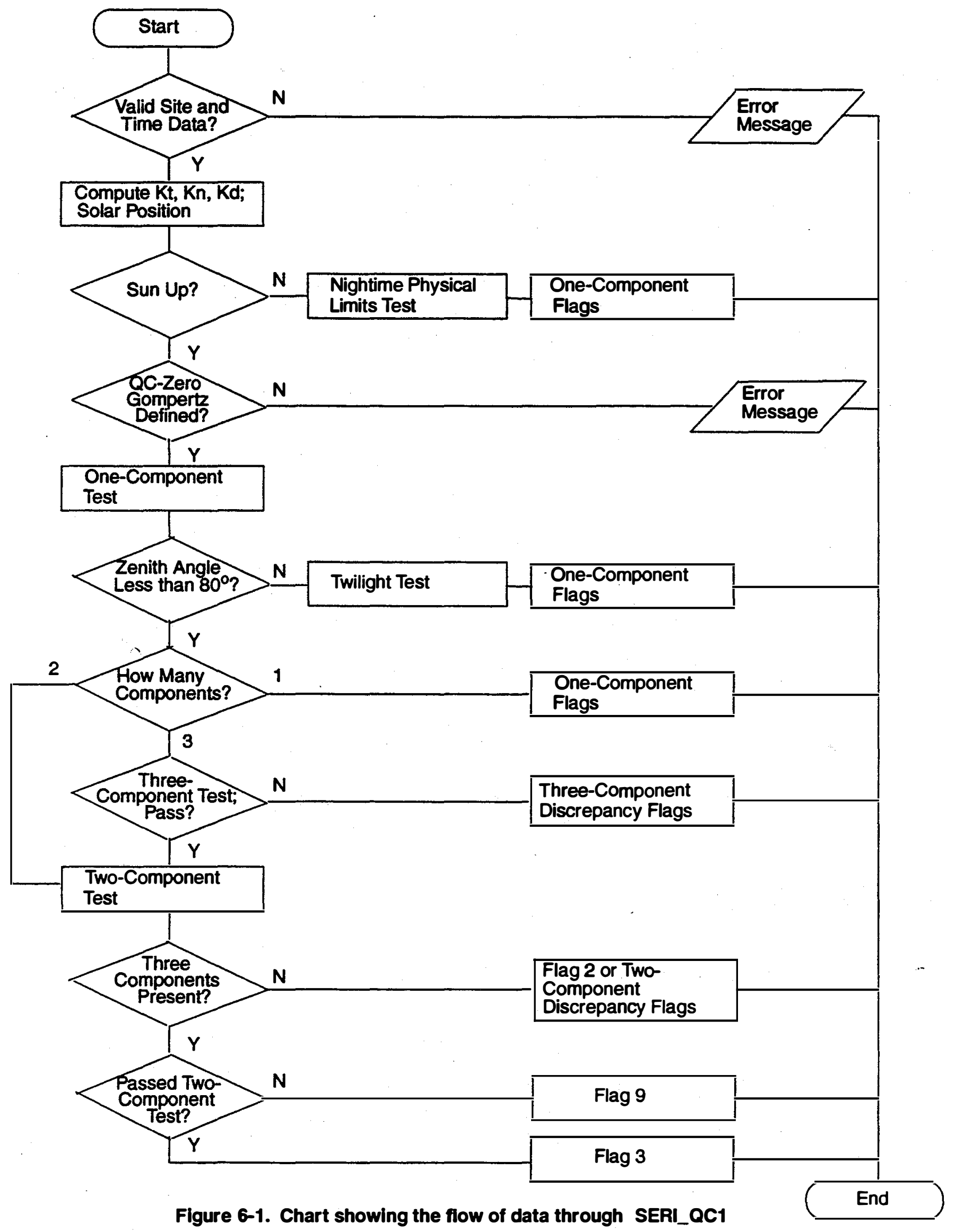

Quality Assessment System - 45 
For solar zenith angles less than or equal to $80^{\circ}$, one-component empirical limit tests are performed first. These are tests against minimum and maximum $\mathrm{Kt}, \mathrm{Kn}$, and Kd values based upon previous measurements (see Section 9.3.3). The minimum limit for the direct normal component is $-10 \mathrm{~W} / \mathrm{m}^{2}$, which allows for small negative values that can occur when a bright sun is suddenly obscured by clouds. If only one component is present, the tests are terminated at this point. If two components are present, but one of them fails the one-component test, they both will be tested against the one-component limits and the tests will be terminated.

If both components pass the one-component tests, they are then compared to determine if $\mathrm{Kn}>\mathrm{Kt}$ (a physical impossibility). If $\mathrm{Kn}>\mathrm{Kt}$, the tests are terminated with flags that indicate the error; if not, two-component empirical tests are performed. New flags are set to indicate the results of the two-component tests. If three components are present, a redundancy test is made. The redundancy test consists of the comparison of the measured $\mathrm{Kt}$ value with a $\mathrm{Kt}$ value estimated from the equation

$$
K t=K n+K d
$$

Because the three-component redundancy test is considered to be more accurate and reliable than the one- and two-component tests, these flags will take precedence over previously set flags, unless the combination of two-component and three-component tests indicate the presence of anomalous conditions.

Anomalous conditions are defined as those situations where the three-component redundancy test is passed, whereas the two-component empirical limit test is failed. This most often occurs when the data points fall outside the right-hand Gompertz boundary. As noted in Section 4.2.1 (page 24), these data appear to be the result of reflection or focusing from nearby clouds and/or a high surface albedo. If the combined two-component and three-component tests indicate anomalous data, a special flag 09 (see Section 6.4) is set.

\subsection{Tests and Flags}

\subsubsection{One-Component Tests}

Table 6-2 describes the use and meaning of the two-digit flags that are set by SERI_QC1. A flag of 00 indicates the data have not undergone quality assessment. A flag of 01 indicates that the data passed a one-component test. Most often this means there was only one component present, but there are exceptions to that. Notably, if the solar zenith angle is greater than $80^{\circ}$, only one-component tests are performed. 
Table 6-2. Flagging Convention for SERI_QC1

\begin{tabular}{|c|c|c|}
\hline Flag & & Description \\
\hline $\mathbf{0 0}$ & \multicolumn{2}{|c|}{ Untested (raw data) } \\
\hline 01 & \multicolumn{2}{|c|}{ Passed one-component test; data fall within max-min limits of $\mathrm{Kt}, \mathrm{Kn}$, or $\mathrm{Kd}$} \\
\hline 02 & \multicolumn{2}{|c|}{ Passed two-component test; data fall within 0.03 of the Gompertz boundaries } \\
\hline 03 & \multicolumn{2}{|c|}{$\begin{array}{l}\text { Passed three-component test; data come within } \pm 0.03 \text { of satisf ying } \\
\mathrm{Kt}=\mathrm{Kn}+\mathrm{Kd}\end{array}$} \\
\hline 04 & \multicolumn{2}{|c|}{ Passed visual inspection; not used by SERI_QCl } \\
\hline 05 & \multicolumn{2}{|c|}{ Failed visual inspection; not used by SERI_QCl } \\
\hline 06 & \multicolumn{2}{|c|}{ Value estimated; passes all pertinent SERI_QCl tests } \\
\hline 07 & \multicolumn{2}{|c|}{ Failed one-component test; lower than allowed minimum } \\
\hline 08 & \multicolumn{2}{|c|}{ Failed one-component test; higher than allowed maximum } \\
\hline 09 & \multicolumn{2}{|c|}{ Passed three-component test but failed two-component test by $>0.05$} \\
\hline \multirow[t]{3}{*}{$10-93$} & \multicolumn{2}{|c|}{$\begin{array}{l}\text { Failed two- or three-component tests in one of four ways. } \\
\text { To determine the test failed and the manner of failure (high or low), examine } \\
\text { the remainder of the calculation (flag }+2) / 4 \text {. }\end{array}$} \\
\hline & $\begin{array}{l}0 \\
1 \\
2 \\
3\end{array}$ & $\begin{array}{l}\text { Parameter too low by three-component test }(\mathrm{Kt}=\mathrm{Kn}+\mathrm{Kd}) \\
\text { Parameter too high by three-component test }(\mathrm{Kt}=\mathrm{Kn}+\mathrm{Kd}) \\
\text { Parameter too low by two-component test (Gompertz boundary) } \\
\text { Parameter too high by two-component test (Gompertz boundary) }\end{array}$ \\
\hline & \multicolumn{2}{|c|}{$\begin{array}{l}\text { The magnitude of the test failure (distance in K-units) is determined from: } \\
d=(\text { INT }(\text { flag }+2) / 4) / 100 \text {. } \\
\text { Examples and further discussion of the meaning of flags } 10-93 \text { are given in the text in } \\
\text { this chapter and in Section } 9.3 \text {, page } 153 \text {. }\end{array}$} \\
\hline $94-97$ & \multicolumn{2}{|c|}{$\begin{array}{l}\text { Data fall into a physically impossible region where } \mathrm{Kn}>\mathrm{Kt} \text { by } \mathrm{K} \text {-space distances of } \\
0.05 \text { to } 0.10(94), 0.10 \text { to } 0.15(95), 0.15 \text { to } 0.20(96) \text {, and } \geq 0.20(97) \text {. }\end{array}$} \\
\hline 98 & \multicolumn{2}{|l|}{ Not used } \\
\hline 99 & \multicolumn{2}{|l|}{ Missing data } \\
\hline
\end{tabular}


The maximum limits determined by QCFIT for $\mathrm{Kt}$ and $\mathrm{Kd}\left(\mathrm{Kt}_{\max }\right.$ and $\left.\mathrm{Kd}_{\max }\right)$ have been increased by 0.1 for routine data processing. This was done to accommodate the effects of cloud reflections, focusing, and high surface albedos. Essentially, these increases in one-component limits account for most of the data falling in the A, B, D, and E regions shown on Figure 4-6 (page 27). The maximum limit for $\mathrm{Kn}$ is not increased over $\mathrm{Kn}_{\max }$, because there is no physical basis for such an increase. Experience confirms this decision.

These increases in maximum limits for $\mathrm{Kt}$ and $\mathrm{Kd}$ will likely increase Type 2 errors but they should decrease Type 1 errors associated with one-component tests. This was judged to be the best compromise and better than the physical limits of zero and ETR. If the one-component tests fail, flags of 07 (too low) and 08 (too high) are set.

\subsubsection{Two-Component Tests}

A flag of 02 indicates the data passed a two-component Gompertz boundary test. Actually, a distance of 0.03 (tolerance zone) is allowed outside the boundaries set by QCFIT before any "test failed" flags are set. This tolerance zone recognizes the general uncertainties associated with solar radiometry and the calibration of solar radiometers (Myers 1989).

When the distance normal to the boundary exceeds 0.03 , flags from [12-92, 4] or [13-93, 4] (incrementing by 4 over the range indicated) will be set. As indicated in Table 6-2, these flags indicate the test that was performed, the manner of the failure (high or low), and the distance from the boundary to the data point (magnitude of the failure).

For example, flags $\mathbf{4 4}$ or $\mathbf{4 5}$ should be interpreted in the following manner.

1. The "remainder" of the division $(44+2) / 4$ is 2 . From Table $6-2$ we note that this means the parameter is judged to be too low by a two-component test. Similarly, a flag of 45 yields a remainder of 3 , indicating the value is too high.

2. From the calculations $d=(\operatorname{INT}(44+2) / 4) / 100=0.11$ and $d=(\operatorname{INT}(45+$ 2)/4) $100=0.11$ we know that the data point lies outside one of the Gompertz boundaries by a distance of 0.11 (K-units).

3. If the flag of $\mathbf{4 4}$ has been assigned to a global horizontal component, this means the data point lies to the left of the left-hand boundary. If this were a global-direct data point, the direct normal component would be assigned a flag of 45 , indicating it is likely too high. If it were a global-diffuse data point, the diffuse horizontal component would be assigned a flag of 44 , indicating it is probably too low. 
4. If the flag of $\mathbf{4 4}$ has been assigned to a direct normal component, this means the data point lies to the right of the right-hand boundary. If this were a global-direct data point, the global horizontal component would be assigned a flag of 45 , indicating it is likely too high. If it were a directdiffuse data point, the diffuse horizontal component would be assigned a flag of 45 , indicating it is likely too high.

Note that in each instance, the assignment of flags to individual components is based on the assumption that the other component has been accurately measured. Any errors resulting from this assumption are mitigated in part by calculating the normal distance to the boundary rather than the horizontal and vertical distances, which are always larger. Because we are never privileged to know the "truth" this method of calculating distances and assigning flags seems to provide the best compromise. Flags of 92 or 93 are assigned when the distance from the boundary is $\geq 0.23$.

\subsubsection{Three-Component Tests}

A flag of 03 indicates that $\mathrm{Kt}$ lies within the range $\mathrm{Kt}^{*} \pm 0.03$, where $\mathrm{Kt}$ is a measured value and $\mathrm{Kt}^{*}$ has been estimated from the equation $\mathrm{Kt}^{*}=\mathrm{Kn}+\mathrm{Kd}$. A flag of 03 will be assigned to any data point that satisfies the above criteria and that also passed the two-component test. If these criteria are satisfied but the data point failed a previous two-component test by more than 0.05 , a flag of 09 is assigned to all three components of the data point. The only flag 09 data that we have observed lie to the right of the right-hand boundaries and are presumed to be the result of cloud reflections, focusing, and/or high surface albedo. Hence, flag 09 has been called "strange, but probably true." It is possible, but unlikely, that flag 09 data beyond the $\mathrm{Kt}_{\max }+0.1$ limit will be observed.

Flags of [10-90,4] and [11, 91, 4] will be found almost anywhere to the right of the diagonal $(0,0-1,1)$ that divides $\mathrm{Kt}-\mathrm{Kn}$ space into physically possible and impossible regions. Because three component tests are more reliable, threecomponent flags are retained regardless of previous one- or two-component results.

As noted for two-component tests, the even or odd flag (for flags $\geq 10$ ) again determines whether the component is presumed to be low or high, respectively. Furthermore, the K-distance by which a component may be in error is calculated using the equation

$$
\mathrm{d}=[\mathrm{INT}(\mathrm{flag}+2) / 4] / 100
$$

As for two-component tests, flags are assigned based on the assumption that the other two components have been accurately measured. 
For example, if $\mathrm{Kt}=\mathrm{Kt}^{*}-0.11$, the global horizontal component will be assigned a flag of 42 and the direct nornal and diffuse horizontal components will be assigned flags of 43 . These flag assignments will be reversed if $K t=K t^{*}+0.11$.

This completes the description of the four flags $(42,43,44,45)$ that may be assigned when two- or three-component data exceed maximum or minimum limits by a distance of 0.11 in $\mathrm{K}$-units.

\subsection{References}

Myers, D.R. (1989). “Application of a Standard Method of Uncertainty Analysis to Solar Radiometer Calibrations," Proc. 1989 Annual ASES Conference, Denver, CO. 


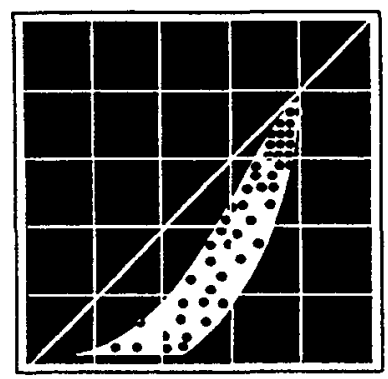

Part 2

Users Guide

\section{Contents}

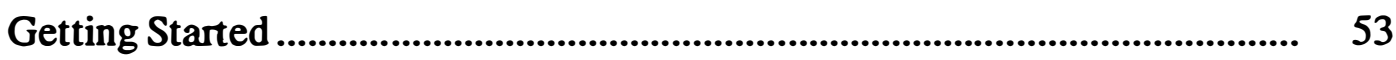

Chapter 7 - The SERI QC Software and Hardware Environment ..................... 83

Chapter 8 - QCFIT Software Users Guide........................................................ 97

Chapter 9 -SERI_QC1 Software Users Guide ............................................... 145

\section{Overview}

Part 2 of this manual gives you information about the SERI QC software so you can develop procedures for solar radiation data processing and quality assessment. The following summarizes the contents of Part 2:

- The getting started tutorial explains software installation and provides a hands-on introduction to the concepts of boundary selection with QCFIT and the use of the SERI_QC1 subroutine.

- Chapter 7 outlines hardware requirements and explains the fundamental data structures of the software.

- Chapter 8 contains detailed instructions for the use of the QCFIT boundary fitting software.

- Chapter 9 explains the SERI_QC1 assessment algorithms and how to call the subroutine from a FORTRAN program. 



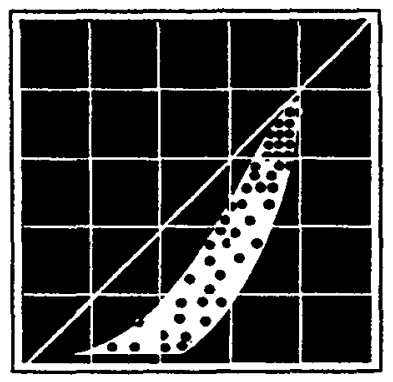

\section{Getting Started}

\section{Overview}

SERI QC is a computer software package for assessing the quality of solar radiation data. This Getting Started section tells you how to install the software and guides you through a sample session using the QCFIT software. In addition, you will compile and link a sample program that calls the SERI_QC1 subroutine. Chapters 7, 8 , and 9 go into greater detail about each of the steps specified here. This gettingacquainted section is not a substitute for other information in this manual. 


\section{What You Need}

To run this software, you need:

- A PC with a math coprocessor and a VGA color monitor

- The SERI QC distribution disk

- About 1 megabyte of free disk space

- A FORTRAN-77 compiler that supports the following language extensions:

- Variable names may be longer than 6 characters

- Variable names may include the underscore character ("_")

- Variables may be initialized with slash characters (“ $\Gamma$ ”) when declared

- Integer*2 data type.

Note: The compiler is required to compile the SERI_QC1 source code and link it with your application software.

The distribution disk contains the following files:

Root Directory

QCFIT.EXE

QCFIT.FOR

SERI_QC1.FOR

SERI_QC1.OBJ

HPLJ.BIN

EPS-P.BIN

ABQ06A.DAT

ABQ06B.DAT

ABQ06C.DAT

QA_ABQ.FOR
QCFIT executable

QCFIT source

SERI_QC1 subroutine (source)

SERI_QC1 subroutine (object)

Printer driver for Hewlett-Packard LaserJet III or 4 printer

Printer driver for PostScript printer

Sample input data (Albuquerque: June 78-80)

Sample input data (Albuquerque: June 81-85)

Sample input data (Albuquerque: June 88-90)

Sample FORTRAN program

IFILES.OC0 directory

S_?????.QC0 QC-zero files for several NOAA stations 


\section{How to Start}

\section{Step 1. Create a SERI QC directory}

Use the MD (Make Directory) command to create a destination directory on your hard drive. For example:

\section{ISERIQC}

You can create this directory anywhere in your directory tree and name it as you wish.

Log to this new directory with the CD command. For example:

\section{ISERIQC}

\section{Step 2. Copy the filles}

Insert the distribution disk into a floppy disk drive and use the DOS XCOPY command to copy the files and subdirectories from the floppy drive (drive $A$ in this example) to the subdirectory just created on your hard drive:

\section{XCOPY A:*.*/S}

If your floppy drive is other than drive $A$, issue the command with the correct drive designator substituted for $A$. If you are using a version of DOS older than 3.x, the XCOPY command is not supported, and you will need to copy the root directory and FILES.QC0 subdirectory with two copy commands.

The software is now installed and ready for use as detailed in this manual. If you wish, try the sample session in the following section to become familiar with the software operation. 


\section{Sample Session}

The goal of this session is to use the QCFIT program to determine expected values for the National Oceanic and Atmospheric Administration's (NOAA) solar monitoring site at Albuquerque, $\mathbf{N M}$, by examining sample historical solar radiation data in the ABQ06A.QCF file included on the distribution disk. Once th. expected values have been determined, program $Q A \_A B Q$.FOR will be used to assess the quality of data taken in other years.

The sample data file, ABQ06A.QCF, contains solar radiation data from the Albuquerque site for the month of June from 1978, 1979, and 1980. Each record in this file has date/time fields, and hourly global horizontal, direct normal, and diffuse horizontal irradiance data in watts per square meter. The first few lines of the file are shown in Figure GS-1.

The format of this file is explained in Chapter 8. Your data format need not be the same, but if it is not, you must specify the format according to the instructions in Section 8.2.2 (page 102), "Modifying the QCFIT Defaults."

$\begin{array}{rrrrrrrr}78 & 6 & 1 & 1 & 0 & 0.00 & 0.00 & 0.00 \\ 78 & 6 & 1 & 2 & 0 & 0.00 & 0.00 & 0.00 \\ 78 & 6 & 1 & 3 & 0 & 0.00 & 0.00 & 0.00 \\ 78 & 6 & 1 & 4 & 0 & 0.00 & 0.00 & 0.00 \\ 78 & 6 & 1 & 5 & 0 & 1.11 & 1.11 & 9900.00 \\ 78 & 6 & 1 & 6 & 0 & 65.00 & 223.06 & 9900.00 \\ 78 & 6 & 1 & 7 & 0 & 251.12 & 603.91 & 9900.00 \\ 78 & 6 & 1 & 8 & 0 & 468.91 & 758.09 & 9900.00 \\ 78 & 6 & 1 & 9 & 0 & 671.97 & 821.98 & 9900.00 \\ 78 & 6 & 1 & 10 & 0 & 843.92 & 863.09 & 138.06 \\ 78 & 6 & 1 & 11 & 0 & 966.15 & 888.09 & 126.12 \\ 78 & 6 & 1 & 12 & 0 & 1033.10 & 913.09 & 121.12 \\ 78 & 6 & 1 & 13 & 0 & 1026.99 & 891.98 & 126.95 \\ 78 & 6 & 1 & 14 & 0 & 948.09 & 821.14 & 158.90 \\ 78 & 6 & 1 & 15 & 0 & 833.92 & 746.97 & 186.12 \\ 78 & 6 & 1 & 16 & 0 & 345.01 & 183.90 & 191.95 \\ 78 & 6 & 1 & 17 & 0 & 498.08 & 555.02 & 191.95 \\ 78 & 6 & 1 & 18 & 0 & 233.90 & 415.02 & 91.11 \\ 78 & 6 & 1 & 19 & 0 & 88.89 & 233.06 & 51.95 \\ 78 & 6 & 1 & 20 & 0 & 6.11 & 8.06 & 9900.00 \\ 78 & 6 & 1 & 21 & 0 & 0.00 & 0.00 & 0.00 \\ 78 & 6 & 1 & 22 & 0 & 0.00 & 0.00 & 0.00 \\ 78 & 6 & 1 & 23 & 0 & 0.00 & 0.00 & 0.00 \\ 78 & 6 & 1 & 24 & 0 & 0.00 & 0.00 & 0.00\end{array}$

Figure GS-1. Sample data file 


\section{Starting the Program}

Log to the directory in which you installed the software, then run QCFIT by typing

QCFIT $<$ CR $>$ (Note that $<\mathrm{CR}>$ indicates a Carriage Retum or Enter)

The QCFIT opening screen and component selection prompt appears:

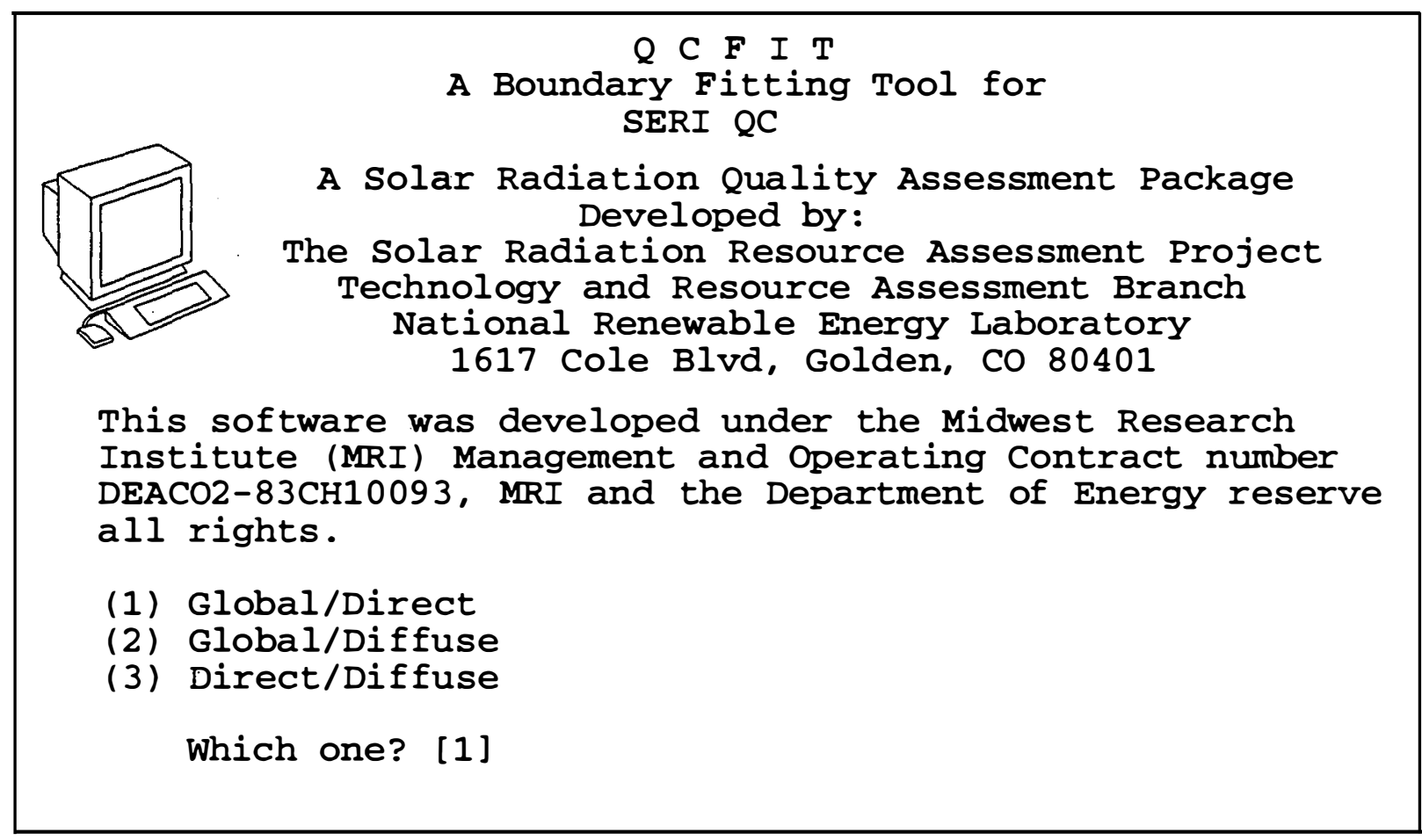

\section{Choosing Solar Components}

To examine the global and direct data in the file (although later you may want to examine the same data file using the other combinations of components), enter:

$1<\mathrm{CR}>$

Note that default responses appear in brackets (in this case [1]), and may be selected by pressing $<\mathrm{CR}>$ alone. 


\section{Specifying Integration Time}

The prompt for the integration time of the measurements appears:

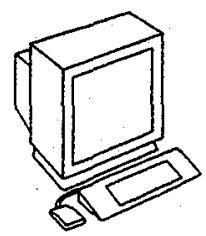

$$
\text { What is the time interval in minutes? [60] }
$$

Because the data you will examine were collected with a 60 -minute integration time, enter:

$$
60<\mathrm{CR}>
$$

\section{Selecting a Site}

QCFIT requests the site identifier of the data you are examining:

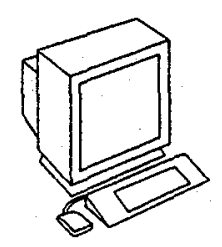

$$
\text { Enter your site identifier: }
$$

You need an identifier of your choice for the Albuquerque site that you are examining. Enter:

$\mathrm{ABQ}<\mathrm{CR}>$

\section{Supplying New Site Data}

Because the identifier you entered is not yet known by QCFIT, the following prompt appears:

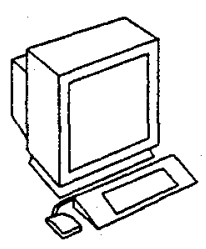

$$
\text { I don't have } A B Q \text {. Is this correct }(Y, N) \text { ? }
$$

Enter:

$$
\mathrm{Y}<\mathrm{CR}>
$$


Because this is a new identifier, QCFIT prompts you for the required information to initialize a QC-zero configuration file for the new site:

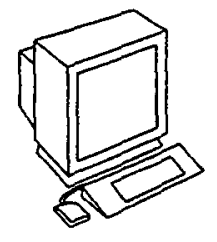

Please give a site description that is not longer than the dashed line:

Enter a short description, such as:

Albuquerque, NM (NOAA) $<\mathrm{CR}>$

QCFIT prompts for geographical information:

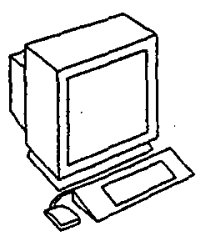

$$
\begin{aligned}
& \text { What is the Latitude in degrees } \\
& \text { (e.g., 40.2693)?: }
\end{aligned}
$$

Enter the latitude for the NOAA site at Albuquerque:

$$
35.05<\mathrm{CR}>
$$

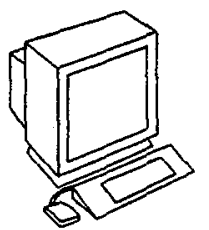

Is this NORTH latitude $(Y, N)$ ? [ $Y$ ]

Enter:

$\mathrm{Y}<\mathrm{CR}>$.

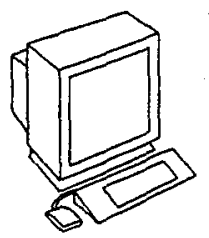

What is the Longitude in degrees (e.g., 95.2711)?

Enter the longitude for the NOAA site at Albuquerque:

$106.62<\mathrm{CR}>$ 


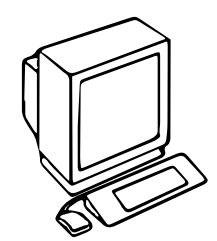

Is this WEST longitude $(Y, N)$ ? [Y]

Enter:

$\mathrm{Y}<\mathrm{CR}>$

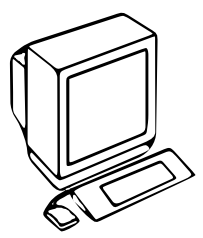

What time zone $(P-8, M-7, C-6, E-5)[-7] ?$

Enter the number that corresponds to mountain standard time, Albuquerque's time zone:

$-7<\mathrm{CR}>$

\section{Specifying the Month}

You must specify the month during which the data to be examined were collected. QCFIT prompts:

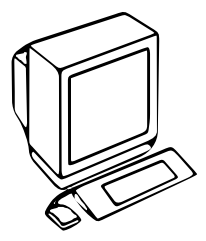

$$
\text { Which month shall be handled (1-12)? }
$$

Enter the month number of June:

$6<\mathrm{CR}>$

\section{Specifying the Data File}

You are prompted for the data file name:

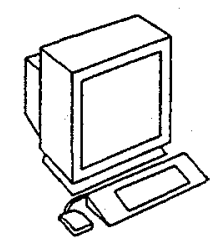

$$
\text { What is the input file name (include extension)? }
$$

Enter the name of the sample data file:

ABQ06A.QCF $<$ CR $>$ 
QCFIT now reads the file, reporting at 100-line intervals as the data are read from the disk:

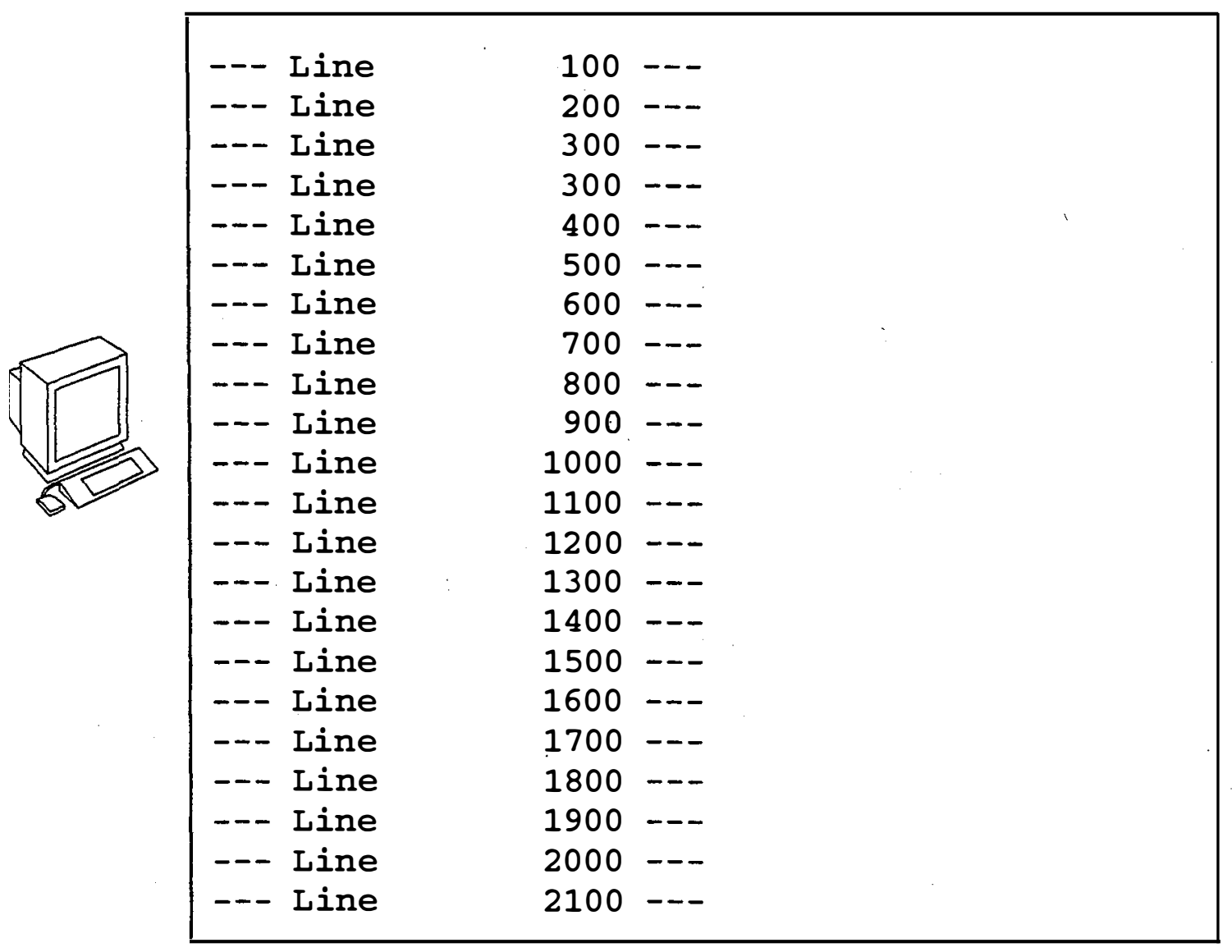

\section{Specifying the Air Mass}

As QCFIT reads the solar irradiance data file, data are segregated into three air mass regimes described as Low, Medium, and High. The Low air mass range is $\leq 1.25$; the Medium air mass range is $>1.25$ and $\leq 2.5$; and the High air mass is $>2.5$ and $\leq 5.58$ (see Chapter 4, Section 4.2.2, page 25, for further discussion).

SERI_QCl makes the same air mass distinction when evaluating solar data; thus, expected values must be determined for each air mass range. Note that no location in the United States has data falling in the Low air mass range for all months.

For each air mass regime for each station-month, SERI_QC requires a left-hand boundary, a right-hand boundary, a maximum $\mathrm{Kt}$ limit, and a maximum $\mathrm{Kn}$ limit (K-values represent the percent possible of extraterrestrial radiation, with $\mathrm{Kt}$ corresponding to the global horizontal value and $\mathrm{Kn}$ corresponding to the direct nomal value). Since this sample session involves only data from June, you need to select only three sets of boundaries, for Low, Medium, and High air masses. 
You are prompted for an initial air mass range:

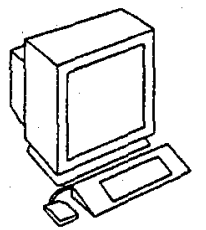

$$
\begin{aligned}
& \text { Which airmass regime, (L) ow, (M)edium, (H)igh? } \\
& {[L]}
\end{aligned}
$$

Specify the low air mass regime:

$\mathrm{L}<\mathrm{CR}>$

QCFIT displays a scatter plot of the data as Kt versus Kn, shown in Figure GS-2, and enters the edit mode.

Briefly, this plot shows you the percent of possible global horizontal along the $\mathrm{X}$-axis $(\mathrm{Kt})$ and the percent of possible direct normal irradiance along the $\mathrm{Y}$-axis $(\mathrm{Kn})$. This site produces a typical scatter plot in which the data generally lie along a lazy-S curve below the diagonal, with a tight clustering of data near the top of the envelope. The data in the cluster represent clear sky irradiance when both global horizontal and direct normal experience the least attenuation. Data farther down in the envelope represent the effects of greater atmospheric attenuation, primarily from clouds.

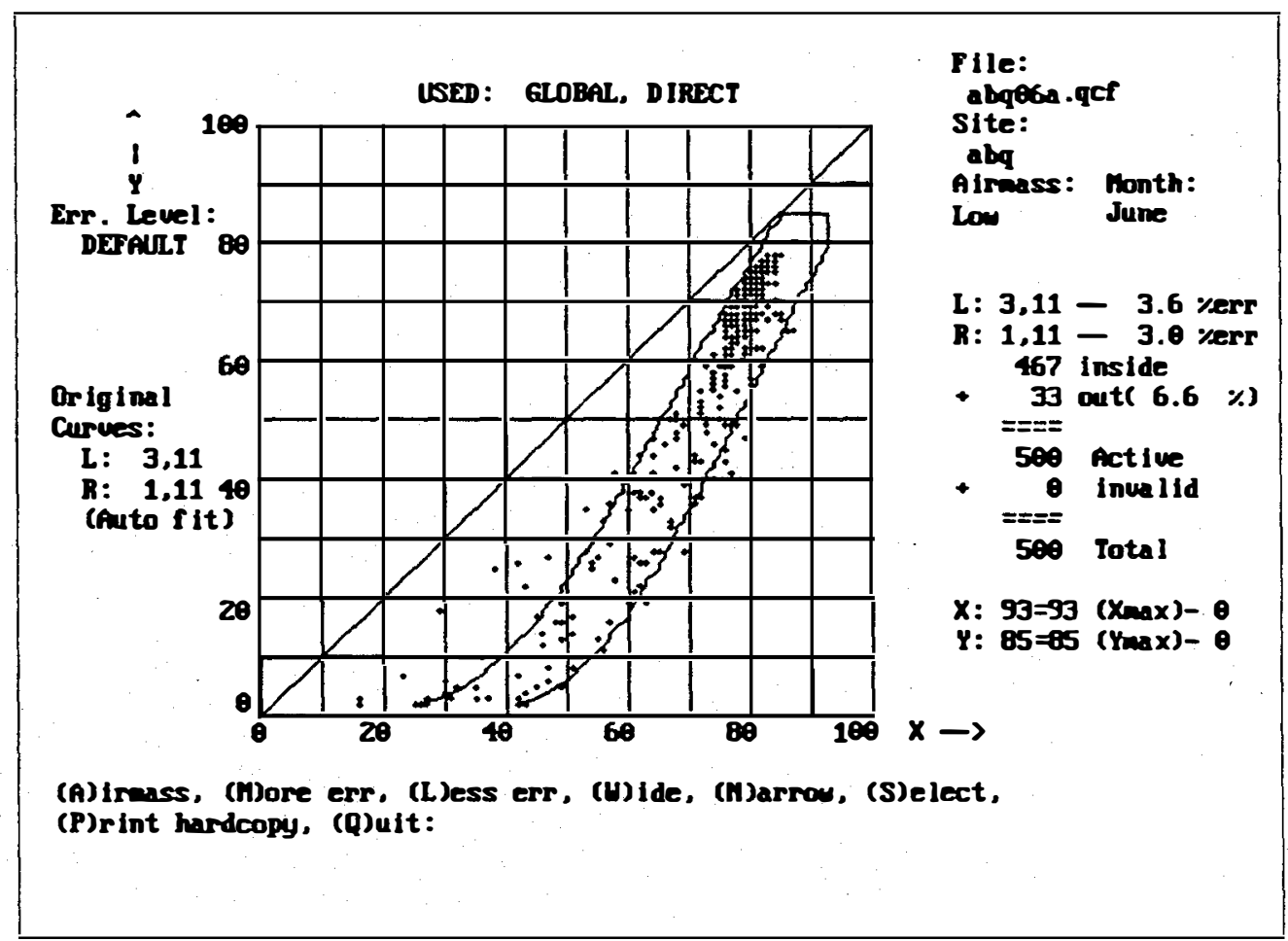

Figure GS-2. Initial Low air mass boundary fit by QCFIT 
Your goal is to superimpose the solid line boundaries on the scatter plot to represent an area where data from this site are most likely to fall, or conversely, define an area outside the general scatter where data are suspect. Using the QCFIT boundary editing tools, you can change the shape and position of the right or left boundaries to achieve an envelope that encompasses most of the data.

On your screen and in Figure GS-2, you can see that QCFIT made an initial attempt at fitting the data, as evidenced by the "Auto fit" note under the "Original Curves" label on the left side of the screen. In the same part of the screen, the curves are identified as "L: 3,11" and "R: 1,11." This indicates that the left boundary is shape 3, position 11, and the right boundary is shape 1, position 11 . (All the shapes are shown in Chapter 5, Figures 5-2, 5-3, and 5-4, pages 36 and 37.)

On the right side of the screen, you can see the boundaries identified again, this time followed by error statistics: $3.6 \%$ on the left and $3.0 \%$ on the right. These values indicate the percentage of all data points that lie outside the boundaries to the left and right, respectively. By adjusting the curves you hope to find a balance between good data density inside the boundaries and a low percentage of data outside the boundaries, typically between $1 \%$ and $5 \%$.

An examination of the scatter plot shows that an adjustment of the curves could include more data without having unwanted blank areas inside the envelope. First, you can make QCFIT attempt another curve fitting, this time excluding fewer points. From the menu at the bottom of the screen, select the (L)ess err option by entering:

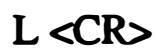

QCFIT redraws the screen, as shown in Figure GS-3. 


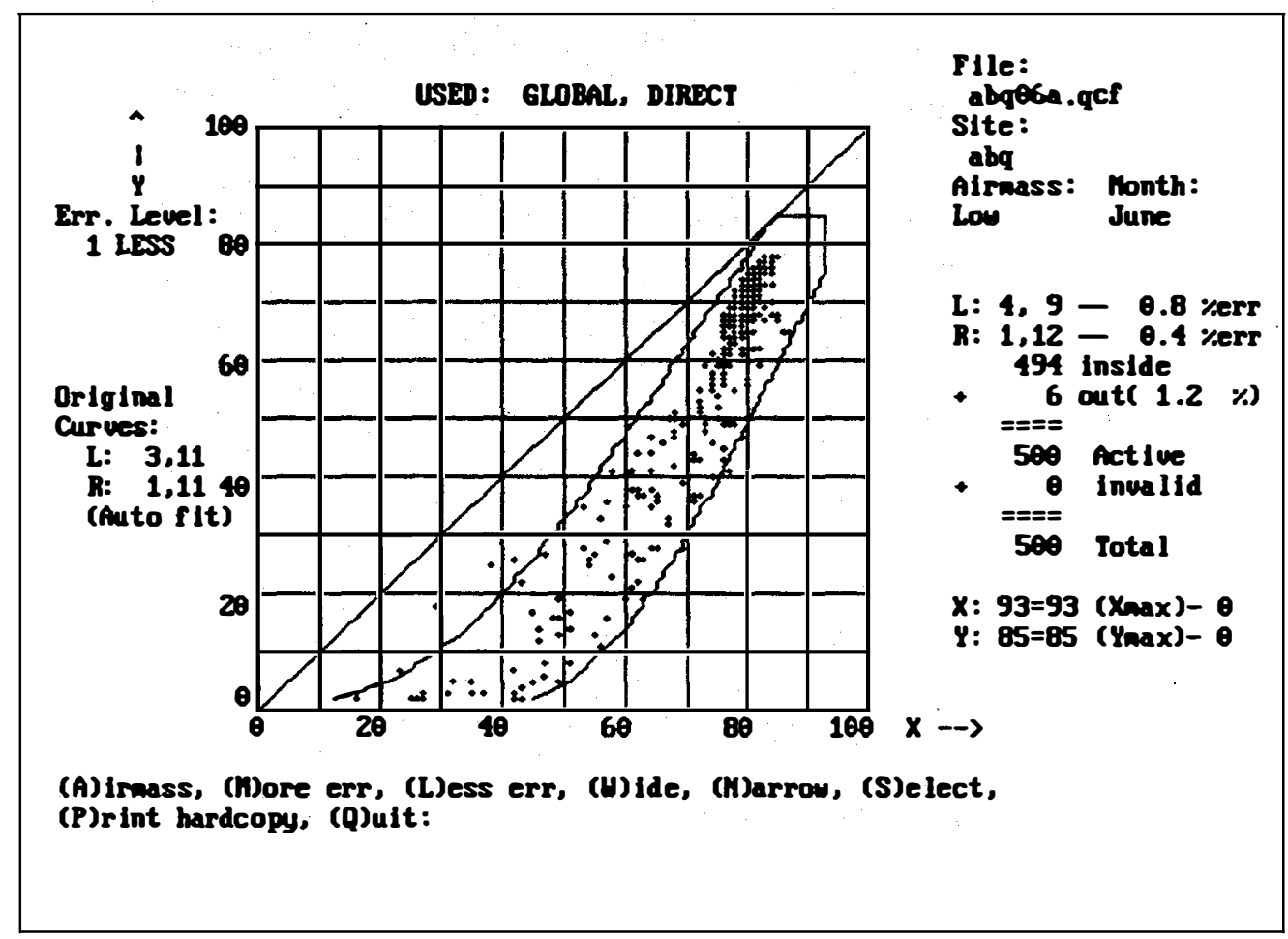

Figure GS-3. Making wider boundaries with the (L)ess err option

Now you can see that QCFIT has chosen a left curve with a shallower slope and has positioned it more to the left. For the right curve, QCFIT stayed with the same shape, but moved it slightly to the right. The left boundary is troublesome, however, because of the gap to the left of the dense cluster of data near the top (for now ignore the blank area inside the boundaries to the right and above the scatter plot). By narrowing the envelope slightly, a truer fit is possible. Select (N)arrow from the editing menu options by entering:

$$
\mathrm{N}<\mathrm{CR}>
$$

QCFIT then prompts:

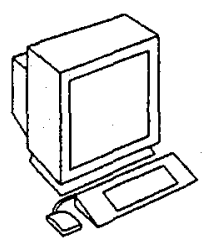

$$
\text { The LEFT side? [Y] }
$$

Because you want to move the left boundary toward the middle of the envelope, enter:

$$
\mathrm{Y}<\mathrm{CR}>\text {. }
$$


QCFIT then prompts:

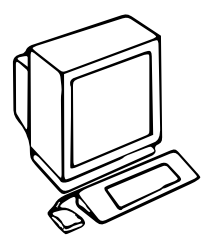

The RIGHT side? [Y]

Because the right curve is satisfactory for the moment, enter:

$\mathbf{N}<\mathrm{CR}>$

QCFIT then moves the left boundary toward the center of the scatter plot as shown in Figure GS-4.

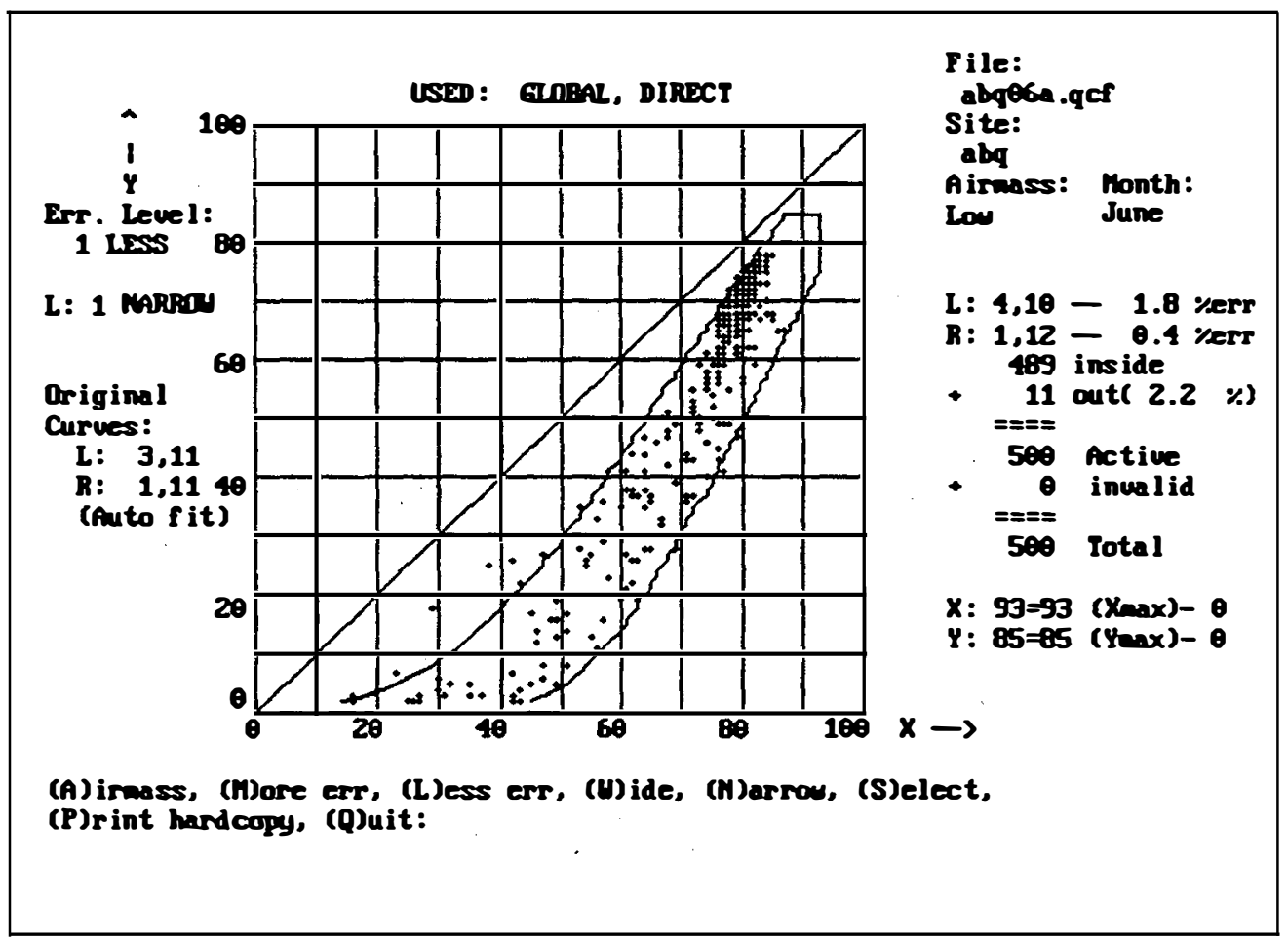

Figure GS-4. Moving the left boundary with (N)arrow

Now both the left and right boundaries follow, almost in dot-to-dot fashion, the data points that define the envelope. However, you must also consider data density when setting boundaries. Notice how the right curve in Figure GS-4 lies some distance from the dense cluster of points near the top of the envelope. A curve with a steeper slope should produce a better fit to the dense part of the scatter plot, even though it may exclude a few outliers.

Further, the large blank area, to the right and above the scatter of data, mentioned previously, must be removed by specifying maximum K-values. Both of these faults may be remedied with the (S)elect option by entering:

$$
\mathrm{S}<\mathrm{CR}>
$$


This option allows you to select specific curves and maximum K-values. QCFIT asks if you wish to specify a left boundary curve:

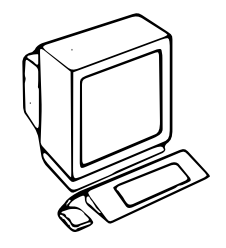

$$
\text { The LEFT side? [Y] }
$$

Because you do not want to change the left curve, enter:

$$
\mathrm{N}<\mathrm{CR}>
$$

QCFIT then asks if you want to change the right curve:

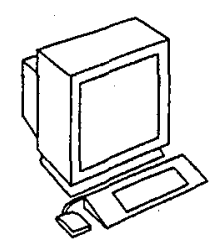

$$
\text { The RIGHT side? [Y] }
$$

To specify a new right boundary, enter:

$\mathrm{Y}<\mathrm{CR}>$

QCFIT asks for the curve type and position:

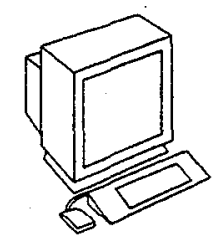

$$
\text { Please enter the curve type and position: }[1,12]
$$

The pair of numbers in brackets identify the current curve and its position. The left number is the curve number from Figures 5-2 or 5-3, Chapter 5, (page 36) and the right number is its horizontal position, as shown in Figure 5-4. From Figure 5-3, you see that curve 2 has a steeper slope than curve 1 , so you will specify curve 2 , position 11. Enter:

$$
2,11<\mathrm{CR}>
$$

In the Menu Box you now see:

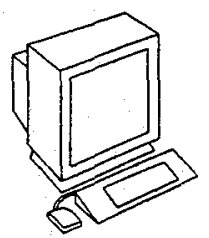

$$
X \max [Y]
$$

To specify a Kt maximum (along the $\mathrm{X}$-axis), enter:

$$
\mathrm{Y}<\mathrm{CR}>
$$


You see this prompt:

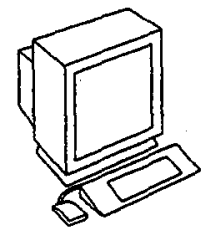

$$
\text { What value should it be? [93] }
$$

By examining the scatter plot, you can see that, with minor exceptions, all of the data fall below 85 on the $\mathrm{X}$-axis. Therefore, enter:

\section{$85<\mathrm{CR}>$}

QCFIT then asks if you want to specify a maximum for the Y-axis:

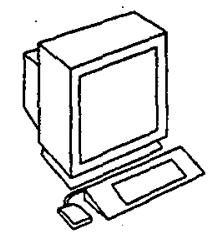

$$
Y \max [Y]
$$

To specify a Kn maximum (along the Y-axis), enter:

$$
\mathrm{Y}<\mathrm{CR}>
$$

You see this prompt:

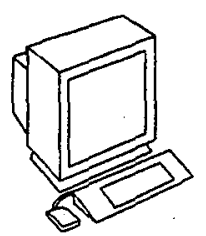

$$
\text { What value should it be? [85] }
$$

By examining the scatter plot, you can see that all of the data fall below 78 on the Y-axis. Therefore, enter:

\section{$78<\mathrm{CR}>$}

QCFIT redraws the scatter plot with the new maximums as part of the boundaries, as shown in Figure GS-5. 


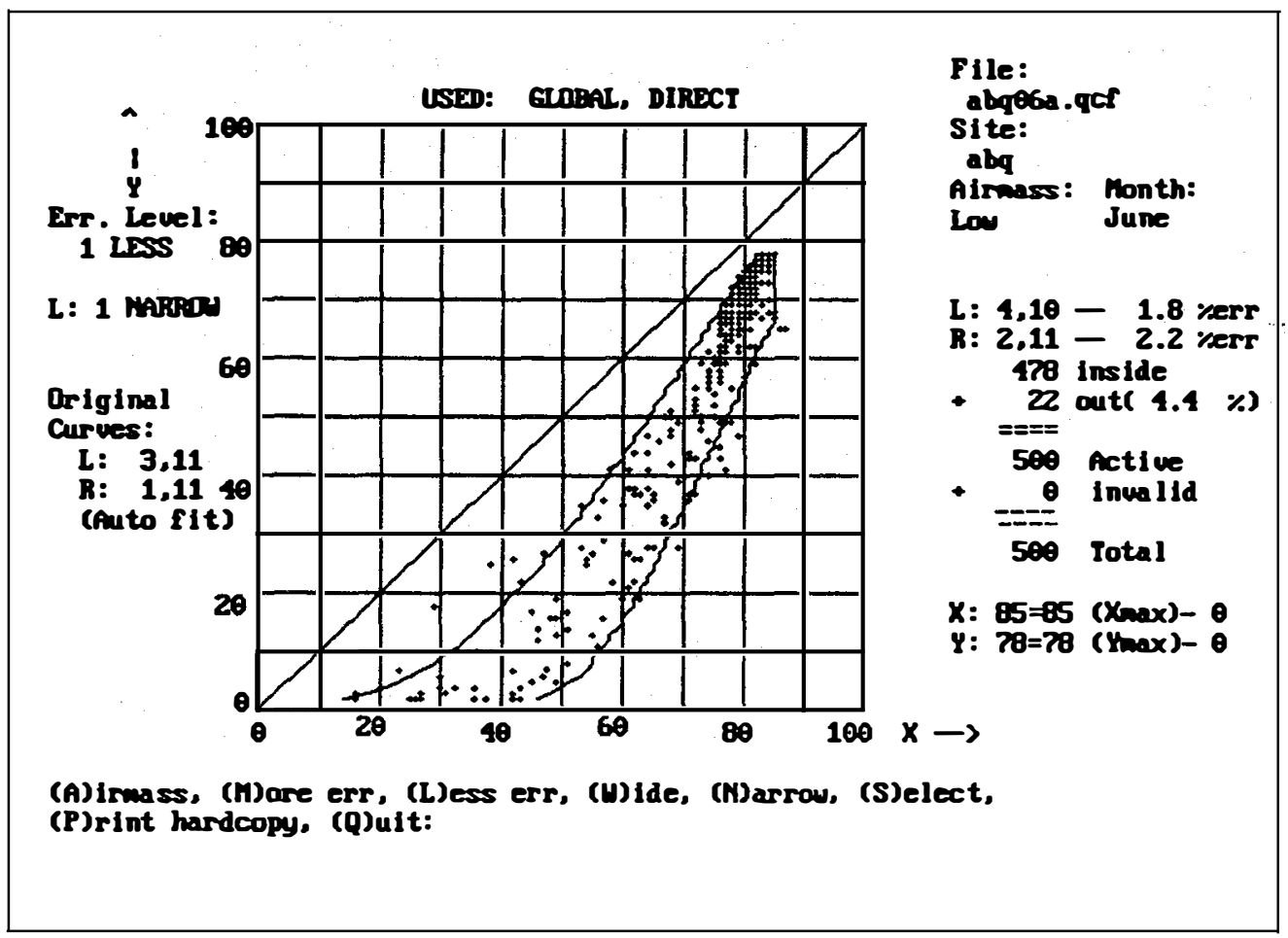

Figure GS-5. Setting the right curve and $\mathrm{K}$ maximums with (S)elect

Note that the new maximums are shown in the lower right-hand comer of the screen. See the "X/Y Max Box" explanation of this information in Chapter 8, Section 8.4.1 (page 117). Note also under "Original curves," that the first curves attempted by QCFIT remain displayed for reference.

In Figure GS-5, both the left and right boundaries enclose nearly all of the data points, and they closely follow the general outline of the data. A few anomalous data points lie outside the boundaries, which is desired either because they are bad data, or because to include them would expand the envelope to regions where data seldom or never occur. Note the error statistics on the right side of the screen: $1.8 \%$ and $2.2 \%$ for the left and right boundaries, respectively. These numbers are at the lower end of the suggested $1 \%$ to $5 \%$ range but can be justified by the welldefined scatter plot and quality of the boundary fit.

Compare the edited boundaries in Figure GS- 5 with the auto-fit boundaries in Figure GS-2. Notice how the right and left boundaries in Figure GS-2 nearly parallel each other, whereas the envelope in Figure GS-5 has the more typical shape that narrows toward the top. Be aware that the automatic curve fitting algorithm in QCFIT works well in dense areas of the scatter plot but may choose inappropriate curves in areas of sparse data. 
Now that you are satisfied with the boundaries for the low air mass data, examine data for the medium air mass. From the Editing menu, select (A)ir mass by entering:

\section{$\mathrm{A}<\mathrm{CR}>$}

Before considering data from another air mass regime, QCFIT confirms the right and left boundary specifications for the current air mass regime:

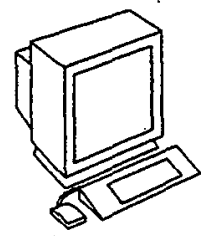

Okay to update file with $\mathrm{R}: 4,10$; L: 2, 11 $(Y, N) ?[Y]$

To save the boundaries specified for the Low air mass, enter:

$\mathrm{Y}<\mathrm{CR}>$

QCFIT prompts for the new air mass specification:

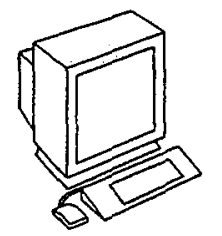

$$
\text { Which air mass regime, (L) ow, (M) edium, (H) igh? }
$$
[L]

Specify the Medium air mass regime by entering:

$\mathrm{M}<\mathrm{CR}>$

QCFIT now plots the medium air mass data and makes an initial fit, shown in Figure GS-6. 


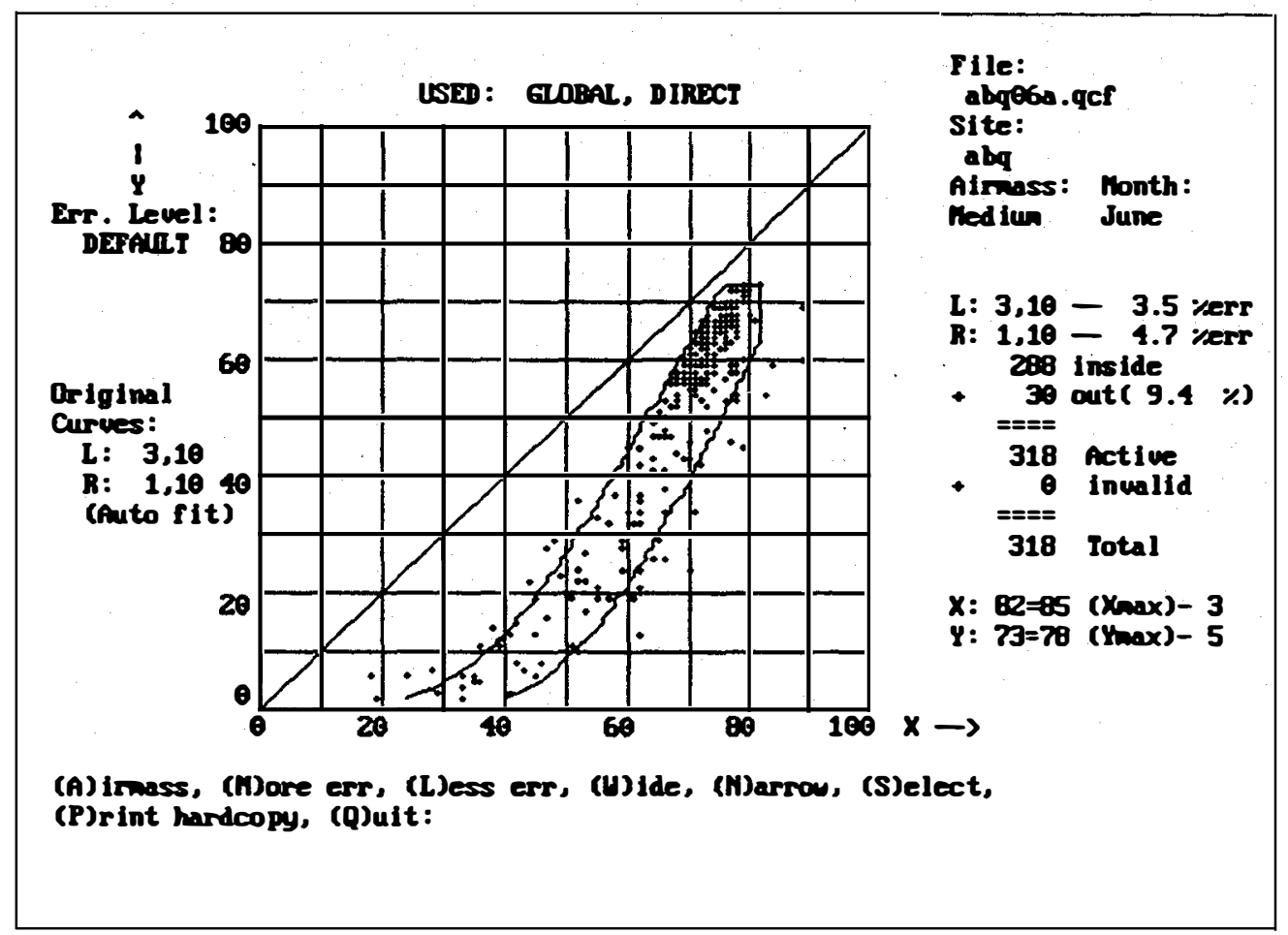

Figure GS-6. Initial Medium air mass curve placement

Again, although the error statistics are reasonable, it looks as if some adjustment in the curves could produce a better fit. As before, use the (S)elect editing option to specify a shallower slope for the left curve $(4,9)$ and a steeper slope for the right curve $(2,10)$ :

\section{$\mathrm{S}<\mathrm{CR}>$}

As noted previously when editing the Low air mass, this option allows you to select either specific curves or select maximum $\mathrm{K}$ values.

QCFIT asks if you wish to specify a left boundary curve:

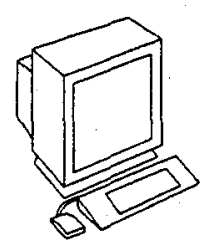

$$
\text { The LEFT side? [Y] }
$$

Because you want to specify a different left curve, enter

$$
\mathrm{Y}<\mathrm{CR}>
$$


QCFIT asks for the curve type and position:

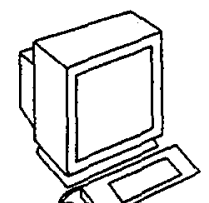

$$
\text { Please enter the curve type and position: }[3,10]
$$

Specify curve 4, position 9:

$4,9<\mathrm{CR}>$

QCFIT then asks if you wish to specify a right boundary.

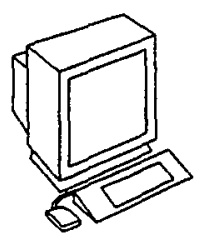

$$
\text { The RIGHT side? [Y] }
$$

\section{Enter:}

\section{$\mathrm{Y}<\mathrm{CR}>$}

QCFIT asks for the curve type and position:

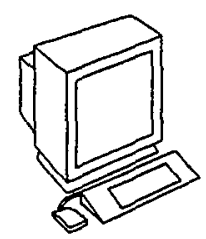

$$
\text { Please enter the curve type and position: }[1,10]
$$

Specify curve 2, position 10 :

$2,10<C R>$

In the Menu Box you now see:

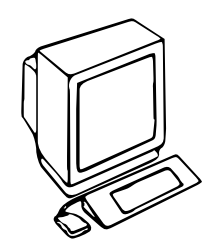

$X \max ?[Y]$

Because you do not want to specify a Kt maximum, enter:

$\mathrm{N}<\mathrm{CR}>$ 
QCFIT then asks if you want to specify a maximum for the Y-axis

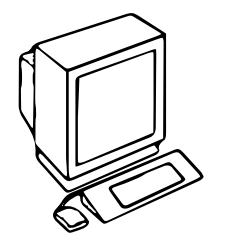

$Y \max ?[Y]$

Again, you do not want to specify a maximum, so enter:

$\mathrm{N}<\mathrm{CR}>$

Your screen now looks like Figure GS-7.

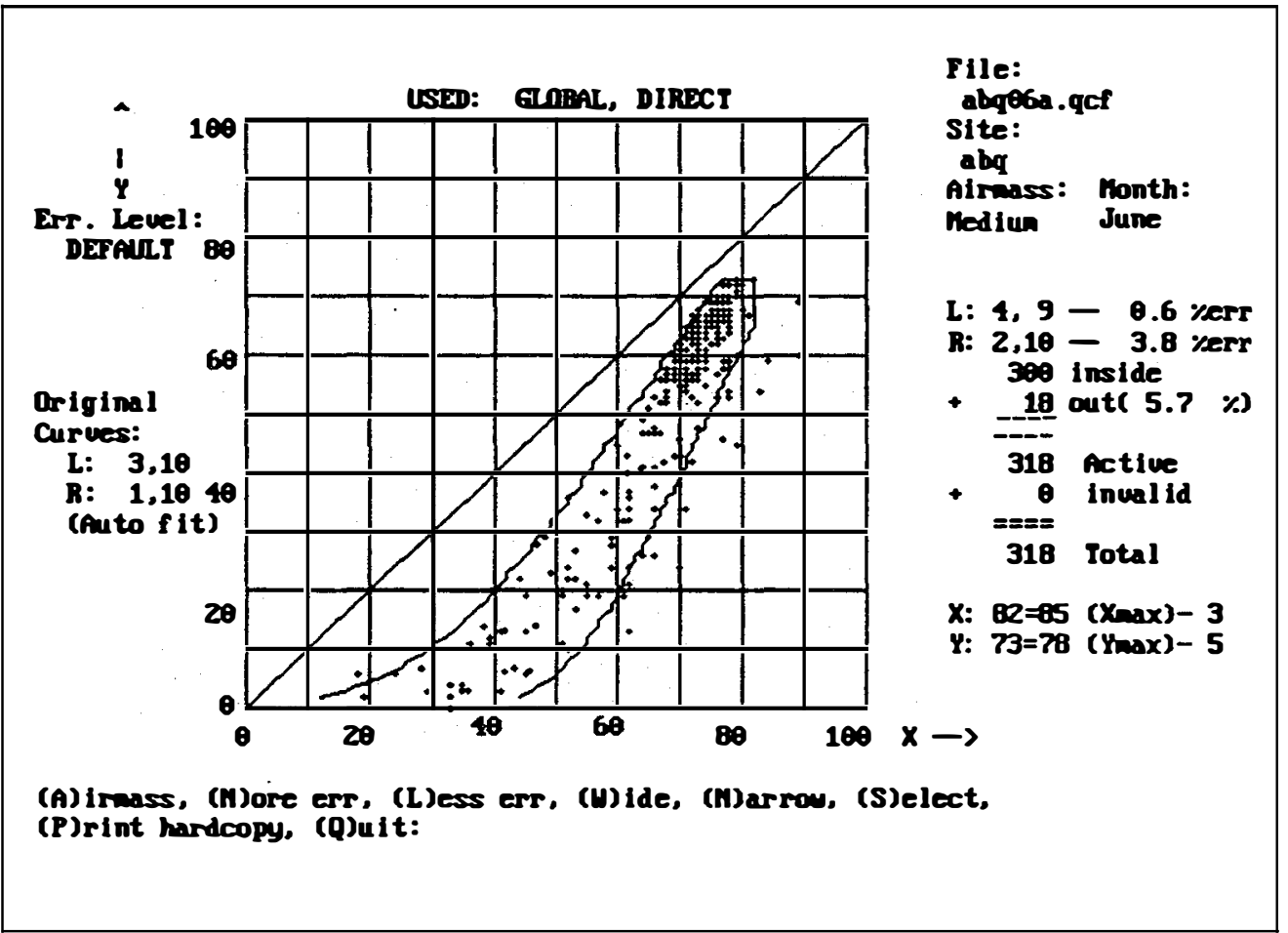

Figure GS-7. Medium air mass after adjusting curves

Note in the lower right corner of the display that the $\mathrm{X}$ and $\mathrm{Y}$ maximums show lower numbers than the 85 and 78 that you specified while examining data for the Low air mass. This is because QCFIT uses only one set of maximum specifications for all air mass regimes and automatically adjusts those specifications to lower values for higher air mass regimes. Changing either maximum value for one air mass regime also changes it for the other two regimes.

Because these boundaries for the Medium air mass regime look good, use the (A)irmass editing option described previously to select the High air mass regime. QCFIT produces the screen shown in Figure GS-8. 


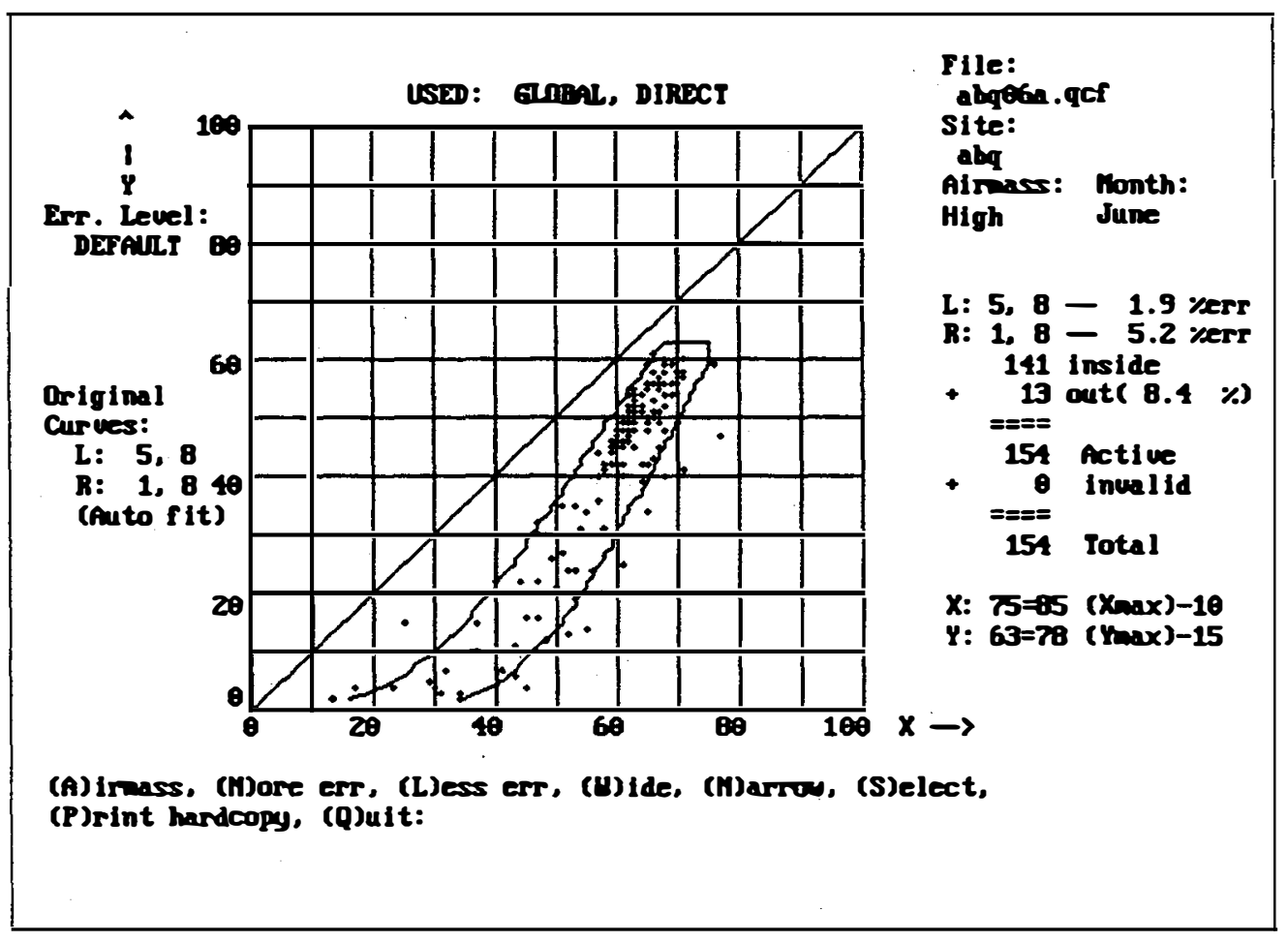

Figure GS-8. Initial curve placement for High air mass

Here the left boundary looks like a good fit, but the right boundary could be adjusted to accommodate some of the lower $\mathrm{Kn}$ data points. For this case, specify a curve with a steeper slope than curve 1 . Choose the (S)elect editing option by entering:

\section{$\mathrm{S}<\mathrm{CR}>$}

QCFIT asks if you wish to specify a left boundary curve:

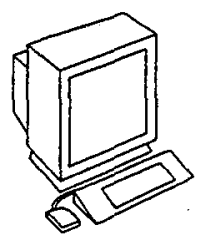

$$
\text { The LEFT side? [Y] }
$$

Because the left boundary is okay, enter:

$\mathrm{N}<\mathrm{CR}>$ 
QCFIT then asks if you want to change the right curve:

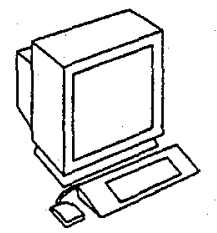

$$
\text { The RIGHT side? [Y] }
$$

To specify a new right boundary, enter:

$\mathrm{Y}<\mathrm{CR}>$

QCFIT asks for the curve type and position.

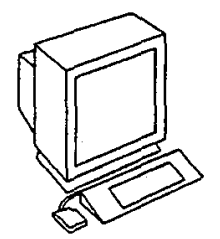

$$
\text { Please enter the curve type and position: [1,8] }
$$

From Figure 5-3, Chapter 5 (page 36), you see that curve 2 has a steeper slope than curve 1 , so specify curve 2 , position 8 . Enter:

$$
2,8<\mathrm{CR}>
$$

In the Menu Box you now see:

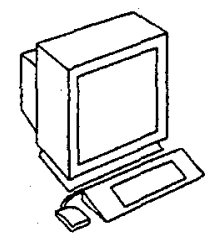

$$
X \max [Y]
$$

Because you do not want to specify a Kt maximum, enter:

$\mathrm{N}<\mathrm{CR}>$

QCFIT then asks if you want to specify a maximum for the Y-axis:

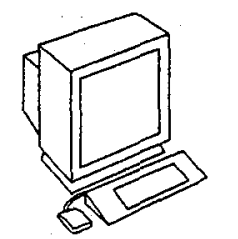

$$
Y \max [Y]
$$

Again, you do not want to specify a Kn maximum, so enter:

$$
\mathrm{N}<\mathrm{CR}>
$$

QCFIT redraws the scatter plot with the new right boundary, as shown in Figure GS-9. 


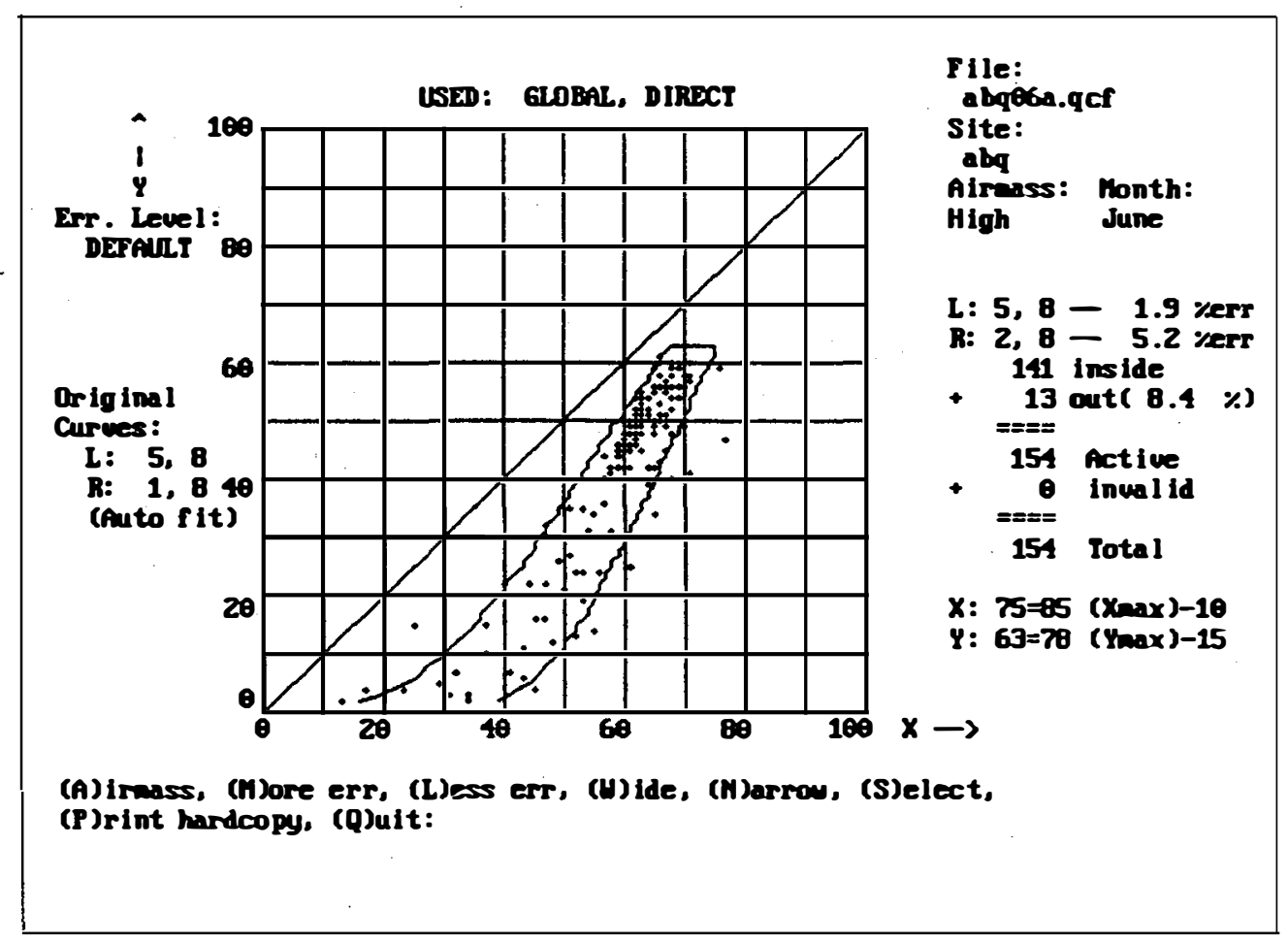

Figure GS-9. New right curve after using (S)elect

This scatter plot indicates that an even steeper curve could be used for the right boundary. Repeat the preceding (S)elect procedure and specify curve 3,8 for the right boundary without changing the left boundary or the $\mathrm{K}$ maximum values. The resulting scatter plot is shown in Figure GS-10. 


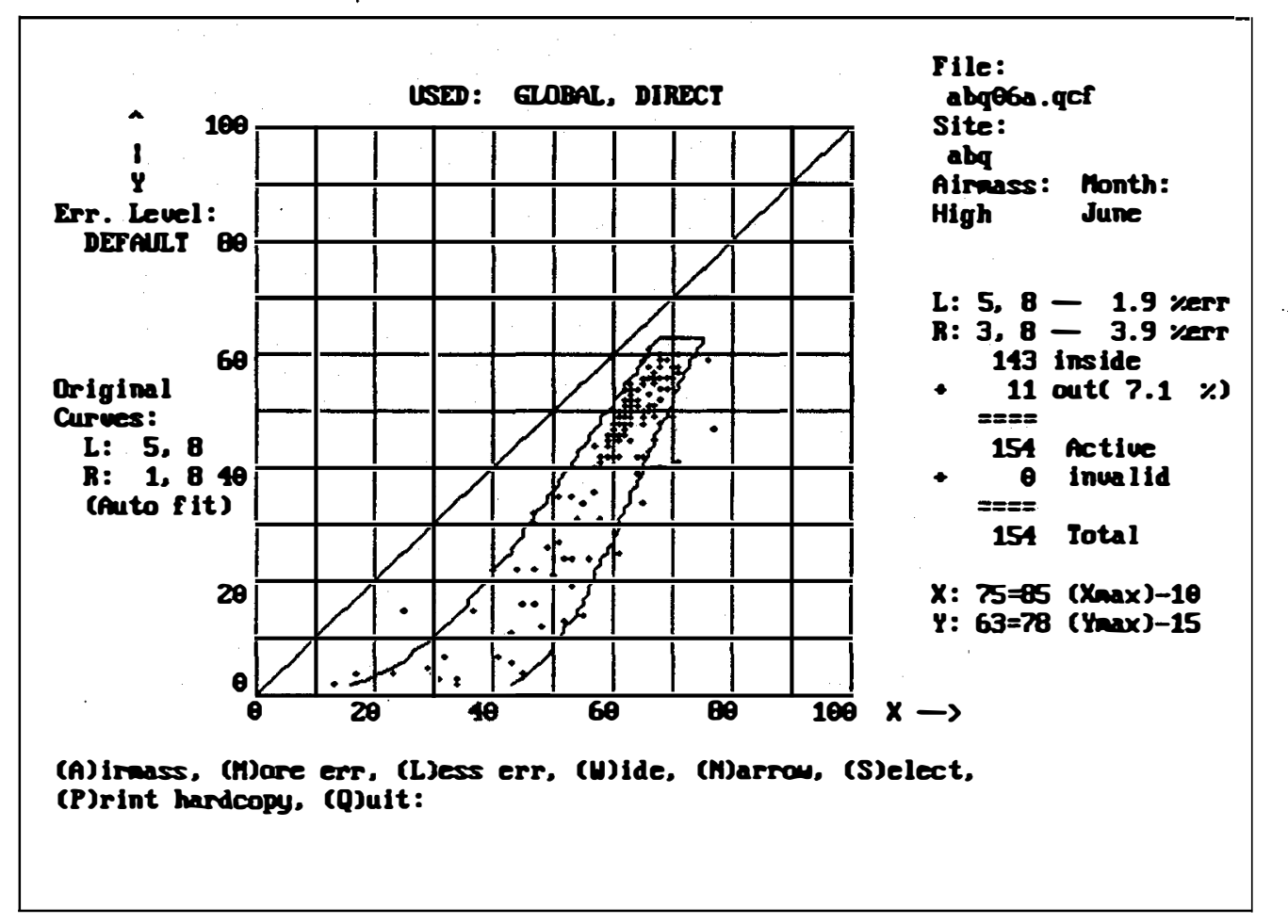

Figure GS-10. A steeper right curve using (S)elect

With these final boundary specifications, you are done with the June data and can exit the program. Select (Q)uit from the Editing menu:

$$
\mathrm{Q}<\mathrm{CR}>
$$

QCFIT responds with the confirmation prompt for the curves just specified in the High air mass regime:

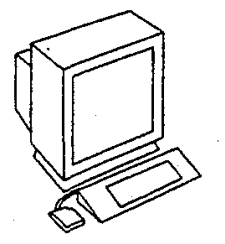

$$
\begin{aligned}
& \text { OK to update file with } R: 3,8 ; \mathrm{L}: 5,8(\mathrm{Y}, \mathrm{N}) \text { ? } \\
& {[\mathrm{Y}]}
\end{aligned}
$$

Enter:

$\mathrm{Y}<\mathrm{CR}>$ 
QCFIT prompts for confirmation of $\mathrm{Kt}_{\max }$ and $\mathrm{Kn}_{\max }$ :

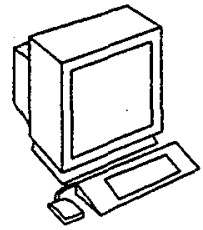

OK to update file with $\operatorname{Max} \mathrm{KT}=0.85$,

$\operatorname{Max} \mathrm{KN}=0.78(\mathrm{Y}, \mathrm{N}) \quad[\mathrm{Y}]$

The values displayed are the actual $\mathrm{K}$ values translated from the $\mathrm{Xmax}$ and $\mathrm{Ymax}$ integers you entered earlier. Remember, the displayed max values are for the low air mass data; the values for the other air mass regimes are calculated. Enter:

\section{$\mathrm{Y}<\mathrm{CR}>$}

QCFIT prompts for continued operation:

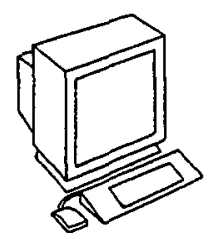

$$
\text { Do you wish to process another file? [Y] }
$$

Normally, you would proceed to the next month's data, but your sample session is done, so enter:

$\mathrm{N}<\mathrm{CR}>$ 


\section{Examining Results from the Session}

A new file should reside in the current directory called S_ABQ.QC0. This file, known as a site's $Q C$-zero file, contains the expected value information created by the QCFIT session just concluded, and is used by the SERI_QC1 subroutine when evaluating solar measurements for a site.

Using the DOS TYPE command, display the file on the screen:

TYPE S_ABQ.QC0 <CR $>$

You should see the following data on the screen:

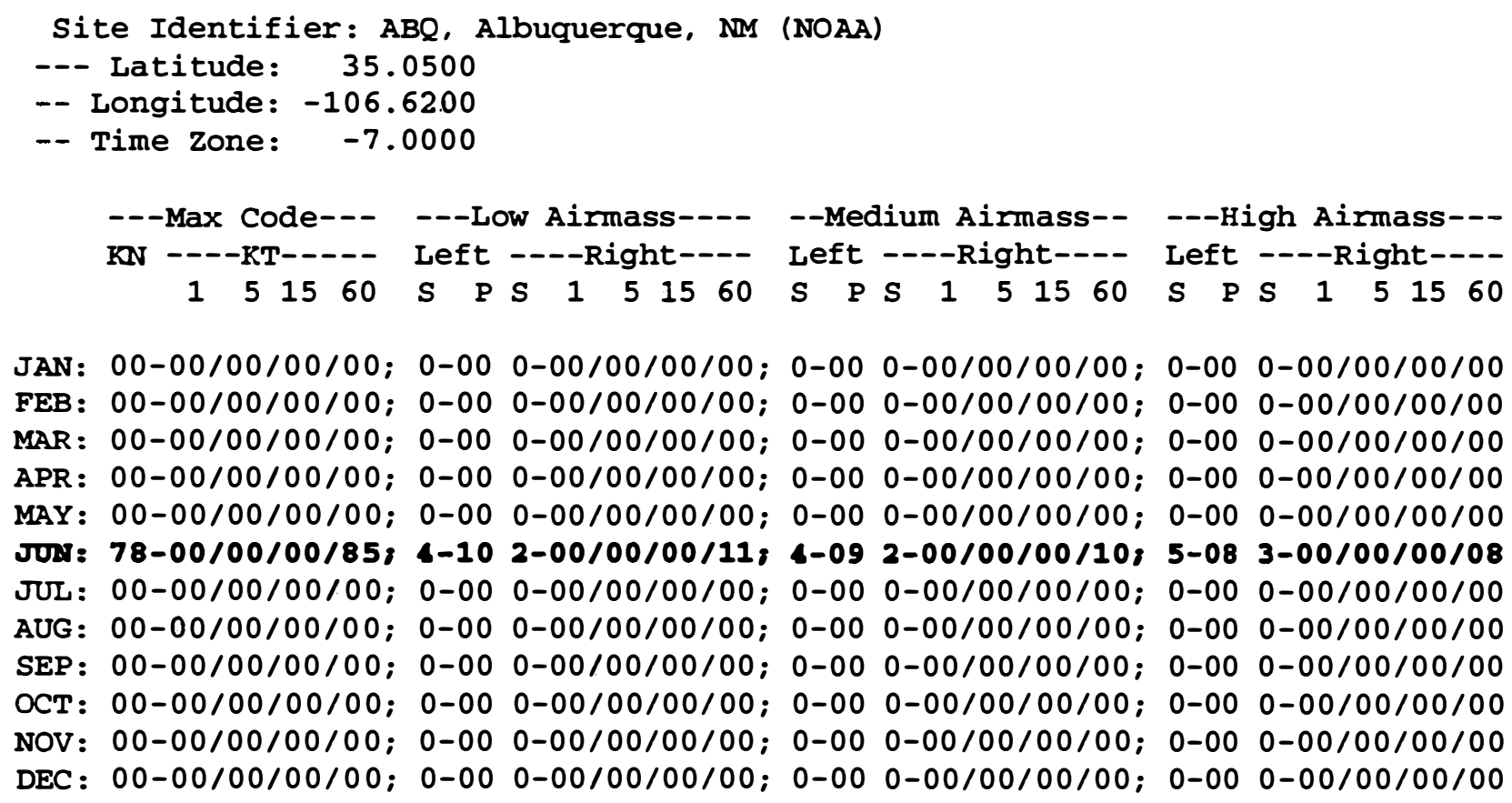

The information contained in this file is detailed in Chapter 7, Section 7.3 (page 87), and is generally transparent to the user. We examine it here, however, to emphasize the fact that this file is created or modified by QCFIT, as exemplified by the highlighted line for June.

Examining the QC-zero file is also a valuable tool to detect seasonal trends in your data and to spot anomalous curve selections due to small data sets or aberrant measurements. Although the curve selection in this sample session has produced acceptable curves, you may wish to examine file S_23050.QC0, included in the FILES.QC0 subdirectory on the SERI QC distribution disk. This file, produced from the same Albuquerque data, shows different curve selections. The curves were edited based partly on the characteristics of data from adjacent months. Any attempt at this type of curve editing should be based on a thorough knowledge of your site's climate. 
These differences between the sample session and the QC-zero file on the distribution disk also underscore the subjective nature of boundary fitting. Different individuals working with the same data will very likely develop two different sets of boundaries, both of which may be equally acceptable. Even a particular individual may choose a different set of boundaries if working with the same data set at different times. The most important aspect of choosing boundaries is that they closely represent the general scatter of data. 


\section{Using the SERI_QC1 Subroutine}

Chapter 9 (page 145), SERI_QC1 Software Users Guide, explains how to call the SERI_QC1 subroutine from your own programs. A sample program, QA_ABQ.FOR, is included on the distribution disk, and its use is briefly explained here. Note that program QA_ABQ calls SERI_QC1.

You must first compile the SERI_QC1.FOR subroutine and the QA_ABQ.FOR sample program. On our system, we use the following FORTRAN compiler commands. Your system may require different commands.

F77L QA_ABQ $<$ CR>

F77L SERI_QC1 $<$ CR $>$

Then the two object codes must be linked into an executable program. On our system, the LINK command is:

$$
\text { OPTLINK QA_ABQ+SERI_QC1,QA_ABQ,, }
$$

This produces an executable file called QA_ABQ.EXE. Run this program by entering:

$$
\text { QA_ABQ }<\text { CR }>
$$

The program asks for an input file name:

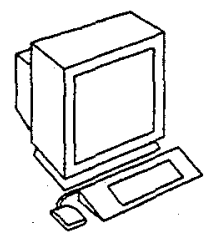

$$
\text { Enter the input file name: }
$$

Enter the name of the 88-90 sample data set for the Albuquerque NOAA site:

\section{ABQ06C.QCF <CR>}

As the program runs, it reads the solar irradiance file ABQ06C.QCF and accumulates the occurrences of quality assessment flags based on the boundary and expected value information you created earlier in the sample session. The program creates an output file called QA_ABQ.OUT. 
Type or print QA_ABQ.OUT. You should see the following listing:

\begin{tabular}{rrrr} 
& \multicolumn{3}{c}{ ABQ06C.QCF } \\
Flag & $\begin{array}{r}\text { IQCglo } \\
\text { Count }\end{array}$ & $\begin{array}{r}\text { IQCdir } \\
\text { Count }\end{array}$ & $\begin{array}{r}\text { IQCdif } \\
\text { Count }\end{array}$ \\
1 & 609 & 599 & 0 \\
2 & 577 & 577 & 0 \\
8 & 0 & 10 & 0 \\
12 & 2 & 1 & 0 \\
13 & 1 & 2 & 0 \\
16 & 1 & 1 & 0 \\
17 & 1 & 1 & 0 \\
24 & 2 & 0 & 0 \\
25 & 0 & 2 & 0 \\
32 & 6 & 0 & 0 \\
33 & 0 & 6 & 0 \\
99 & 0 & 0 & 1199
\end{tabular}

You can also run the program on the sample file ABQ06B.QCF, which contains data from the Albuquerque NOAA site from 1981 to 1985. The data collected during the 1981 to 1985 period suffers from various quality problems, and they produce a QA_ABQ.OUT file with a significantly different distribution of flags.

The program QA_ABQ.FOR is explained and annotated in Chapter 9, Section 9.2 (page 148). The quality assessment flags shown in this report are detailed in Chapter 6, Section 6.4. (page 46) and Table 6-2 (page 47).

The files in the FILES.QC0 subdirectory on the distribution disk are QC-zero files developed by NREL from NOAA network data collected between 1978 and 1980. They may be used as a starting point for sites with similar geographical and climate characteristics, and for sites where no historical data exist. 



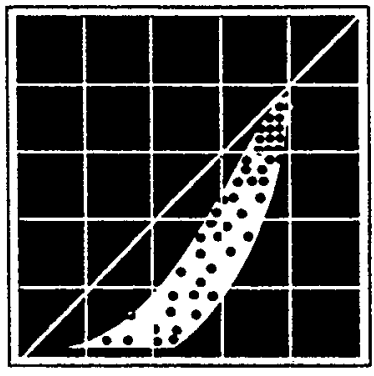

\section{Chapter 7 \\ The SERI QC Software and Hardware Environment}

\section{Contents}

7.1 Hardware Requirements .................................................... 84

7.1.1 VAX Version ........................................................... 84

7.1.2 PC Version ............................................................... 84

$7.2 \quad$ Data Requirements ............................................................ 86

7.3 QC-zero Configuration Files ............................................... 87

$7.4 \quad$ SOLPOS Subroutine ........................................................... 91

$7.5 \quad$ Gompertz Curves ............................................................... 95

7.5.1 Defining Gompertz Curves ........................................ 95

7.5.2 Storing Gompertz Data .............................................. 95

\section{Overview}

This chapter provides background information on the hardware requirements and common elements of the SERI_QC1 and QCFIT software included in the SERI QC package. Chapters 8 and 9 contain instructions for running the software. 


\subsection{Hardware Requirements}

The SERL_QC1 subroutine and QCFIT program were developed on a VAX using the VMS operating system and a VAX FORTRAN-77 compiler. The programs were subsequently ported to the IBM PC.

\subsubsection{VAX Version}

The VAX version of the SERI_QC1 subroutine was written in VAX FORTRAN and contains some compiler-specific functions that may not be portable. If you wish to port the SERI_QC1 subroutine to another compiler, use the PC version described in the following section.

The QCFIT program also contains several VMS-specific routines that make it incompatible with other systems. To run QCFIT, terminals, such as the DEC VT$24 x$ series, must be capable of interpreting ReGIS graphics. The Print Screen command in QCFIT requires a DEC LA100 or compatible printer attached to the user's terminal printer output port. If no printer is attached, the program ignores the Print Screen command but otherwise runs normally.

Memory and disk requirements for the VAX version (including source, object, and executable files) are specified in Table 7-1.

Table 7-1. VAX Memory and Disk Requirements

\begin{tabular}{|l|c|c|}
\hline & Disk Space & $\begin{array}{c}\text { Memory } \\
\text { Required }\end{array}$ \\
\hline \hline Subroutine SERI_QC1 & $65 \mathrm{kB}$ & $25 \mathrm{kB}$ \\
\hline Program QCFIT & $100 \mathrm{kB}$ & $250 \mathrm{kB}$ \\
\hline
\end{tabular}

Other disk requirements: Approximately 2 kilobytes per QC-zero file (required for each measurement station) and the space necessary for the solar radiation data files to be processed by QCFIT and/or SERI_QC1.

\subsubsection{PC Version}

The PC version of the SERI_QC1 subroutine should be portable to any FORTRAN-77 compiler that supports the following language extensions:

- Variable names may be longer than 6 characters

- Variable names may include the underscore character (“"_)

- Variables may be initialized with slash characters (" $\Gamma$ ') when declared

- Integer*2 data type. 
The object file distributed with the SERI QC package was compiled with the Lahey Computer Systems, Inc. F77L Version 4 FORTRAN compiler. NREL does not provide support for linking the distributed object file to the object code generated with other compilers.

The PC version of QCFIT relies heavily on the Lahey FORTRAN Graphoria graphics library and is not portable to other compilers. The executable (run-time) code distributed with the SERI QC package was compiled with the Lahey F77L Version 4 FORTRAN compiler.

The software requires an IBM-compatible PC, XT, AT, or Intel-based 8088, 8086, 80286,80386 or 80486 computer, a math co-processor, and the MS-DOS operating system. In addition, QCCFIT requires a VGA color graphics monitor. Hard copy output requires one of the following:

- $\quad$ PostScript printer

- $\quad$ Hewlett-Packard LaserJet III or LaserJet 4 printer

- MS-DOS 4.0 or 5.0 and a graphics printer compatible with the MS-DOS GRAPHICS command.

If you want hard copy output of your QCFIT scatter plots, the printer drivers distributed with the QCFIT package (HPLJ.BIN and EPS-P.BIN) must reside in the directory from which QCFIT is run.

Memory and disk requirements for the PC version (including sources, object, and executable files) are specified in Table 7-2.

Table 7-2. PC Memory and Disk Requirements

\begin{tabular}{|l|c|c|}
\hline & Disk Space & $\begin{array}{c}\text { Memory } \\
\text { Required }\end{array}$ \\
\hline \hline Subroutine SERI_QCl & $30 \mathrm{kB}$ & $25 \mathrm{kB}$ \\
\hline Program QCFIT & $220 \mathrm{kB}$ & $500 \mathrm{kB}$ \\
\hline
\end{tabular}

Other disk requirements: Approximately 2 kilobytes per QC-zero file (one required for each measurement station) and the space necessary for the solar radiation data files to be processed by QCFIT and/or SERI_QC1.

NREL has tested the software on a variety of hardware configurations but does not guarantee that it will run on equipment meeting the above specifications. 


\subsection{Data Requirements}

The solar irradiance input data must be in watts per square meter $\left(\mathrm{W} / \mathrm{m}^{2}\right)$ for the SERI_QC1 subroutine. If your data are in different units, your calling program must make the conversion to $\mathrm{W} / \mathrm{m}^{2}$ prior to calling the SERI_QC1 subroutine. See Section 9.1.1 (page 146) for conversions from several common measurement units to $\mathrm{W} / \mathrm{m}^{2}$. Missing data should be indicated by an input value in excess of $8000 \mathrm{~W} / \mathrm{m}^{2}$ (e.g., 9900.00).

The QCFIT program allows you to select from a list of standard measurement units or use the default of $\mathrm{W} / \mathrm{m}^{2}$.

Date and time must accompany each measurement as outlined in sections for each program.

QCFIT generates geographical and irradiance limits for each site, which are stored in the QC-zero configuration files (described in Section 7.3), and SERI_QC1 uses the QC-zero files. 


\subsection{QC-zero Configuration Files}

For each measurement site, QCFIT produces and SERI_QCl uses a special configuration file that contains geographical information about the site and boundary information defining the limits of expected solar radiation values. This file, known as the QC-zero file, is named in the following manner:

$$
\text { S_<site ID>.QCO }
$$

The <site ID> is a unique identifier made up of valid file name characters for the computer operating system in use. For the PC, the <site ID> is limited to 6 characters; for the VAX, it is limited to 20 characters. QCFIT automatically creates the QC-zero file and names it in the above manner based on the site identifier provided by the user.

An annotated sample QC-zero file is shown in Figure 7-1 and explained in Table 7-3.

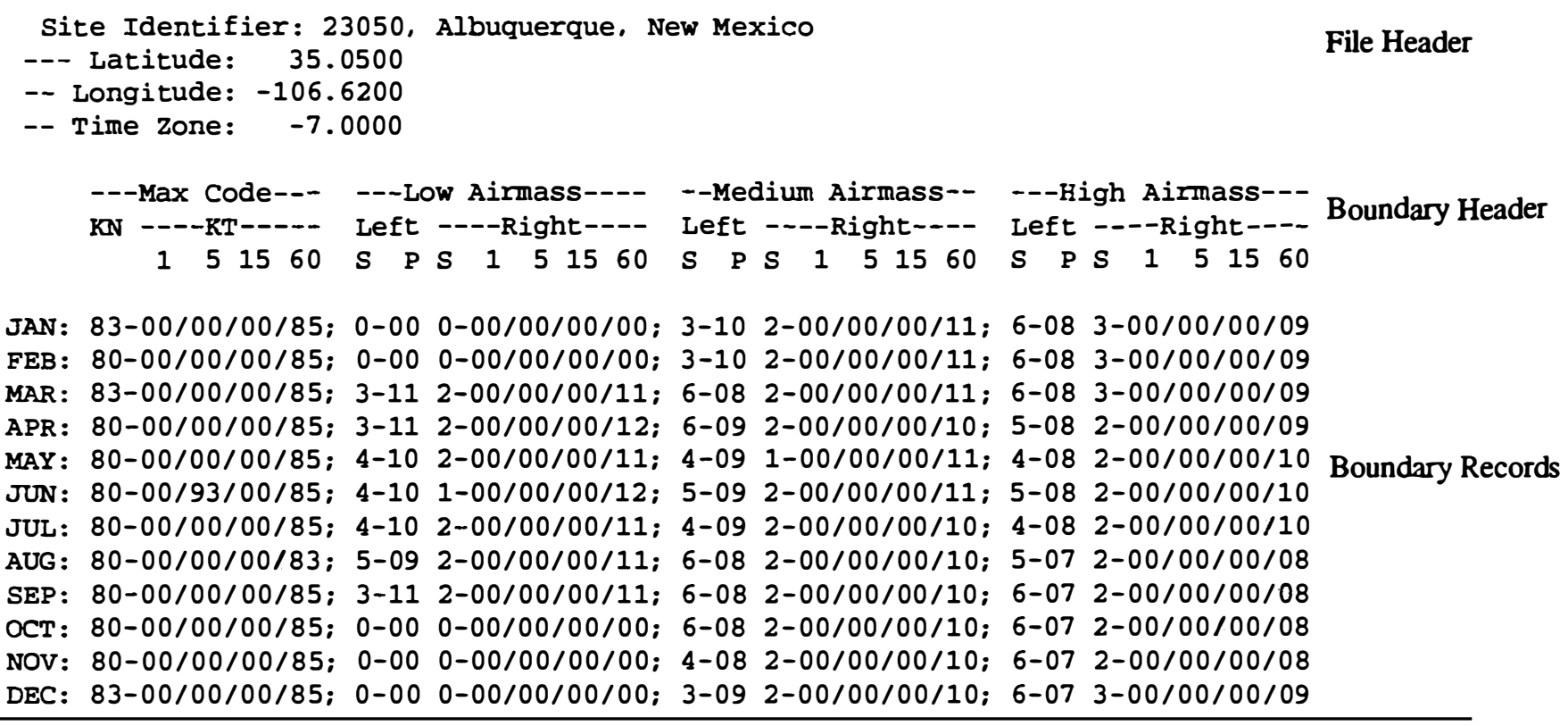

Figure 7-1. Annotated sample QC-zero file 
Table 7-3. Description of QC-zero File (continued)

\begin{tabular}{|c|c|c|c|}
\hline Line & $\begin{array}{l}\text { Character } \\
\text { Position }\end{array}$ & $\begin{array}{l}\text { FORTRAN } \\
\text { Format }\end{array}$ & Field Description \\
\hline$(1-9)$ & & & (FILE HEADER) \\
\hline 1 & $1-80$ & A80 & $\begin{array}{l}\text { Site identifier and brief site description. This entry is } \\
\text { for documentation only; not used by SERI_QCl or } \\
\text { QCFIT (but created by QCFIT). By SERI_QC1 con- } \\
\text { vention, this line contains the site identifier used in the } \\
\text { subroutine, followed by a more general description of } \\
\text { the station. }\end{array}$ \\
\hline 2 & $1-13$ & A13 & Text; identifies the latitude line. \\
\hline & 14 & Al & The ":" character; used as a position reference. \\
\hline & $15-24$ & F10.4 & $\begin{array}{l}\text { Site latitude in decimal degrees (positive north, nega- } \\
\text { tive south). }\end{array}$ \\
\hline 3 & $1-13$ & A13 & Text; identifies the longitude line. \\
\hline & 14 & Al & The ":" character; used as a position reference. \\
\hline & $15-24$ & F10.4 & $\begin{array}{l}\text { Site longitude in decimal degrees (positive east, nega- } \\
\text { tive west). }\end{array}$ \\
\hline 4 & $1-13$ & A 13 & Text; identifies the time zone line. \\
\hline & 14 & A 1 & The ":" character; used as a position reference. \\
\hline & $15-24$ & F10.4 & $\begin{array}{l}\text { The time zone; the number of hours local time leads } \\
\text { (+) or lags (-) Universal Time (UT), e.g., eastem stan- } \\
\text { dard time }=-5 \text {. This is based on the actual local time } \\
\text { used for the data collection even if it is not consistent } \\
\text { with the site longitude. Half-hour or other decimal } \\
\text { time zones may be used, if necessary. }\end{array}$ \\
\hline 5 & (blank) & & \\
\hline $6-8$ & $1-80$ & A80 & Three headers describing the boundary records. \\
\hline 9 & (blank) & & \\
\hline$(10-21)$ & & & (BOUNDARY RECORDS) \\
\hline $10-21$ & $1-3$ & A3 & $\begin{array}{l}\text { Three-character month abbreviation (e.g., "AUG" for- } \\
\text { August). Must be spelled and capitalized as shown in } \\
\text { Figure } 7-1 \text {. }\end{array}$ \\
\hline- & $6-7$ & $\mathbf{1 2}$ & Kn maximum. \\
\hline & $9-10$ & 12 & $\mathrm{Kt}$ maximum for 1-minute integration category. \\
\hline & $12-13$ & 12 & Kt maximum for 5-minute integration category. \\
\hline & $15-16$ & I2 & Kt maximum for 15 -minute integration category. \\
\hline & $18-19$ & 12 & $\mathrm{Kt}$ maximum for 60 -minute integration category. \\
\hline
\end{tabular}


Table 7-3. Description of QC-zero File (concluded)

\begin{tabular}{|c|c|c|c|}
\hline Line & $\begin{array}{l}\text { Character } \\
\text { Position }\end{array}$ & $\begin{array}{l}\text { FORTRAN } \\
\text { Format }\end{array}$ & Field Description \\
\hline & 22 & I1 & Low air mass left boundary shape. \\
\hline & $24-25$ & $\mathbf{1 2}$ & Low air mass left boundary position. \\
\hline & 27 & Il & Low air mass right boundary shape. \\
\hline & $29-30$ & $\mathbf{I} 2$ & $\begin{array}{l}\text { Low air mass right boundary position for } \\
\text { 1-minute integration category. }\end{array}$ \\
\hline & $32-33$ & I2 & $\begin{array}{l}\text { Low air mass right boundary position for } \\
5 \text {-minute integration category. }\end{array}$ \\
\hline & $35-36$ & I2 & $\begin{array}{l}\text { Low air mass right boundary position for } \\
15 \text {-minute integration category. }\end{array}$ \\
\hline & $38-39$ & I2 & $\begin{array}{l}\text { Low air mass right boundary position for } \\
60 \text {-minute integration category. }\end{array}$ \\
\hline & 42 & I1 & Medium air mass left boundary shape. \\
\hline & $44-45$ & I2 & Medium air mass left boundary position. \\
\hline & 47 & I1 & Medium air mass right boundary shape. \\
\hline : & $49-50$ & $\mathbf{1 2}$ & $\begin{array}{l}\text { Medium air mass right boundary position for } \\
\text { 1-minute integration category. }\end{array}$ \\
\hline & $52-53$ & $\mathrm{I} 2$ & $\begin{array}{l}\text { Medium air mass right boundary position for } \\
5 \text {-minute integration category. }\end{array}$ \\
\hline & $55-56$ & I2 & $\begin{array}{l}\text { Medium air mass right boundary position for } \\
15 \text {-minute integration category. }\end{array}$ \\
\hline & $58-59$ & $\mathrm{I} 2$ & $\begin{array}{l}\text { Medium air mass right boundary position for } \\
60 \text {-minute integration category. }\end{array}$ \\
\hline & 62 & I1 & High air mass left boundary shape. \\
\hline & $64-65$ & $\mathrm{I} 2$ & High air mass left boundary position. \\
\hline & 67 & I1 & High air mass right boundary shape. \\
\hline & $69-70$ & I2 & $\begin{array}{l}\text { High air mass right boundary position for } \\
\text { 1-minute integration category. }\end{array}$ \\
\hline & $72-73$ & $\mathbf{I} 2$ & $\begin{array}{l}\text { High air mass right boundary position for } \\
\text { 5-minute integration category. }\end{array}$ \\
\hline & $75-76$ & $\mathrm{I} 2$ & $\begin{array}{l}\text { High air mass right boundary position for } \\
\text { 15-minute integration category. }\end{array}$ \\
\hline & $78-79$ & $\mathrm{I} 2$ & $\begin{array}{l}\text { High air mass right boundary position for } \\
60 \text {-minute integration category. }\end{array}$ \\
\hline 22 & (blank) & & \\
\hline (23-end) & & & (USER NOTES) \\
\hline 23-end & $1-80$ & A80 & $\begin{array}{l}\text { User notes in ASCII text with line length of } 80 \text { charac- } \\
\text { ters or less and lines terminated with a }\langle C R>\text {. }\end{array}$ \\
\hline
\end{tabular}


An ASCII text editor, such as the VAX EDT or MS-DOS EDIT editor, can be used to add notes or comments to the end of the QC-zero file.

Word processing programs or editors that impose a non-ASCII format on a document should never be used to edit the QC-zero files.

The notes should begin at least two lines after the December boundary record and may continue without limit. Lines must be 80 characters or less in length.

SERI_QC1 and QCFIT expect a rigid format in the QC-zero file; thus, only users with a thorough knowledge of the format should attempt to make changes to the boundary data with a text editor. Normally, only QCFIT should be used to make changes. 


\subsection{SOLPOS Subroutine}

The SOLPOS subroutine, which is included in the source code for both SERI_QC1 and QCFIT, takes geographical and time information and computes SOLZEN (solar zenith angle), ETR (extraterrestrial radiation) and ETRN (extraterrestrial radiation normal). Figure 7-2, on the following pages, illustrates this subroutine.

There is a slight difference between the SOLPOS subroutine included in the SERI_QC1 subroutine and that included in the QCFIT program: The SOLPOS subroutine in SERI_QC1 has an internal solar constant of $1367.0 \mathrm{~W} / \mathrm{m}^{2}$, whereas the SOLPOS subroutine in QCFIT receives the solar constant as a passed parameter.

The SOLPOS subroutine uses the NREL Technology and Resource Assessment Branch (Golden, $\mathrm{CO}$ ) consensus solar position and intensity algorithms, as documented in the source code listing. 


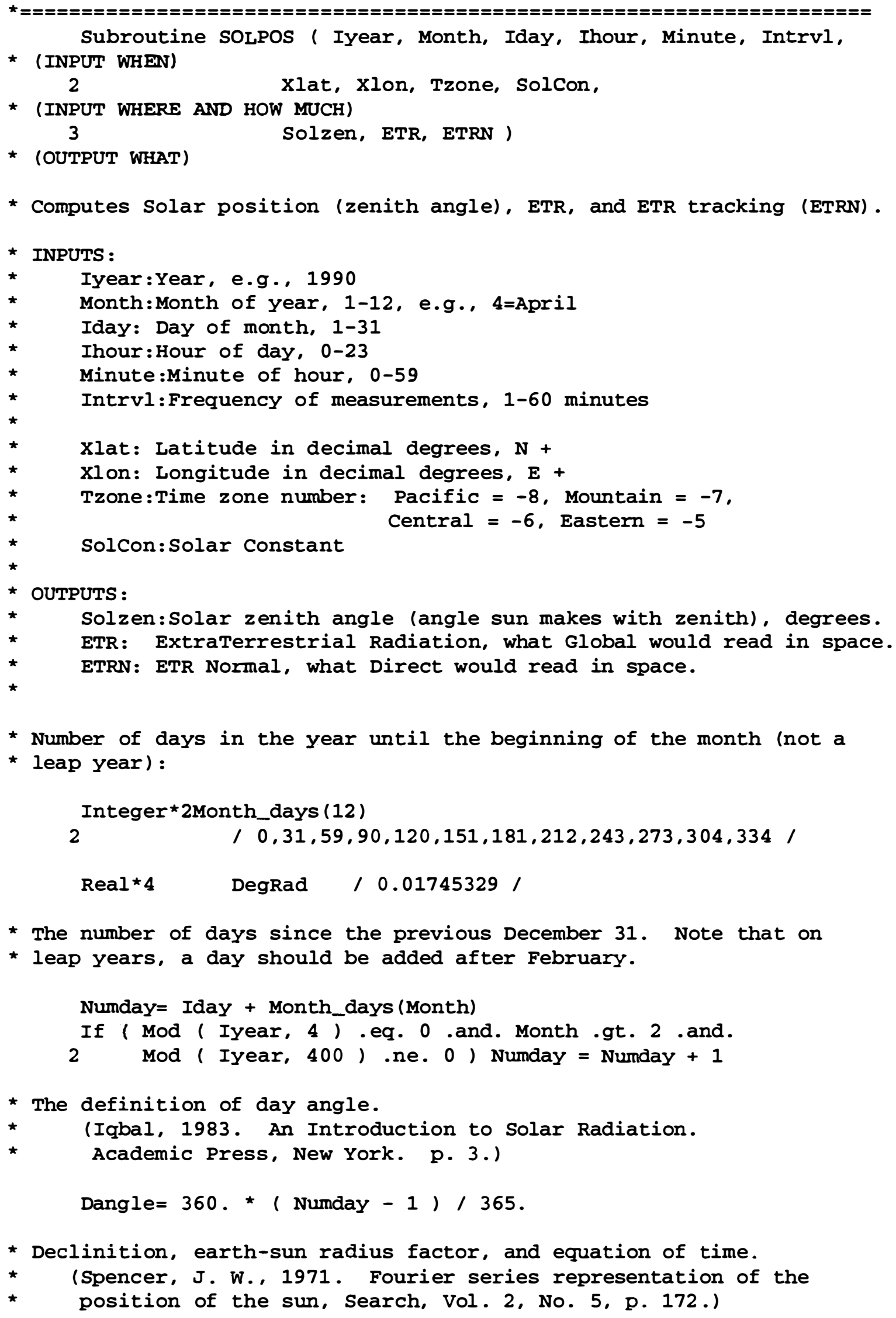

* Number of days in the year until the beginning of the month (not a

* leap year): 


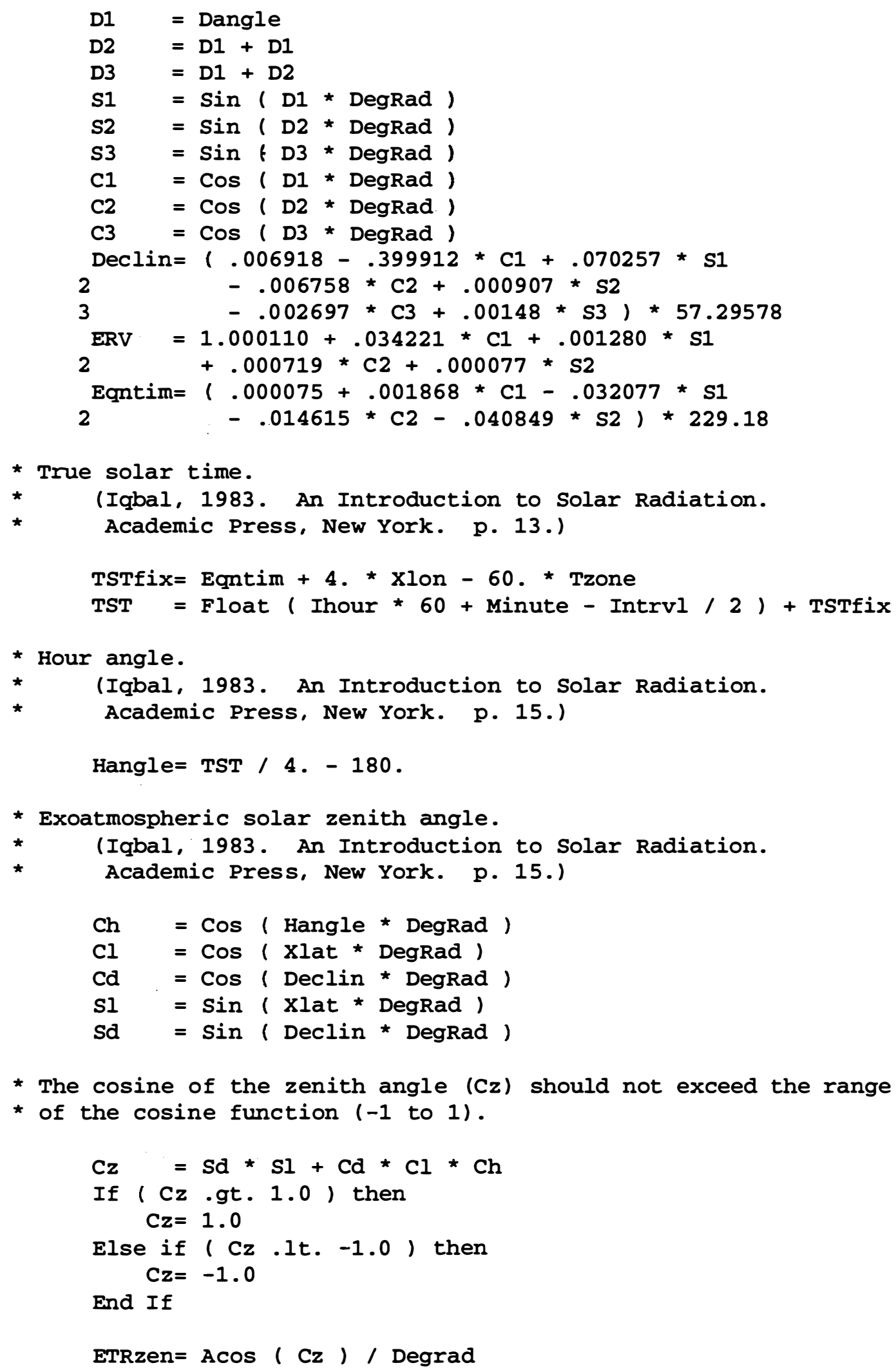

Figure 7-2. SOLPOS subroutine (continued) 
* Exoatmospheric solar elevation angle (needed for refraction correction).

E $\quad=90.0-$ ETRzen

* Refraction correction on elevation angle.

* (zimmerman, John C., Sun-pointing Programs and their Accuracy,

* SAND81-0761, Experimental Systems Operation Division 4721, Sandia

* National Laboratories, Albuquerque, New Mexico, May 1981)

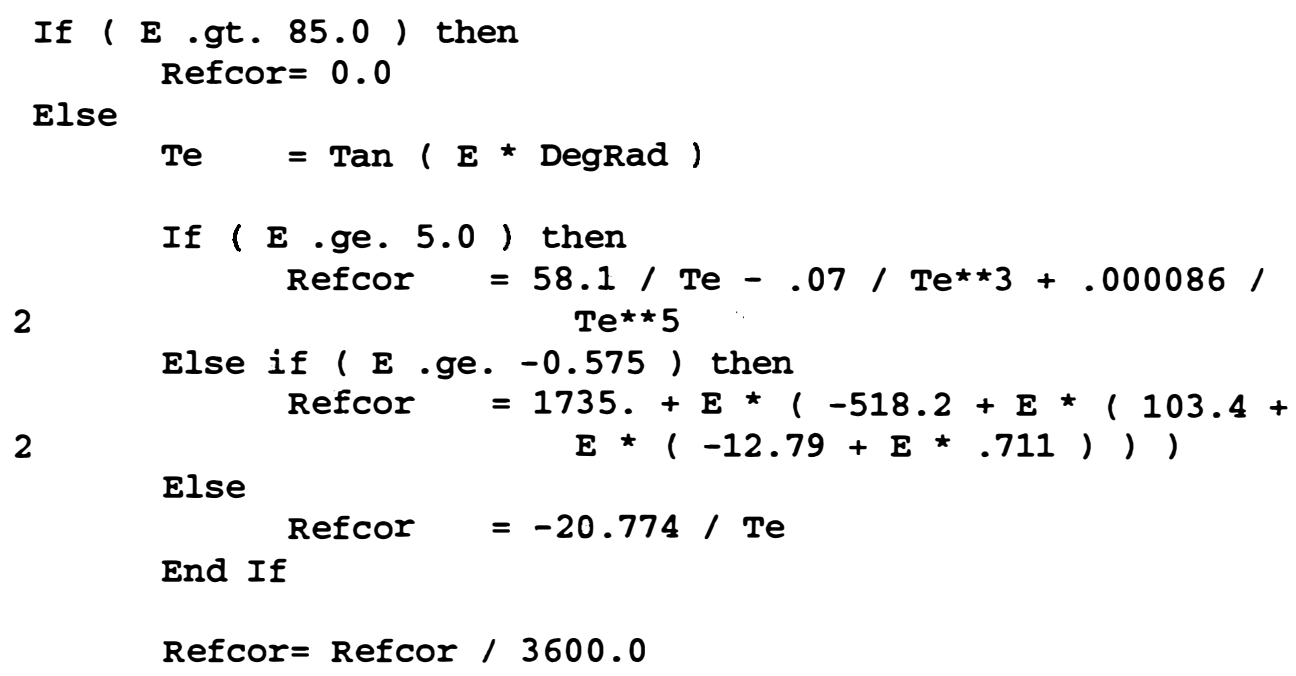

Figure 7-2. SOLPOS subroutine (concluded) 


\subsection{Gompertz Curves}

\subsubsection{Defining Gompertz Curves}

The right and left Gompertz curves are identified numerically by shape and position. For each curve shape, there are 20 horizontal positions spaced at 0.025 $\mathrm{Kt}$ intervals starting at the left with position 1. In Chapter 5, Figure 5-2 (page 36) shows the six Gompertz shapes used for the left boundary, and Figure 5-3 (page 36) shows the five Gompertz shapes used for the right boundary. All of the curves shown are in position 10.

For the set of curves that form the left boundaries, position 1 (the leftmost position) of each shape passes through $\mathrm{Kt}=0.35, \mathrm{Kn}=0.4$. Position 2 passes through the point $0.025 \mathrm{Kt}$ to the right $(0.375,0.4)$; position 3 through $(0.4,0.4)$; and so on through position 20 , which passes through $(0.825,0.4)$. As an example, Figure 5-4 (page 37) shows the 20 positions for left shape 1.

For the set of curves that form the right boundaries, position 1 (the leftmost curve) of all shapes passes through $\mathrm{Kt}=0.475, \mathrm{Kn}=0.4$. Position 2 passes through the point $0.025 \mathrm{Kt}$ to the right $(0.5,0.4)$; position 3 through $(0.525,0.4)$; and so on through position 20 , which passes through $(0.950,0.4)$.

\subsubsection{Storing Gompertz Data}

Block data Gompertz is used by both SERI_QC1 and QCFIT to store Gompertz curve data. The data describe position 1 (the leftmost position) for each shape and provide for 10 right-hand shapes and 10 left-hand shapes.

Shapes 1 through 6 are defined for the left-hand data blocks, and shapes 1 through 5 are defined for the right-hand data blocks. The remaining blocks are reserved for future use by NREL and should not be considered as "unused."

The data are stored as real values for $\mathrm{Kt} * 100$ as a Gompertz function of integer $\mathrm{Kn} * 100$ for each shape, position 1 . 



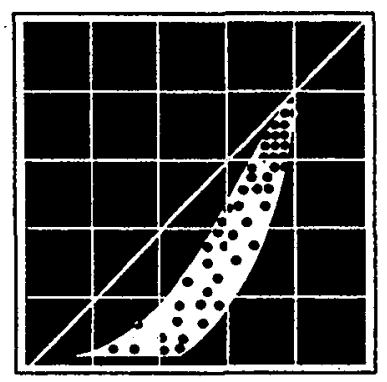

\section{Chapter 8 \\ QCFIT Software Users Guide}

\section{Contents}

8.1 Explanation about the Instructions .................................................. 99

8.2 Required Input Data Files.............................................................. 100

8.2.1 Using the Default QCFIT Data Format ................................... 100

8.2.2 Modifying the QCFIT Defaults ............................................... 102

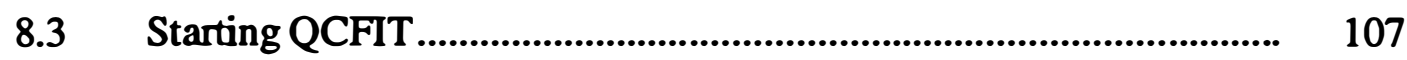

8.4 Editing the Gompertz Boundaries .................................................... 115

8.4.1 Understanding the QCFIT Screen ....................................... 117

8.4.2 Understanding the Scatter Plot Display................................... 118

8.4.3 Choosing Menu Box Editing Options ...................................... 119

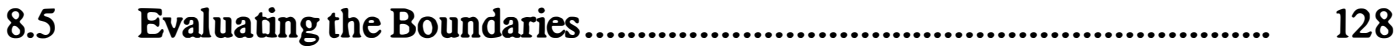

8.5.1 Understanding the Effects of Air Mass .................................. 128

8.5.2 Considering the Effects of Climate and Season ....................... 129

8.5.3 Assessing Data Density .......................................................... 129

8.5.4 Previsualizing the Boundaries ................................................ 129

8.5.5 Examples ................................................................................... 130

8.6 Error Messages .................................................................................. 131

8.7 QCFIT as a Diagnostic Tool......................................................... 132

8.7.1 Understanding Tilt Effects....................................................... 132

8.7.2 Recognizing Time and Position Errors..................................... 134

8.7.3 Detecting Calibration Errors..................................................... 138

8.7.4 Detecting Misadjustment of Shadowbands ............................ 139

8.7.5 Recognizing the Effects of Snow, Frost, and Dirt................... 139 


\section{Overview}

QCFIT is a stand-alone utility to assist users of SERI QC in selecting $\mathrm{Kt}_{\max }$ and $\mathrm{Kn}_{\max }$ limits and Gompertz boundaries that define the limits of expected solar irradiance data for a solar monitoring site. By examining subsets of a site's historical solar data, best-fit limits and Gompertz boundaries can be selected for each of the three SERI QC air mass ranges associated with a station-month. The data output by QCFIT is required by the SERI_QC1 subroutine to assess global, direct, and diffuse solar irradiance data.

QCFIT displays a Kt versus Kn scatter plot of the data on the computer screen and provides several options for fitting predefined curve shapes to the plot. If no limits have been defined, QCFIT automatically makes an initial fit. The user may adjust the automatic fit or use other boundaries based on experience and known attributes of the data. 


\subsection{Explanation about the Instructions}

These instructions contain information about both the VAX and PC versions of the software. When instructions for one version are unique, the text is marked with a $P C$ or VAX in the margin and the duration of the text is marked with a vertical bar, as shown below:

VAX The VAX version allows a site ID of 20 characters or less.

PC The PC version allows a site ID of 6 characters or less.

Notes in parentheses to the right of commands and other user input are for information about the procedure. Do not enter these parenthetical remarks.

Variables, such as file names, appear in angle brackets. For example, the statement "I don't have <site ID>" means this statement appears on the screen with the actual site identifier name within the angle brackets. The abbreviations for certain keyboard keys are also enclosed in angle brackets. For example, a Carriage Retum (or Enter) appears in the documentation as $\langle\mathrm{CR}\rangle$.

Screen displays and prompts are boxed as shown below:

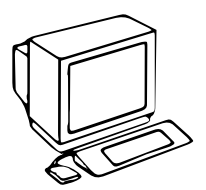

$$
\operatorname{LEFT}(Y, N) \quad[Y]
$$

All keyboard input requires an entry followed by a $<\mathrm{CR}>$.

For brevity, all Yes and No prompts on the screen are abbreviated as Y or N. For clarity, this document refers to the responses as Yes and No, although the program allows you to enter either a " $Y$ " followed by a $<C R>$ or an " $N$ " followed by a $<C R>$. Default responses are enclosed in square brackets and may be selected by entering $<\mathrm{CR}>$ alone. In the previous example, the default answer is Yes.

All inputs for QCFIT are case insensitive: The program interprets uppercase or lowercase alpha characters the same. An exception is text entered for the site identifier and site description. These entries are stored in the QC-zero file as entered. Both these entries are for documentation purposes and are not used by the program. 


\subsection{Required Input Data Files}

This section discusses the data and formats required for running QCFIT, as well as the method to modify QCFIT to accept irradiance data in other formats.

\subsubsection{Using the Default QCFIT Data Format}

QCFIT requires geographical data for the station under examination. Initial runs for the station require user input of this data; subsequent runs use the stored data from the QC-zero file.

The following data are required:

- A unique site identifier (ID) and short site description

VAX The VAX version allows a site ID of 20 characters or fewer.

$P C \quad$ The PC version allows a site ID of 6 characters or fewer.

- $\quad$ Site latitude (in decimal degrees)

- $\quad$ Site longitude (in decimal degrees)

- $\quad$ Time zone (offset from Greenwich, positive east, negative west). This must be based on the actual local time used in recording the data, even if this is inconsistent with the site longitude. Fractional time zones may be used.

Example using Albuquerque, NM: Site ID “ABQ” latitude 35.05 north, longitude 106.62 west, time zone - 7.0 (mountain standard time).

For each measurement, QCFIT requires the solar irradiance data and time/date information described in Table 8-1. The default FORTRAN format is F5.0, 4F3.0, $3 F 10.2$, and the default unit of measurement is $\mathrm{W} / \mathrm{m}^{2}$. See Section 8.2 .2 for the procedure to change these defaults. 
Table 8-1. Default QCFIT Input Data Description

\begin{tabular}{|c|c|l|}
\hline $\begin{array}{c}\text { Character } \\
\text { Position }\end{array}$ & $\begin{array}{c}\text { FORTRAN } \\
\text { Format }\end{array}$ & \multicolumn{1}{|c|}{ Field Description } \\
\hline \hline $1-5$ & F5.0* & $\begin{array}{l}\text { The year that the data were collected (the century portion } \\
\text { of the year need not be present; i.e., "78" suffices for } \\
\text { "1978." }\end{array}$ \\
\hline $6-8$ & F3.0* & The month. Must be a value between 1 and 12. \\
\hline $9-11$ & F3.0* & The day. Must be a valid day of the month. \\
\hline $12-14$ & F3.0* & The hour. A value between 0 and 24, local standard time. \\
\hline $15-17$ & F3.0 & The minute. A value between 0 and 59. \\
\hline $18-27$ & F10.2 & $\begin{array}{l}\text { Global horizontal irradiance in } \mathrm{W} / \mathrm{m}^{2} \text {. Missing data are } \\
\text { indicated by a value }>8000 .\end{array}$ \\
\hline $28-37$ & F10.2 & $\begin{array}{l}\text { Direct normal irradiance in } \mathrm{W} / \mathrm{m}^{2} . \text { Missing data are indi- } \\
\text { cated by a value }>8000 .\end{array}$ \\
\hline $38-47$ & F10.2 & $\begin{array}{l}\text { Diffuse horizontal irradiance in } \mathrm{W} / \mathrm{m}^{2} \text {. Missing data are } \\
\text { indicated by a value }>8000 .\end{array}$ \\
\hline
\end{tabular}

* Although the date and time values are specified as FORTRAN real numbers, QCFIT uses only the integer portion internally, and fractional values are ignored.

The day runs from 00:01 to 24:00. These fields represent the ending minute of the period during which the measurements were taken. QCFIT requires that these data be stored as a series of sequential line-delineated records in a file.

QCFIT requires measurements from two of the three fundamental solar components: global horizontal, direct normal, and diffuse horizontal. If the data for any component is missing, the field should be filled with a value greater than 8000 $\mathrm{W} / \mathrm{m}^{2}$. (Other values for missing data may be specified, see Section 8.2.2).

The following sample record shows the time, date, and irradiance fields for a measurement that ended at 11:00 May 12, 1979:

$$
\begin{array}{lllllllll}
79 & 5 & 12 & 11 & 0 & 928.03 & 1102.47 & 9900.00
\end{array}
$$

In this example, the global horizontal measurement is $928.03 \mathrm{~W} / \mathrm{m}^{2}$, the direct normal measurement is $1102.47 \mathrm{~W} / \mathrm{m}^{2}$, and the diffuse horizontal measurement is missing.

Data for individual sites should be in individual files for this format. You may place all data for one site in a single file; however, QCFIT runs faster with the data 
broken down into smaller subsets, such as monthly files. The smallest allowable subset is monthly data. If data from more than one year is to be used to determine boundaries for expected measurement values (as is ideally the case), data from a particular month for all years must be in one file (though not necessarily to the exclusion of data from other months). For example, to determine a set of curves for January using data from 1978, 1979, and 1980, the data for January 1978, January 1979 , and January 1980 must be in the same file. The records need not be in chronological or any other order.

\subsubsection{Modifying the QCFIT Defaults}

Reformatting your data to fit default input requirements is inefficient both in terms of time and disk storage. QCFIT uses the default input format described previously for the solar radiation data unless directed otherwise by information in a special file for specifying defaults, QCDEFLT.DAT. Thus, you can tailor the input format to your needs. The procedures outlined in this section require line-oriented records for your data file. Records that span multiple lines cannot be accommodated by QCFIT.

Using an ASCII text editor, create (or edit, if it already exists) a file called QCDEFLT.DAT. This file must reside in the same directory where you will be running the QCFIT program. Insert any of the following left-justified lines in the file:

$P C$

$P C$

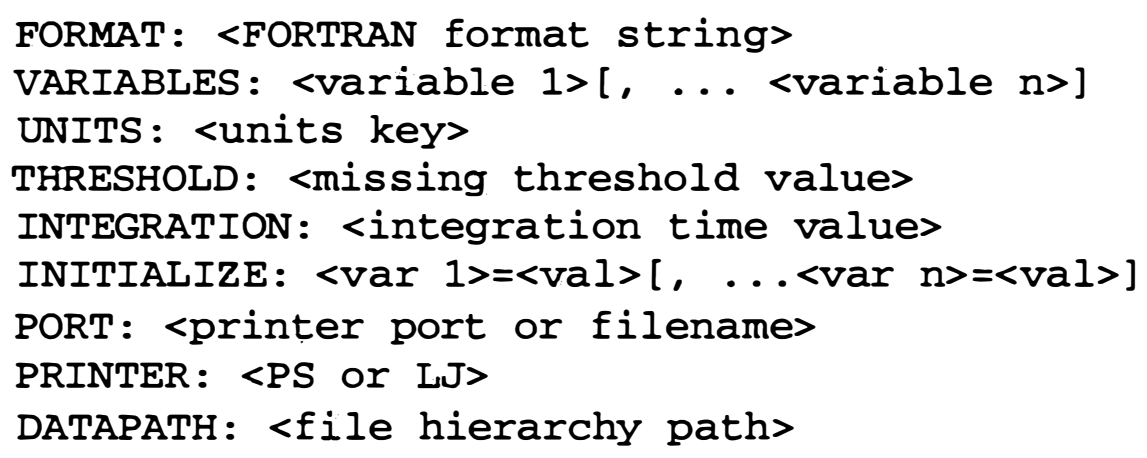

The characters before the colon on each line must be capitalized and spelled exactly as shown. Although the above sample shows the lines indented from the left margin, the entries in your file must start with the first character of the line.

The values following the colon on each line are described in the corresponding section below:

FORMAT: The <FORTRAN format string $>$ must be a valid FORTRAN format statement, including leading and trailing parentheses. All variable formats must be of type real, e.g., F10.2. 
VARIABLES: The variable list contains the eight time and date variables in the order they appear in the input record from your data file. The eight variables are shown in Table 8-2. The variable names must be spelled and capitalized as shown.

Table 8-2. Variable Names

\begin{tabular}{|l|c|}
\hline \multicolumn{1}{|c|}{ Variable } & DEFAULT \\
\hline \hline YEAR & 1901 \\
\hline MONTH & none \\
\hline DAY & none \\
\hline HOUR & none \\
\hline MINUTE & 0 \\
\hline GLOBAL & 9900.0 \\
\hline DIRECT & 9900.0 \\
\hline DIFFUSE & 9900.0 \\
\hline
\end{tabular}

If a variable does not appear in your data record, it may be left out of the variable list, and it will take on the default value shown in the table. All variables must be assigned a value, whether from your data file, from the default in Table 8-2, or through the initialization line described below.

UNITS: The <units key> value is a two or three character key from Table 8-3 that specifies the measurement unit of the solar radiation data. The key must be spelled and capitalized as shown.

Table 8-3. Solar Radiation Units

\begin{tabular}{|l|l|}
\hline \multicolumn{1}{|c|}{ Key } & \multicolumn{1}{c|}{ Units Description } \\
\hline \hline WM & Watts per square meter $\left(\mathrm{W} / \mathrm{m}^{2}\right)$ [default] \\
\hline KWH & Kilowatt-hours per square meter $\left(\mathrm{kWh} / \mathrm{m}^{2}\right)$ \\
\hline $\mathrm{JM}$ & Joules per square meter $\left(\mathrm{J} / \mathrm{m}^{2}\right)$ \\
\hline KJM & Kilojoules per square meter $\left(\mathrm{kJ} / \mathrm{m}^{2}\right)$ \\
\hline MJM & Megajoules per square meter $\left(\mathrm{MJ} / \mathrm{m}^{2}\right)$ \\
\hline
\end{tabular}

If no units are specified, QCFIT uses the default of $\mathrm{W} / \mathrm{m}^{2}$ (WM). 
THRESHOLD: The <missing threshold value> causes QCFIT to treat solar radiation data as missing. If the value is positive, any data greater than or equal to the threshold is treated as missing; if the value is negative, any data less than or equal to the threshold is treated as missing. If no threshold value is specified, QCFIT uses 8000.0 as the threshold value.

INTEGRATION: The <integration time value $>$ indicates the time interval in minutes over which each solar radiation measurement was integrated. If no value is specified, QCFIT uses the default of 60 minutes (which may be overridden by the user during program use). The <integration time value > must be an integer.

INITIALIZE: The variable list ( $\operatorname{var} n$ ) contains variable names from Table 8-2 that you may wish to initialize. This capability allows you to set values for the optional variables that may not be included in your data files. These names should not appear in the list for the "VARIABLE:" defaults. YEAR, MONTH, DAY, and MINUTE must be entered as integer values.

The format for this line is the variable name, an equals sign ("="), and an initializing value, followed by a comma if other variables are listed. There should be no space between the variable name, the equals sign, and the value.

PC PORT: The <printer port or filename > specification names the PC printer port or file name where you want to send a hard copy image of the QCFIT scatter plot. It must be a valid MS-DOS device or file name, such as "COM1" or "IMAGE.PS." The default is "LPT1." This specification applies only to the (P)rint Screen command detailed in Section 8.4.3, and the output is either PostScript commands for a Postscript printer or PCLHP.GL commands for a Hewlett-Packard LaserJet III or LaserJet 4. See the PRINTER description below for how to specify printer output. Note: In some contexts, ports are referenced with a port name followed by a colon, such as "COM1::" Do not specify a port name with a trailing colon in this application. If you do, the program may hang and your work will be lost.

PC PRINTER: The $<$ PS or $\mathrm{LJ}>$ specification code selects the type of printer language for hard copy output. PS specifies PostScript printer output (the default), and LJ specifies PCL/HPGL output for a Hewlett-Packard LaserJet III or LaserJet 4. Note: Also see the (P)rint Screen command in Section 8.4.2 (page 118) for a method to print to other graphics printers.

DATAPATH: The <file hierarchy path> is the directory path to your data files. The default path is the current logged directory. Although a path name may also be entered as part of a file name by the user, doing so will not override this DATAPATH default specification. 


\section{Example 1}

To duplicate the default format described in the previous section, you would enter the following lines in the QCDEFLT.DAT file:

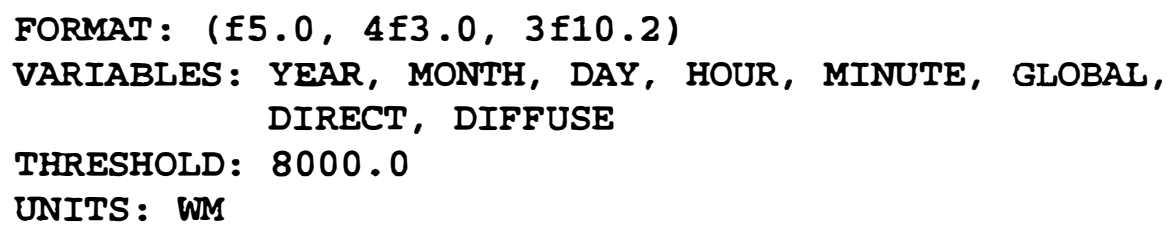

Note that the "VARIABLES" line in this example is split to the next line because of space limitations. Such a split should not occur in the QCDEFLT.DAT file.

\section{Example 2}

Your data, in $\mathrm{J} / \mathrm{m}^{2}$, does not contain a year time stamp (collected in 1980) or data for diffuse measurements, and contains other data before the Direct and Global fields. Missing data are denoted with a value of -9900.0 . The data record looks like this:

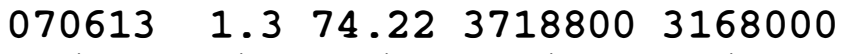

In the QCDEFLT.DAT file, you enter:

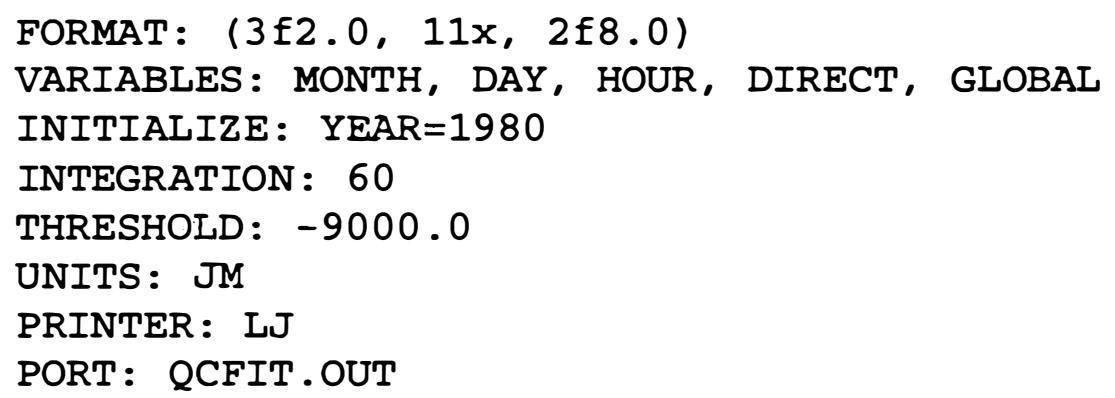

The last two lines specify that the printer output will be for a Hewlett-Packard LaserJet printer rather than the default PostScrir: printer, and that the printer output will be stored in a file called QCFIT.OUT rather than being sent to the default LPT1 printer port. 
When accommodating a format, remember that your data must have the date and time-information associated with each measurement record. Further, QCFIT will operate correctly only when the time and date values must reflect the ending time of the data collection, as opposed to a beginning time or midpoint. 


\subsection{Starting QCFIT}

VAX These instructions assume the QCFIT source code has been compiled and linked in an executable form. Consult your FORTRAN manual for more information about compiling and linking FORTRAN programs.

Figure 8-1 shows a simplified flow diagram for using QCFIT to create a QC-zero file that contains the necessary geographical and Gompertz boundary information for a site.

The following steps guide you through the procedures to specify all parameters and input data for QCFIT.

\section{Step 1. Starting the Program}

Move to the directory where the QCFIT executable file and QC-zero files (if any have been created yet) reside. The solar data files in the QCFIT format need not be in the same directory but must be accessible through file hierarchical specifications. At the system prompt, enter:

$P C \quad$ QCFIT $<\mathrm{CR}>$

VAX RUN QCFIT <CR>

The QCFIT opening screen and component selection prompt appears:

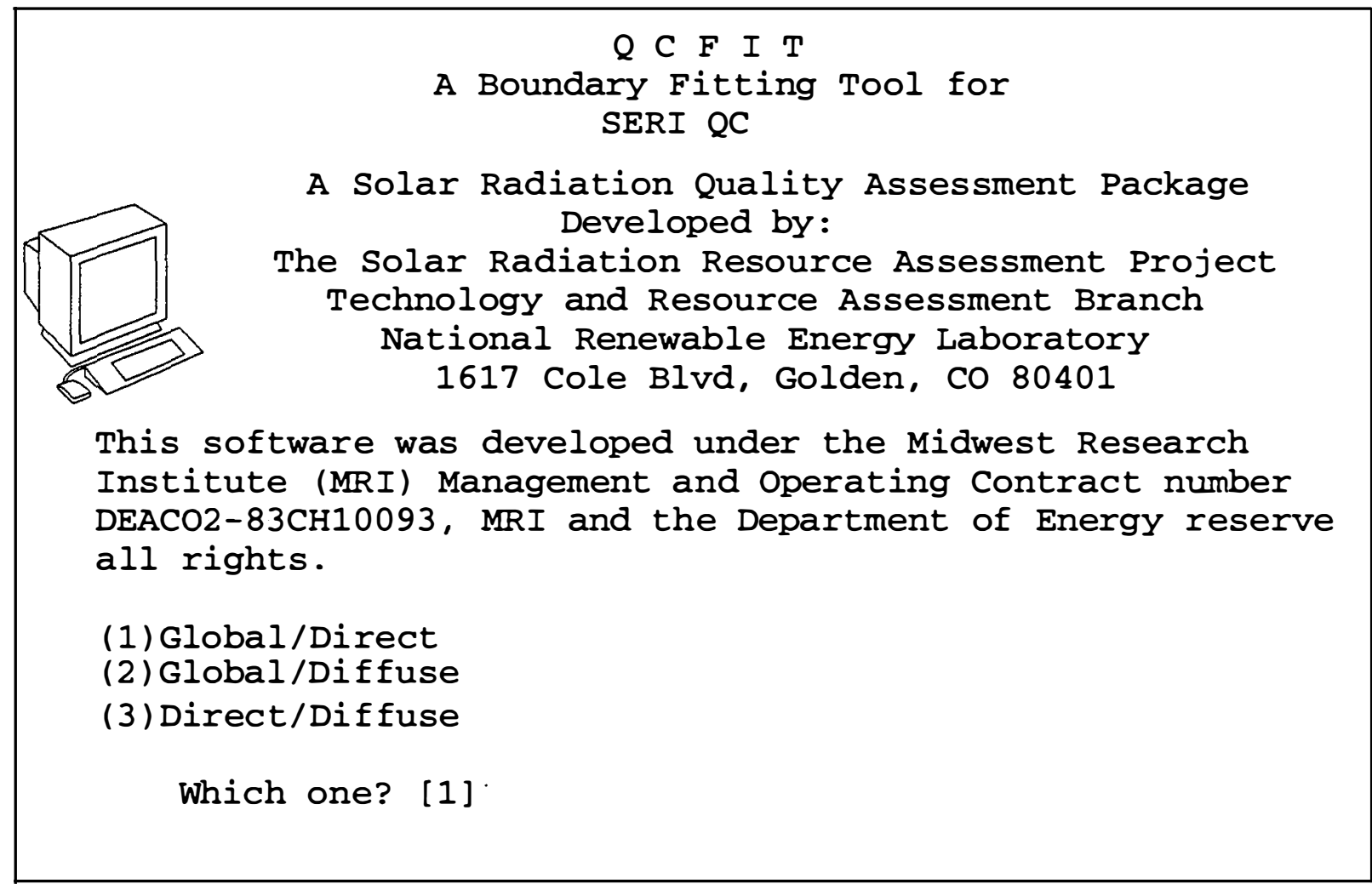




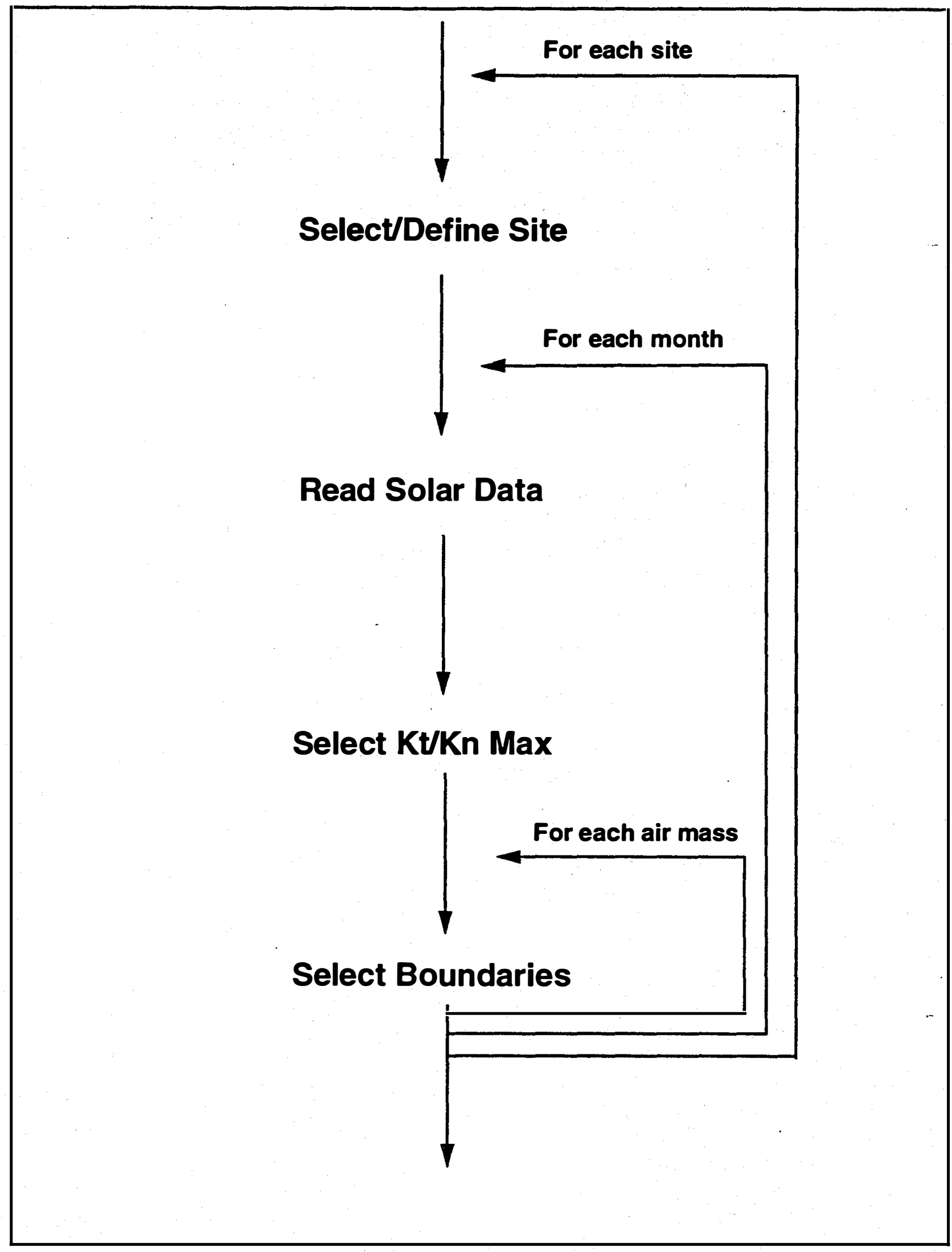

Figure 8-1. Flow diagram for using QCFIT 


\section{Step 2. Choosing Solar Components}

Answer the question on the opening screen with a 1,2 , or 3 , depending on the components present in your data file. (You must have at least two of the three fundamental solar components. ${ }^{1}$ ) If all three components are present in your data set, select (1) Global/Direct. This is the default selection that can be selected by pressing $<\mathrm{CR}>$.

\section{Step 3. Specifying Integration Time}

The prompt for the integration time of the measurements appears as follows:

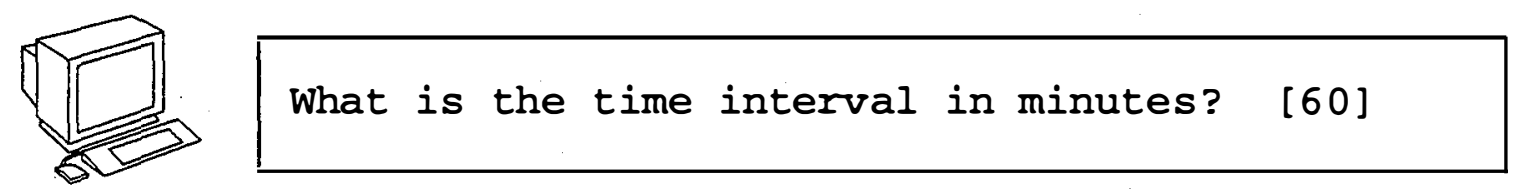

Enter the integration time of your data. The default time appears in the brackets and may be selected by pressing $\langle\mathrm{CR}\rangle$.

QCFIT accepts any integration time between 1 and 60 minutes and saves the information in the QC-zero file in four categories based on integration times. From studies of 1-, 5-, 15- and 60-minute data, we found that the changes in boundary positions and $\mathrm{Kt}_{\max }$ values followed a logarithmic pattern. We also determined that no more than three boundary shifts occurred for this range of integration times. Therefore, the QC-zero file provides for four right-hand boundary positions and four $\mathrm{Kt}_{\max }$ values to accommodate all positions that are likely to be required for data collected using integration times between 1 and 60 minutes. Table 8-4 shows the log-based integration times for each of the four positions in the QC-zero file and the manner in which these are designated in the QC-zero file and QCFIT displays.

Table 8-4. Log-Based Integration Times and Designation

\begin{tabular}{|c|c|c|}
\hline QC-Zero Position & Time Entered & Designated As \\
\hline \hline 1 & $1-2$ minute & 1 minute \\
\hline 2 & $3-8$ minute & 5 minute \\
\hline 3 & $9-32$ minute & 15 minute \\
\hline 4 & $33-60$ minute & 60 minute (hourly) \\
\hline
\end{tabular}

1. QCFIT has limited usef ulness when examining one-component data because two components are required to produce a scatter plot. The only useful feature of QCFIT when using one-component data is to set maximum $\mathrm{K}$ values (Xmax for global or $\mathrm{Y} \max$ for direct). Even this function is of questionable value because QCFIT gives no indication of where to set the values. The best approach when developing a site using onecomponent data is to select an existing site with a similar climate and copy its QC-zero file using the geographical information for the new site. If no template site is available, our experience shows that an Xmax of 88 and a Ymax of 83 are rarely exceeded. 


\section{Step 4. Selecting a Site}

QCFIT requests the site identifier of the data you are examining.

$P C \quad$ The PC site selection prompt appears as follows:

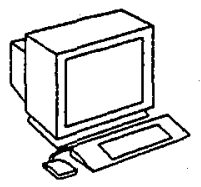

Enter your site identifier:

VAX The VAX site selection prompt appears as follows:

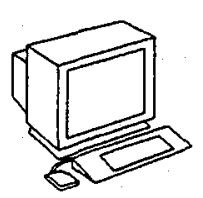

Current active site identifiers:

<site ID> <site ID> <site ID>

<site ID> <site ID> <site ID>

Please enter your site identifier:

Enter the identifier of the site you wish to examine. If you wish to initialize a new site, enter a new unique identifier.

PC For the PC, the identifier must be 6 characters or less.

$V A X$ For the VAX, the identifier must be 20 characters or less.

These characters must be valid file name characters. Consult your operating system documentation for more information about valid characters.

If the identifier you enter does not exist, the following prompt appears:

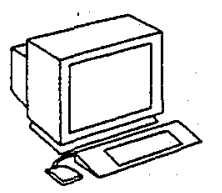

$$
\text { I don't have <site ID>. Is this correct }(Y, N) \text { ? }
$$

If you have entered a new identifier, answer Yes and go to step 5. If you mistyped the entry, enter No.

If you answered No, the original site identifier prompt reappears and you are again asked to enter a site code. Once you successfully enter an existing site identifier, go to step 6. 
If you entered a new identifier, QCFIT prompts you for the required site information to initialize a QC-zero configuration file for the new site:

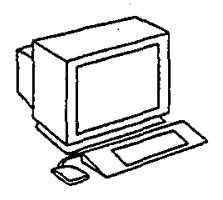

Please give a site description that is not longer than the dashed line:

Enter a short description of the site, such as city and state, that is no longer than the dashed line below the prompt. If the description exceeds the length of the dashed line, it will be truncated. If you do not wish to enter a description, enter $\langle C R>$ only. This description is case sensitive and will be stored in the QC-zero file exactly as entered.

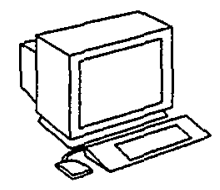

What is the Latitude in degrees (e.g., 40.2693)?

Enter the decimal value for degrees to four decimal places if possible.

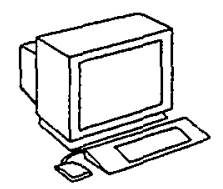

Is this NORTH latitude $(Y, N)$ ?

[Y]

Enter Yes or No, or $<\mathrm{CR}>$ for the default answer in brackets.

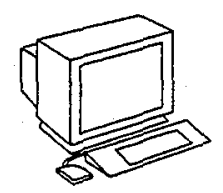

What is the Longitude in degrees (e.g.,95.2711)?

Enter the decimal value for degrees to four decimal places if possible.

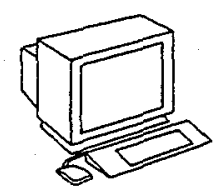

Is this WEST longitude $(Y, N)$ ?

[Y]

Enter Yes or No, or $<C R>$ for the default answer in brackets.

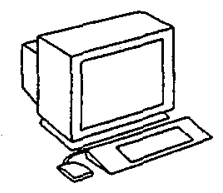

What time zone $(P-8, M-7, C-6, E-5) ?[-6]$ 
Enter the time zone corresponding to the local time used by the station. The default value in brackets reflects the expected time zone based on the site's longitude, which may differ from your site's actual time zone. Enter $\langle C R>$ for the default time zone, or enter an offset from Universal time (Greenwich) using negative values for zones west of Greenwich and positive values for zones east of Greenwich. For example, Pacific time is -8 , mountain time is -7 , central time is -6 , and eastern time is -5 . Note: Some regions of the world have fractional time zones; for example, Newfoundland's time zone is -3.5 . QCFIT accepts fractional time zones.

\section{Step 6. Specifying the Month}

You must specify the month during which the data to be examined were collected. QCFIT prompts:

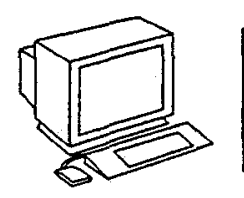

$$
\text { Which month shall be handled }(1-12) ?
$$

Enter a number between 1 and 12 corresponding to the month of the year the data were collected ( 1 corresponds to January, 2 corresponds to February, and so on).

\section{Step 7. Specifying the Data File}

After QCFIT finds or creates the QC-zero site information file, you are prompted for the data file name:

PC On the PC, the prompt for a file name appears as follows:

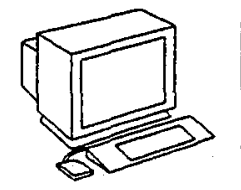

What is the input filename (include extension)?

VAX On the VAX, the prompt for a file name appears as follows:

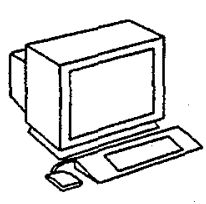

What is the input filename (include extension)? (If you forgot your file, use wildcards $(*, q)$ as in the VAX "DIR" command and get a directory.)

Enter the name of the file holding the solar data (in the QCFIT format) that you wish to use for developing or modifying curves. If the file is not in the current directory, you must include the path to the file as part of the file name or specify a default path to your data as outlined in Section 8.2.2. 
$V A X$ If no file extension is specified, the program assumes a ".DAT" extension. If you enter an ambiguous file specification using wildcard characters *,\%), QCFIT displays a directory listing of files in the current directory matching the file specification. For example, if you enter "FEB*.DAT," the resulting display would show you all the files that match that specification. This allows you to confirm a file name before entering it.

If the file you name cannot be found, QCFIT displays this message:

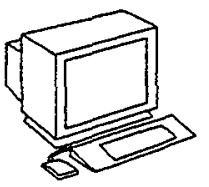

$\star \star \star$ File not found: <filename>

QCFIT prompts again for a file name as shown in the beginning of this step.

If the data file is found, QCFIT reads the file, reporting at 100-line intervals as the data are read from the disk:

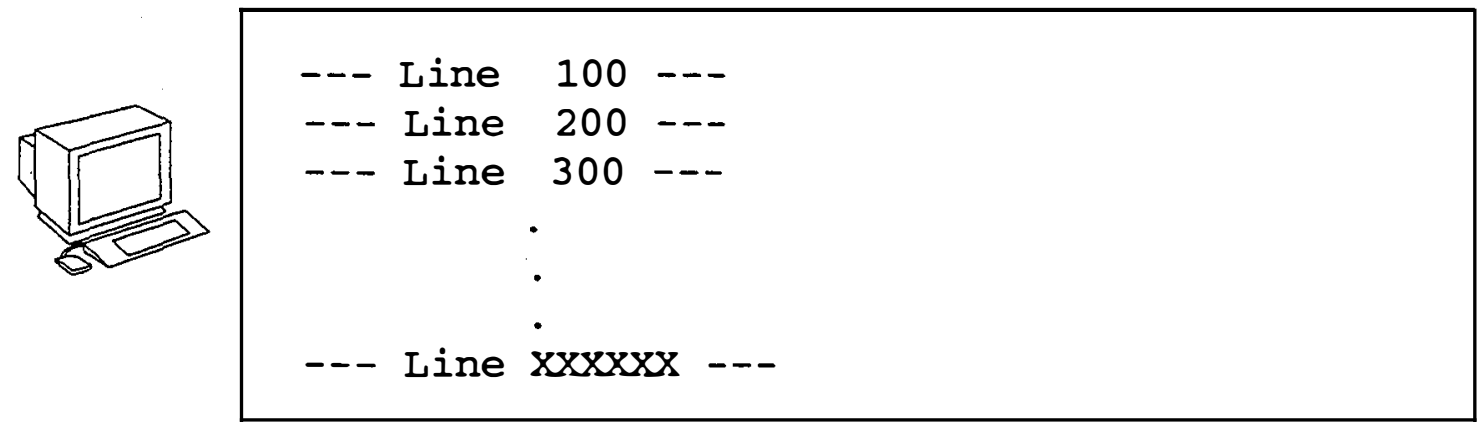

\section{Step 8. Specifying the Air Mass}

As QCFIT reads the solar irradiance data file, data are segregated into three air mass regimes described as Low, Medium and High. The Low air mass range is $\leq 1.25$; the Medium air mass range is $>1.25$ and $\leq 2.5$; and the High air mass is $>2.5$ and $\leq 5.58$ (see Chapter 4, Section 4.2.2, page 25, for further discussion). SERI_QC1 makes the same air mass distinction when evaluating solar data; thus Gompertz boundaries must be selected for each air mass range. Note that no location in the United States has data falling in the Low air mass range for all months.

You are prompted for an initial air mass range:

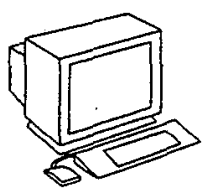

$$
\begin{aligned}
& \text { Which airmass regime, (L) ow, (M) edium, (H)igh? } \\
& {[\mathrm{L}]}
\end{aligned}
$$

Select a category by entering either $\mathrm{L}, \mathrm{M}$, or $\mathrm{H}$. Initially, enter $\mathrm{L}<\mathrm{CR}>$ or $\langle\mathrm{CR}>$ for Low air mass. In later steps, Gompertz boundaries must be defined for all of 
the three air mass ranges for which data exist for the station-month under examination.

After selecting the air mass regime, QCFIT displays a scatter plot of the data and enters the edit mode described in the following section.

Note: At this point, rather than a scatter plot, the following message may appear on the screen:
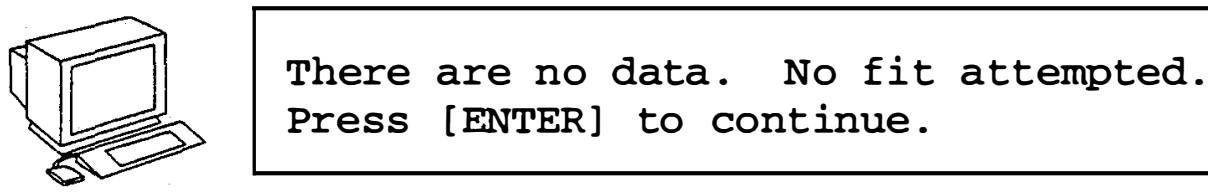

Properly, one should initially fit boundaries to the data in the lowest air mass regime available. However, in winter months, many sites do not have any data in the Low air mass category, resulting in the previous message.

If this is the case, the following commands, which will be explained in greater detail later, should produce a QCFIT screen with a scatter plot and allow you to continue with the next section.
Enter
Explanation
$<\mathrm{CR}>$
(This clears the message.)
A $<\mathrm{CR}>\quad$ (This selects an air mass change.)
$\mathrm{N}<\mathrm{CR}>\quad$ (This bypasses an update routine.)
$\mathrm{M}<\mathrm{CR}>\quad$ (This selects Medium air mass.) 


\subsection{Editing the Gompertz Boundaries}

This section describes the curve fitting and editing capabilities of QCFIT.

For each air mass regime and for each station-month, SERI_QC1 requires a lefthand Gompertz boundary, a right-hand Gompertz boundary, a maximum Kt limit, a maximum $\mathrm{Kn}$ limit, and a maximum $\mathrm{Kd}$ limit. The maximum $\mathrm{Kd}$ limit is calculated from the right-hand Gompertz boundary (see Chapter 6, Section 6.1, page 41) and is not discussed in this section.

If data are found for the month and air mass regime selected earlier, QCFIT displays a $\mathrm{Kt}$ versus $\mathrm{Kn}$ scatter plot of the data. If no boundaries previously have been selected for the site, QCFIT attempts an initial fit of the Gompertz curves.

If boundaries for the same station-month have already been selected based on other integration times, the automatic curve-fitting algorithm is limited to selecting the position of the right-hand boundary. This is necessary because the right-hand shape, and the left-hand shape and position, are shared by all integration times and already have been determined during examination of the data from other integration times.

This limitation on automatic curve fitting and selection helps prevent inadvertent boundary changes for data that are not part of the data set under examination. However, you may override this restriction and make any desired changes by using the Select option described in Section 8.4.3. This might be necessary if boundaries previously selected for other integration times are suspect.

PC Figure 8-2 illustrates the QCFIT editing screen for the PC.

VAX Figure 8-3 illustrates the QCFIT editing screen for the VAX. 


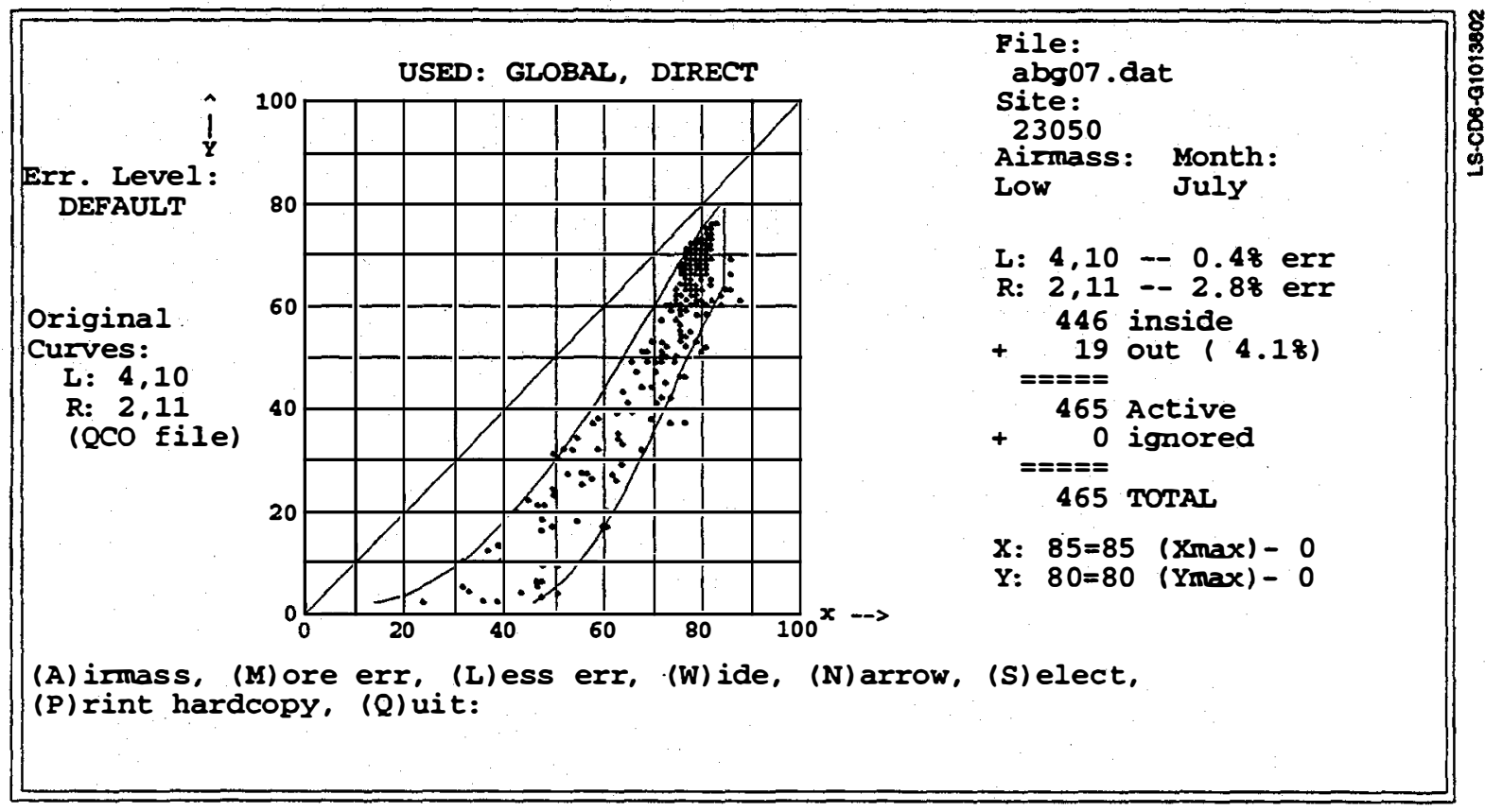

Figure 8-2. PC QCFIT editing screen

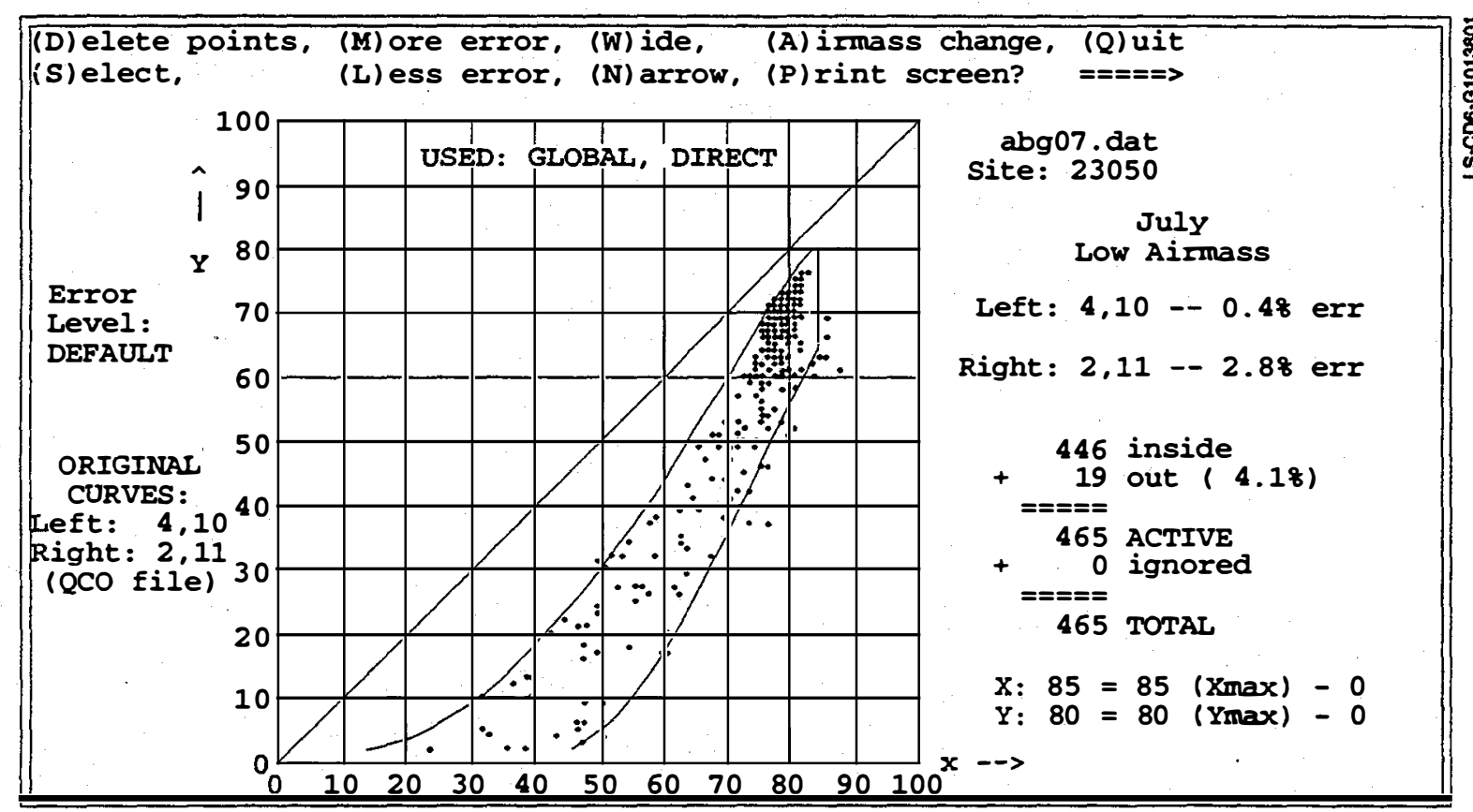

Figure 8-3. VAX QCFIT editing screen 


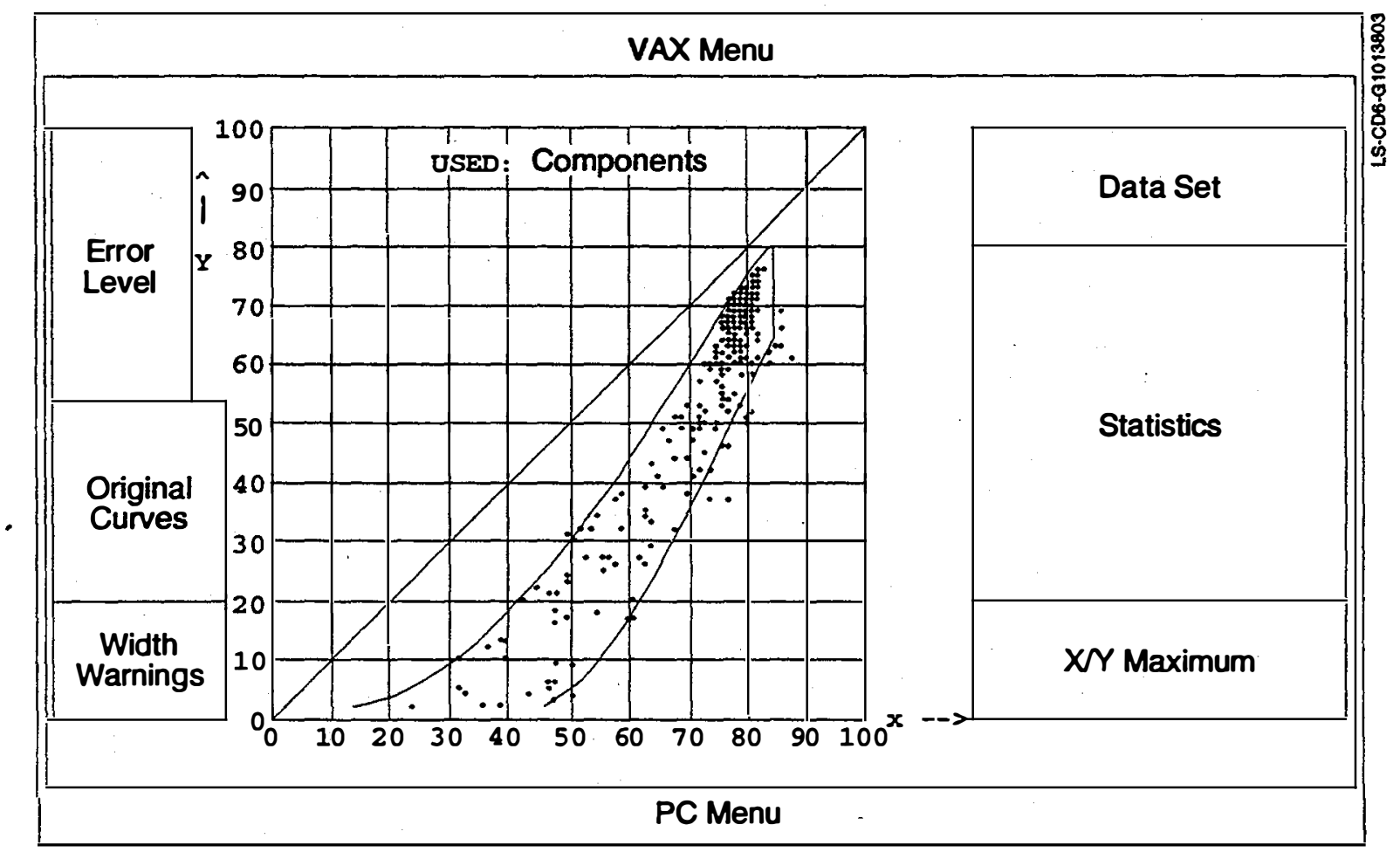

Figure 8-4. QCFIT screen information boxes

\subsubsection{Understanding the QCFIT Screen}

In addition to the main scatter plot display in the middle of the screen, several other display areas are defined on the screen, as shown in Figure 8-4.

For clarification, each area is shown with boxes in the figure, but the boxes do not appear on the computer screen. Each box in the figure is explained below:

The Menu Box contains menus and messages that prompt you for keyboard input during editing.

The Components Box displays the two components used to create the scatter plot. Although the scatter plot is based on $\mathrm{Kt}$ and $\mathrm{Kn}$, if either global horizontal or direct normal data are not available, the missing component is derived from the diffuse horizontal and the other available component.

The Error Level Box displays the relative error criteria used by the automatic fitting algorithm. It also shows the number of steps specified in the Wide and Narrow commands.

The Original Curves Box displays the source of the curves that QCFIT uses to display the initial scatter plot. The source can be from the QC-zero file, the 
automatic fitting algorithm, or the default curves (used if the automatic fitting algorithm lacks sufficient data to make an intelligent fit).

The Width Warnings Box displays warnings about the width of the selected boundaries. If this warning appears, the distance between the right and left Gompertz boundaries is abnormal for hourly data (too wide or too narrow, as indicated by the warning).

The Data Set Box displays the name of the input data file name, the site identifier, the month, and air mass regime.

The Statistics Box shows the selected curves and the percent of data points each exclude. The display also shows the total number of data points from the data file, the number (and percent) of points excluded, and the number of points ignored by QCFIT.

The XYY Max Box displays the Xmax and Ymax values selected for the Low air mass regime. It also shows the computed maximum values when editing Medium and High air mass data. These $\mathrm{X}$ and $\mathrm{Y}$ values are the integer percent representations of the actual $\mathrm{K}$-space values. For example, an $\mathrm{X} \max$ value of 82 represents a Kt value of 0.82 .

The format for the $\mathrm{X}$ or $\mathrm{Y}$ maximum in the box is as follows:

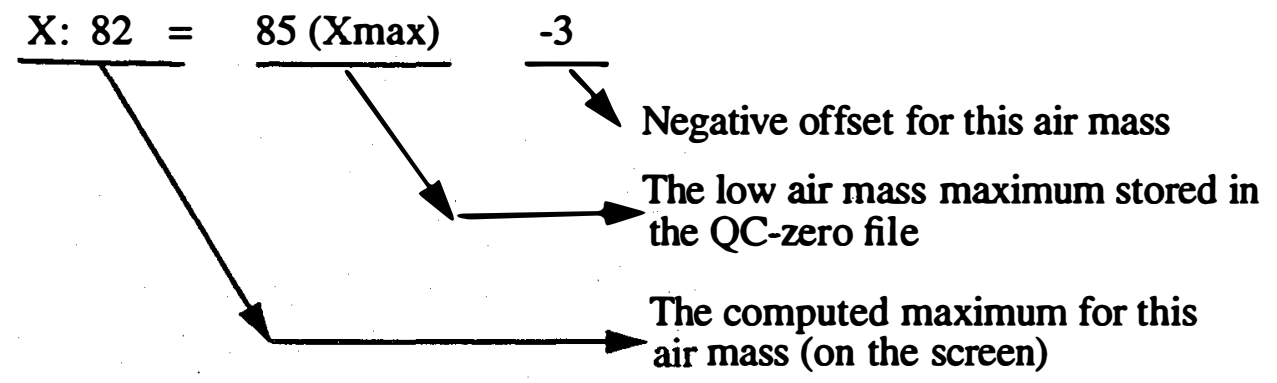

\subsubsection{Understanding the Scatter Plot Display}

Data points outside the boundaries are highlighted (bright on monochrome monitors and white on color monitors). Data points above the diagonal, which are ignored, are smaller (and in blue on color monitors).

If the data set contains many good, uniformly distributed points and few aberrant points, QCFIT does an excellent job of fitting curves to the data. If, however, the data set contains few data points, or points clustered in a small region, or many questionable points, you will likely have to edit the curves based on experience with boundaries and known information about the site. Sections 8.5.1 through 8.5.5 offer information that will help you choose boundaries for the scatter plot. 
Most data sets have a great number of values for which the direct normal measurement was near zero. Because these values are so common, their preponderance skews the automatic curve fitting algorithm. Thus, data points with a Kn component $<0.02$ are excluded from consideration by QCFIT. Although such data very likely occur in your data set, QCFIT neither displays the data nor uses it for automatic curve fitting.

\subsubsection{Choosing Menu Box Editing Options}

This section explains each of the editing options that appear in the Menu Box. Because boundaries for different integration times share some common information, some of the options will not work if curves have already been defined for other integration times.

Choose each editing option by pressing the letter in parenthesis, then $<\mathrm{CR}\rangle$.

\section{(S)elect}

The select option allows you to specify any of the four scatter plot boundaries (right and left Gompertz curves, and $\mathrm{Kt}_{\max }$ and $\mathrm{Kn}_{\max }$ ). You may invoke this option when it appears in the Prompt Box by entering:

$$
S<C R>
$$

QCFIT prompts you for each of four parameters in the order below.

\section{Left and Right}

(Note: The prompts and responses are nearly identical for specifying the left and right boundaries. Although you see \{LEFT,RIGHT \} in these instructions, only "LEFT" or "RIGHT" appears in the prompt on the screen.)

In the Prompt Box, QCFIT displays:

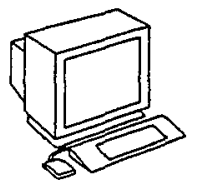

$$
\text { The \{LEFT, RIGHT\} side? [Y] }
$$

If you wish to select a different left-hand or right-hand Gompertz curve, enter Yes.

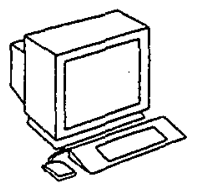

$$
\begin{aligned}
& \text { Please enter the curve type and position: } \\
& {[x, x]}
\end{aligned}
$$


Enter a pair of numbers that specify a new boundary. The first number specifies the curve shape and the second number specifies the curve position. (In Chapter 5, see Figures 5-2, 5-3, and 5-4, pages 36 and 37). For example, to specify curve 4, position 10, enter:

$$
4,10<\mathrm{CR}>
$$

If you mistakenly choose to change the boundary and wish to retain the current shape and position, simply enter:

$$
<\mathrm{CR}>
$$

If the numbers you enter specify a nonexistent curve, you have the opportunity to reenter the values after seeing this error message:

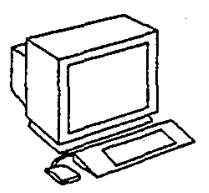

$$
\begin{aligned}
& \star \star \star \text { BOGUS CHOICE } \star \star \star \\
& \text { The }\{\text { LEFT, RIGHT }\} \text { side? [Y] }
\end{aligned}
$$

If boundaries have previously been established based on other integration times and you attempt to change the curve shape for the left or right boundary, you see the warning:

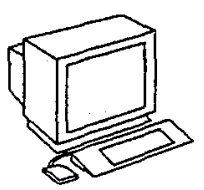

$$
\begin{aligned}
& \star \star \star \text { WARNING: Shape \#xx and position \#xx define } \\
& \text { the }\{\text { LEFT, RIGHT }\} \text { boundary for <integration } \\
& \text { time> data. OK to change? }(\mathrm{Y} / \mathrm{N})
\end{aligned}
$$

Answer No if you do not wish to change boundary definitions for data with the listed integration times. If you answer Yes, the selected boundary will also define the corresponding left- or right-hand boundary for other integration times.

Because QCFIT uses different right-hand position descriptors for different integration times, you may select a different right-hand curve position without affecting the other integration times. The left-hand boundary shares both shape and position with other integration times.

\section{$X \max$ and $Y \max$}

(Note: The prompts and responses are nearly identical for specif ying Xmax and $Y \max$. Although you see $\{\mathrm{X} \max , \mathrm{Ymax}\}$ in these instructions, only "Xmax" or "Ymax" appears in the prompts on the screen.)

$\mathrm{Xmax}$ and Ymax are integer percent representations of the upper limits on valid $\mathrm{Kt}$ and $\mathrm{Kn}$ values, respectively, for the low air mass data. The maximum for other air mass regimes is calculated from the low air mass maximum and is displayed in the 
lower right corner of the screen. (See Section 4.2.4 in Chapter 4, page 28, and Section 6.2 in Chapter 6, page 42, for more information about this calculation.) Thus, you may only specify the low air mass maximums. See "The X/Y Max Box" description in Section 8.4.1 for an explanation of the screen information.

In the Prompt Box you see:

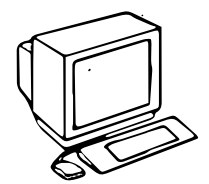

$$
\{X \max , Y \max \} ?[Y]
$$

Answer Yes if you want to change the value for the maximum. Answer No if you want to leave the current value unchanged. If you answer Yes, you see this prompt:

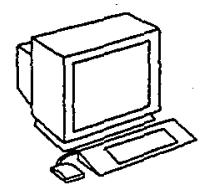

$$
\text { What value should it be? [xx] }
$$

Enter a number between 1 and 99 . If you decide to leave the value unchanged, enter:

$<\mathrm{CR}>$

If boundaries already have been defined based on data of other integration times and you attempt to change the Ymax value, you see the following message:
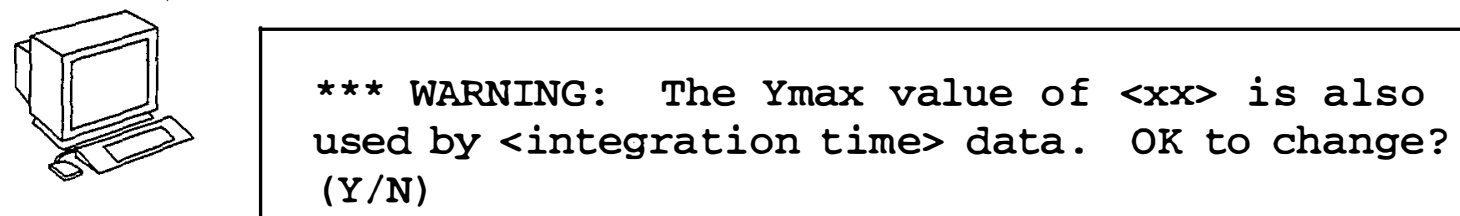

If you do not wish to change the Ymax already defined for other integration times, answer No. If you answer Yes, the new value will also define the Ymax for the integration times listed.

\section{(W)ide and (N)arrow}

These options allow you to move a boundary in increments of one position $(0.025$ $\mathrm{Kt}$ ). The Wide option moves a boundary away from the center of the scatter plot; the Narrow option moves a boundary toward the center of the scatter plot. You may select either option when they appear in the Prompt Box by entering:

$\mathrm{W}<\mathrm{CR}>$ (for the Wide option)

$\mathrm{N}<\mathrm{CR}>$ (for the Narrow option) 
QCFIT then prompts:

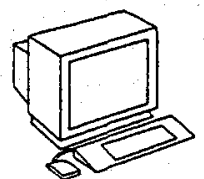

The LEFT side? [Y]

If you wish to move the left curve by one position, enter Yes or $<\mathrm{CR}>$. If you wish to leave the left curve in the same position, enter No. QCFIT then prompts:

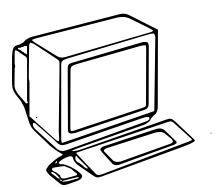

The RIGHT side? [Y]

If you wish to move the right curve by one position, enter Yes or $<\mathrm{CR}>$. If you wish to leave the right curve in the same position, enter No.

If boundaries have previously been established based on other integration times you may not use the wide or narrow options to move the left boundary. Because QCFIT uses different right-hand position descriptors for different integration times, you may change the right-hand curve position without affecting the other integration times. The left-hand boundary shares both shape and position with other integration times.

\section{(M)ore Error and (L)ess Error}

These options cause QCFIT to select curves using different criteria. The More Error option allows more data points outside the boundaries; the Less Error option requires more data points inside the boundaries. You may select either option when they appear in the Prompt Box by entering:

$\mathrm{M}<\mathrm{CR}>$ (for More Error)

$\mathrm{L}<\mathrm{CR}>$ (for Less Error)

If boundaries have previously been established based on other integration times and you attempt to use these options, only the position of the right-hand boundary will be considered by the fitting algorithm.

QCFIT selects curves based on the maximum "badness of fit" in the following manner:

Badness of fit $=$ (Fraction of excluded points) $\mathrm{x}$ Factor + (Relative accuracy of fit) / Factor

The curve minimizing the badness of fit is the one QCFIT selects. "Factor" is a weighting factor initially set to 1 ; every time More Error is selected, Factor is multiplied by 2; each time Less Error is selected, Factor is divided by 2 . Thus, the emphasis placed on lowering errors versus finding a desirable shape may be varied. 


\section{(D)elete Points}

PC The Delete Points option is not available on the PC version.

This option allows you to remove data points from consideration as QCFIT selects curves to fit the data.

Use this option with caution and only when you know a group of data is bad.

Data points that lie far from the general scatter of data have a significant influence on how QCFIT selects curves. If you know such data are bad (say, from a known instrument malfunction) or a questionable data point is unlikely for the stationmonth under observation, you may remove the point or points and allow QCFIT to select a more representative set of boundaries. Note that data above the diagonal line are automatically excluded from consideration by QCFIT. This deletion process in no way affects data in the input file.

You may select the Delete option when it appears in the Prompt Box by entering:

$$
\mathrm{D}<\mathrm{CR}>
$$

QCFIT prompts you for the vertices of a polygon to enclose the data points to be deleted.

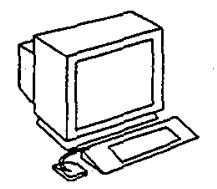

Vertex 1 =् X, Y coordinate:

Specify the first $X, Y$ coordinate of your polygon. Enter two numbers between 0 and 100 , separated by a comma, then enter $\angle C R>$. QCFIT responds by displaying a "@" character on the grid at the location specified, then prompts for confirmation:

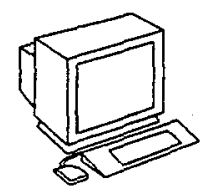

$$
\text { "Q" marks the spot? [Y] }
$$

If the coordinate is correct, enter Yes or $<\mathrm{CR}>$. If the coordinate is incorrect, you may correct it by entering No.

The program prompts for additional vertices:

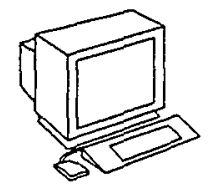

Vertex $2==>X, Y$ coordinate: 
Enter the X,Y coordinates of the second vertex. QCFIT displays a "@" character at the location specified, and prompts for verification:

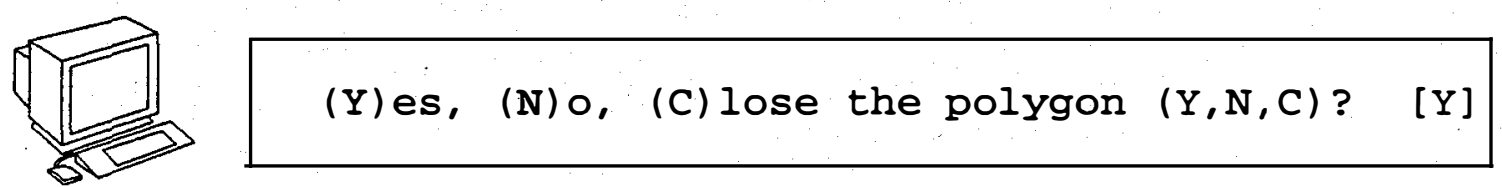

Respond with Yes or No as above. Continue specifying vertices as previously explained until the desired polygon is described. When all vertices have been specified, close the polygon by entering:

$$
\mathrm{C}<\mathrm{CR}>
$$

This closes the polygon and QCFIT prompts you to confirm the deletion:

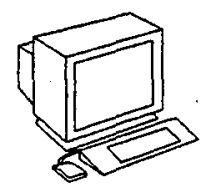

$$
\text { OK to delete }(\mathrm{Y}, \mathrm{N}) \text { ? [N] }
$$

To delete all the points in the polygon, enter Yes. To quit the delete process or start over, enter No or $<\mathrm{CR}>$.

As QCFIT deletes points, they are displayed as smaller dots on the screen (in blue on a color monitor) and are added to the count of points ignored. QCFIT then automatically reevaluates the curve fits and refreshes the screen.

If boundaries have been selected based on other integration times, the automatic fit algorithm is limited to the position of the right-hand boundary.

\section{(P)rint Screen}

VAX For the VAX, this option requires an attached DEC LA100 or compatible printer for the terminal you are using. To print the screen image using the attached printer, enter:

\section{$\mathrm{P}<\mathrm{CR}>$}

QCFIT provides no capability to send the screen image to the system printer or to any device other than an attached terminal printer. 
PC For the PC, this option requires that you have a PostScript printer attached to the computer's LPT1 port. (Optionally, you may specify a Hewlett-Packard LaserJet III or LaserJet 4 printer and specify another port or file for the printer output. See the instructions in Section 8.2.2.) To print the scatter plot, enter:

\section{$\mathrm{P}<\mathrm{CR}>$}

While the image is being printed, the screen clears and the following message appears:

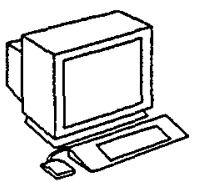

$$
\text { Please stand by ... }
$$

PC An alternate method of printing a screen image requires the use of MS-DOS 4.0 or 5.0 and a printer compatible with the MS-DOS GRAPHICS command. By issuing the GRAPHICS command with the appropriate parameters for your printer prior to running QCFIT, you may print the screen image by pressing the $<$ SHIFT > <PRINT SCREEN > keys on the computer keyboard. Consult your MS-DOS manual for more information about using the GRAPHICS command.

\section{(A)ir Mass}

This option allows you to examine and edit data in the other air mass regimes. To change the air mass specification, enter:

$\mathrm{A}<\mathrm{CR}>$

Before considering data from another air mass regime, QCFIT confirms the right and left Gompertz specifications for the current air mass regime:

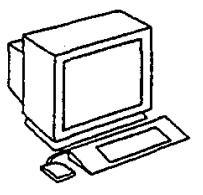

$$
\begin{aligned}
& \text { Okay to update file with } R: \quad\langle x x, x x>\text {; } L \\
& <x x, x \times>(Y, N) ?[Y]
\end{aligned}
$$

If you have specified or changed the right or left boundaries in the current air mass regime and wish to save them to the QC-zero file, enter Yes. If you have not made any changes, or do not wish to save the changes you made, enter No.

QCFIT prompts for the new air mass specification:

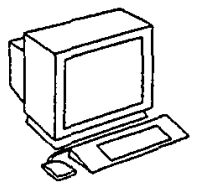

$$
\begin{aligned}
& \text { Which air mass regime, (L) ow, (M)edium, (H)igh } \\
& \text { [ ] }
\end{aligned}
$$


Specify the new air mass regime by entering $L, M$, or $H$, followed by a $<C R>$. You may stay with the current air mass regime (which appears in the brackets) by entering $\langle\mathrm{CR}\rangle$.

After you select the air mass regime, QCFIT plots the corresponding data and allows you to edit the curves with the editing options previously described.

\section{(Q)uit}

Use this option after specifying boundaries for all air mass regimes that could have data for the station-month. To quit, enter:

$$
\mathrm{Q}<\mathrm{CR}>
$$

QCFIT responds with the confirmation prompt for the curves specified in the current air mass regime:

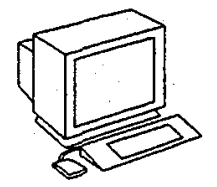

$$
\begin{aligned}
& \text { OK to update file with } R: \quad\langle x x, x x>\text {; }: \quad\langle x x, x x\rangle \\
& (Y, N) \text { ? }[Y]
\end{aligned}
$$

The values in the angle brackets indicate the current selections for the right and left boundaries. If you have specified or changed boundaries in the current air mass regime and wish to save them to the $\mathrm{QC}$-zero file, enter Yes or $<\mathrm{CR}>$. If you have not made any changes, or do not wish to save the changes you made, enter No.

QCFIT prompts for confirmation of $\mathrm{Kt}_{\max }$ and $\mathrm{Kn}_{\max }$ :

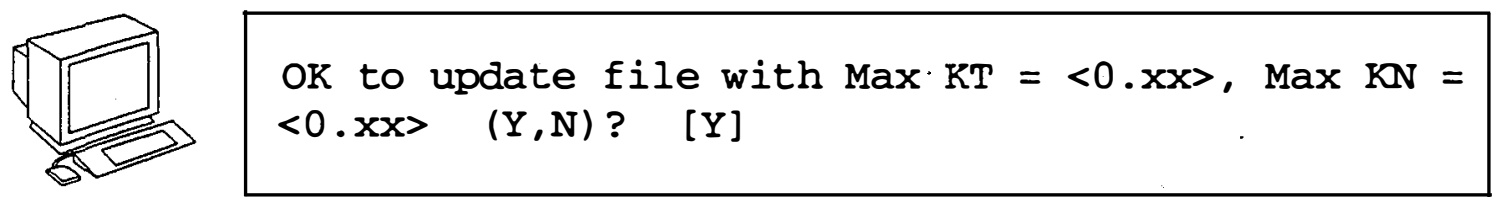

The values displayed in the angle brackets are the actual $\mathrm{K}$ values translated from the Xmax and Ymax integers you entered earlier. Remember, the displayed Max values are for the low air mass data; the values for the other air mass regimes are calculated. If you specified or changed the Xmax or Ymax values while editing any of the air mass regimes and wish to save these to the QC-zero file, enter Yes or $<\mathrm{CR}>$. If you made no changes or do not want to save your changes, enter No.

QCFIT prompts for continued operation:

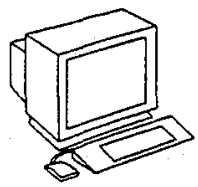

Do you wish to process another file? [Y] 
If you have finished examining data at this time, enter No.

VAX As part of the exit process, QCFIT purges previous versions of the QC-zero files, leaving only the most current version.

If you want to examine another station-month at this time, enter Yes or $<\mathrm{CR}>$.

QCFIT responds with:

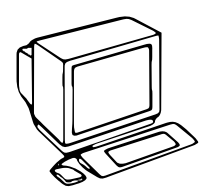

$$
\text { Is this site <site ID>? [Y] }
$$

If the data are from the same site (although not the same month) as the data just examined, enter Yes or $\langle C R>$. Continue processing with step 6 in Section 8.3 (Starting QCFIT).

If the data are from another site, enter No. Continue processing with step 4 in Section 8.3 (Starting QCFIT). 


\subsection{Evaluating the Boundaries}

Ideally, historical data from a monitoring station produce a well-defined envelope of data points on the QCFIT scatter plot, leaving no ambiguity when you select boundaries. In practice, however, several sources of error in data collection, as well as physical phenomena, affect the appearance of the scatter plot, making boundary selection difficult at times.

As a user, your goal is to select curves that represent a balance between Type 1 and Type 2 errors. Your boundaries should reject outliers (at the expense of rejecting good data), while including as much data as possible (at the expense of including bad data). As an aid, QCFIT reports errors for the right and left Gompertz boundaries independently, as well as an overall error rate for all boundaries. Experience shows that right and left boundary errors of between $2 \%$ and $5 \%$, and overall errors of between $5 \%$ and $15 \%$, meet this goal. The more data points and the less scatter of the data, the more comfortable you may be with lower error values.

A typical QCFIT scatter plot of good data shows a hysteresis-like envelope with boundaries snug against the bulk of the data and a few outliers close to the boundaries. Typically, the left-hand side of the scatter plot is more clearly defined than the right-hand side, and the right-hand side may have outliers at a greater distance from the boundary. In our experience using data of known good quality and of sufficient quantity, we have never found an envelope that is narrower at the bottom that at the top, or that pinches off at the top. By studying the QCFIT scatter plots and accompanying notes in the Appendix, you should get a better feel for acceptable envelope shapes, even when your data are sparse.

Remember that the Gompertz curve shapes are predetermined and may not always produce an excellent fit for data from all station-months. But experience shows that the typical discrepancy between the curve and the true data envelope rarely exceeds 3\% of ETR (full scale). Additionally, SERI_QC1 adds a buffer of 0.03 to the boundaries you select.

\subsubsection{Understanding the Effects of Air Mass}

In general, as the air mass increases from Low to Medium to High, the Gompertz boundaries move to the left (decreasing position numbers). On occasion, the position may remain unchanged, but any movement to the right (increasing position numbers) should be questioned.

The shape of the Gompertz boundaries may or may not change with air mass changes. If the shape of the right-hand boundary changes, one will usually note an increase in the slope with an increase in air mass. No such rule-of-thumb applies to shape changes for the left-hand boundary. 
Additional discussion of air mass effects will be found in Chapter 4, Section 4.2.4 (page 28).

\subsubsection{Considering the Effects of Climate and Season}

When evaluating curves, particularly for a small data set, consider the boundaries of adjacent months. Although sites usually exhibit seasonal differences, adjacent months often share the same curve shape and, less often, the same position.

When evaluating curves in this manner, you should always have a knowledge of a site's climate: Abrupt seasonal changes could lead to false conclusions about the expected envelope shapes based on adjacent months. An attempt should be made to explain envelope shapes in terms of the climate.

Additional discussion of climate and season effects will be found in Chapter 4, Section 4.2.4 (page 28).

\subsubsection{Assessing Data Density}

When evaluating large data sets, consider the density of data. QCFIT cannot display multiple occurrences of unique data points; a data point displayed on the screen may represent any number of similar measurements from the data file. As a result, when examining the plot, you may be inclined to give outliers more visual (or apparent) weight than they deserve. Under these circumstances, the error statistics reported by QCFIT are an invaluable aid in evaluating the fit of the boundaries.

The typical scatter plot rarely has uniform density. When selecting boundaries, give the greatest consideration to how the curves fit the more dense portions of the scatter plot.

\subsubsection{Previsualizing the Boundaries}

The effect of QCFIT's display can be deceiving: Even a poor fit can look good with the boundaries superimposed on the data. After examining enough plots to get a feel of how the Gompertz curves look, try this exercise: Visualize the boundaries before QCFIT draws the curves. Press the $<$ Pause $>$ or $<$ Hold Screen $>$ key immediately after the scatter plot appears but before the curves appear. Use a grease pencil to draw boundaries on the screen, keeping in mind the general shape of the Gompertz curves. Then press the $<$ Pause $>$ or $<$ Hold Screen $>$ key again to allow QCFIT to proceed, and compare your curves with those selected by the automatic fitting algorithm.

From this exercise, you gain not only a better understanding of the Gompertz shapes and limitations of the fitting algorithm, but also an appreciation of the illusion of fit created by the graphics image on the screen. You may find this 
exercise useful in the future when trying to decide between two or more curves, all of which seem to be appropriate fits for the data.

\subsubsection{Examples}

Users of QCFIT can benefit from a study of the Appendix, which contains screen prints of the final edited boundary fits for several stations that are part of NOAA's SOLRAD network. Only the months of January, April, July, and October are included. These stations represent a variety of climates in the United States and have been limited to those with good quality data. We hope these screen prints benefit you, and we invite you to send your own informative results and suggestions to be included in future editions of this manual. 


\subsection{Error Messages}

Several error messages may appear during operation of QCFIT. The following list explains the messages and how to remedy the problem that caused it.

*** UNKNOWN VARIABLE IN QCDEFLT.DAT

You have specified an unknown variable in the VARIABLES line of the QCDEFLT.DAT file. Check Table 8-2 in Section 8.2.2 for allowed variables, spelling, and capitalization.

\section{*** UNKNOWN INITIALIZER IN QCDEFLT.DAT}

You have specified an unknown variable in the INITIALIZE line in the QCDEFLT.DAT file. Check Table 8-2 in Section 8.2.2 for allowed variables, spelling, and capitalization. Check the INITIALIZATION instructions in Section 8.2.2 for the correct format.

\section{*** UNITS UNKNOWN IN QCDEFLT.DAT}

You have specified an unknown measurement unit code in the UNITS line in the QCDEFLT.DAT file. Check Table 8-3 in Section 8.2.2 for the allowed units codes.

\section{*** UNKNOWN SPECIFICATION IN QCDEFLT.DAT}

You have used an unknown defaults specification in the QCDEFLT.DAT file. Check the list in Section 8.2.2 for allowed defaults specifications.

The printer code specified in the printer line in the QCDEFLTDAT file was not recognized. Check the printer specifications in Section 8.2.2 for allowed printer codes.

Printer Driver Not Found: <DRIVER NAME>

The printer driver supplied with the PC version was not found in the logged directory when you attempted to use the (P)RINT COMMAND. Copy the driver to the directory from which QCFIT is being run. 


\subsection{QCFIT as a Diagnostic Tool}

QCFIT can be used as a very effective diagnostic tool to identify various problems encountered when measuring and processing solar radiation. July data collected by the University of Oregon at Eugene, OR, will be used to illustrate the various measurement problems that can occur. The original data plotted in Figures 8-5 and 8-6 for the low and high air mass ranges are of good quality and span a 13-year period of time. Almost 2,400 hourly values are included in these two plots. Various methods were employed to simulate problems that have been observed when processing other data. The medium air mass effects of the problems illustrated would be similar to those shown for low and high air masses.

\subsubsection{Understanding Tilt Effects}

Although pyranometers are equipped with a bubble level, it is not unusual to find that the sensor surface is not in the same plane as the level. Also, the structure on which a pyranometer has been mounted has been known to shift its position as a result of wind, temperature changes, etc. Furthermore, nonplanar sensor surfaces have been observed. Therefore, one cannot assume that the sensor of the pyranometer was in a perfectly level position when the solar radiation data were collected.

The effects of tilted sensors were simulated by calculating the direct beam irradiance on tilted sensors while assuming negligible change in the horizontal diff use element (a reasonable assumption for the small tilts employed). These modified data were then submitted to QCFIT. The boundaries selected for the original data were retained.

The effect of a sensor tilted $2^{\circ}$ to the south is shown in Figure 8-7 for the high air mass range. At low air mass (high solar elevation) the effects of a $2^{\circ}$ tilt to the south were not apparent. However, at high air mass we note that the dense cluster of data representing clear skies has moved closer to the diagonal. This is particularly noticeable for the data at the bottom of the dense cluster. Although this is not an extremely noticeable effect, it would be unusual for the dense cluster of data under clear sky conditions to virtually parallel the diagonal for the distance shown on Figure 8-7. This is the result of increased incidence angles during the early morning and late afternoon hours, when the July sun is north of the sensor.

Next, a tilt of $2^{\circ}$ to the east was simulated. Again, the effects of this tilt were not apparent for low air mass. At high air mass, however, the effect of a $2^{\circ}$ tilt to the east is very apparent. In Figure 8-8, we see that the dense cluster of data has now been split into three populations. It was originally expected that the data would be split into two populations, one abnormally high and one abnormally low, representing early morning and late afternoon positions of the sun. Actually, the three clusters represent data collected during three specific hours. The far right 


\section{Low}

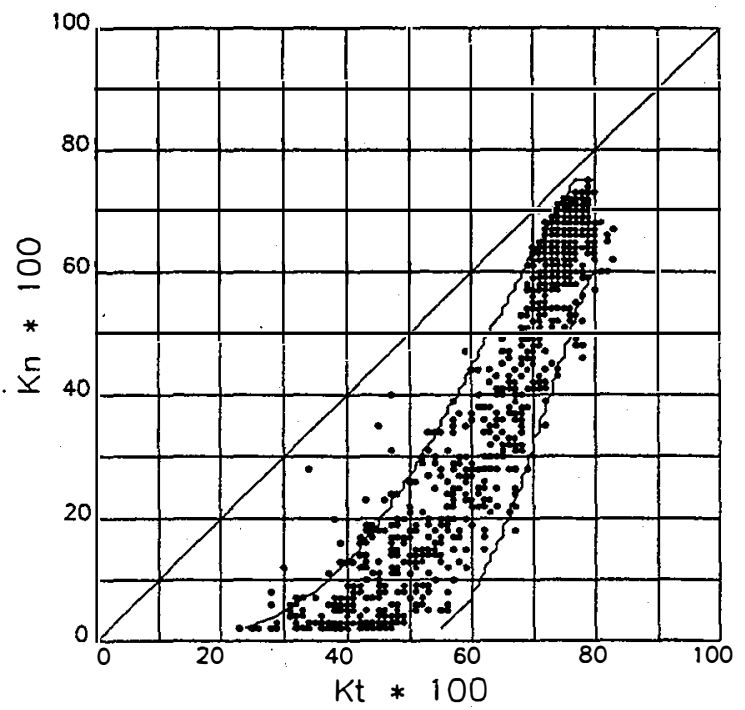

Figure 8-5. Scatter plot of original data for low air mass for July at Eugene, OR

High

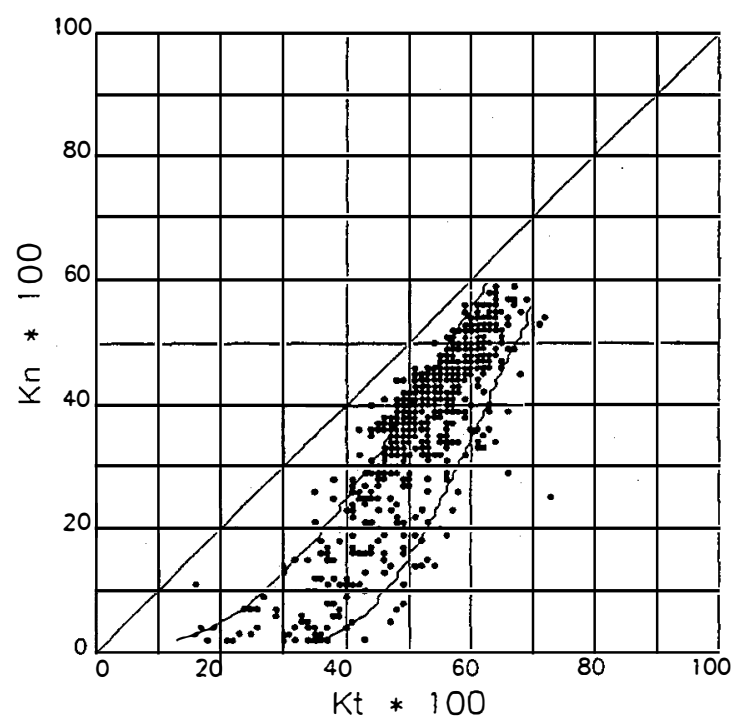

Figure 8-7. Scatter plot of data in Figure 8-6 modified to simulate a sensor tilted $2^{\circ}$ to the south
High

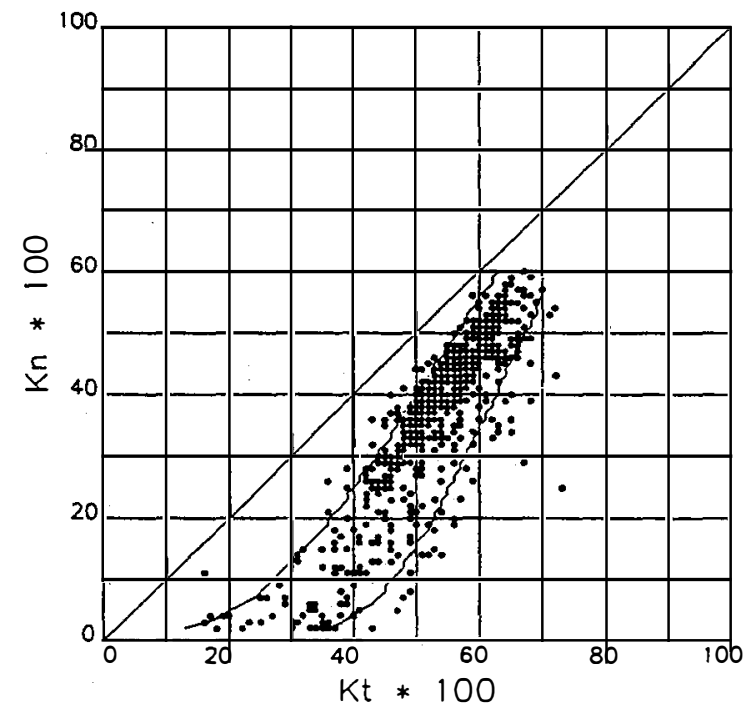

Figure 8-6. Scatter plot of original data for high air mass for July at Eugene, OR

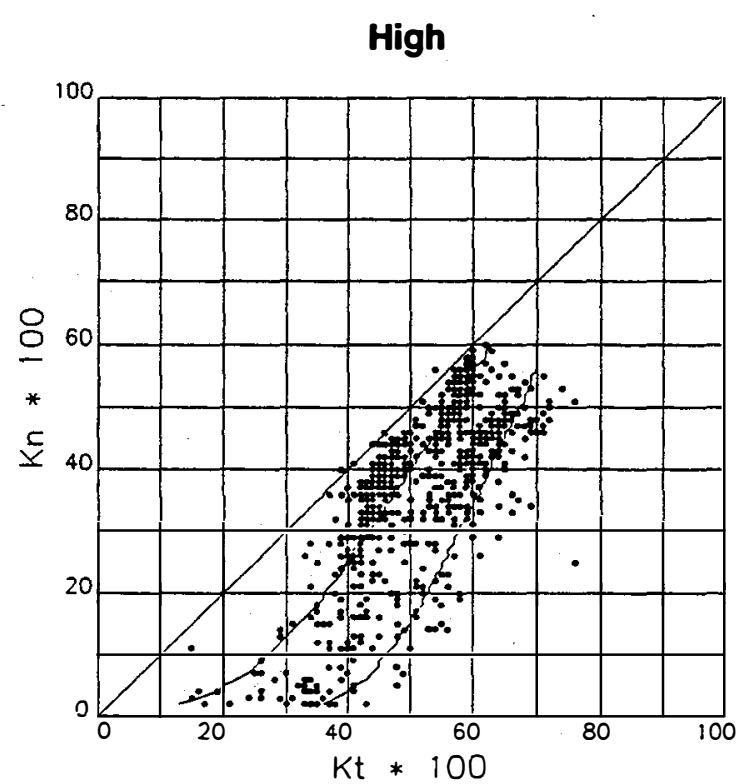

Figure 8-8. Scatter plot of data in Figure 8-6 modified to simulate a sensor tilted $2^{\circ}$ to the east 
cluster are data collected at 0700 in the moming, the middle cluster of data are from 1800 in the afternoon, and the left cluster was collected at 1900 (7 p.m.).

Figure 8-9 shows the low air mass effects of a sensor tilted $5^{\circ}$ to the south. When compared with Figure 8-5, the increase in global values is consistent with expectations, since the angle of incidence on the sensor surface has been reduced. It is not likely, however, that this effect would be noted during routine data processing. The effects of a $5^{\circ}$ south tilt are very noticeable at high air mass, as shown in Figure 8-10, where the global values have decreased, moving the data points closer to the diagonal. None of the Gompertz curves used by QCFIT will achieve a good fit to these data. A tilt to the north would displace the data a similar distance away from the diagonal, and although they might be fit by one of the Gompertz curves, the separation from the diagonal would be considerably greater than that found for good quality data.

Figure 8-11 shows the low air mass effects of a sensor tilted $5^{\circ}$ to the east. We now see that the dense cluster of data for high air mass conditions is significantly wider than the original data shown on Figure 8-5, with most of the spread moving toward the diagonal. The extra width would not of itself be a good diagnostic, since various atmospheric conditions (turbidity and water vapor) can produce a distribution similar to that seen in Figure 8-11. However, in no instance will good quality data be clustered that close to the diagonal. As will be noted later, this could also be the effect of a miscalibration of the pyranometer. However, a miscalibration of the pyranometer would not produce the effects seen in Figure 8-12 for high air mass. The splitting of the dense cluster of data into three distinct populations is now much more noticeable than it was with a $2^{\circ}$ tilt to the east. Also, two of these clusters are mostly above the diagonal, and one of the clusters is far to the right of the diagonal. Although the data were grouped into three air mass ranges because of the effects of atmospheric attenuation, this also increases the value of QCFIT as a diagnostic tool, albeit serendipitously.

\subsubsection{Recognizing Time and Position Errors}

The extraterrestrial solar radiation values used to normalize the original solar radiation data for presentation in $\mathrm{K}$-space is a function of the designated position of the sensor on the surface of the earth. Any error in the designated position will result in a movement of the data when plotted in K-space. Similarly, because time is a function of longitudinal position, any errors in time will also result in errors in the computation of extraterrestrial solar radiation. These errors will also be reflected in errors in the computation of $\mathrm{K}$-space values. 
Low

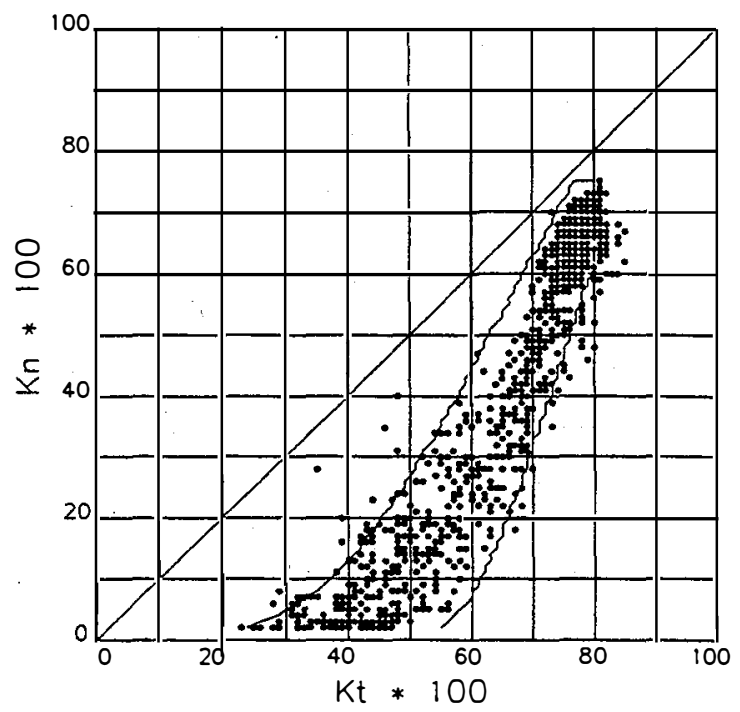

Figure 8-9. Scatter plot of data in Figure 8-5 modified to simulate a sensor tilted $5^{\circ}$ to the south
Scatter plot of data in Figure 8-6 modified to simulate a sensor tilted $5^{\circ}$ to the south

\section{Low}

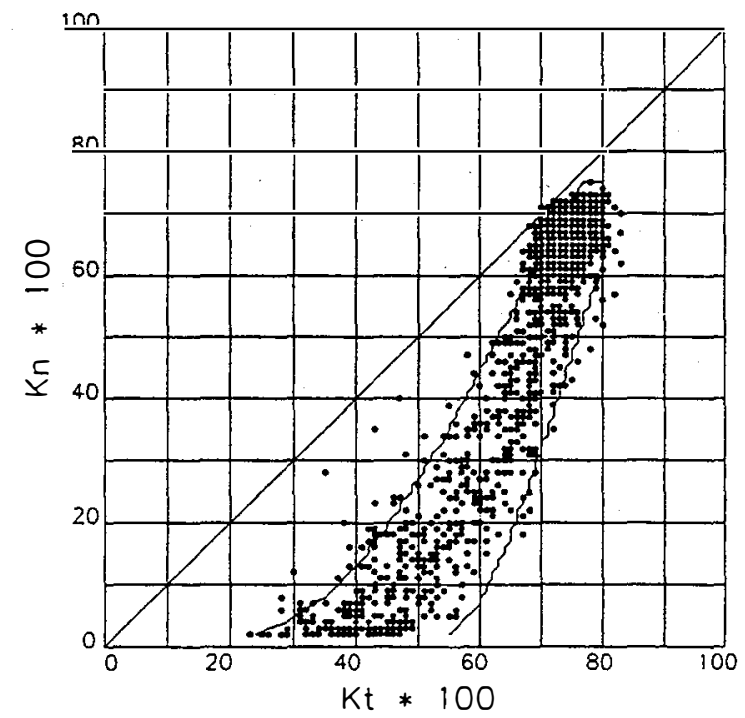

Figure 8-11. Scatter plot of data in Figure 8-5 modified to simulate a sensor tilted $5^{\circ}$ to the east
Figure 8-10.

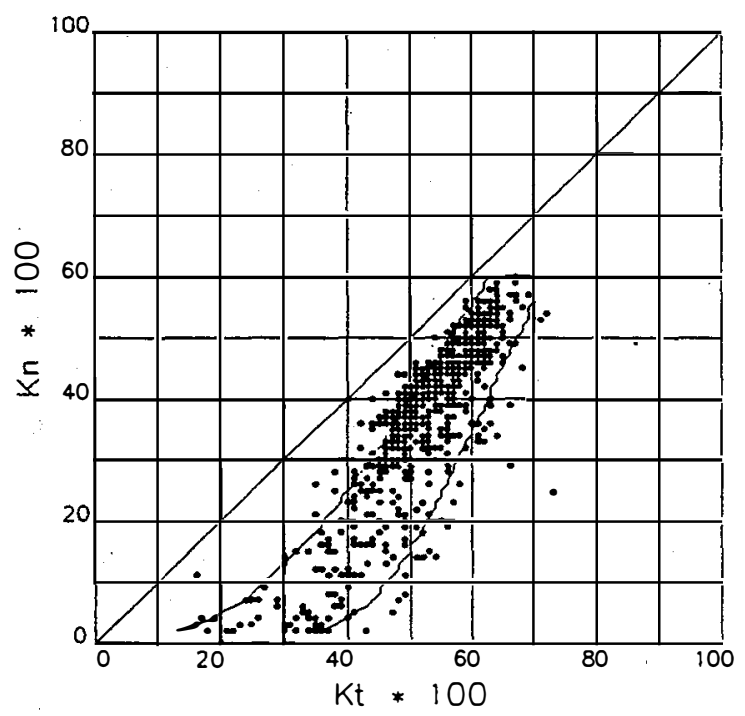

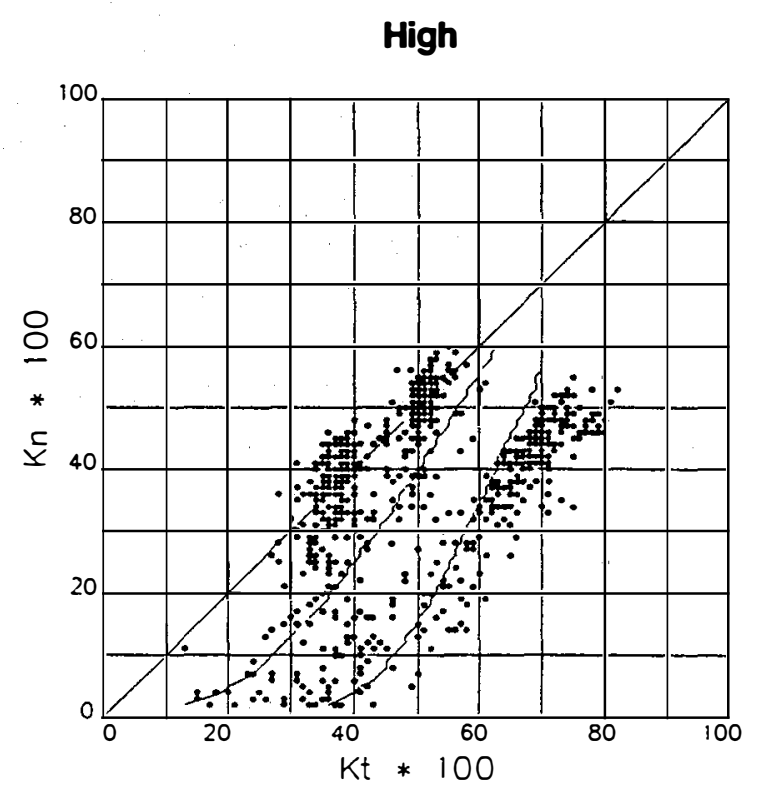

Figure 8-12. Scatter plot of data in Figure 8-6 modified to simulate a sensor tilted $5^{\circ}$ to the east

QCFIT Software Users Guide - 135 
Figure 8-13 shows the effects of a $1^{\circ}$ error in longitudinal position, which also represents the effects of a 4-minute error in time. These results are for the high air mass range; the results at low air mass were not significant. The effects of a $4^{\circ}$ error in longitude or a 16-minute error in time are shown in Figure 8-14 for the high air mass range. We see that these effects are similar but not identical to the effects of tilted sensor surfaces. Smaller effects will be seen at low and medium air mass ranges.

The effects of errors in position and time change the calculation of extraterrestrial radiation, rather than resulting in a change in the measured value, as was observed for tilt effects. Therefore, these effects are seen under both clear and cloudy skies. In other words, a wider dispersion of the data are observed in Figures 8-13 and 8-14 across the full range of $\mathrm{Kn}$ or direct normal values. The effects of tilted sensors, however, are most noticeable under clear skies when direct beam radiation from the solar disk dominates the irradiance at the surface of the earth.

Figures 8-15 and 8-16 show the low and high air mass effects of a $10^{\circ}$ shift in designated latitude. Again, this results in a change in the calculated extraterrestrial solar radiation and the effects are noted for all sky conditions (clear and cloudy). We see that for the low air mass range all of the data have been shifted to the left, closer to the diagonal. For the high air mass range, the data have been shifted to the right, farther from the diagonal, and have been dispersed across a wider range of Kt values.

For low air mass, the shifting of the data to the left, to lower Kt values, is a direct result of increased horizontal extraterrestrial solar radiation during the middle part of the day at the lower latitude. The effects for the high air mass range can be attributed primarily to the longer day observed at high latitudes during the summer. The high air mass values occur closer to solar noon at a latitude of 34.05 than at a latitude of 44.05. Therefore, the actual solar elevation at the higher latitude was higher than that calculated and the measured solar radiation was higher than would have been observed at that time of day at the lower latitude. This results in the high $\mathrm{Kt}$ values shown on Figure 8-16. The general increase in the dispersion of the data along the $\mathrm{Kt}$ axis results from different effects in the morning and evening and changing effects during the month, because the changes in day length at the more northem location are more pronounced than at the southern location. 
High

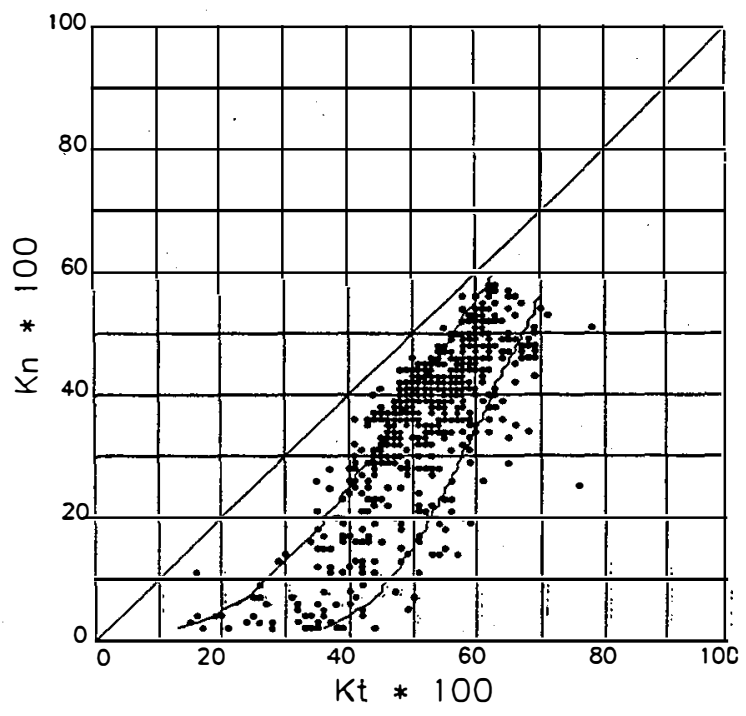

Figure 8-13. Scatter plot of data in Figure 8-6 modified to simulate a $1^{\circ}$ error in longitude or a 4 minute error in time

\section{Low}

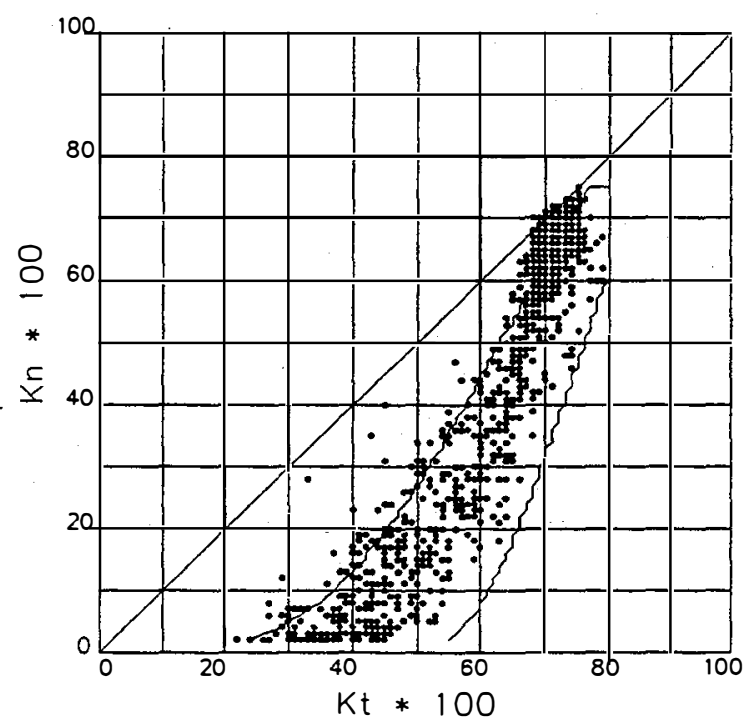

Figure 8-15. Scatter plot of data from Figure 8-5 modified to simulate a $10^{\circ}$ error in latitude
High

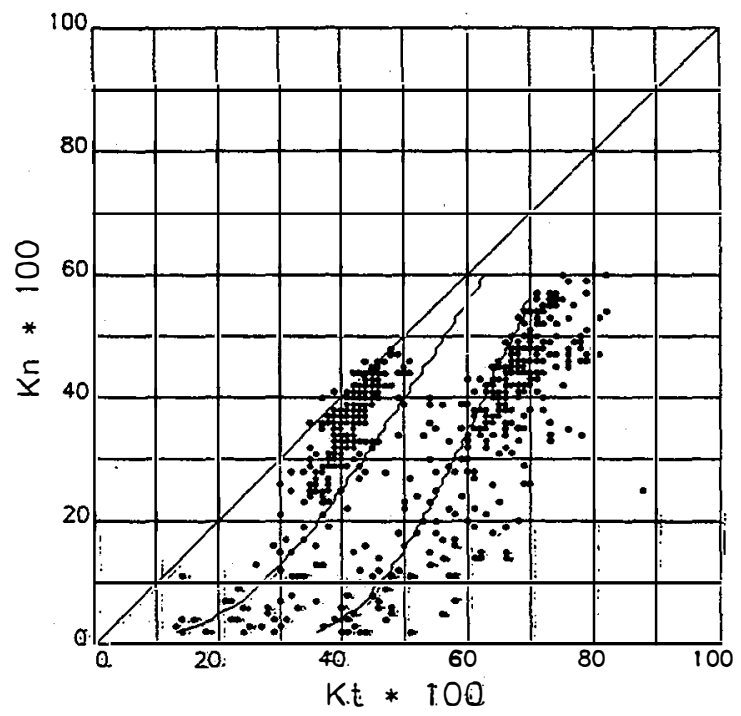

Figure 8-14. Scatter plot of data in Figure 8-6 modified to simulate a $4^{\circ}$ error in longitude or a 16minute error in time

High

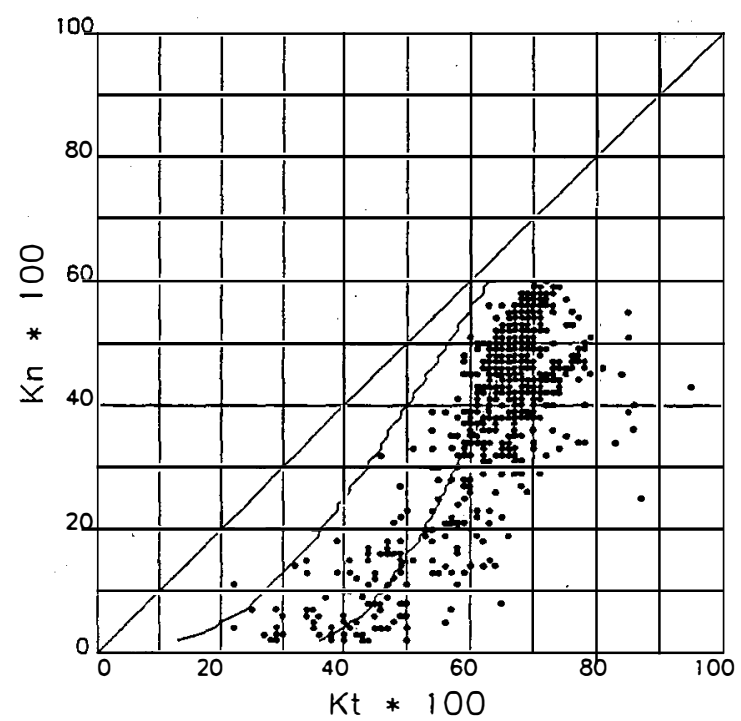

Figure 8-16. Scatter plot of data from Figure 8-6 modified to simulate a $10^{\circ}$ error in latitude 


\subsubsection{Detecting Calibration Errors}

The possible combinations of calibration errors are enumerable, especially when dealing with data using three instruments, each of which can be miscalibrated either high or low. Even for two-element data, there are a number of combinations, each of which will produce different effects in K-space. We will show just a few of these possible combinations to illustrate the various effects that might be observed.

Figure 8-17 shows the effects of miscalibrating the pyranometer by minus 5\%. Because the pyranometer has been given a calibration factor indicating a lower than actual sensitivity, the apparent global horizontal solar radiation is $5 \%$ high. The 5\% error is greatest at high levels of solar radiation, hence the upper part of the scatter plot is shifted to the right more than the bottom part. There is no significant change in the dispersion of the data. The primary indication of this error is given by the separation between the diagonal and the dense cluster of data representing clear sky conditions. For the low air mass range, the separation shown on Figure 8-17 is at least unusual if not abnormal. Similar but somewhat less noticeable changes were observed for the medium and high air mass ranges.

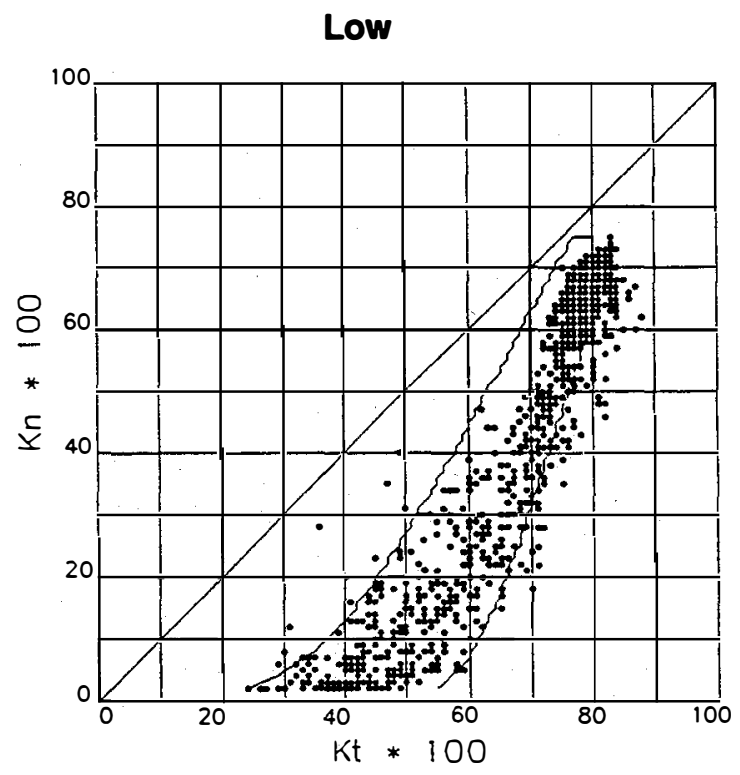

Figure 8-17. Scatter plot of data in Figure 8-5 modified to simulate the effects of miscalibrating the pyranometer by minus $5 \%$ 
Figure 8-18 shows the effects of a 5\% error in the opposite direction. These results are almost identical to those shown on Figure 8-15 for a $10^{\circ}$ error in the latitude assigned to the station. However, the results shown on Figure 8-15 are due to the calculation of high extraterrestrial values whereas the results shown on Figure 8-18 are due to the assignment of low surface values resulting from a high calibration factor.

In Figure 8-19 we see a very similar effect resulting from the assignment of a 5\% low calibration factor to the pyrheliometer used to measure direct normal solar radiation. In this instance, the upper portion of the scatter plot is crowding the diagonal because of high $\mathrm{Kn}$ values rather than low $\mathrm{Kt}$ values. The results shown in Figure 8-20 indicate that a 5\% error in the calibration of the pyrheliometer would be very difficult to detect at the high air mass range.

Figure 8-21 shows the results of assigning 5\% low calibration factors to both the pyranometer and the pyrheliometer. These data look completely normal in every respect, and it would be extremely difficult to detect any problems. This type of error could result from errors in calibrating a reference standard used in the calibration of both field instruments. Similar problems could occur when using a single pyranometer to measure both global and diffuse radiation. When a single instrument is used to measure more than one solar radiation element, there is an effective loss in the degrees of freedom. Of course, when calibration errors in opposite directions occur, the effects will be very noticeable. This is illustrated in Figure 8-22, which simulates a 5\% low calibration for the pyranometer and a 5\% high calibration for the pyrheliometer. The result at the upper end of the scatter plot is a large separation between the data and the diagonal. Although the errors are only $5 \%$, the effect is multiplied to give the appearance of a much larger error.

\subsubsection{Detecting Misadjustment of Shadowbands}

The high cost of solar trackers forces many stations to measure only the global horizontal and horizontal diffuse elements. As noted in Chapter 2, the most common instrument for measuring diffuse horizontal radiation is a pyranometer with a shadowband. The position of the shadowband must be adjusted every day or two as the solar declination changes. If the shadowband is not adjusted or is misadjusted, a portion of the sensor may be irradiated by the direct beam, resulting in an abnormally high diffuse value. When the global horizontal and diffuse elements are used to calculate the direct normal element, the high diffuse values result in an apparent low direct normal value. This effect is shown on Figure 8-23 for data obtained at Albuquerque, NM, during the month of June. The actual global and direct normal data are shown in Figure 8-24 for comparative purposes.

\subsubsection{Recognizing the Effects of Snow, Frost, and Dirt}

At most locations, there is a possibility that the globes or windows covering the sensing elements of the radiometers may become contaminated by elements such as snow, frost, dirt, and raindrops. This contamination of the globes and windows 
Low

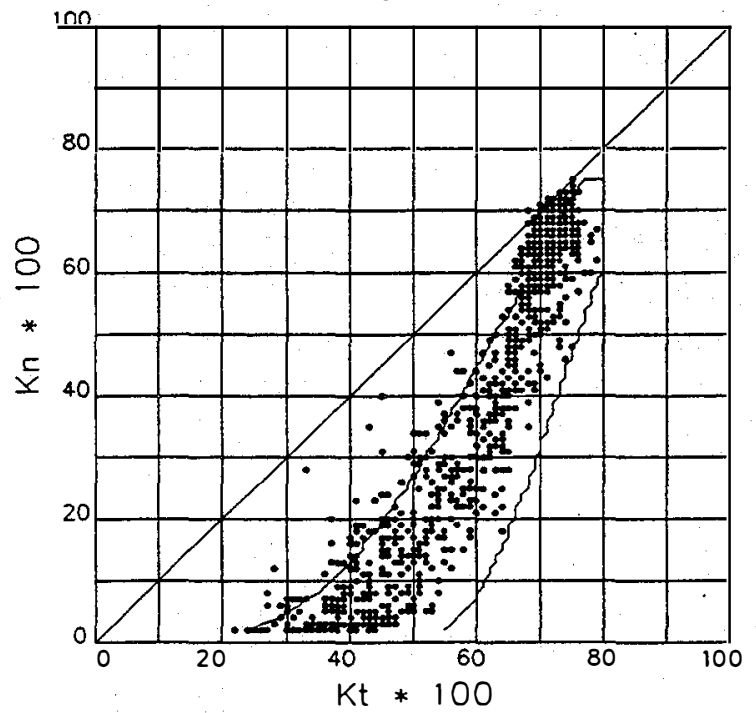

Figure 8-18. Scatter plot of data in Figure 8-5 modified to simulate the effects of miscalibrating the pyranometer by plus $5 \%$

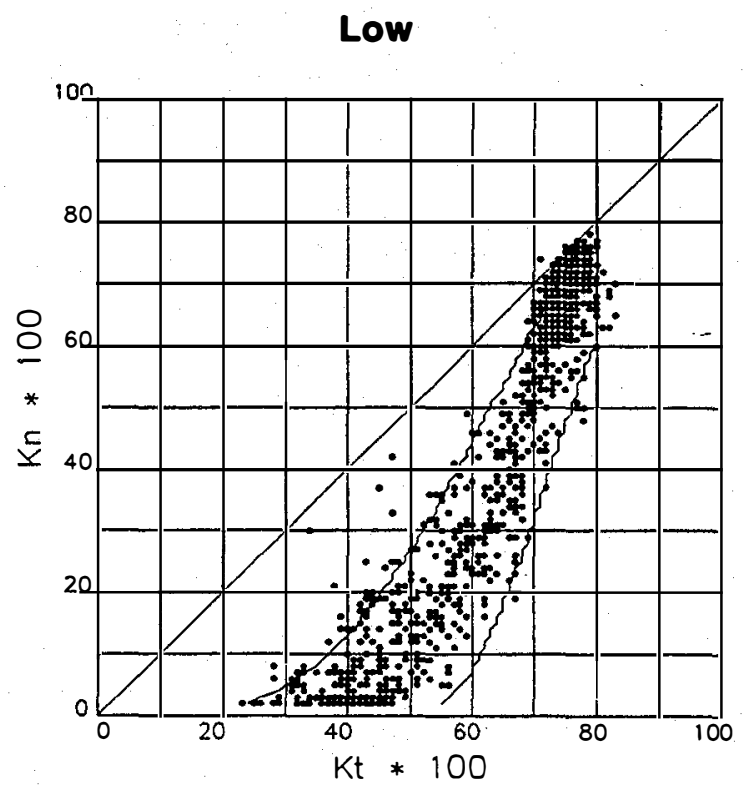

Figure 8-19. Scatter plot of data in Figure 8-5 modified to simulate the effects of miscalibrating the pyrheliometer by minus $5 \%$
High

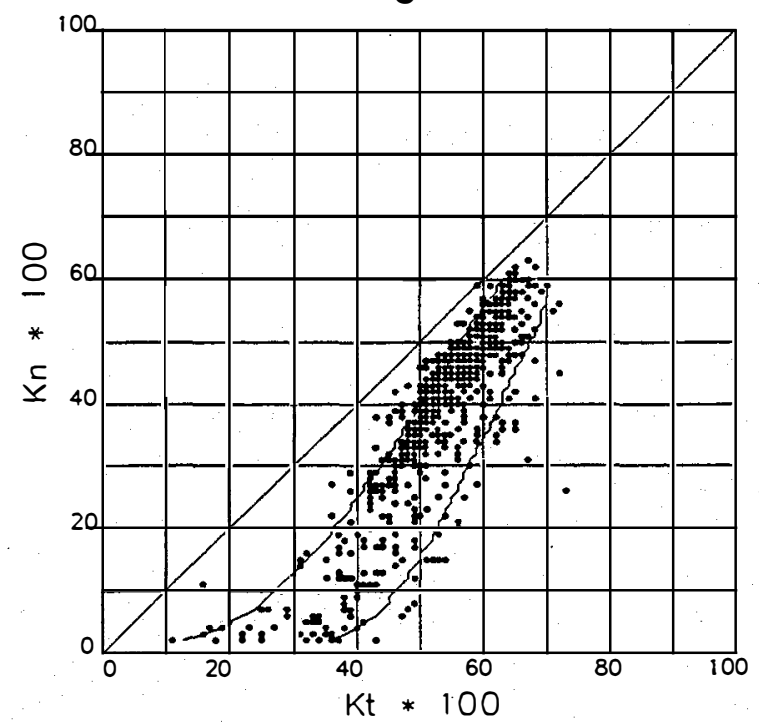

Figure 8-20. Scatter plot of data in Figure 8-6 modified to simulate the effects of miscalibrating the pyrheliometer by minus 5\%

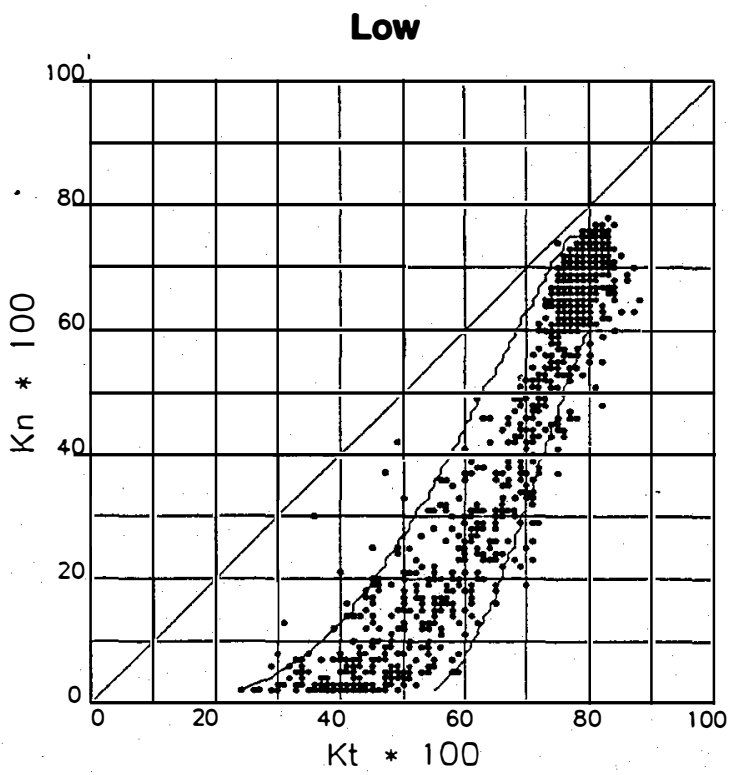

Figure 8-21. Scatter plot of data in Figure 8-5 modified to simulate miscalibrating both the pyranometer and pyrheliometer by minus $5 \%$ 


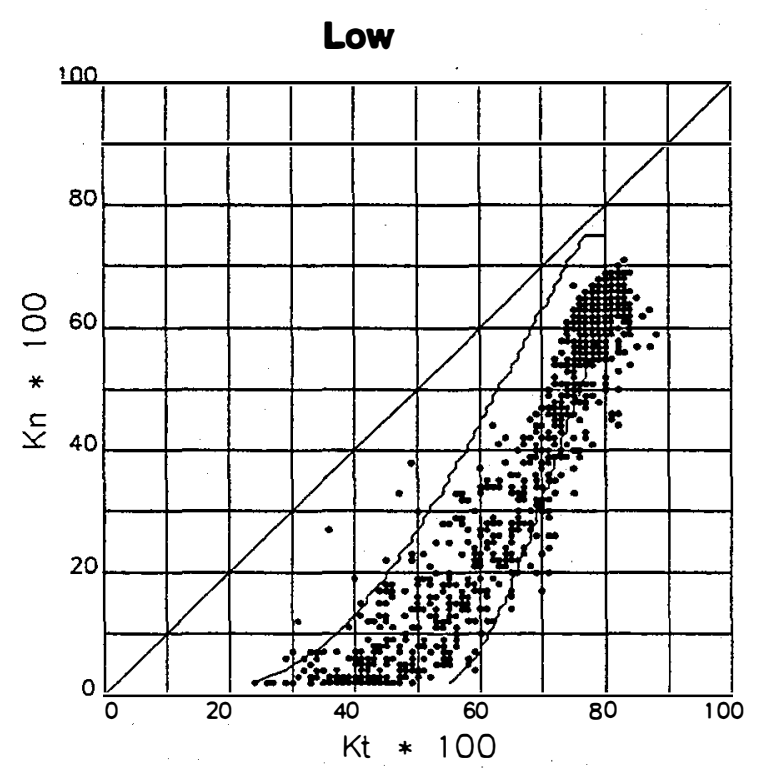

Figure 8-22. Scatter plot of data in Figure 8-5 modified to simulate miscalibrating the pyranometer by minus 5\% and the pyrheliometer by plus $5 \%$

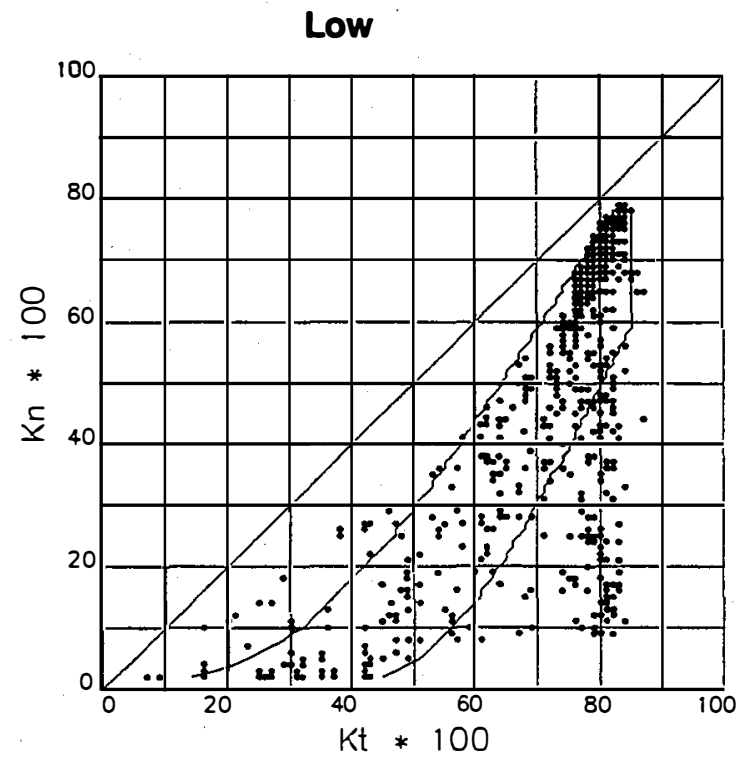

Figure 8-23. Scatter plot of global-diffuse data for low air mass for June at Albuquerque modified to simulate a bad shadowband adjustment

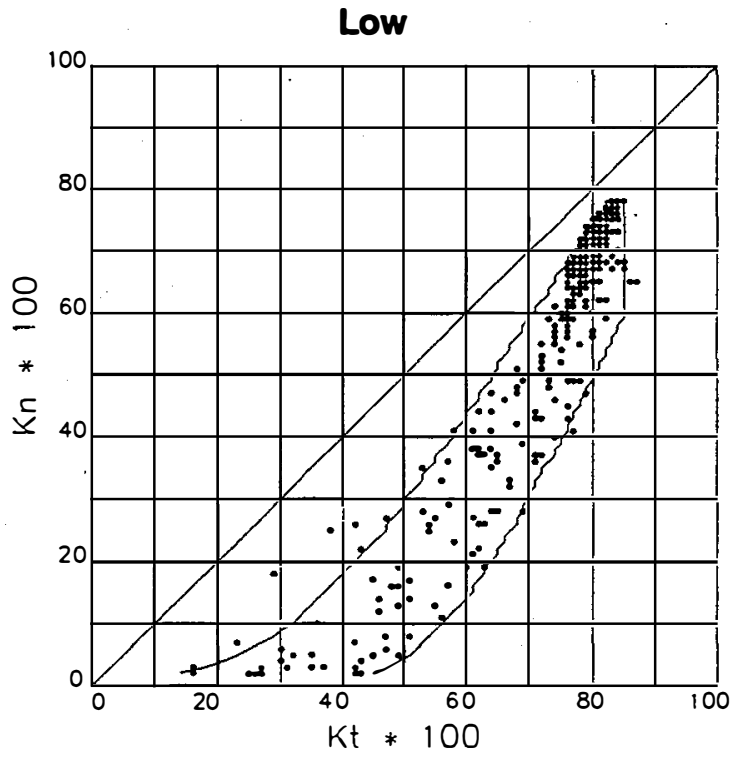

Figure 8-24. Scatter plot of unmodified globaldirect data for Albuquerque shown in Figure 8-23 
will result in abnormal solar radiation measurements, which may be detectable when viewed on QCFIT scatter plots. Figure 8-25 shows data collected by Bluefield State College, Bluefield, WV, during the month of November. These data are for the high air mass range.

It is apparent that both abnormally high and abnormally low global horizontal data are displayed on this figure. We cannot be sure of the factors that produced these anomalous data. Nevertheless, from an examination of the maintenance records for the Bluefield State station, there is good reason to believe that the abnormally low values were the result of snow covering the pyranometer globes, and that the abnormally high values are the result of frost on these globes. Snow, of course, tends to block the solar radiation from reaching the pyranometer. However, frost tends to diffuse the direct beam radiation, and at high zenith angles this can increase the irradiance of the sensor.

We do know that the abnormally low global horizontal data occurred on a weekend following a Friday snow storm. There was no maintenance on the weekend; i.e., cleaning of the globes. Similarly, the abnormally high global horizontal values occurred early in the morning under clear sky conditions when frost could have been present and when the direct beam radiation would have been high. These suppositions are supported by Figure 8-26, for which the direct normal values were calculated from the diffuse values. We see that the combination of abnormally high global horizontal values and probably somewhat low diffuse horizontal values (the shadowband prevents the irradiance of the diffuse pyranometer by the direct beam) result in abnormally high direct normal estimates.

We also note that the data points above the diagonal on Figure 8-25 have been pulled down very close to the left boundary on Figure 8-26. This would be the result of the calculation of direct normal values from low global horizontal and low horizontal diffuse values. It should be noted that experience has shown that snow and frost are much more likely to collect on the pyranometer globe than on the pyrheliometer window. 
High

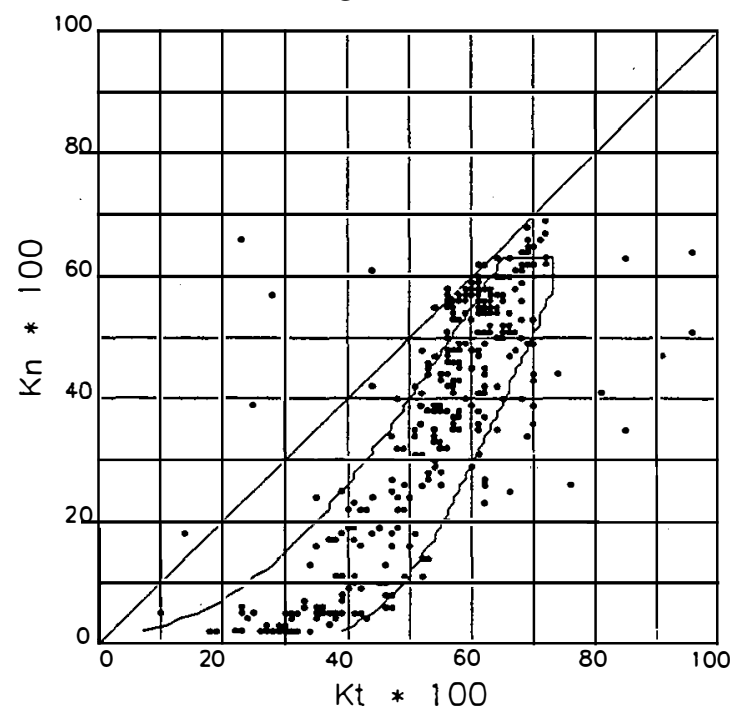

Figure 8-25. Scatter plot of global-direct data for high air mass for November at Bluefield, WV. The widely scattered data points are probably due to effects of snow and frost.
High

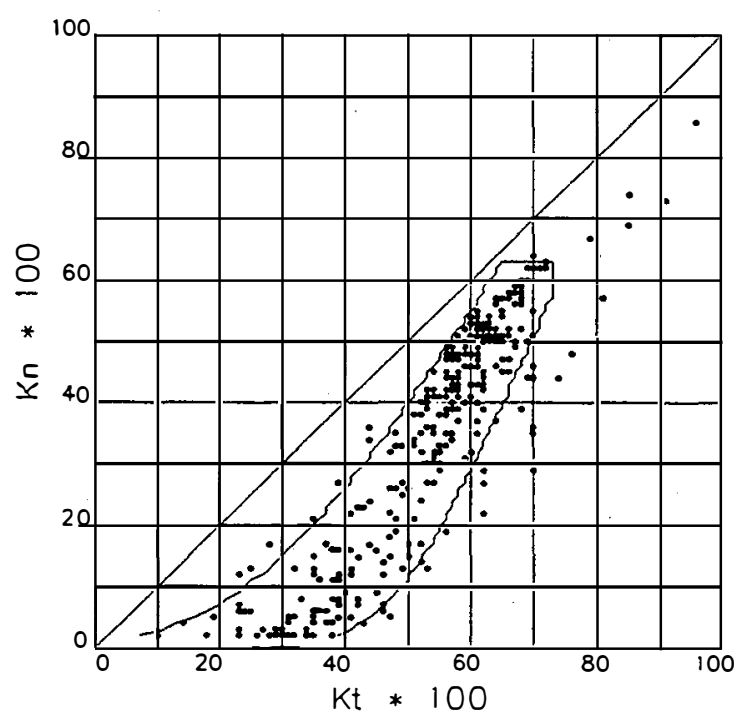

Figure 8-26. Scatter plot of global-diffuse data for high air mass for November at Bluefield, WV. A comparison of this figure with Figure 8-25 adds support to the supposition that these data were affected by snow and frost. 



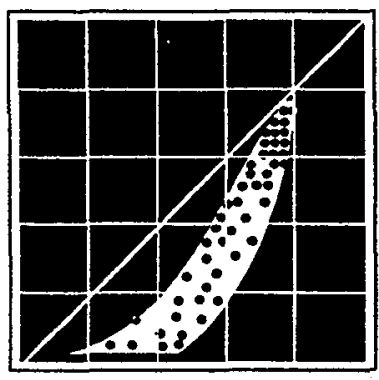

\section{Chapter 9}

\section{SERI_QC1 Software Users Guide}

\section{Contents}

9.1 Input and Output Requirements...................................................... 146

9.1.1 Specifying Inputs ............................................................. 146

9.1.2 Understanding Outputs ....................................................... 147

9.2 A Sample Calling Program .............................................................. 148

9.2.1 Comments on the Sample Program ...................................... 149

9.2.2 Writing Your Own Calling Program ....................................... 151

9.3 SERI_QC1 Subroutine Description ................................................... 153

9.3.1 Validating the Inputs .............................................................. 153

9.3.2 Trivial Category Tests .......................................................... 155

9.3.3 One-Component Tests .......................................................... 156

9.3.4 High-Direct Test ................................................................... 156

9.3.5 Three-Component Tests .......................................................... 156

9.3.6 Two-Component Tests ............................................................ 156

9.4 SERI_QC1 Program Notes ............................................................ 159

\section{Overview}

The SERI_QC1 subroutine accesses the quality of solar irradiance data using station-specific information contained in QC-zero files. (See Chapter 7, Section 7.3, page 87, for a description of these files.) The subroutine is part of a solar radiation quality assessment software package that includes the program QCFIT, which creates the QC-zero file used by SERI_QCl.

SERI_QC1 accepts solar irradiance and other data from a calling program and returns a two-digit flag that provides information on the quality of each of the measured solar radiation components. 


\subsection{Input and Output Requirements}

The SERI_QC1 subroutine calling format, which includes both input and output parameters, is as follows:

CALL SERI_QC1 ( Site, Iyear, Month, Iday, Ihour,

2 Minute, Intrvl, Global, Direct, Difuse, IQCglo, IQCdir, IQCdif )

Each parameter is described in this chapter.

\subsubsection{Specifying Inputs}

SERI_QC1 requires data formatted for all three of the solar radiation elements (global horizontal, direct normal, and diffuse horizontal) even though one or two of the elements may be missing. Also required are the integration time (or interval) of the measurement and the ending time of the measurement in local standard time. If in units other than watts per square meter $\left(\mathrm{W} / \mathrm{m}^{2}\right)$, the solar irradiance data must be converted by your calling program to $\mathrm{W} / \mathrm{m}^{2}$ before the call to SERI_QC1. Table 9-1 gives conversions from several common measurement units to $\mathrm{W} / \mathrm{m}^{2}$. If a measurement value is missing, the input value for that field must be in excess of $8000 \mathrm{~W} / \mathrm{m}^{2}$ (e.g., 9900.0).

Table 9-1. Measurement Unit Conversion

\begin{tabular}{|l|c|}
\hline To convert from this unit to $\mathrm{W} / \mathrm{m}^{2}$ & $\begin{array}{l}\text { Multiply by this value } \\
(\mathrm{t}=\text { measurement integration time } \\
\text { in minutes })\end{array}$ \\
\hline \hline Joules per square meter $\left(\mathrm{J} / \mathrm{m}^{2}\right)$ & $0.01666 / \mathrm{t}$ \\
\hline Kilojoules per square meter $\left(\mathrm{kJ} / \mathrm{m}^{2}\right)$ & $16.66 / \mathrm{t}$ \\
\hline Megajoules per square meter $\left(\mathrm{MJ} / \mathrm{m}^{2}\right)$ & $16,666 / \mathrm{t}$ \\
\hline Kilowatt-hours per square meter $\left(\mathrm{kWh} / \mathrm{m}^{2}\right)$ & $60.0 / \mathrm{t}$ \\
\hline
\end{tabular}

The QC-zero file for the monitoring station must be present in the subdirectory where SERI_QC1 is being used. The QC-zero file contains geographical parameters of the site (latitude, longitude, and time zone), as well as boundary descriptors that define expected values for one- and two-component tests. SERI_QC1 also requires a site identifier to link the station with its QC-zero file.

The QC-zero file is created most easily using the QCFIT software included in the SERI QC package. See Chapter 7, Section 7.3 (page 87) for more information on the QC-zero file. The use of QCFIT is covered in Chapter 8.

Table 9-2 lists input variable descriptions. 
Table 9-2. Input Variable Descriptions

\begin{tabular}{|l|l|}
\hline \multicolumn{2}{|c|}{ CHARACTER* 20 } \\
\hline Site & Site identifier \\
\hline \multicolumn{2}{|c|}{ INTEGER } \\
\hline Iyear & The year, e.g., 1988 or simply 88 \\
\hline Month & The month of the year (1-12) \\
\hline Iday & The day of the month (1-31) \\
\hline Ihour & The hour of the day (0-24) \\
\hline Minute & The minute of the hour (0-59) \\
\hline Intrvl & The averaging interval in minutes (1-60) \\
\hline & \multicolumn{2}{|c|}{$R E A L$} \\
\hline Global & Global horizontal broadband solar radiation, $\mathrm{W} / \mathrm{m}^{2}$ \\
\hline Direct & Direct normal broadband solar radiation, $\mathrm{W} / \mathrm{m}^{2}$ \\
\hline Difuse & Diffuse horizontal broadband solar radiation, $\mathrm{W} / \mathrm{m}^{2}$ \\
\hline
\end{tabular}

A day runs from 00:01 to 24:00. The date and time fields represent the ending minute of the period during which the measurements were taken.

\subsubsection{Understanding Outputs}

SERI_QC1 returns an integer quality assessment flag for each of the three solar measurement components evaluated. These output variables are listed in Table 9-3.

Table 9-3. Output Variable Descriptions

\begin{tabular}{|l|l|}
\hline \multicolumn{2}{|c|}{ INTEGER } \\
\hline IQCglo & The quality assessment flag for the input variable "Global" \\
\hline IQCdir & The quality assessment flag for the input variable "Direct" \\
\hline IQCdif & The quality assessment flag for the input variable "Difuse" \\
\hline
\end{tabular}

The range of the quality assessment flags is $0-99$. By SERI_QC1 convention, these integer flags are referred to in this manual as two-digit numbers (00-99). These flags are explained in Chapter 6, Section 6.4 (page 46). 


\subsection{A Sample Calling Program}

SERI_QC1 is not a stand-alone program but rather a subroutine. Therefore, you must incorporate the subroutine in a calling program that presumably requires data evaluation as part of its processing. In this section, we provide general instructions to build a calling program using different scenarios of source solar irradiance data.

A fundamental concept of the subroutine is that SERI_QCl interfaces only with the calling program (and the QC-zero data files), not with any other data structure or file.

All irradiance data sent by the calling program to SERI_QC1 must have associated with it a site identifier, the date and time of the collection, and the interval of measurements as described in Section 9.1. This association need not be explicit within the data records from your file but must be explicitly sent with each call to SERI_QC1.

For example, a file of hourly data may be structured with a data record for each hour of the day, whether or not measurements were actually taken. Although each record would not be explicitly labeled with its associated hour, the file structure reveals the information. For example, the twelfth record corresponds to the twelfth hour. The calling program must in some way convert this implicit time representation into an actual hourly value. Another common data representation uses day-of-year rather than month and day. Again, it is required of the calling program that such data be converted to month and day values prior to the call to SERI_QC1: If the measurement end point is other than on-the-hour, a value must be assigned to the Minute variable (if unspecified, the default is zero). If your measurement time represents the beginning, midpoint, or anything other than the end point, the calling program must make a conversion to the end point time. In short, the calling program must read from a file or otherwise assign all the data required by SERI_QC1 before the subroutine is called.

The subroutine is called once for each record of solar data (a record being all solar components available for any given measurement interval); there is no provision for processing blocks or multiple records of data. In this context, if blocks of data must be processed (as would most likely be the case), that task is undertaken by the calling program. Thus, the SERI_QC1 subroutine may be called thousands of times when processing a file of data.

Although SERI_QC1 can work with a single solar component, it returns the most accurate assessment if all available components for a measurement interval are sent in the same call to the subroutine. In other words, if your data are segregated by component into different records or files, the calling program must invoke whatever algorithms are necessary to retrieve all components associated with a given measurement interval before SERI_QC1 is called. 
SERI_QC1 does not change any of the input variables. Once one of these variables has boen assigned by the calling program, it will not change from one call to the next. For example, once the site identifier has been set by the calling program, it need not be refreshed for each call to SERI_QC1.

The FORTRAN listing in Figure 9-1 is a sample calling program that reads a file of Albuquerque, NM, data in the default QCFIT format (described in Chapter 8, Section 8.2.1, page 100), passes each measurement record to the SERI_QC1 subroutine, then produces a simple report file indicating the number of times each flag was assigned. All source code and data files necessary to run this program are included in the SERI QC package.

\subsubsection{Comments on the Sample Program}

Note that the site identifier and interval are set only once at the beginning of the program. The values are retained throughout the rest of the program and are not refreshed for each call to SERI_QC1. The site identifier "ABQ" is used by SERI_QC1 to identify the QC-zero file associated with the site.

In the loop headed by statement 2000 , the program reads a record of data, then calls SERI_QC1. After the call, the flag values returned in IQCglo, IQCdir, and IQCdif are used to index and increment array variables for later reporting. Each array position corresponds to a flag; that position's value is incremented for each occurrence of the corresponding flag.

In the section headed by statement 5000 , the program opens an output file and loops through the arrays that were filled during the read/QC loop. For each index with a value greater than zero, a line is written to the report file. 
Program QA_ABQ

* Runs SERI_QC1 on data in QCFIT format and produces a list of flags.

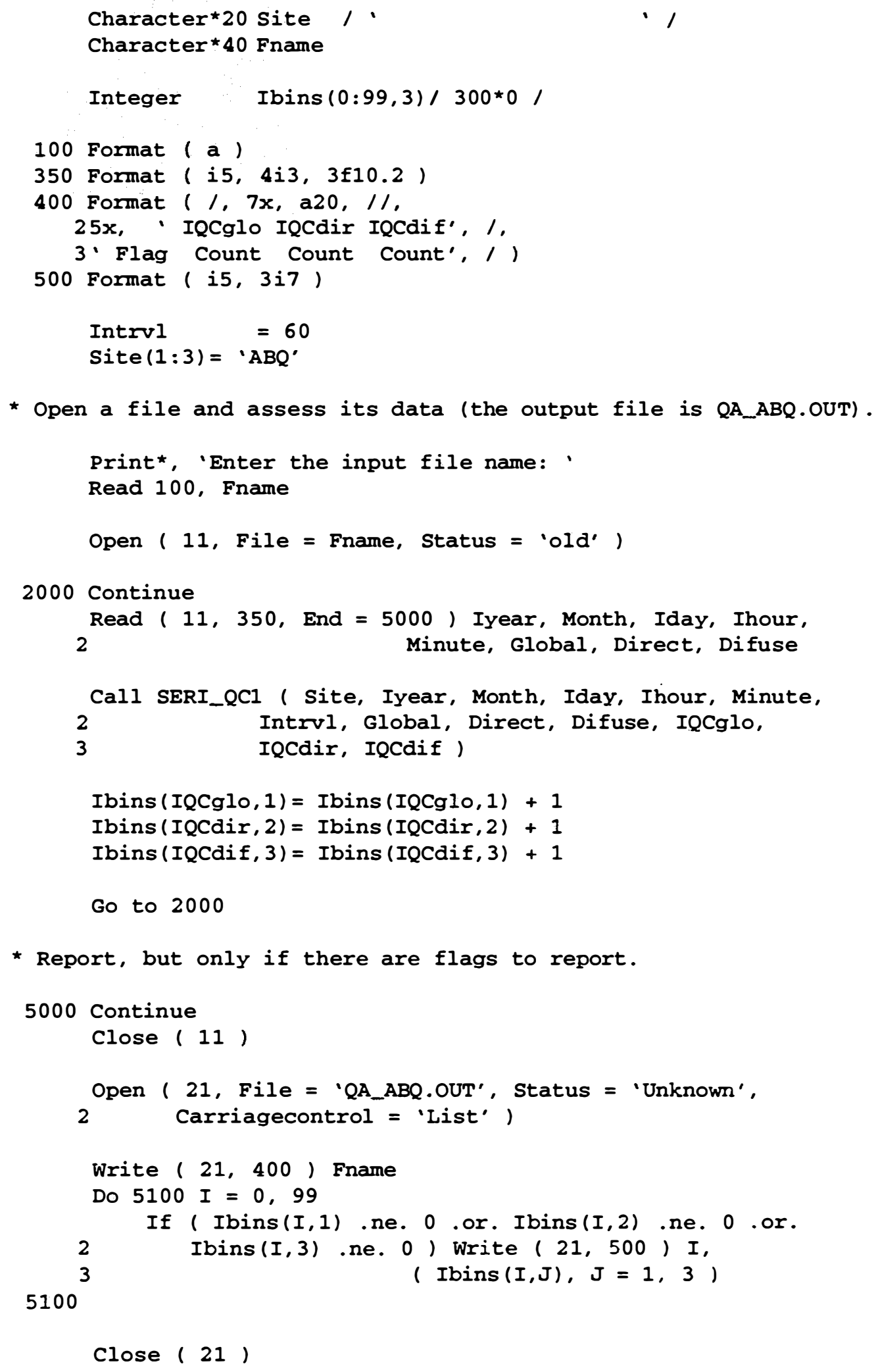


The report generated for Albuquerque, NM, for June data collected from 19881990 appears in Figure 9-2. This report was produced using a QC-zero file developed from 1977-1980 data.

\begin{tabular}{rrrr}
\multicolumn{4}{c}{ ABQ06C.QCF } \\
Flag & $\begin{array}{r}\text { IQCglo } \\
\text { Count }\end{array}$ & $\begin{array}{r}\text { IQCdir } \\
\text { Count }\end{array}$ Count \\
1 & 609 & 599 & 0 \\
2 & 577 & 577 & 0 \\
8 & 0 & 10 & 0 \\
12 & 2 & 1 & 0 \\
13 & 1 & 2 & 0 \\
16 & 1 & 1 & 0 \\
17 & 1 & 1 & 0 \\
24 & 2 & 0 & 0 \\
25 & 0 & 2 & 0 \\
32 & 6 & 0 & 0 \\
33 & 0 & 6 & 0 \\
99 & 0 & 0 & 1199
\end{tabular}

Figure 9-2. Report generated for Albuquerque, NM

For routine processing of solar radiation data, you would normally combine a flag and associated measurement in a new data file. Therefore, the calling program should write new data files with the flag value accompanying each solar irradiance value. We also recommend the generation of a report showing the results of the quality assessment, as illustrated in Figure 9-2.

\subsubsection{Writing Your Own Calling Program}

Initially, you may wish to modify the sample program to work with your data. First, you must create a QC-zero file for your site using the QCFIT program described in Chapter 8. If you opt to run QCFIT with your data in the QCFIT format, modifying this sample program is a simple matter of changing the site identifier, the interval (if not 60 minutes), and the file names.

If you wish to use another data format, the modification process becomes more difficult and must take into account the data requirements of SERI_QCl mentioned in this section. Remember, if your data contains only one or two components, the variable corresponding to the missing component must contain a value greater than 8000 (e.g., 9900.0). 
Once you have determined that the sample program is properly calling SERI_QC1 for your data, it can serve as a template for using SERI_QC1 in other programs. Maling sure that the site identifier and interval variables are assigned, the loop at statement 2000 in Figure 9-1 may be copied into another program. The QC flag values returned by each call to SERI_QC1 would be used according to the purpose of the program; for instance, statistical output, data filtering, or adding the flags to expanded data records for the evaluated components. 


\subsection{SERI_QC1 Subroutine Description}

A flow chart of SERI_QC1 is in Figure 9-3 at the end of this section. This diagram should help you understand the following sequence of events.

\subsubsection{Validating the Inputs}

Before examining the solar measurements, SERI_QC1 checks input data for validity. There are two sources of input data: the passed parameters (variables used to pass values to the subroutine) and the boundary information contained in the QC-zero file.

\section{Checking Passed Parameters}

SERI_QC1 issues the following error messages during checks on the input parameters:

Site: If this is not the same as the site specified during the last call (or this is the initial call), an attempt is made to open the corresponding QC-zero file. If the QC-zero file cannot be opened, the subroutine exits with this message:
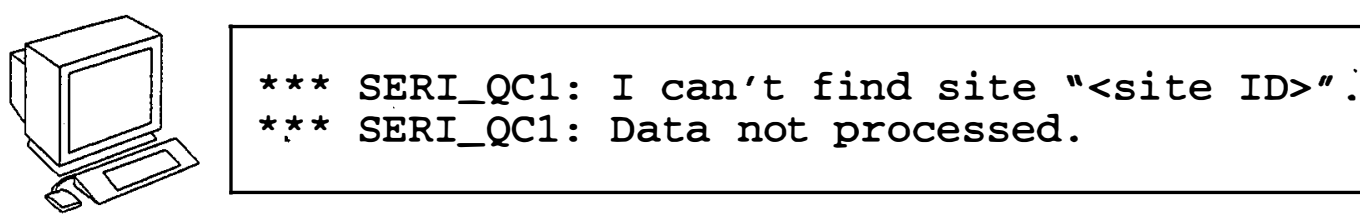

Iyear: No checks.

Month: If this is not an integer from 1 to 12 , the subroutine exits with this message:
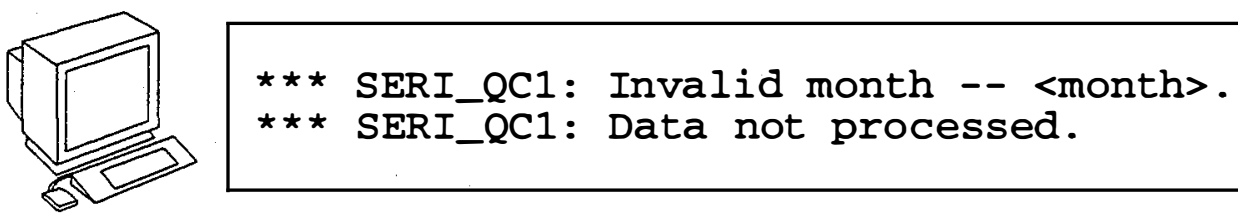

Iday: If this is not an integer from 1 to 31 , the subroutine exits with this message:

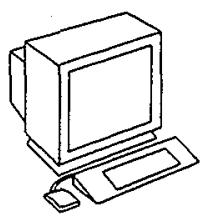

$$
\begin{array}{ll}
\star \star \star & \text { SERI_QC1: Invalid day }--<\text { Iday }>\text {. } \\
\star \star \star & \text { SERI_QC1: Data not processed. }
\end{array}
$$


Ihour: If this is not an integer from 0 to 24 , the subroutine exits with this message:
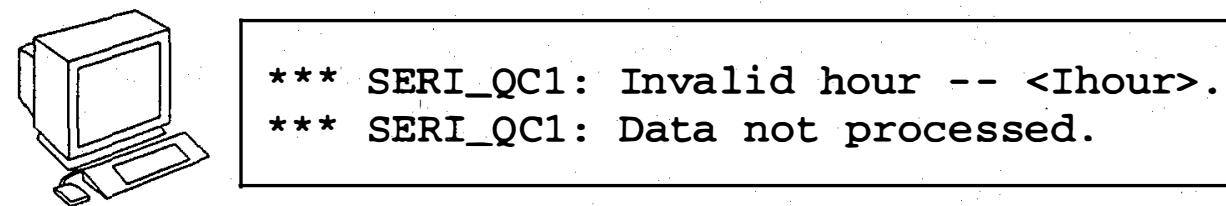

Minute: If this is not an integer between 0 and 59, the subroutine exits with this message:
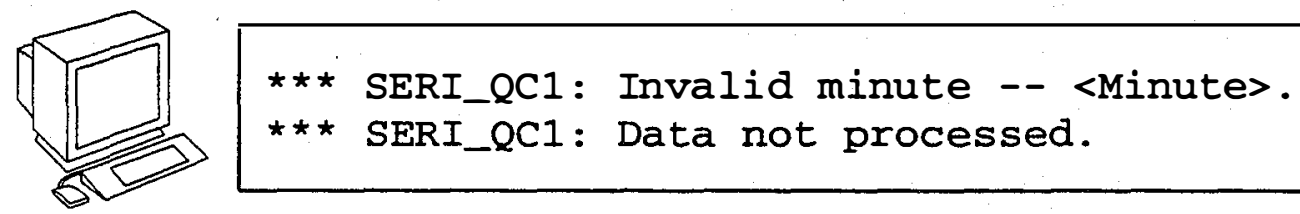

A check is made on Minute and Ihour for a valid time combination. If the time is not within 00:01 to 24:00, the subroutine exits with this message:
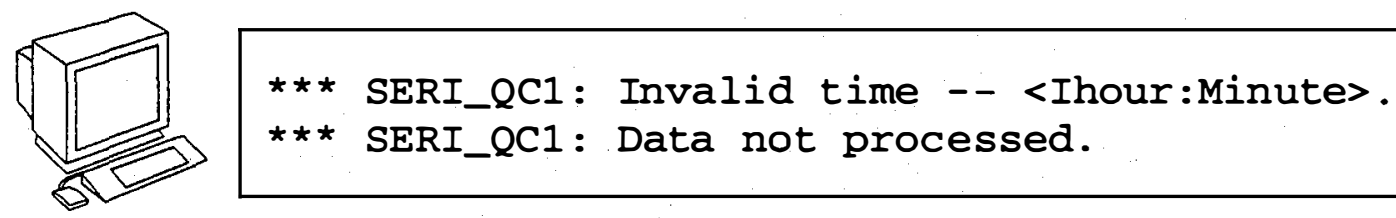

Intrvl: Not checked.

Global, Direct, Difuse: Checked in the context of SERI_QC1.

\section{Checking the QC-zero File}

SERI_QC1 performs the following checks on the QC-zero file:

- Unexpected EOF (End of File): End of file is reached when more data were expected.

- Positioning colon missing or out of position: The colons in the latitude, longitude, and time zone lines are used as a reference point to assure that the lines are as expected.

- Month label missing or out of place: The three-character month abbreviation for the desired month is compared with its expected position in the file.

- A read error on any numeric field. 
Any of these problems cause the subroutine to exit with this message:

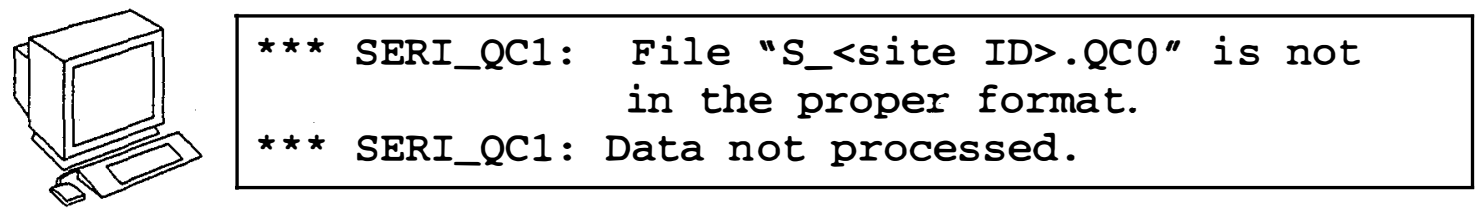

If any required field read from the boundary record is undefined (zero), the subroutine exits and this message appears:

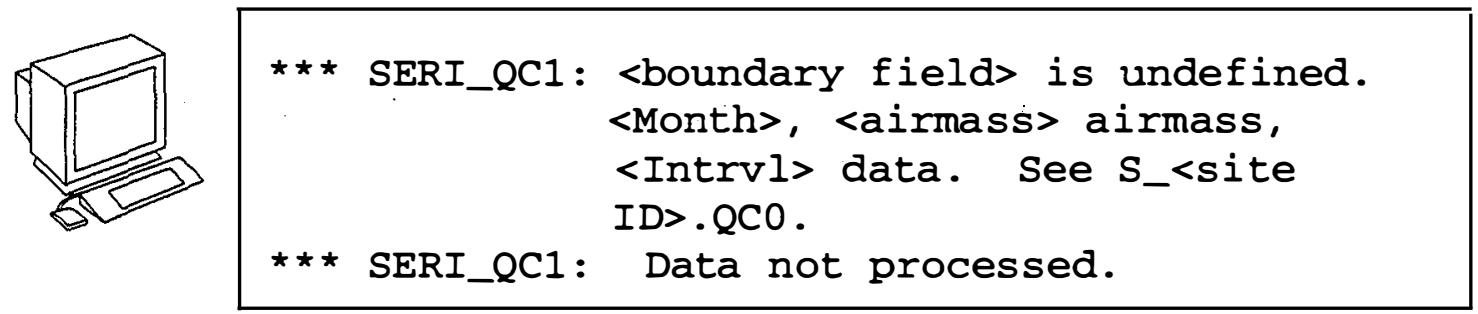

All flags (IQCglo, IQCdif, IQCdir) are set to 00 for any of the above conditions.

\subsubsection{Trivial Category Tests}

Data that do not meet the requirements for normal one-, two-, or three-component tests are put into a "trivial" category. The following tests are performed.

Missing Data - If the value of any of the solar input parameters exceeds 8000 , the parameter is considered missing, and its corresponding QC flag (IQCglo, IQCdir, IQCdif) is set to 99. The parameter is not used in subsequent tests.

Nighttime Values - If the position of the sun is below the horizon throughout the time interval, the nighttime check is performed. Any solar input parameter with a value $<-10 \mathrm{~W} / \mathrm{m}^{2}$ is given a flag of 07 ; if its value is $>10 \mathrm{~W} / \mathrm{m}^{2}$, it receives a flag of 08 . Otherwise, its flag is set to 01 . No subsequent tests are performed.

Twilight Values - Although not necessarily trivial for some locations and applications, data with solar zenith angles between $80^{\circ}$ and $90^{\circ}$ at the midpoint of the sunup measurement period are subjected to only a modified one-component test (see Chapter 6, Section 6.4.1, page 46 for an explanation).

The minimum limit for all components is set to $-10 \mathrm{~W} / \mathrm{m}^{2}$. Any component with a measurement $<-10 \mathrm{~W} / \mathrm{m}^{2}$ is given a flag of 07 . A flag of 08 is assigned when a $\mathrm{K}$-value exceeds the single-component high air mass maximum. However, when ETR $<25 \mathrm{~W} / \mathrm{m}^{2}$, IQCglo is set to 08 if global $>10 \mathrm{~W} / \mathrm{m}^{2}$ and $\mathrm{Kt}>\mathrm{Kt}_{\max }+0.10$. Otherwise, the flag is set to 01 . No subsequent tests are performed. 


\subsubsection{One-Component Tests}

One-component tests (described in Chapter 6, Section 6.4.1, page 46) are performed on all irradiance components that do not fall into the trivial categories just discussed. The resultant flags are used by subsequent tests.

- IQCglo is set to 07 if $\mathrm{Kt}$ is $<0.05$, and is set to 08 if $\mathrm{Kt}$ is $>\mathrm{Kt}_{\max }+0.10$; otherwise, IQCglo is set to 01.

- IQCdir is set to 07 if Direct is $<-10 \mathrm{~W} / \mathrm{m}^{2}$ and is set to 08 if $\mathrm{Kn}$ is $>\mathrm{Kn}_{\max }$; otherwise, IQCdir is set to 01 .

- IQCdif is set to 07 if $\mathrm{Kd}$ is $<0.03$, and is set to 08 if $\mathrm{Kd}>\mathrm{Kd}_{\max }$; otherwise, IQCdif is set to 01 .

Any parameter with a flag other than 01 is not used in subsequent tests.

\subsubsection{High-Direct Test}

If $\mathrm{Kt}$ and $\mathrm{Kn}$ pass the one-component test (flag of 01 ), the following flags are assigned if $\mathrm{Kn}>\mathrm{Kt}$ :

$$
\begin{array}{ll}
\text { Kt/Kn relation } & \text { Flag assignment } \\
\mathrm{Kn}>\mathrm{Kt}+0.20 & \text { IQCglo }=\mathrm{IQCdir}=97 \\
\mathrm{Kt}+0.20>\mathrm{Kn} \geq \mathrm{Kt}+0.15 & \text { IQCglo }=\mathrm{IQCdir}=96 \\
\mathrm{Kt}+0.15>\mathrm{Kn} \geq \mathrm{Kt}+0.10 & \text { IQCglo }=\mathrm{IQCdir}=95 \\
\mathrm{Kt}+0.10>\mathrm{Kn} \geq \mathrm{Kt}+0.05 & \text { IQCglo }=\mathrm{IQCdir}=94
\end{array}
$$

If one of the above conditions is met, no further tests are performed.

\subsubsection{Three-Component Tests}

The three-component tests and resulting flags are described in Chapter 6, Section 6.4.3 (page 49). These tests are performed when all three parameters passed the previous tests (one-component and high-direct) with a flag of 01 . If the threecomponent test detects a discrepancy of $<0.03$, but the subsequent two-component test detected a discrepancy of $>0.05$, all three flags are set to 09 . Otherwise, the flags generated by the three-component test are retained.

\subsubsection{Two-Component Tests}

The two-component tests and resulting flags are described in Chapter 6, Section 6.4.2 (page 47). These tests are performed when at least two parameters passed the one-component and high-direct tests with flags of 01 . 
The irradiance components with flags meeting these criteria are subjected to Gompertz testing. Because Gompertz testing uses $\mathrm{Kn}$ and $\mathrm{Kt}$, missing $\mathrm{Kn}$ or $\mathrm{Kt}$ values are calculated from the two available components using the following equations:

$$
\begin{aligned}
& K t=K n+K d \\
& K n=K t-K d
\end{aligned}
$$

If all three components qualify for Gompertz testing, the three-component test is performed first. If the three-component test sets the flags to values other than 03 , Gompertz testing is not applied. Otherwise, the Gompertz test is performed on Kt and $\mathrm{Kn}$ to determine if the flags should have been values of 03 or 09 . 


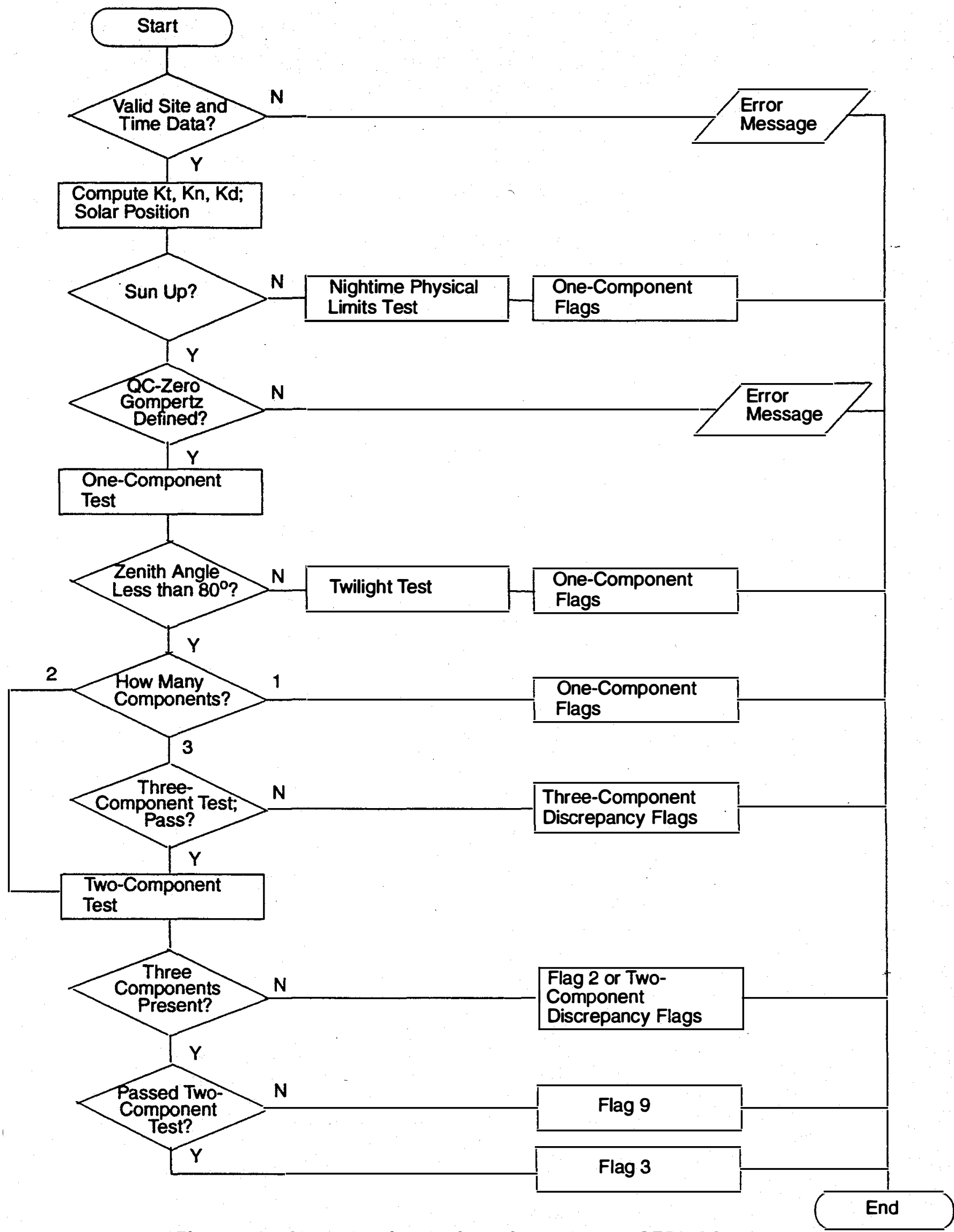

Figure 9-3. Chart showing the flow of data through SERI_QC1 


\subsection{SERI_QC1 Program Notes}

SERI_QC1 does not actively use the Gompertz function for calculating the expected boundaries. Instead, the initiated "curve" array stores precalculated Kt Gompertz values as a function of $\mathrm{Kn}$ for the leftmost (first) position of each shape. The other 19 positions for each shape are simple linear offsets in multiples of $0.025 \mathrm{Kt}$ from the curve defined in the array. By using precalculated values rather than the function, the computational operations involved are reduced from a timeconsuming double exponential to the quicker operations of addition, multiplication, and a memory fetch; thus, this approach offers greater speed at a minimal cost for storage.

All flags are initially set to zero in the event SERI_QC1 fails as a result of inadequate or invalid input information. 



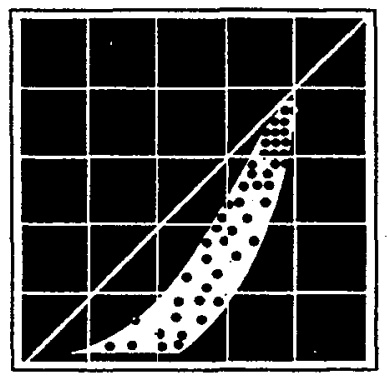

\section{Appendix \\ QCFIT Plots for 12 NOAA Stations}

This appendix contains QCFIT plots for the months of January, April, July, and October for the 12 National Oceanic and Atmospheric Administration (NOAA) stations listed in Table A-1. These stations represent a wide range of climate conditions found in the United States, encompass latitudes from $\mathrm{N} 26^{\circ}$ to $\mathrm{N} 47^{\circ}$, and include locations on both coasts. Hence, most of the conditions likely to be found in the conterminous United States are represented herein. They can be used as a reference to compare with plots for climatically similar locations.

These are not hypothetical plots representing perfect data sets. Rather, these plots were generated during the quality assessment of data collected at NOAA-National Weather Service stations from 1977 through 1980. All of the data collected by each station during those years were included; therefore, good and bad data are shown. It is likely that some boundaries would be changed if more good quality data were to become available for some of these locations. Furthermore, a different user of QCFIT might assign different boundaries to some of the data. Brief comments regarding the plots for each station are offered for instructional purposes. 
Table A-1. NOAA Stations with QCFIT Plots

\begin{tabular}{|l|c|c|c|}
\hline \multicolumn{1}{|c|}{ Station } & Code & WBAN & Page \\
\cline { 1 - 4 } Albuquerque, NM & ABQ & 23050 & 168 \\
\hline Bismarck, ND & BIS & 24011 & 170 \\
\hline Brownsville, TX & BRN & 12919 & 172 \\
\hline Caribou, ME & CAR & 14607 & 174 \\
\hline Columbia, MD & COL & 03945 & 176 \\
\hline Lake Charles, LA & LAK & 03937 & 178 \\
\hline Los Angeles, CA & LAX & 23174 & 180 \\
\hline Medford, OR & MED & 24225 & 182 \\
\hline Montgomery, AL & MRY & 13895 & 184 \\
\hline Phoenix, AZ & PHX & 23183 & 186 \\
\hline Salt Lake City, UT & SLC & 24127 & 188 \\
\hline Sterling, VA & STR & 93734 & 190 \\
\cline { 1 - 2 } & & &
\end{tabular}




\section{Albuquerque, NM}

Albuquerque is representative of arid to semi-arid locations in the Southwest. With the exception of the winter season, clear skies dominate the weather, producing dense clusters of data with high $\mathrm{Kt}$ and $\mathrm{Kn}$ values. Considering only these plots, the $\mathrm{Kt}_{\max }$ limits for both July and October could be reduced. However, these values were selected to achieve uniformity with the limits set for adjacent months. If data for other years were available, it is quite possible that data with $\mathrm{Kt}$ values consistent with these limits would be observed for July and October as well as the adjacent months. In general, these data exhibit the characteristics of good quality data from a well maintained station.

\section{Bismarck, ND}

Bismarck is at a northern location ( $\left.46^{\circ}-46^{\prime}\right)$ that receives about 40 in. $(100 \mathrm{~cm})$ of snow each winter. This accounts for the steep right-hand boundaries for January and April, resulting from high $\mathrm{Kt}$ values even with low $\mathrm{Kn}$ values. These effects are produced by multiple scattering of solar radiation between a snow covered surface and clouds. In January at this latitude, there were only a few hours for which the solar elevation was high enough to place data in the medium air mass range. Therefore, the boundaries for these data were based on boundaries for the two adjacent months. In many instances, QCFIT results for adjacent months and/or adjacent stations have been used to compensate for lack of data or data of questionable quality. The data for Bismarck are also indicative of a well maintained station.

\section{Brownsville, TX}

Brownsville is one of the lowest latitude cities ( $\left.\mathrm{N}^{2} 5^{\circ}-54^{\prime}\right)$ in the United States (same latitude as Miami). Its weather is dominated by cloudy skies except for the summer months. This results in a more uniform distribution of data from high to low $\mathrm{Kn}$ and $\mathrm{Kt}$ values. Significantly lower $\mathrm{Kt}_{\max }$ and $\mathrm{Kn}_{\max }$ limits are noted for this station as compared to Albuquerque. The data for July exhibit problems that might be attributed to a tilted sensor, but the medium and high air mass ranges are inconsistent in this regard. The data indicate that a problem existed, but we cannot identify the source of the problem. If we were to examine this data by yearly subsets, we might find the problem data isolated to one year. 


\section{Caribou, ME}

Caribou is the highest latitude ( $\mathrm{N}^{\circ}-52^{\prime}$ ) station in the conterminous United States. This region receives an average of $113 \mathrm{in}$. $(287 \mathrm{~cm})$ of snow each winter. Hence, the effects of multiple reflections between the surface and clouds is even more noticeable than at Bismarck. Notice that some $\mathrm{Kt}$ values at low $\mathrm{Kn}$ levels exceed $\mathrm{Kt}$ values under clear skies. It is likely that the medium air mass, right-hand boundary for January should be one or two steps farther to the right. There was very little data for the adjacent months (none in December) on which to base its position. The wide distribution of the data during all months is likely the result of the high latitude (low solar elevations) and the varying surface albedo and cloud conditions; there is no reason to doubt the quality of the data, although errors may have contributed to the scatter.

\section{Columbia, MO}

On the average, Columbia receives 5 or 6 in. (13 or $15 \mathrm{~cm}$ ) of snow during each month from December through March. This is evidenced by the wide boundaries of the data for January, even though the quantity of data collected for this month was quite small. Cloudy skies are common except during the months of October and November; the greater concentration of data at high $\mathrm{Kn}$ and $\mathrm{Kt}$ values is evident in October. With the exception of October, the small sample sizes makes the boundary selections uncertain. They should be reevaluated when more data are available. However, the data that are available appear to be of good quality.

\section{Lake Charles, LA}

Lake Charles is a humid, subtropical site close to the Gulf of Mexico. The low $\mathrm{Kt}_{\max }$ and $\mathrm{Kn}_{\max }$ limits during January are the result of a mean cloud cover of 7.1 tenths. The high cloud cover during all months results in a generally uniform distribution of data within the boundaries. The data sets were rather small for several of the air mass ranges for certain months. Therefore, these boundaries are subject to change.

\section{Los Angeles, CA}

The NWS station is located on the coast at the Los Angeles International Airport. Air pollution is moderated most of the time by the westerly ocean breeze. Radiational cooling results in the formation of fog or low stratus clouds at night, particularly during the spring and summer months. These clouds dissipate during the morning, resulting in the dominance of clear skies during midday low air mass hours. This results in a steep right-hand boundary, since the scatter of the data is primarily the result of varying aerosols and water vapor. A bi-modal distribution for high air mass conditions (cloudy during early morning hours and clear in the afternoon) is particularly noticeable during April. 


\section{Medford, OR}

Medford is located in a valley surrounded by mountains on all sides. Late fall, winter, and early spring are damp and cloudy, under the influence of marine air. The rest of the year is sunny, dry, and warm due to prevailing continental winds during these months. The effect of these seasonal patterns are apparent; note especially the changes between April (data uniformly distributed over the Kn range) and July (data concentrated at high $\mathrm{Kn}$ values). The high air mass data in October indicate the possibility of a tilted sensor. The rather wide distribution of the data could be the result of fog and smoke, which sometimes collect in the valley.

\section{Montgomery, AL}

Montgomery is cloudy most of the year, with the lowest average cover occuring in October (4.5 tenths). During the winter there are frequent shifts from mild, moist air from the Gulf of Mexico to dry, cool continental air. This may be the cause of the bi-modal character of the scatter plot for January. One of the nation's largest seasonal changes in aerosol optical depth is observed at Montgomery (high in the summer and low in the winter). This results in relatively low $\mathrm{Kn}_{\max }$ limits during July as compared to January. Both $\mathrm{Kn}_{\max }$ and $\mathrm{Kt}_{\max }$ limits should probably be placed lower for the month of July.

\section{Phoenix, AZ}

Phoenix has an arid, very sunny climate with percent of possible sunshine varying from $77 \%$ in the winter to $95 \%$ in the summer. In July, cloud cover usually builds up during late afternoon hours and then dissipates during the morning. This produces the narrow cluster of data at high $\mathrm{Kn}$ values for the low air mass range and increasing scatter of the data for medium and high air mass ranges. The lower elevation and more turbid skies at Phoenix result in lower $\mathrm{Kn}_{\max }$ and $\mathrm{Kt}_{\max }$ limits than are found at Albuquerque, NM.

\section{Salt Lake City, UT}

Salt Lake City is quite cloudy during the winter and spring months (more than 7 tenths cloud cover in December and January) and relatively clear during the summer ( 3.5 tenths) and fall months. Just under 60 in. $(150 \mathrm{~cm})$ of snow falls during an average year, the effects of which can be seen in the scatter plots for January. The $\mathrm{Kt}_{\max }$ limits for both April and July were set too high for this station. Also, the left-hand boundary for the medium air mass range in July appears to be too far to the left. The scattering of data above the diagonal for January, high air mass indicates a problem with some of the data. 


\section{Sterling, VA}

This site is located close to the Dulles International Airport, serving the

Washington, D.C., area. There is little seasonal variation in cloud cover, ranging from 5.3 tenths in October to 6.7 tenths in January and March. This is apparent in the rather uniform distribution of data across all $\mathrm{Kn}$ values during all months of the year. Although there is significant snowfall during the winter (an average of 6 in. $[15 \mathrm{~cm}]$ in both January and February), the forested landscape and short duration of snow cover produce little evidence of ground-to-cloud scattering of solar radiation. 


\section{QCFIT Plots}



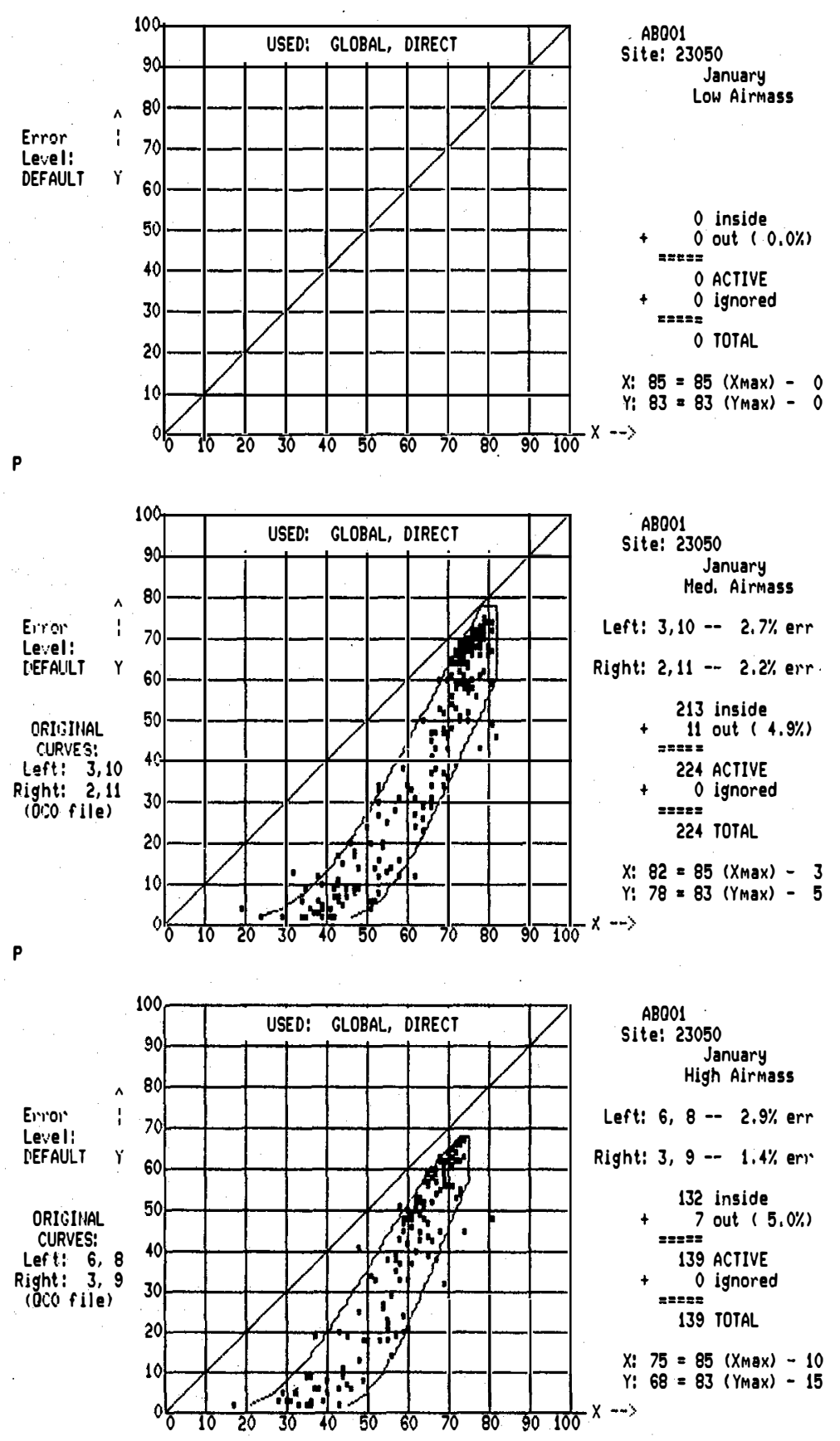
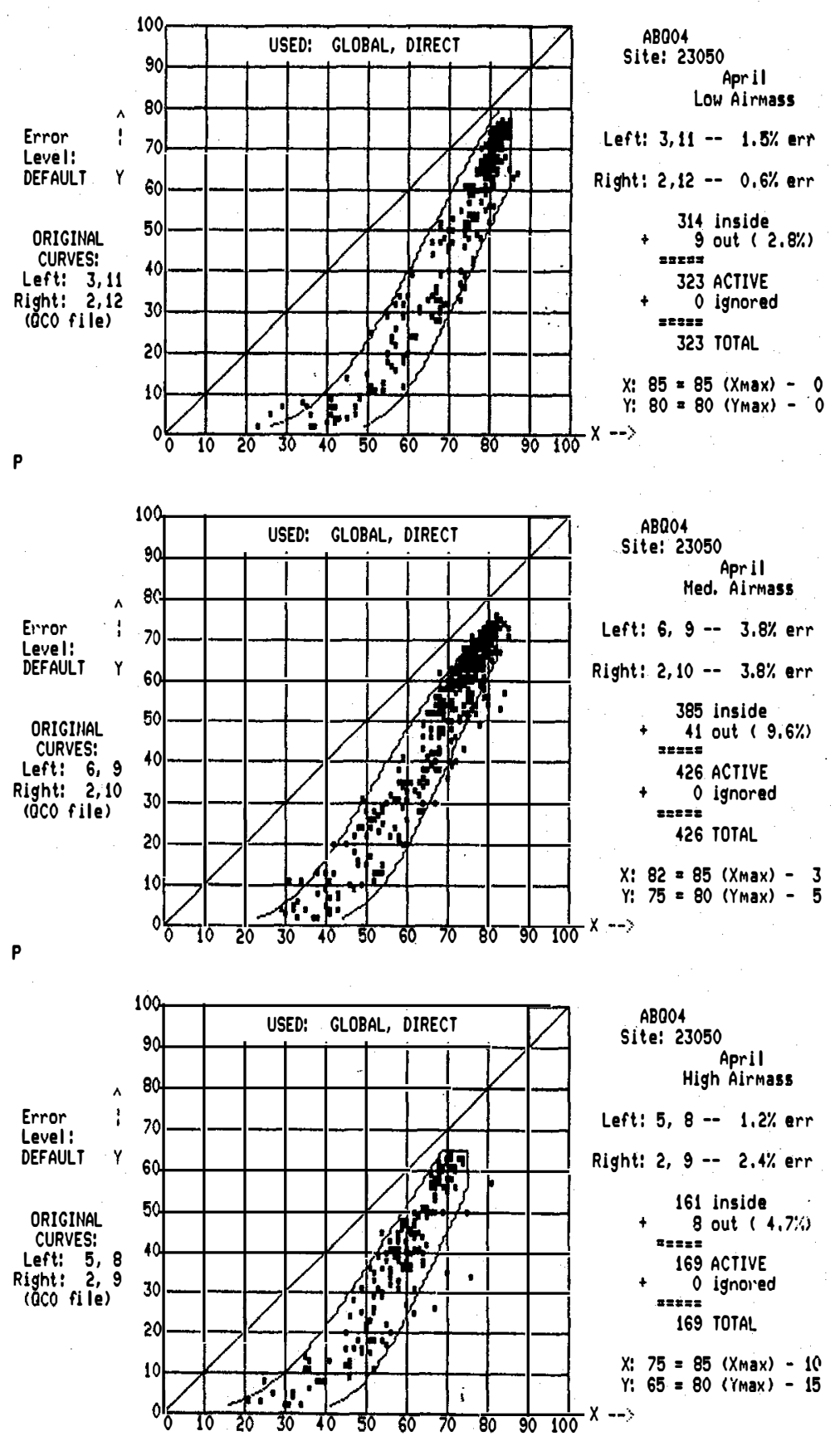

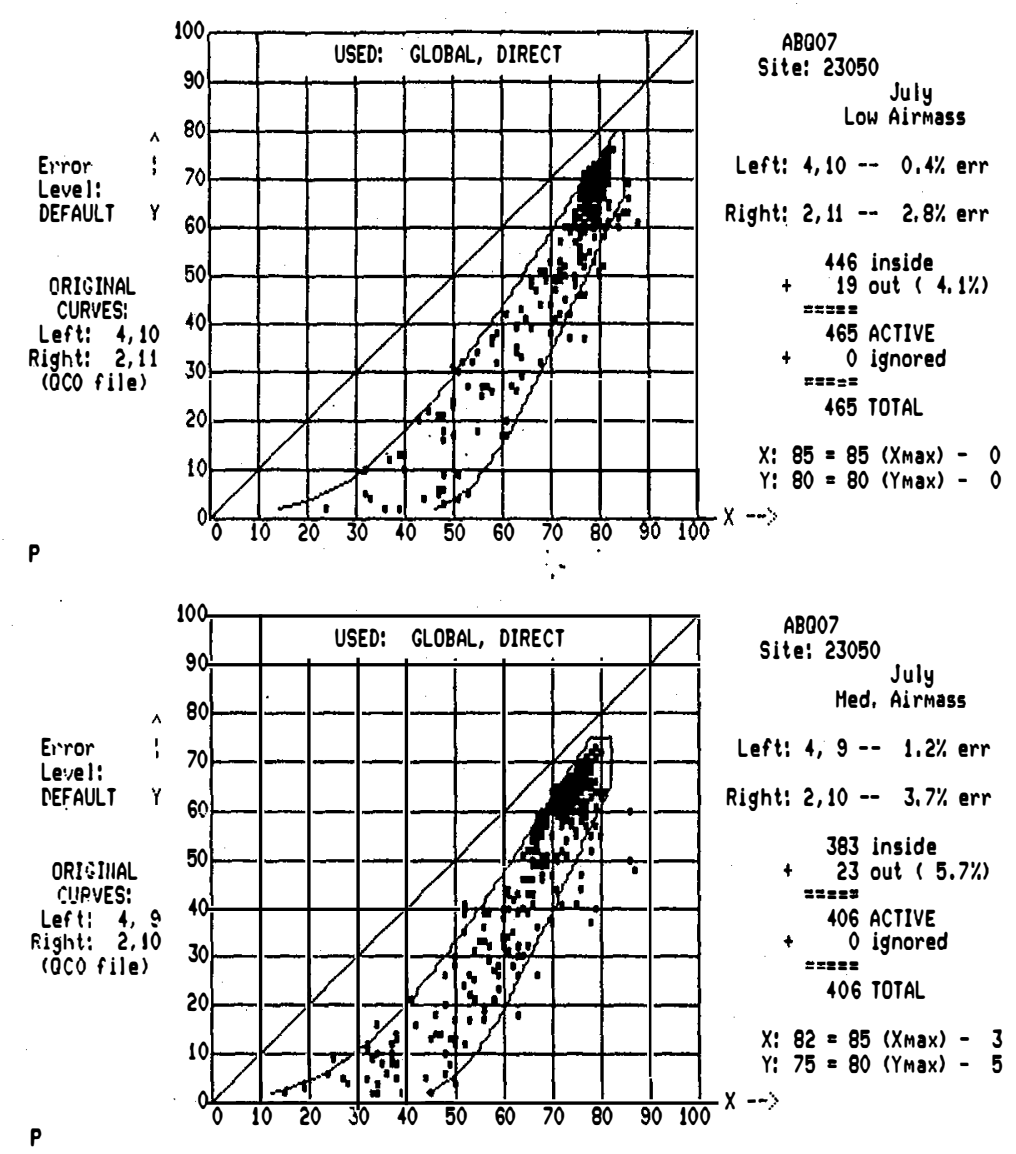

P
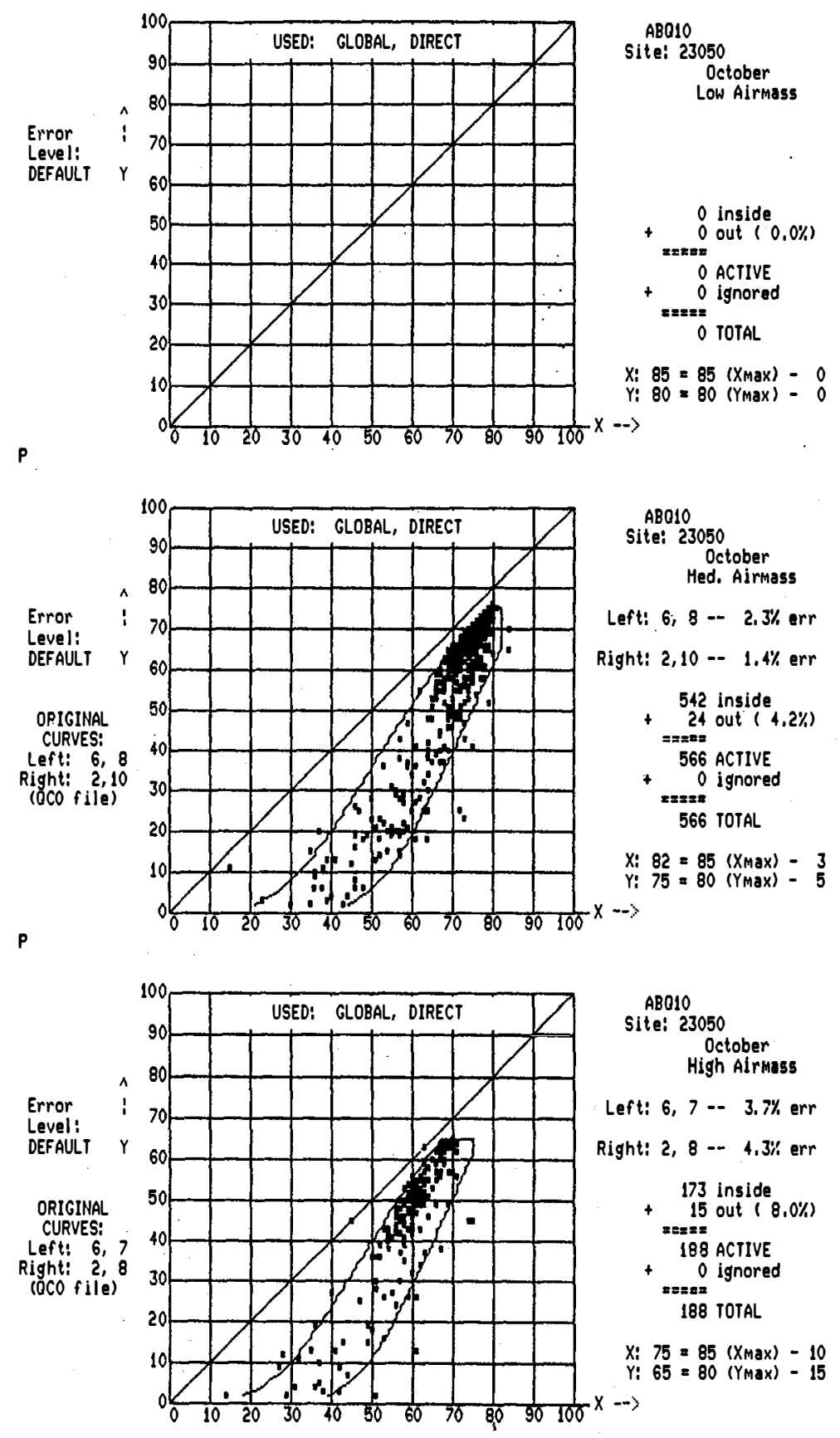


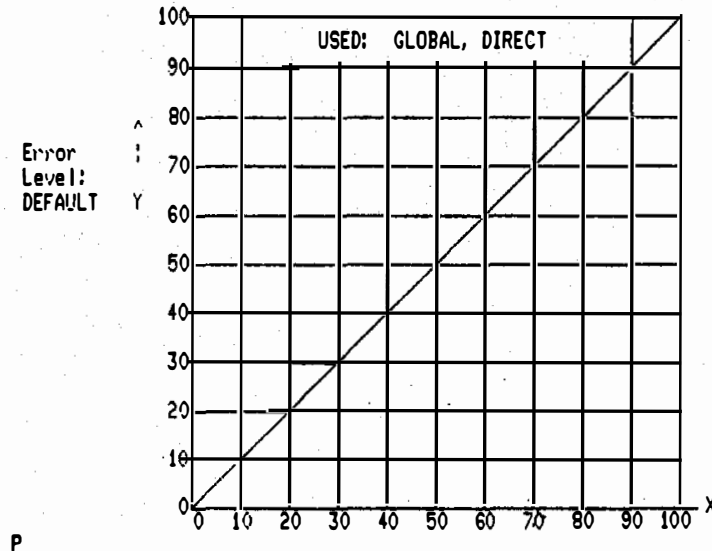

bis01
Site! 24011

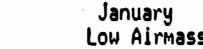

0 inside
$+\quad 0$ out $(0.0 \%)$

$== \pm=\Rightarrow$ ACTIVE

$+\quad 0$ ignored

0 TOTAL

$X: 88=88\left(X_{\text {max }}\right)-0$ $Y: 80=80\left(Y_{\text {max }}\right)-0$
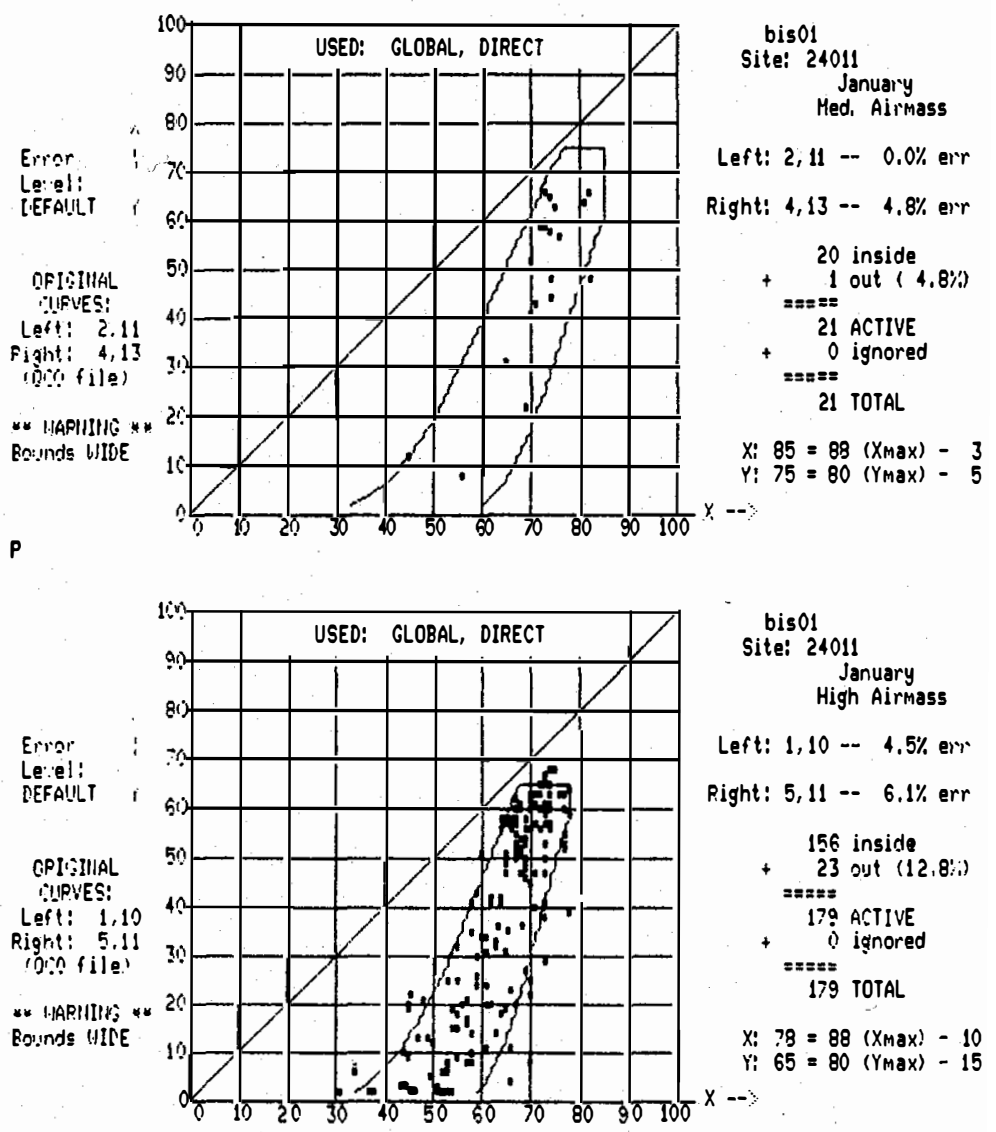
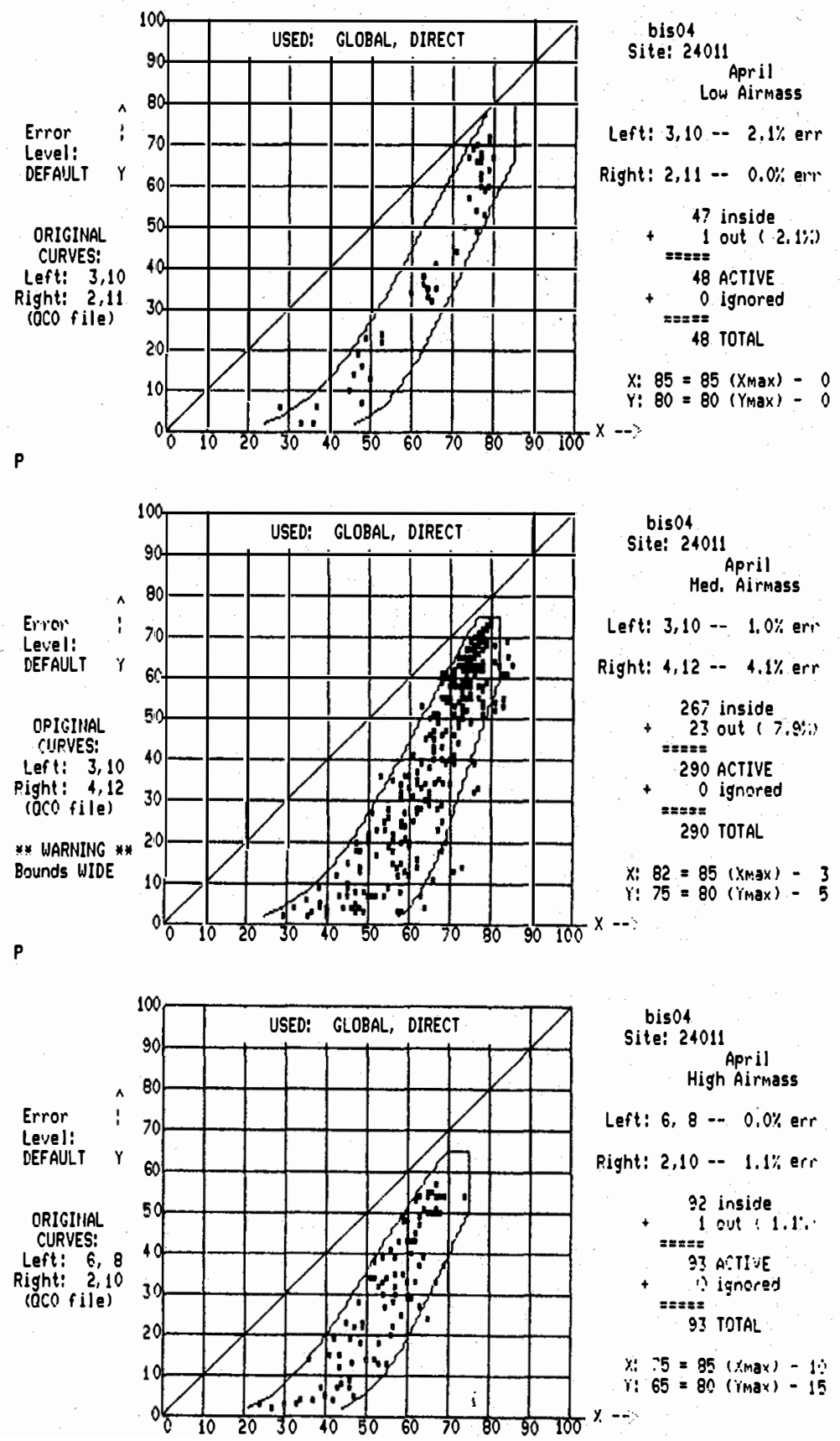


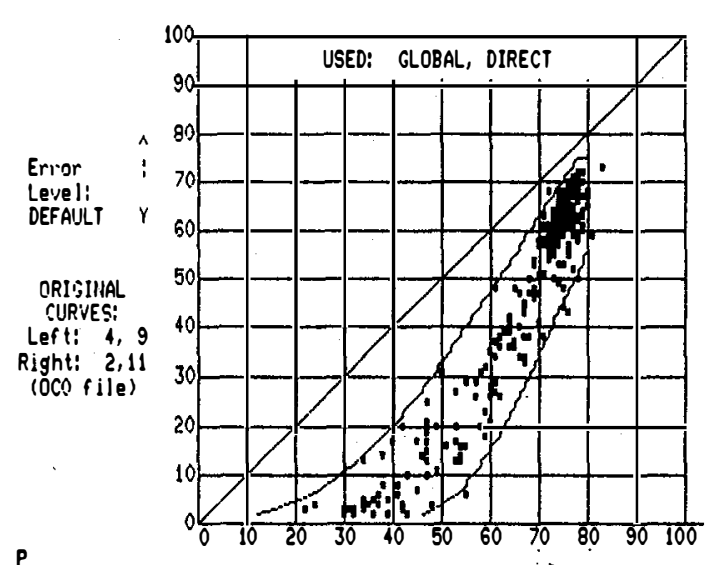

bis07

$$
\begin{gathered}
\text { July } \\
\text { Low Airmass }
\end{gathered}
$$

Left: $4,9-0.3 \% \mathrm{err}$ Right: 2,11 -- $1.1 \% \mathrm{err}$

345 inside
$+\quad 7$ out
$+2.0 \%)$

$==352$ ACTIVE

$\begin{array}{r}352 \text { ACrive } \\ +\quad 0 \text { ignored } \\ \hline\end{array}$

352 TOTAL

$X: 80=80\left(X_{\text {max }}\right)-0$
$Y: 75=75\left(Y_{\text {max }}\right)-0$ $x \rightarrow$

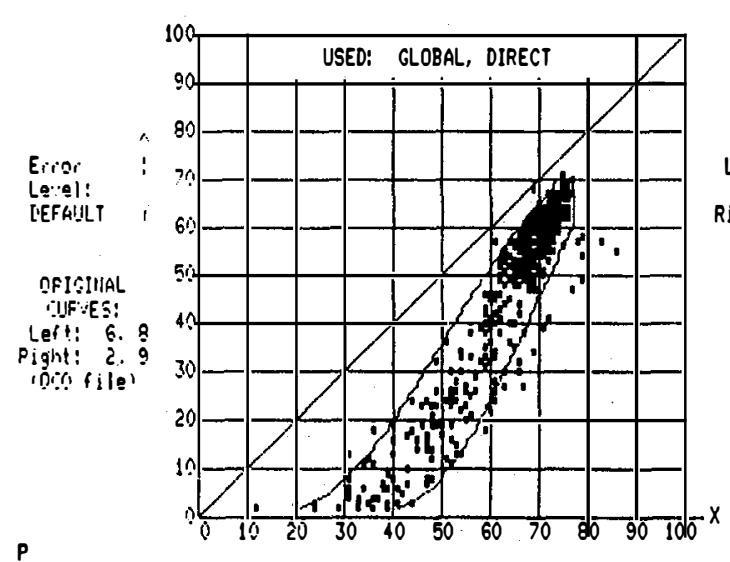

bis07
Site: 24011

$$
\text { Med. Airmass }
$$

Left: $6,8-2 \quad 1,5 \%$ err Right: 2, 9 -- 2.8\% err

\begin{tabular}{l}
432 inside \\
$+\quad 27$ out ( $5.9 \%)$ \\
\hline
\end{tabular}

459 ACTIVE

459 ACrive
$+\quad 0$ ignored

459 TOTAL

$X: 77=80\left(X_{\text {max }}\right)=3$
$Y: 70=75\left(Y_{\max }\right)=5$

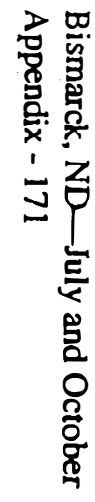

p
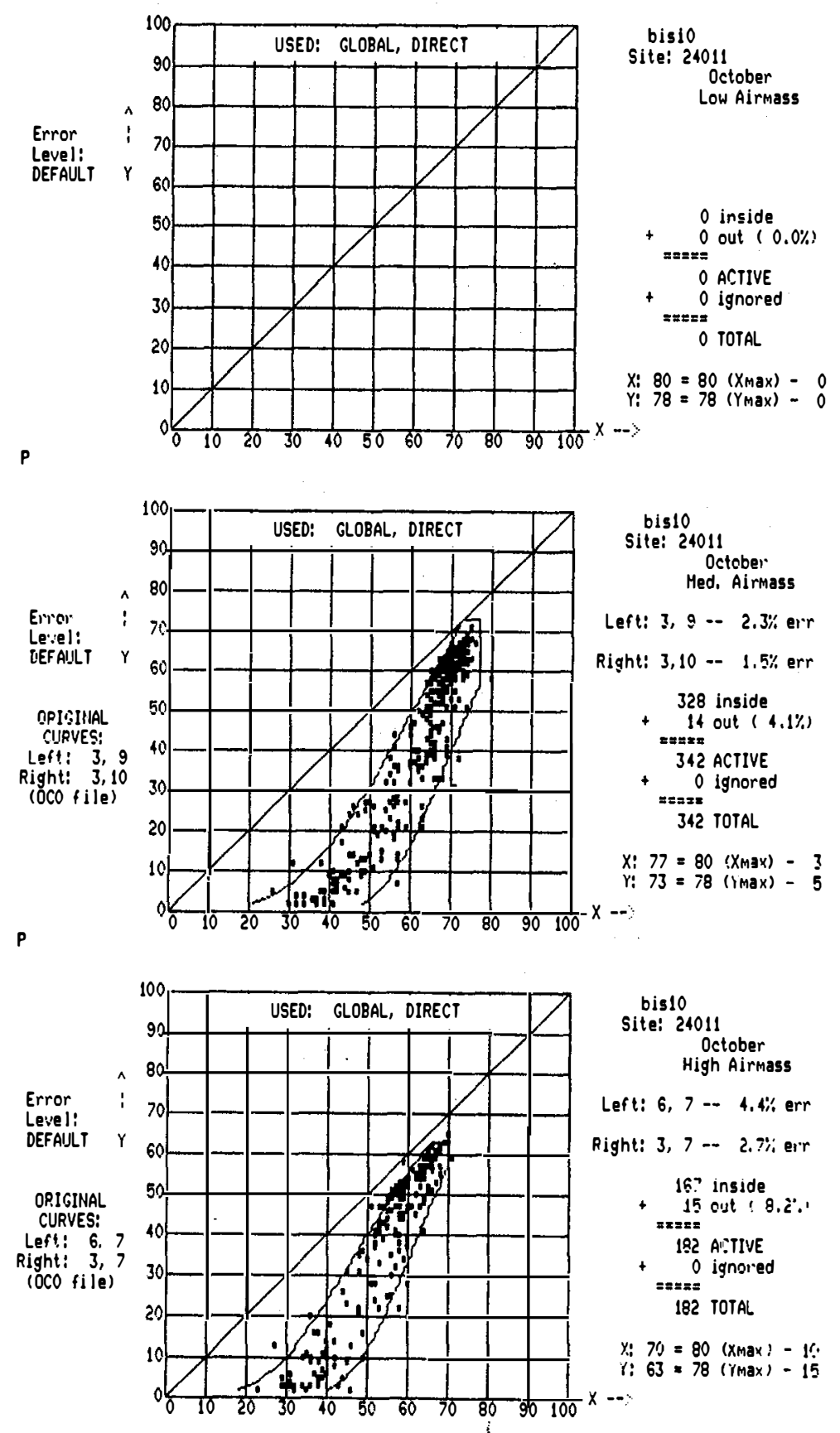


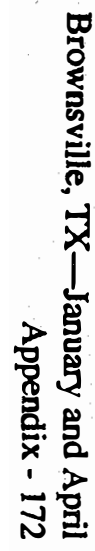

brnol

Site: 12919 Low Airmass

$$
\begin{gathered}
0 \text { inside } \\
0 \text { out }(0.0 \%) \\
=z=z \text { ACTIVE } \\
0 \text { ACIVE } \\
0 \text { ignored } \\
==== \\
0 \text { TOTAL }
\end{gathered}
$$

$X: 80=80\left(X_{\max }\right)-0$
$Y: 75=75\left(Y_{\max }\right)-0$ $-\rightarrow$
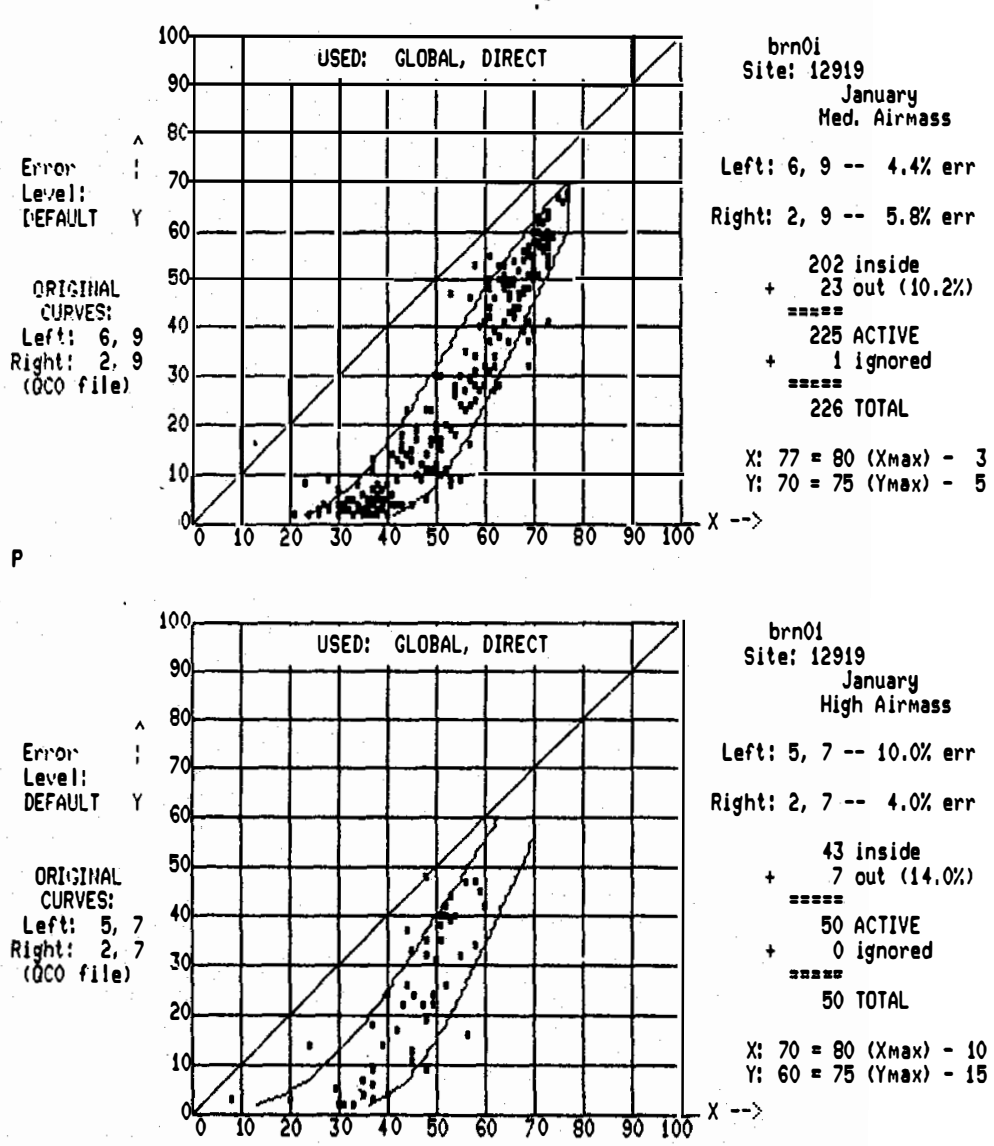
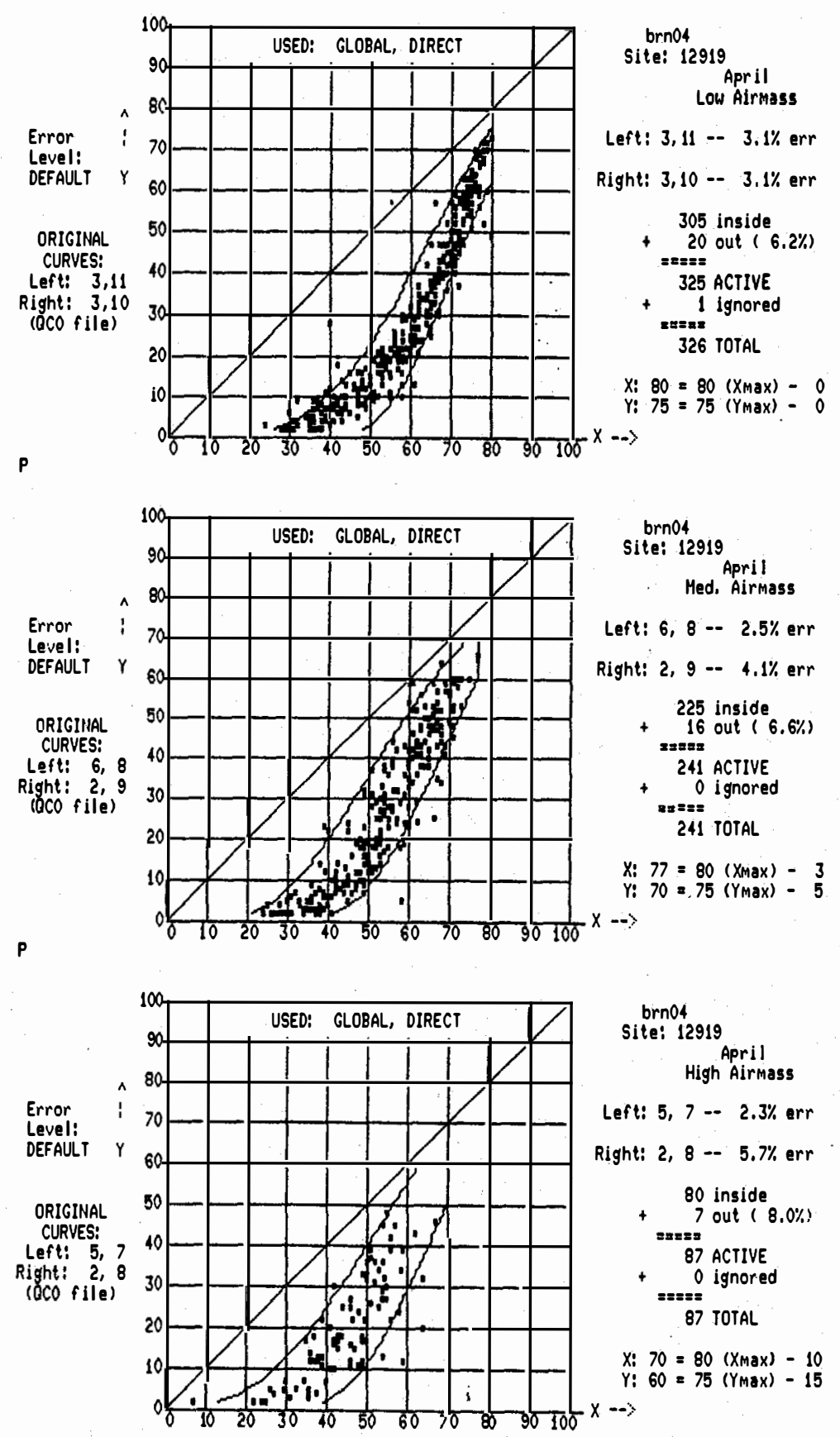

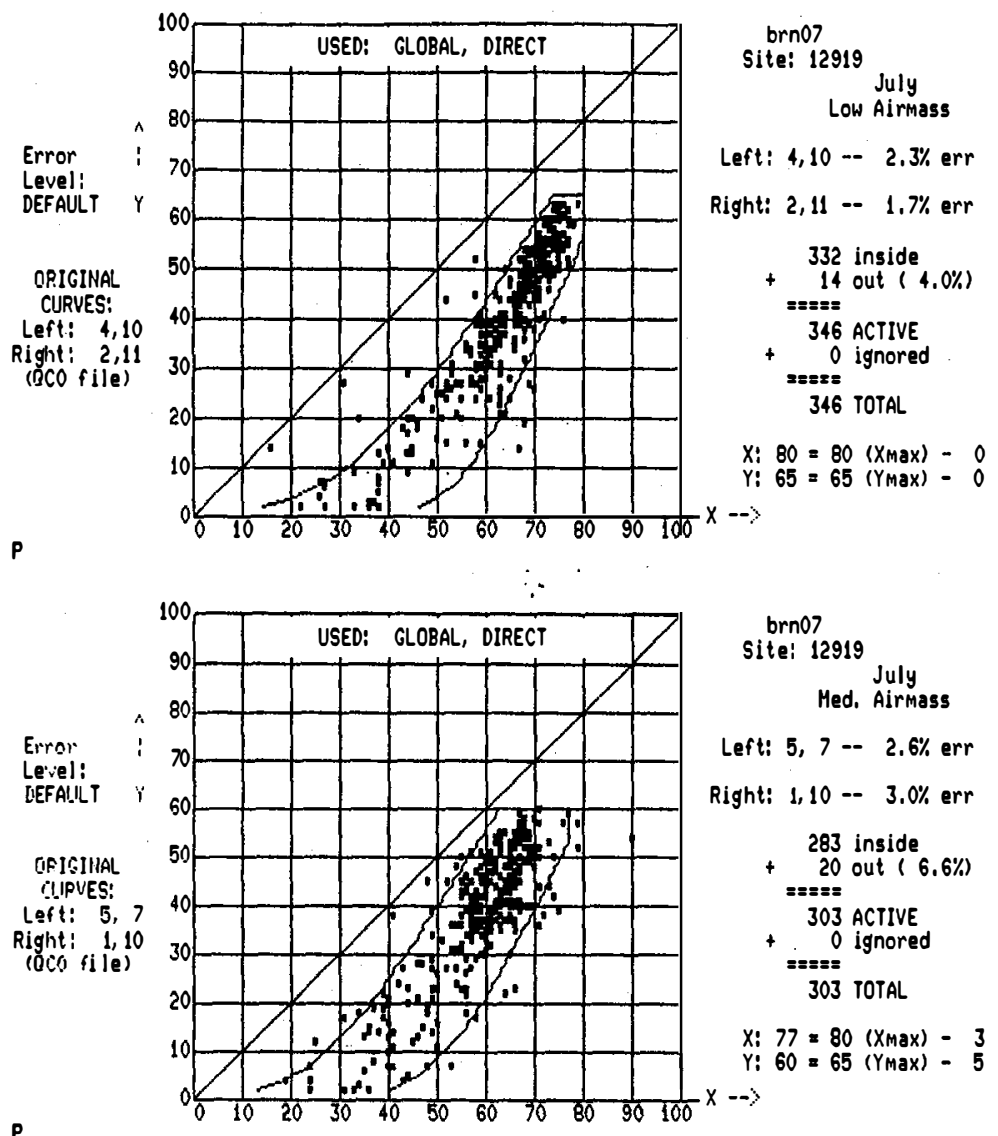

家 p

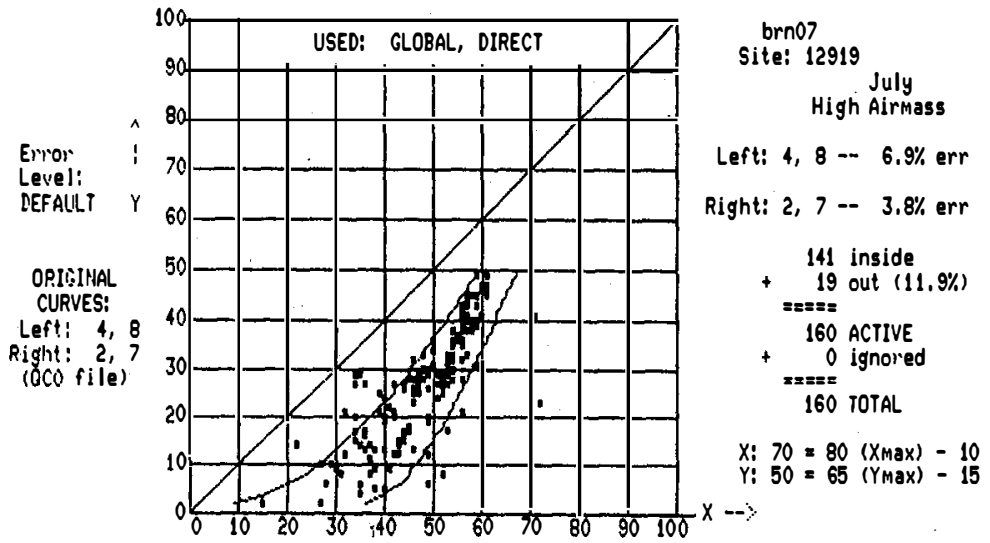

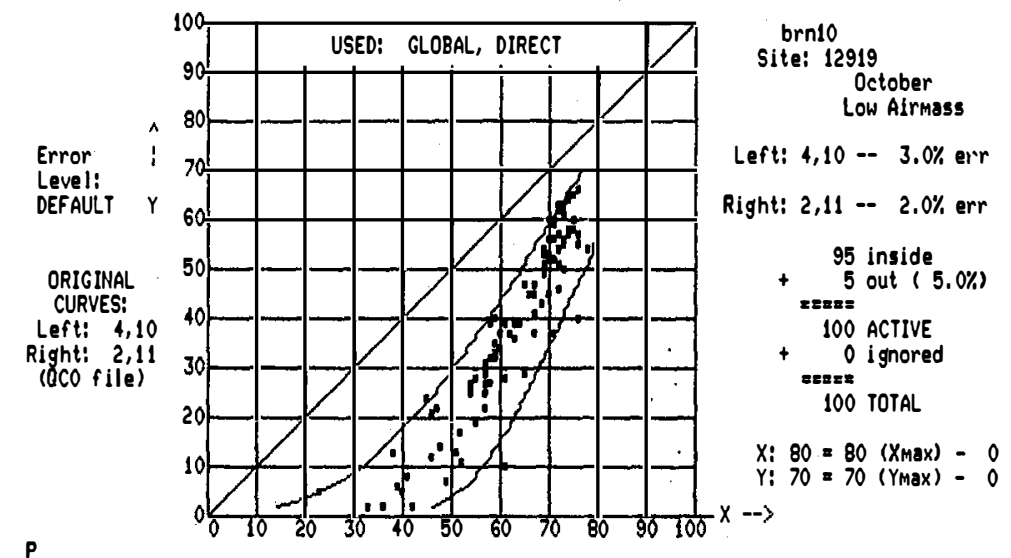
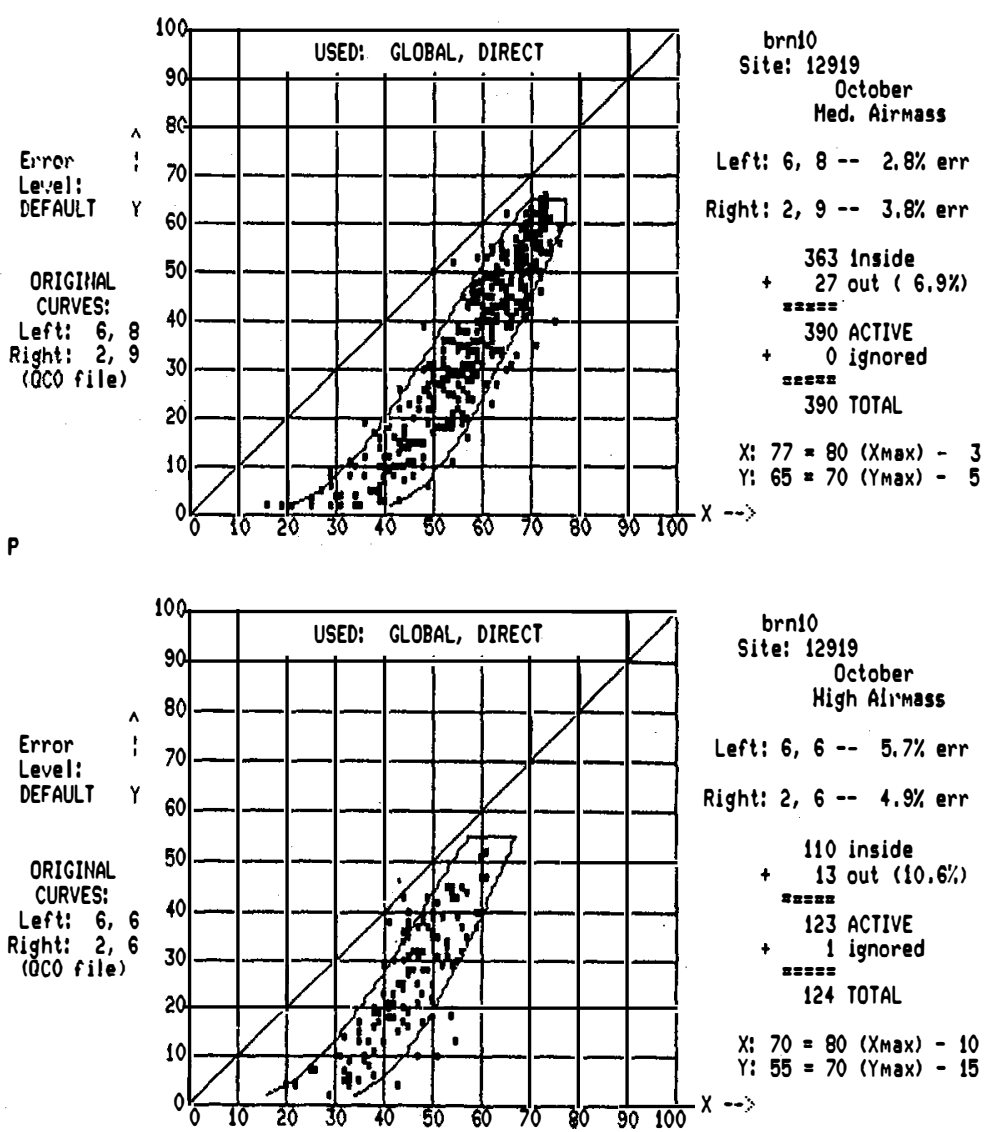

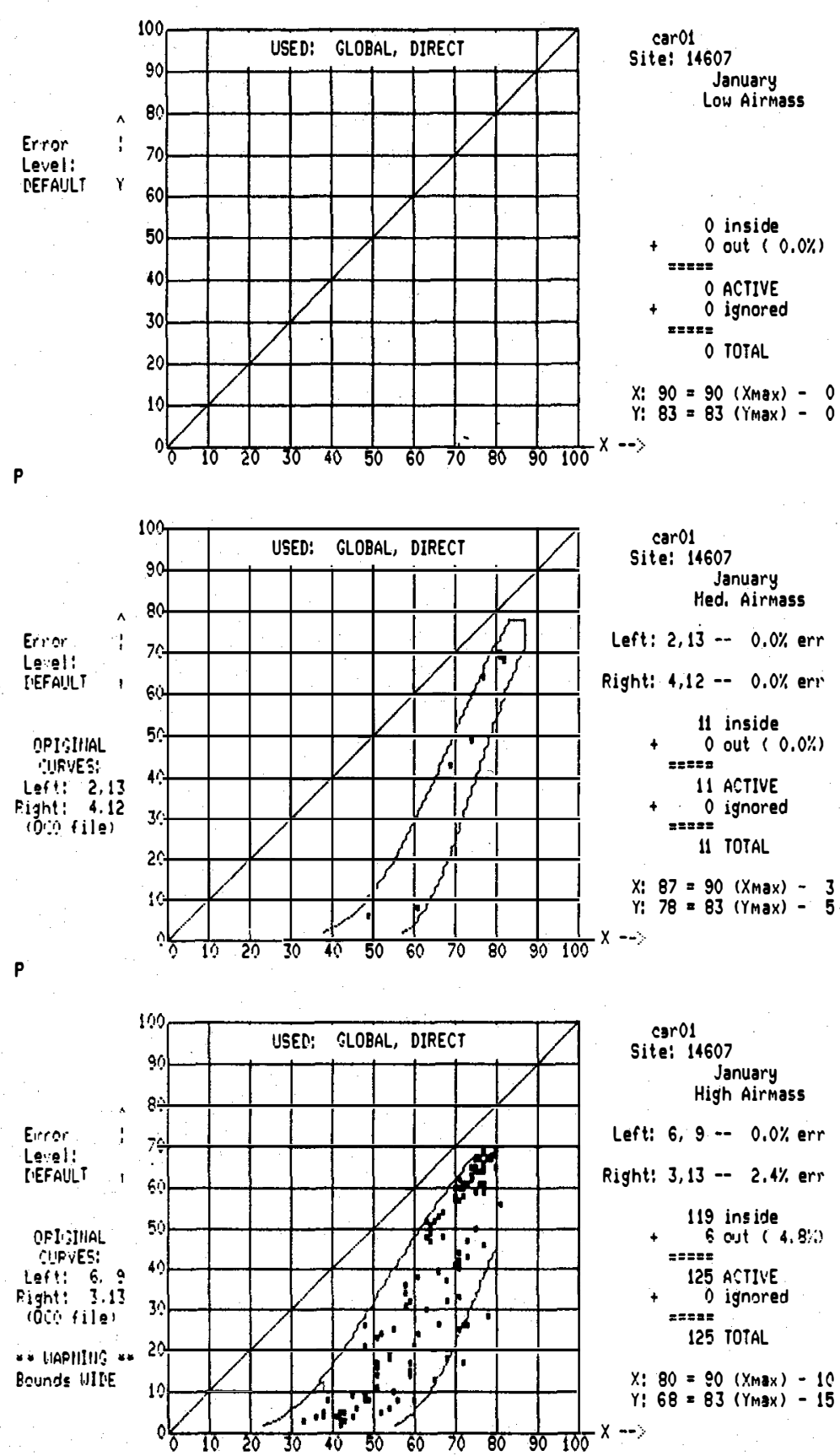
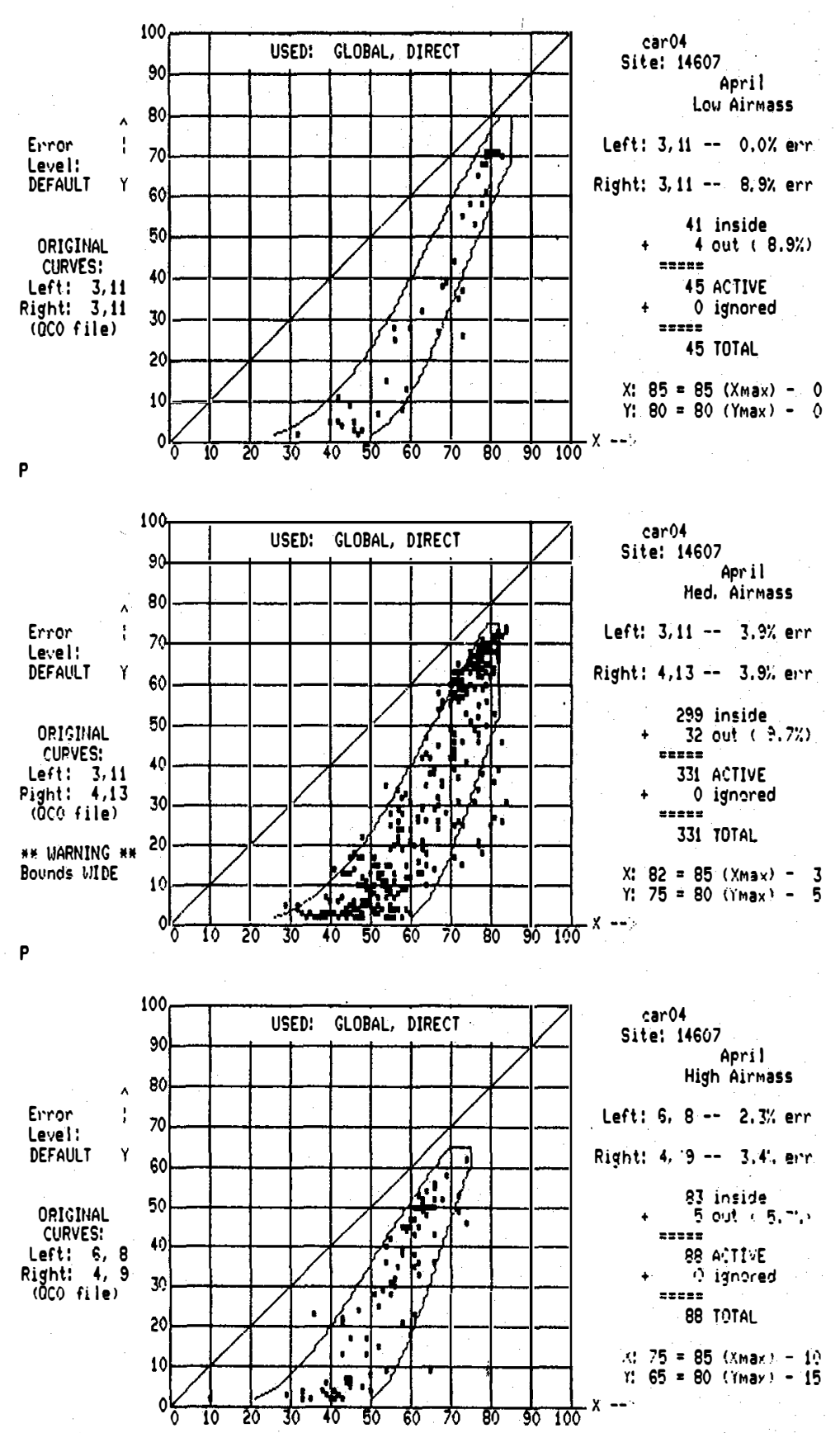

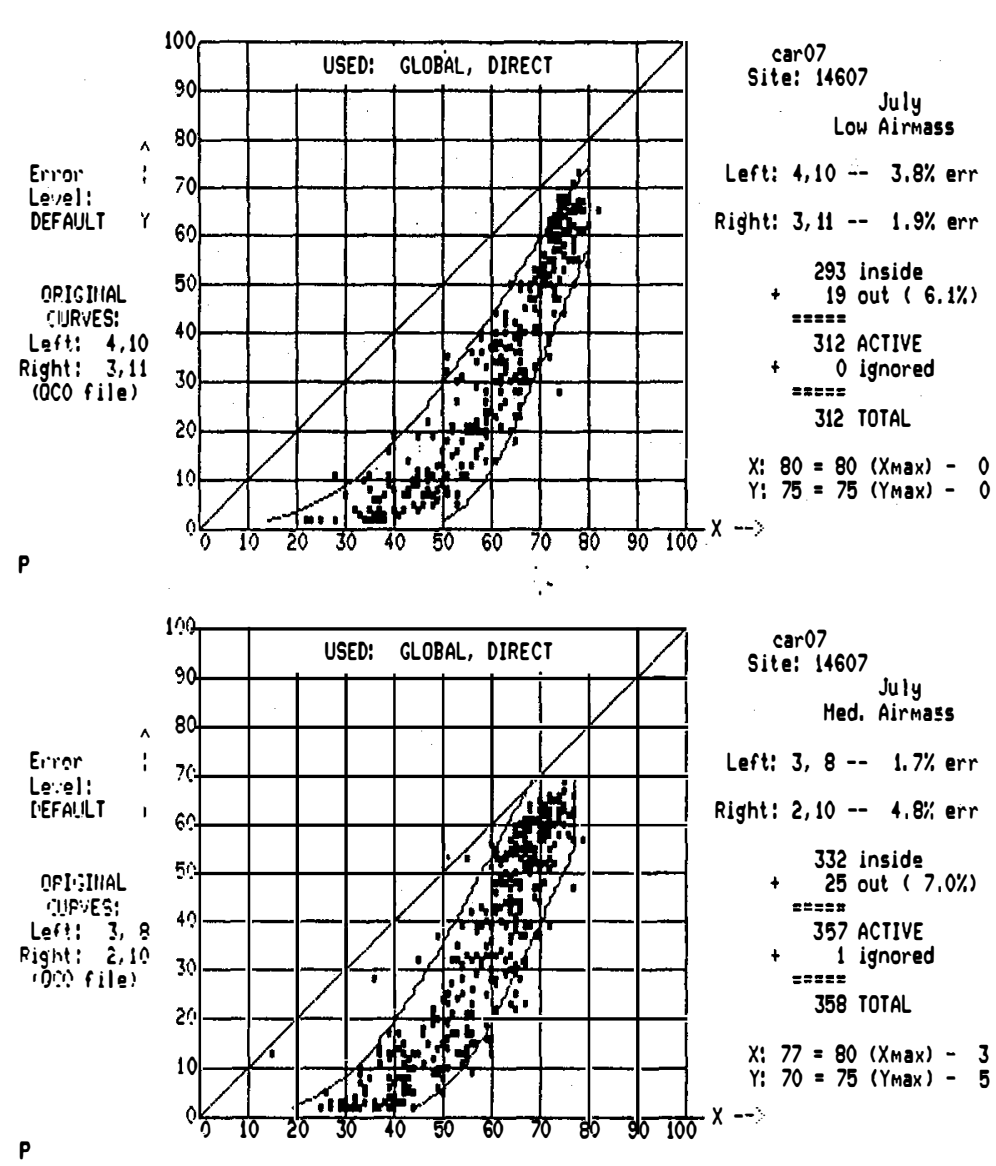

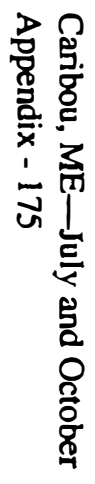
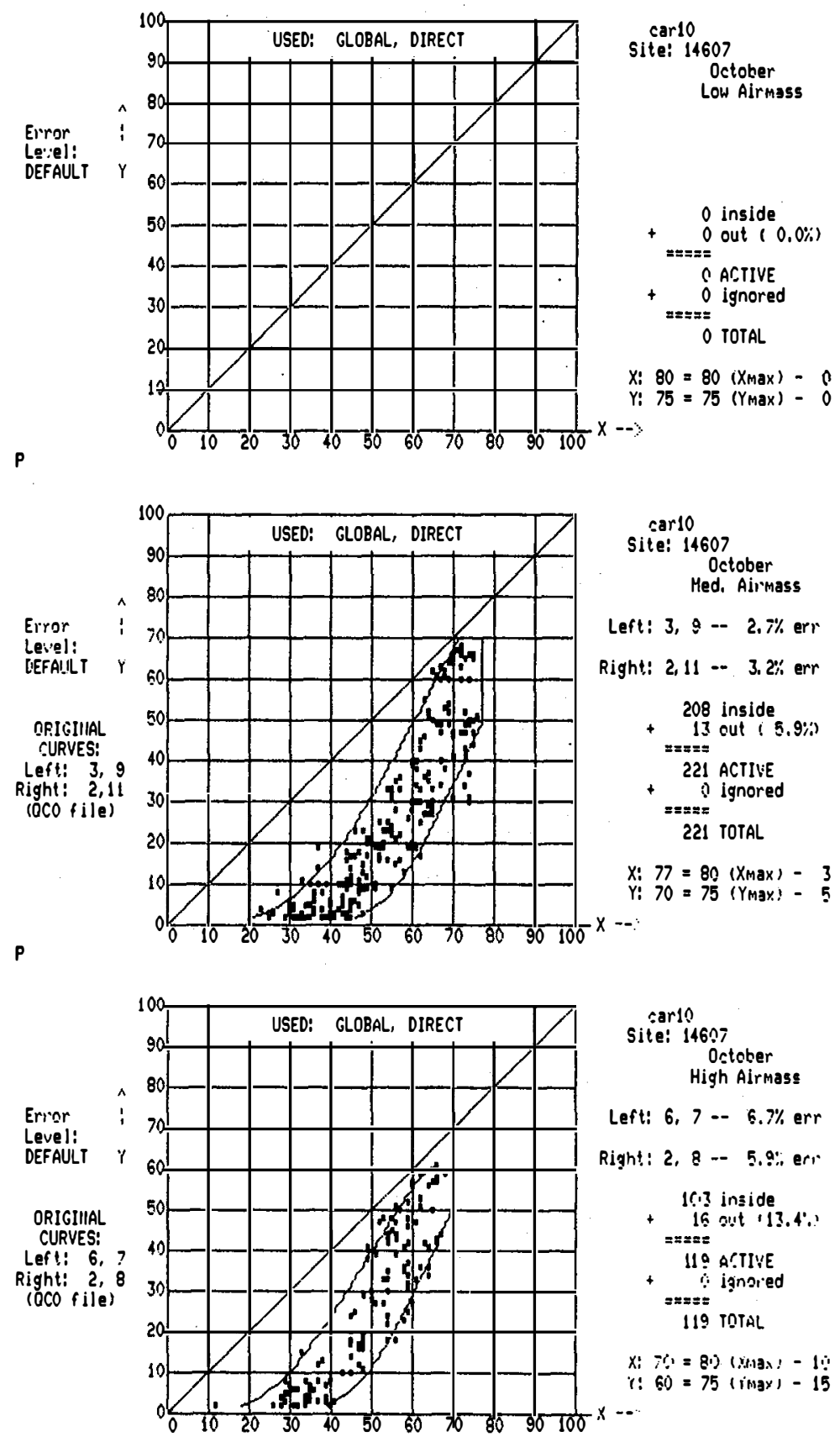


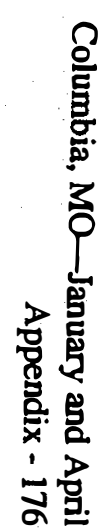

$\mathbf{p}$

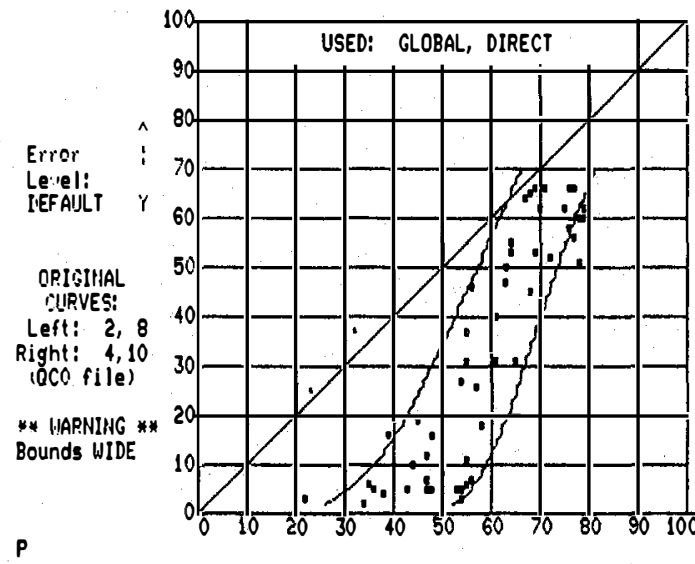
Sol01 January Left: $2,8--4.0 \% \mathrm{err}$ Right: $4,10--10.0 \%$ err
$\quad 43$ inside
$+\quad 7$ out $(14.0 \%)$ $== \pm=$ 50 ACrive
$+\quad 2$ ignored 52 TOTAL

$X: 82=85\left(X_{\text {max }}\right)-3$
$Y: 70=75\left(Y_{\text {max }}\right)-3$ Y: $70=75\left(Y_{\text {max }}\right)-5$ $X: 85=85\left(X_{\max }\right)-0$
$Y: 75=75\left(Y_{\max }\right)-0$
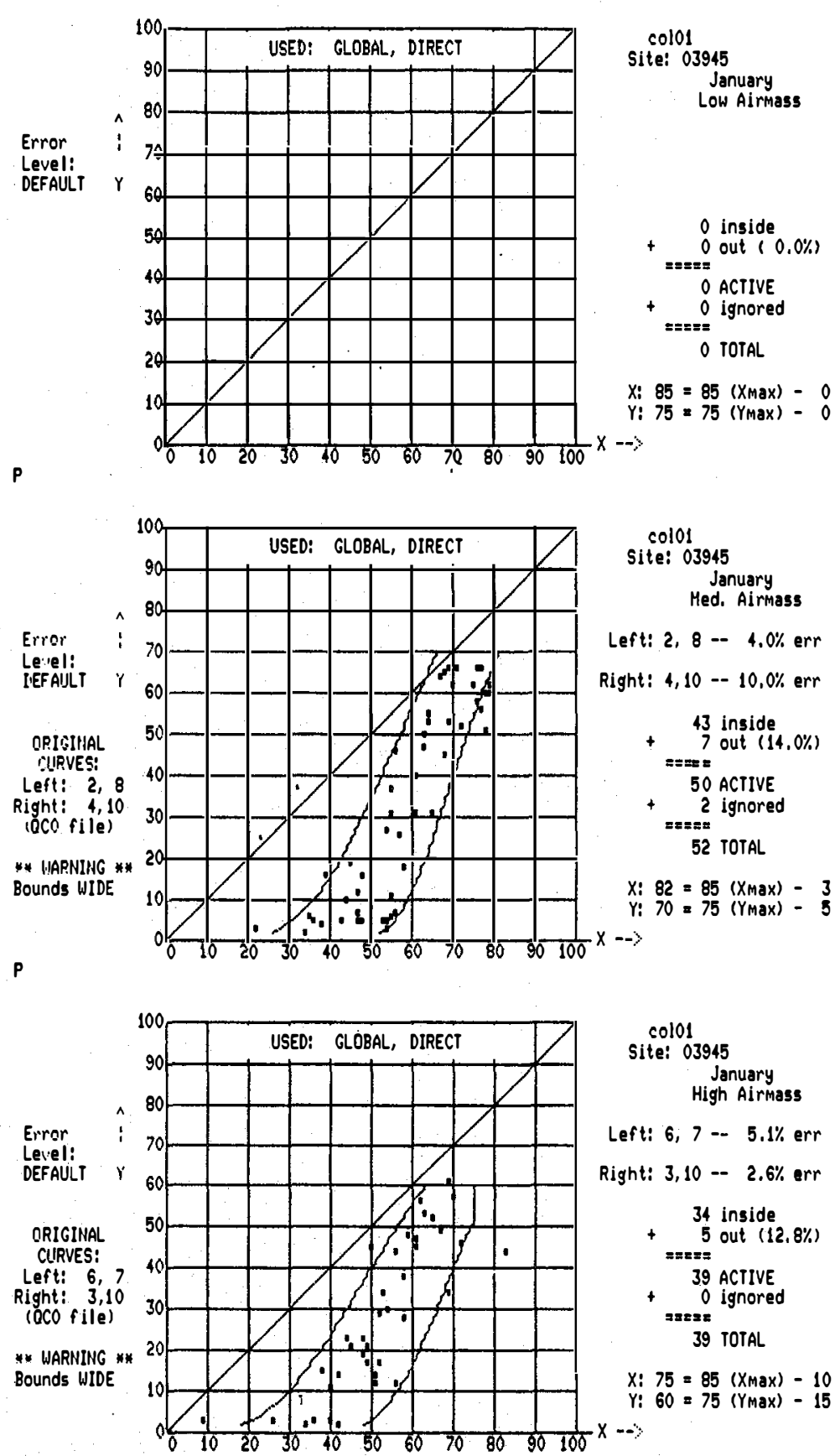
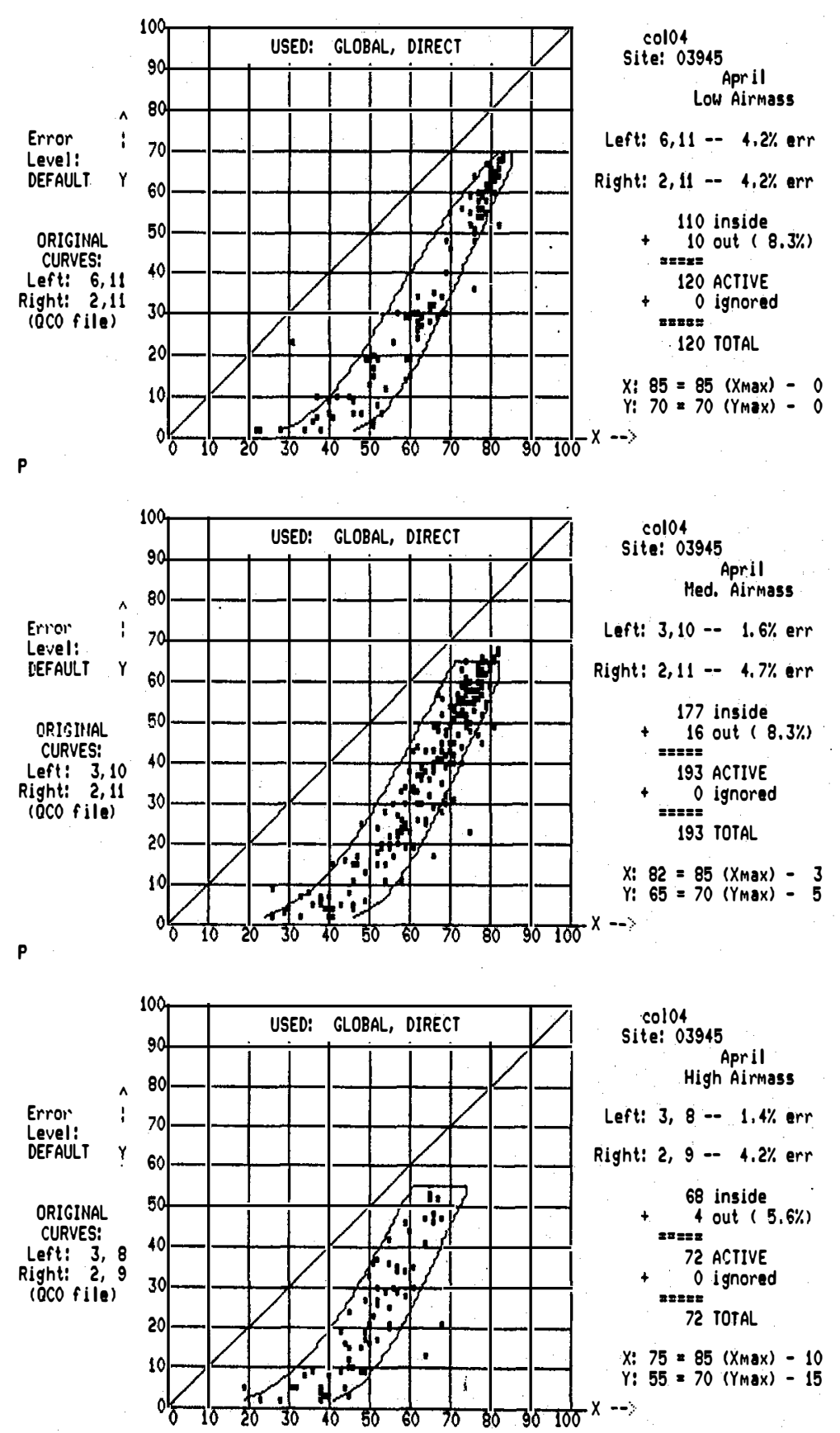

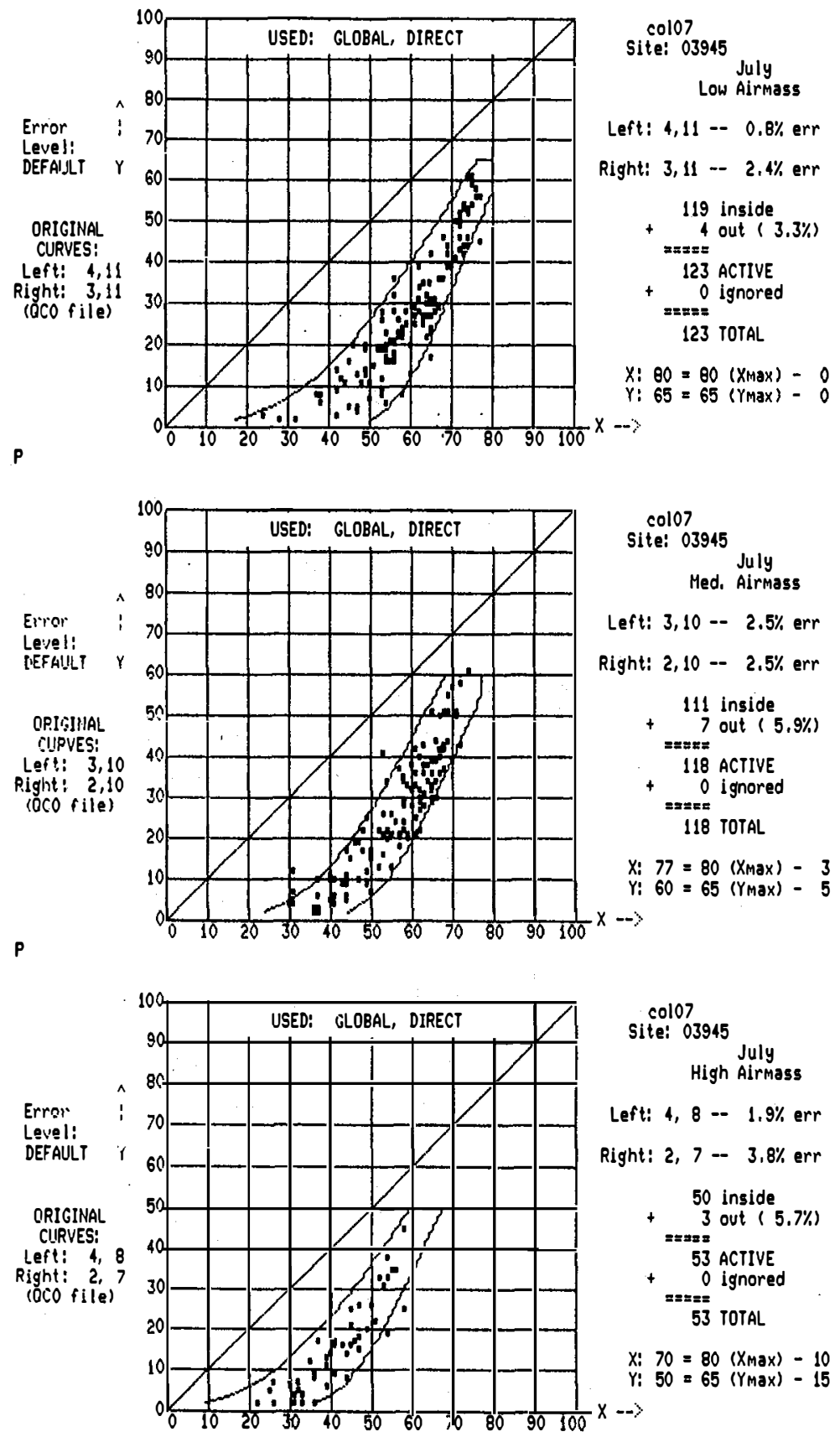
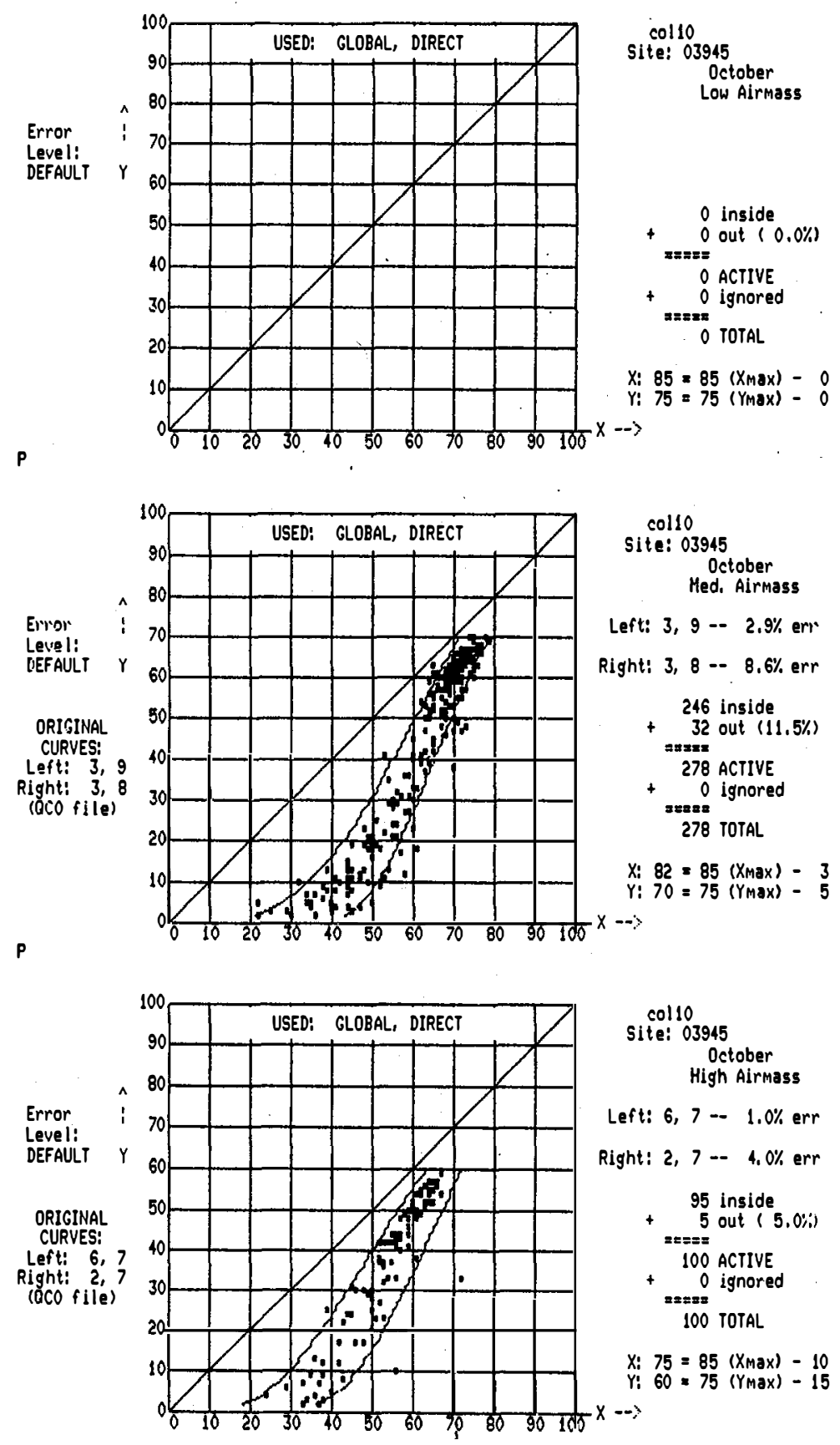

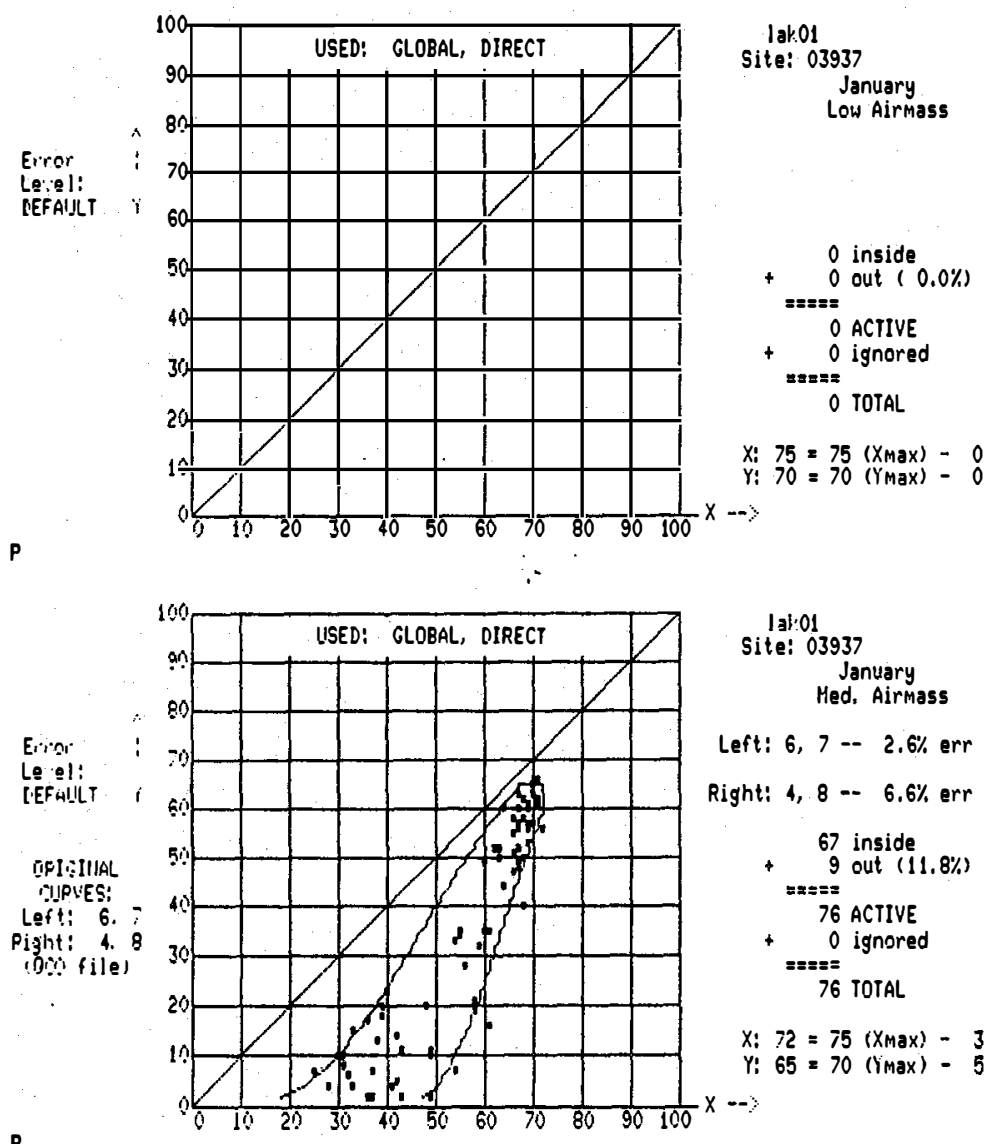

Site: 013937 January
Med, Airmass Left: $6,7-2.6 \% \mathrm{err}$ Right: 4, 8 -- $6.6 \%$ err $\quad 67$ inside
$+\quad 9$ out $(11.8 \%)$ $=z===0$ out$$
+\quad 0 \text { ignored }
$$$$
76 \text { TOTAL }
$$

$x: 72=75\left(x_{\max }\right)-3$ $Y: 65=70($ ('max $)-5$

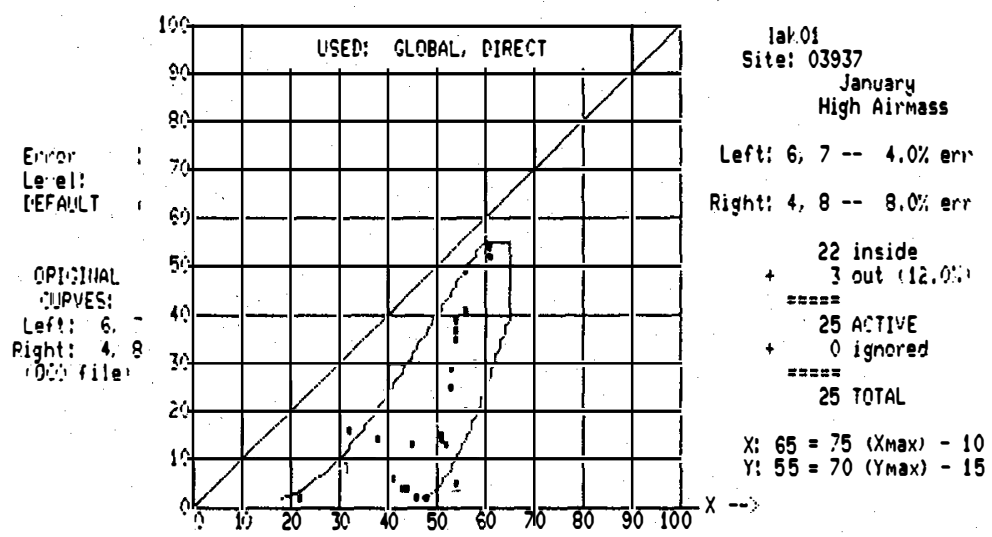

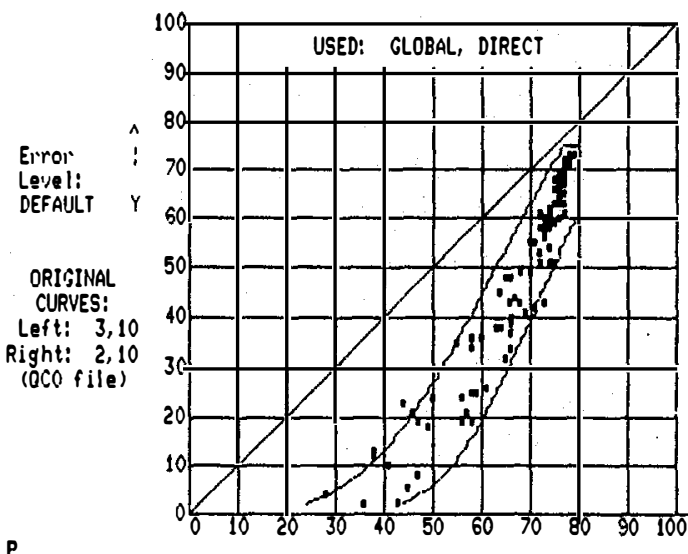

Site: 03903 Site: 03937 April
Low Airmass Left: $3,10-2$ - $4.1 \%$ err Right: 2,10 -- $1.0 \%$ err

93 inside
$+\quad 5$ out $(5,1 \%)$

$== \pm=$

98
0 ignored

98 TDTAL

X: $\begin{aligned} & 80=80\left(X_{\text {max }}\right)=0 \\ & Y: 75=75\left(Y_{\text {max }}\right)-8\end{aligned}$
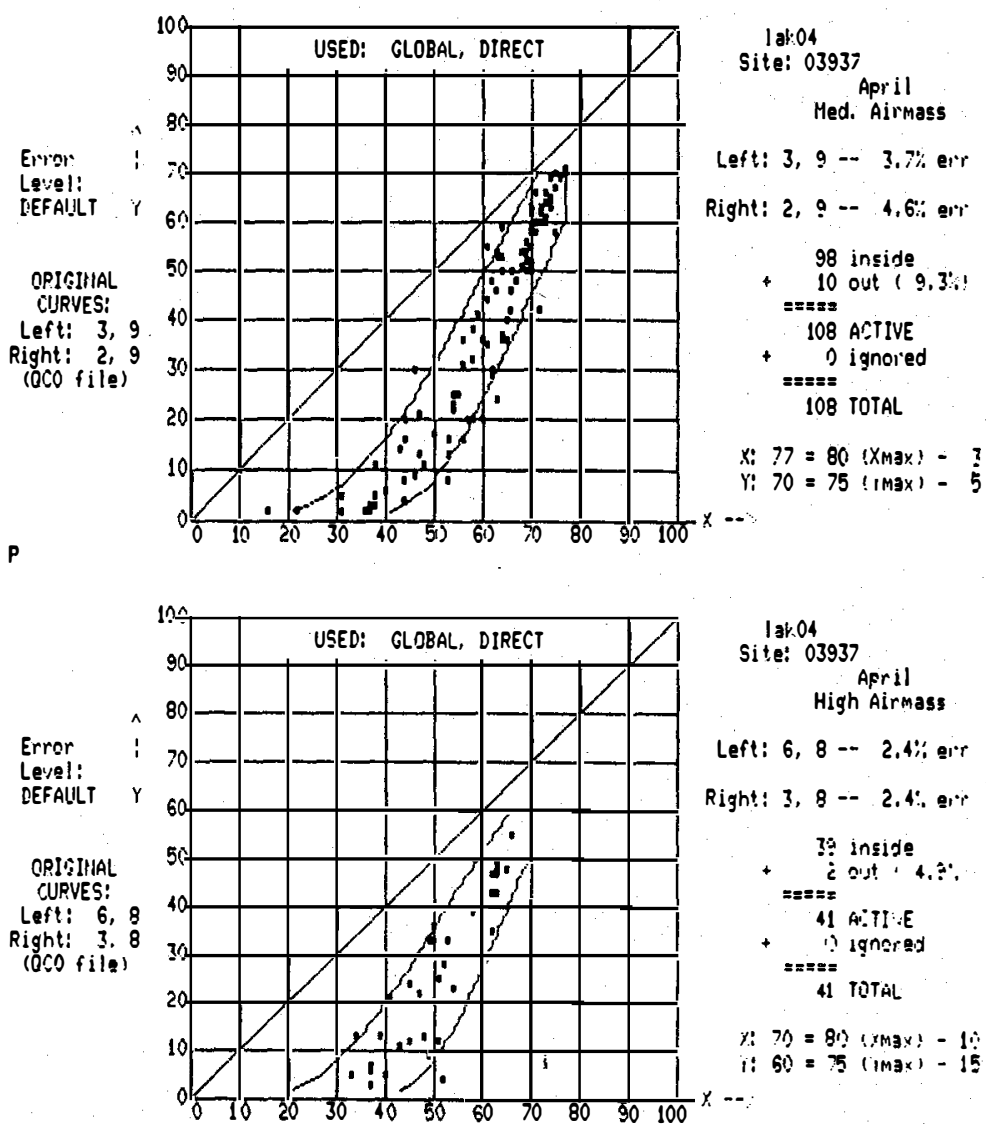

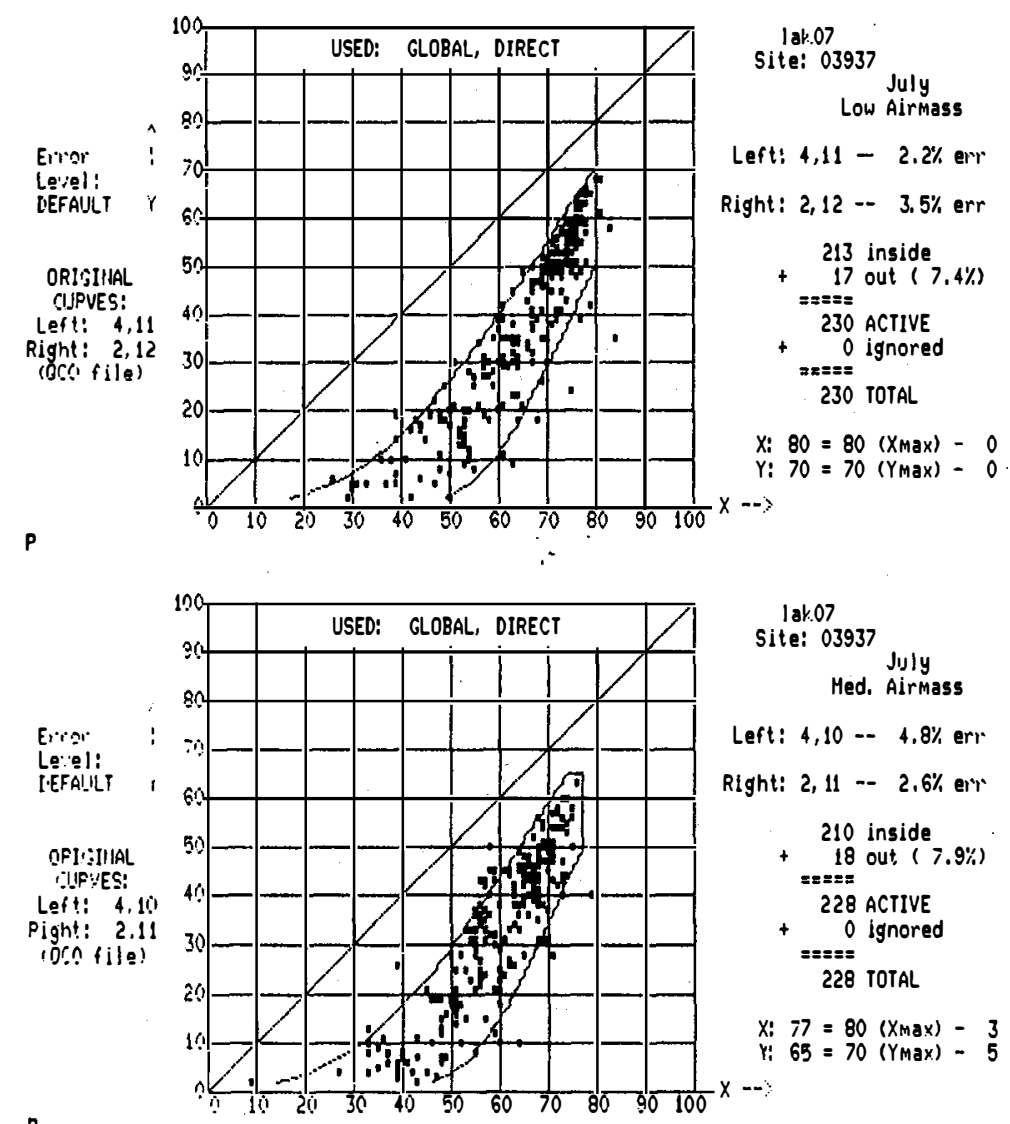

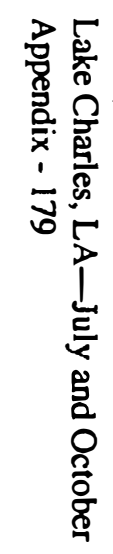

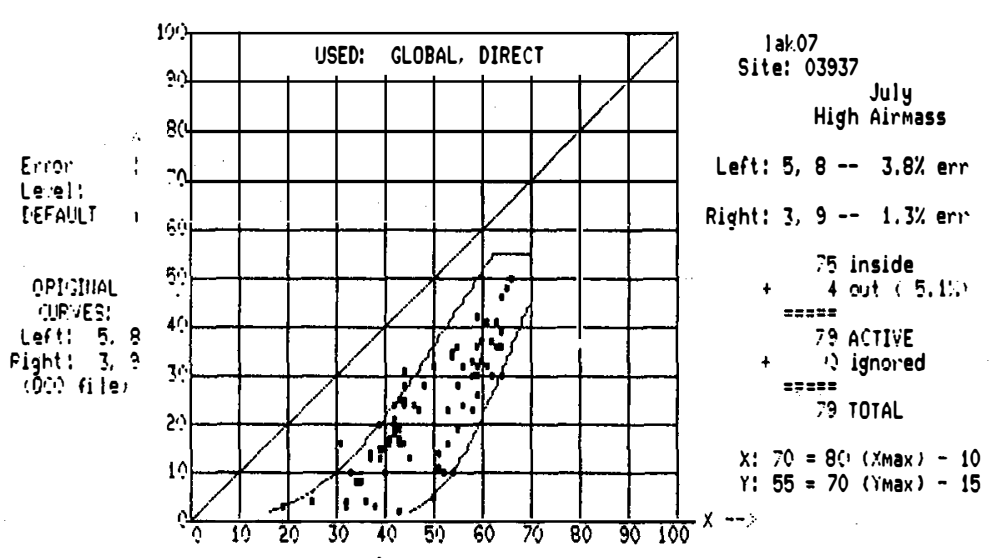

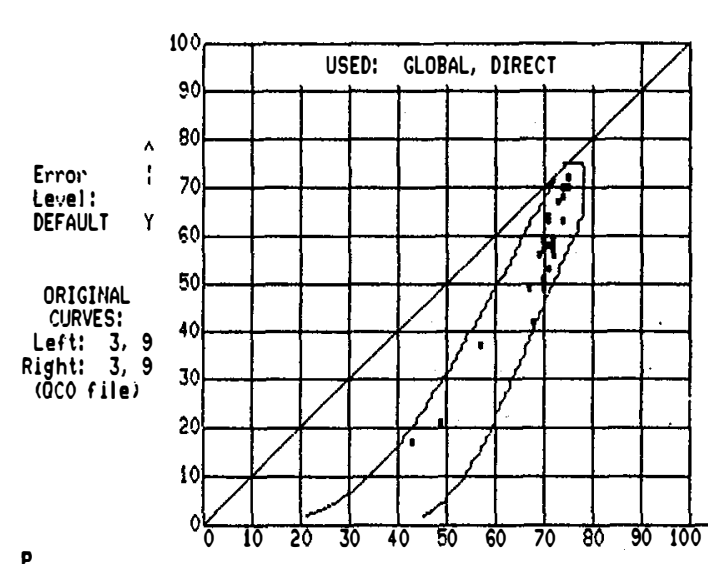
lab.10
Site: 03937 Qctober
Low Airsas

Left: $3,9--0.0 \%$ err

Right: $3,9-0.0 \% \mathrm{err}$

$\quad 32$ inside
$+\quad 0$ out $(0.0 \%)$

$== \pm=$

32 ACTIVE
$+\quad 0$ ignoied

$== \pm 2$ TOTAL

$X_{:} 78=78\left(X_{\text {max }}\right)-0$
$Y_{:} 75=75\left(Y_{\max }\right)-0$

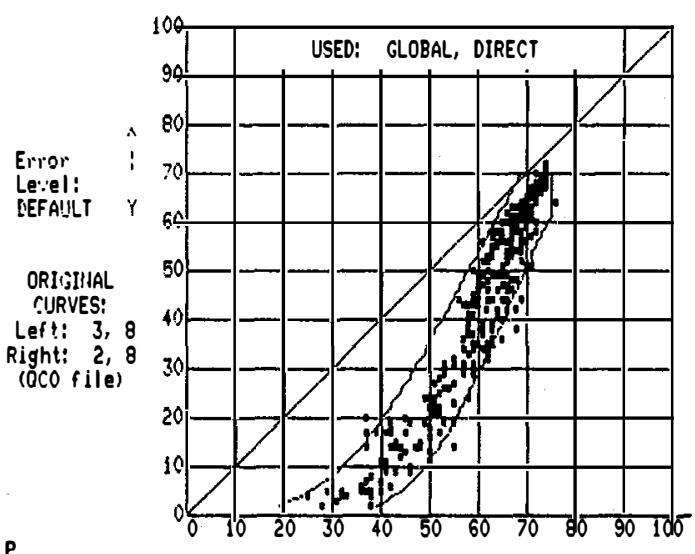

lat 10
Site: 03937 Ostoher Hed. Airmass

Left: 3, 8 -- 0.6:i: err Right: 2, 8 - 2.4\% err

$\begin{aligned} & 325 \\ + & 13 \text { inside } \\ + & \text { ost }(3,8 \%)\end{aligned}$

338 ACTIVE

$+\quad 0$ ignored

338 TOTAL

$X_{i} 75=78$ (Y.Max) - 3 if $70=75\left({ }^{\prime}\right.$ Max $)-5$

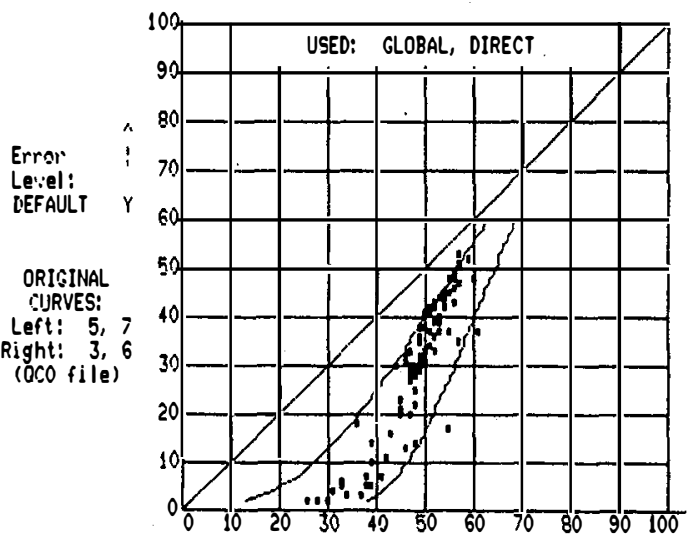

191.10 Ostober
Hig̣h Airmass

Left: $5,7-2.3 \% \mathrm{err}$ Right: 3. $6-2.3 \%$ err

83 inside
$+\quad 4$ zut 1 $4.6^{\circ}$,

+ 4 aut 14.6

8. AITIVE

1) Iรngi"ed

87 total $x: 68=78$ (Ymax) -10
$60=75$ (Ymax) -15 


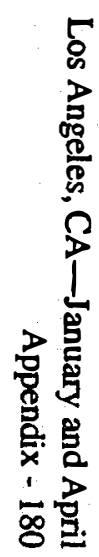

Error Level:
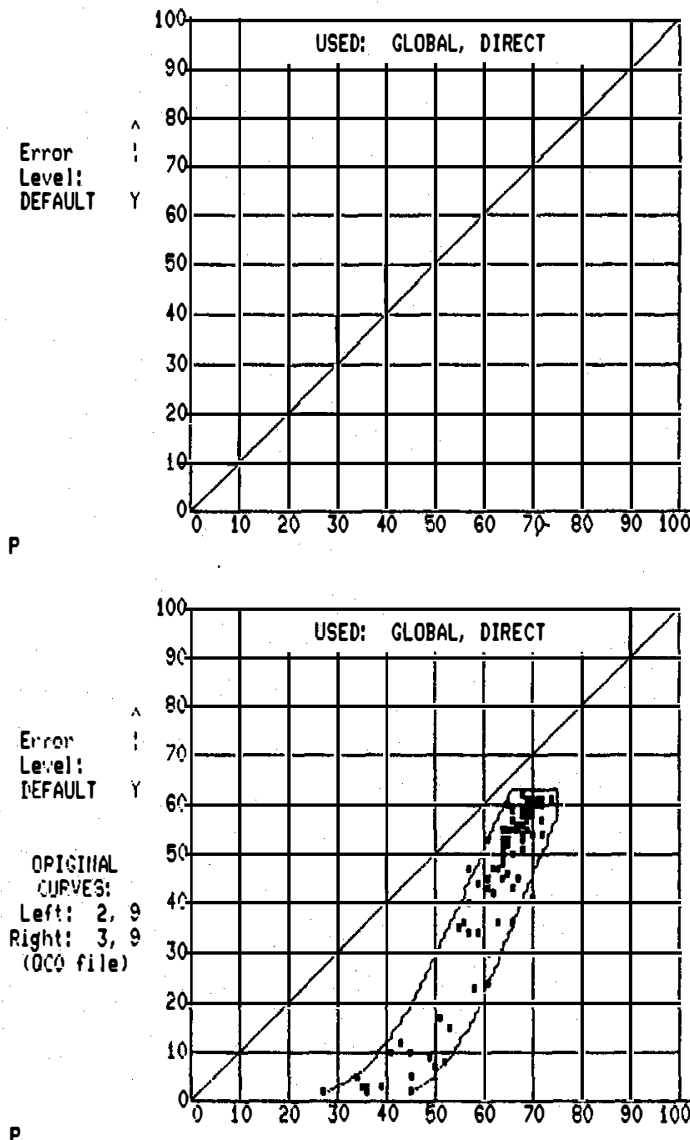

Site: 2317

January Med. Airmass

0 inside
$+\quad 0$ out $(0.0 \%)$

0 ACTIVE

0 ignored

0 TOTAL

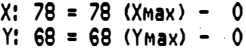

$\rightarrow$

Left: $2,9--2.1 \%$ er

Right: $3,9--1.0 \% \mathrm{err}$

$\begin{array}{r}93 \text { inside } \\ +\quad 3 \text { out }(3.1 \%) \\ \hline\end{array}$

96 ACTIVE

0 ignored

$=26$ TOTAL

$X: 75=78\left(X_{\max }\right)-3$
$Y: 63=68\left(Y_{\max }\right)-5$ $\rightarrow$

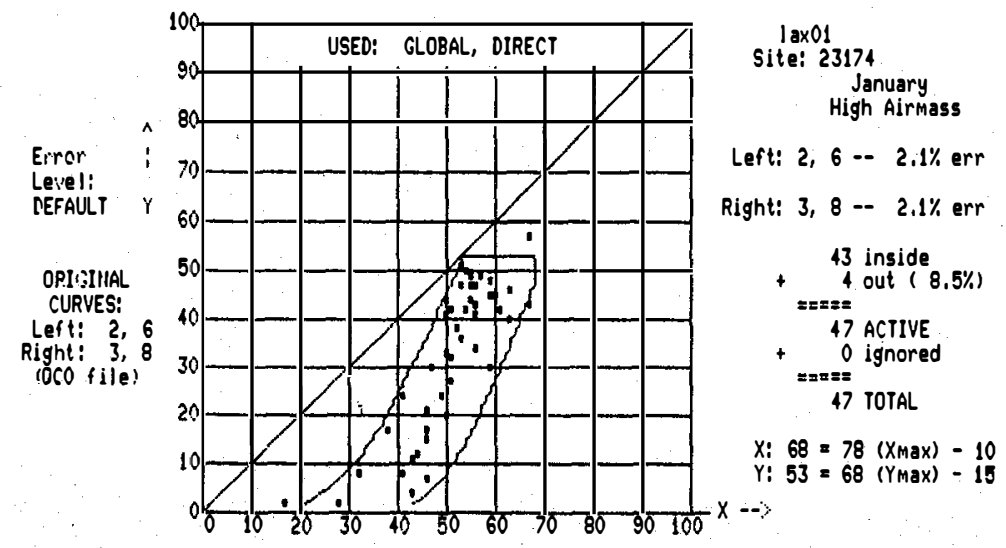

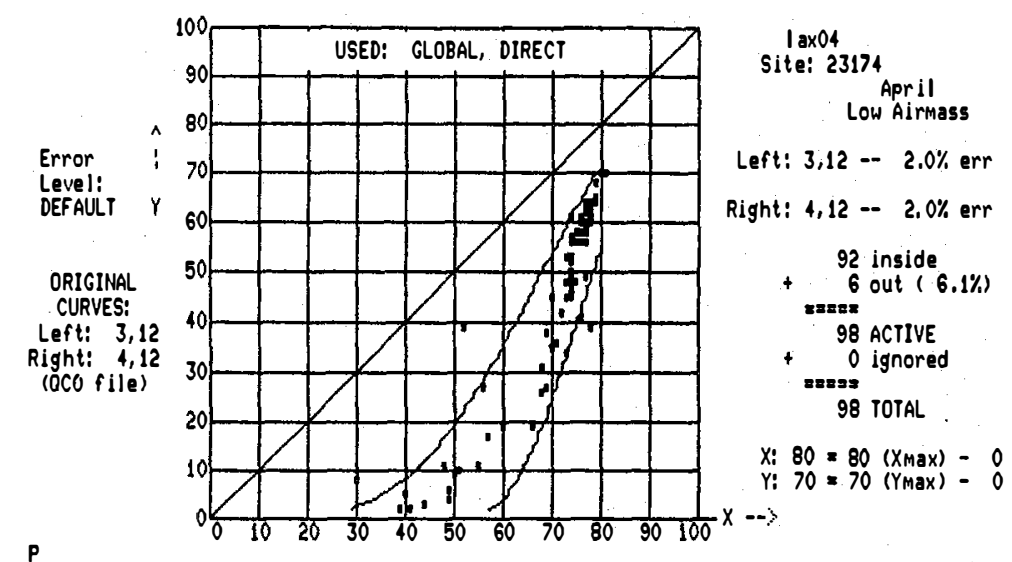
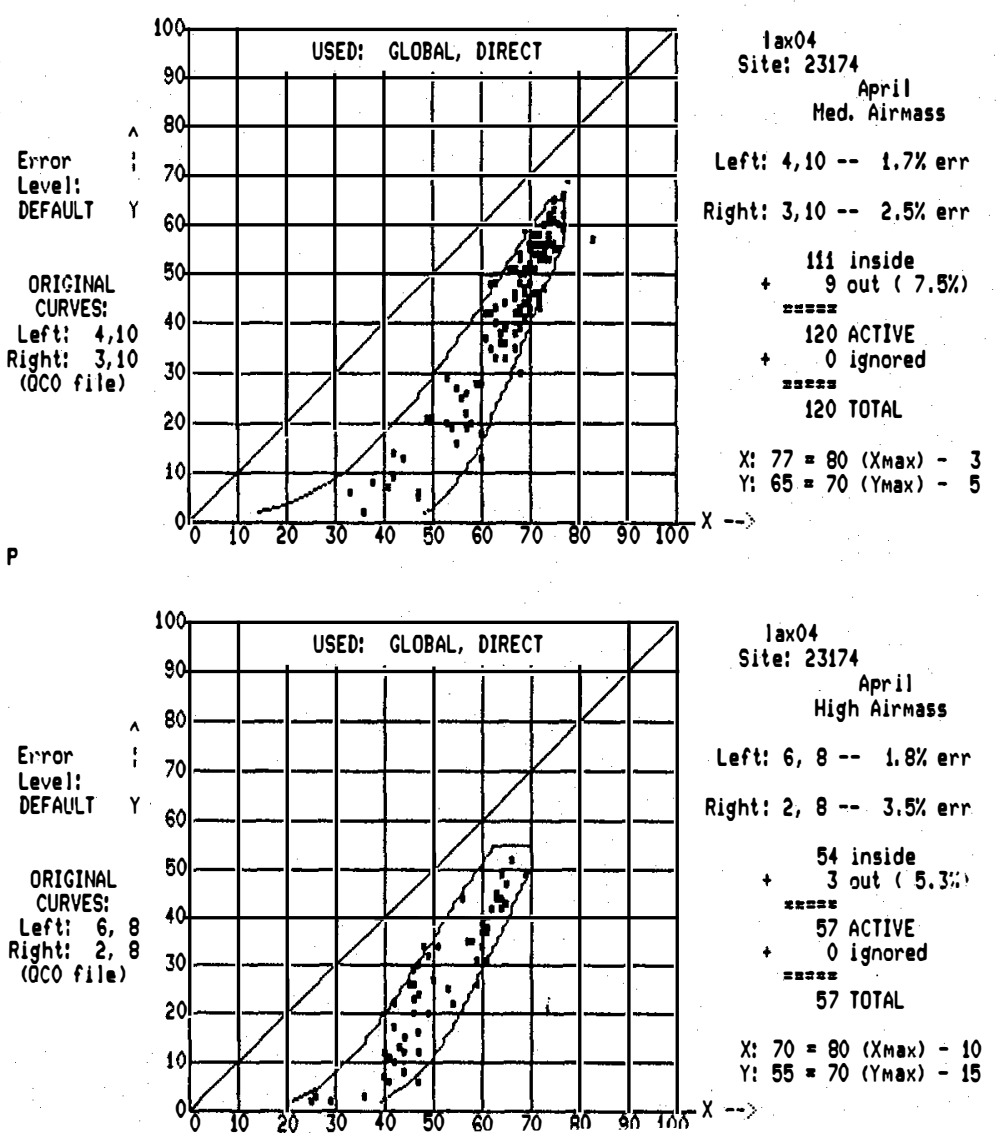

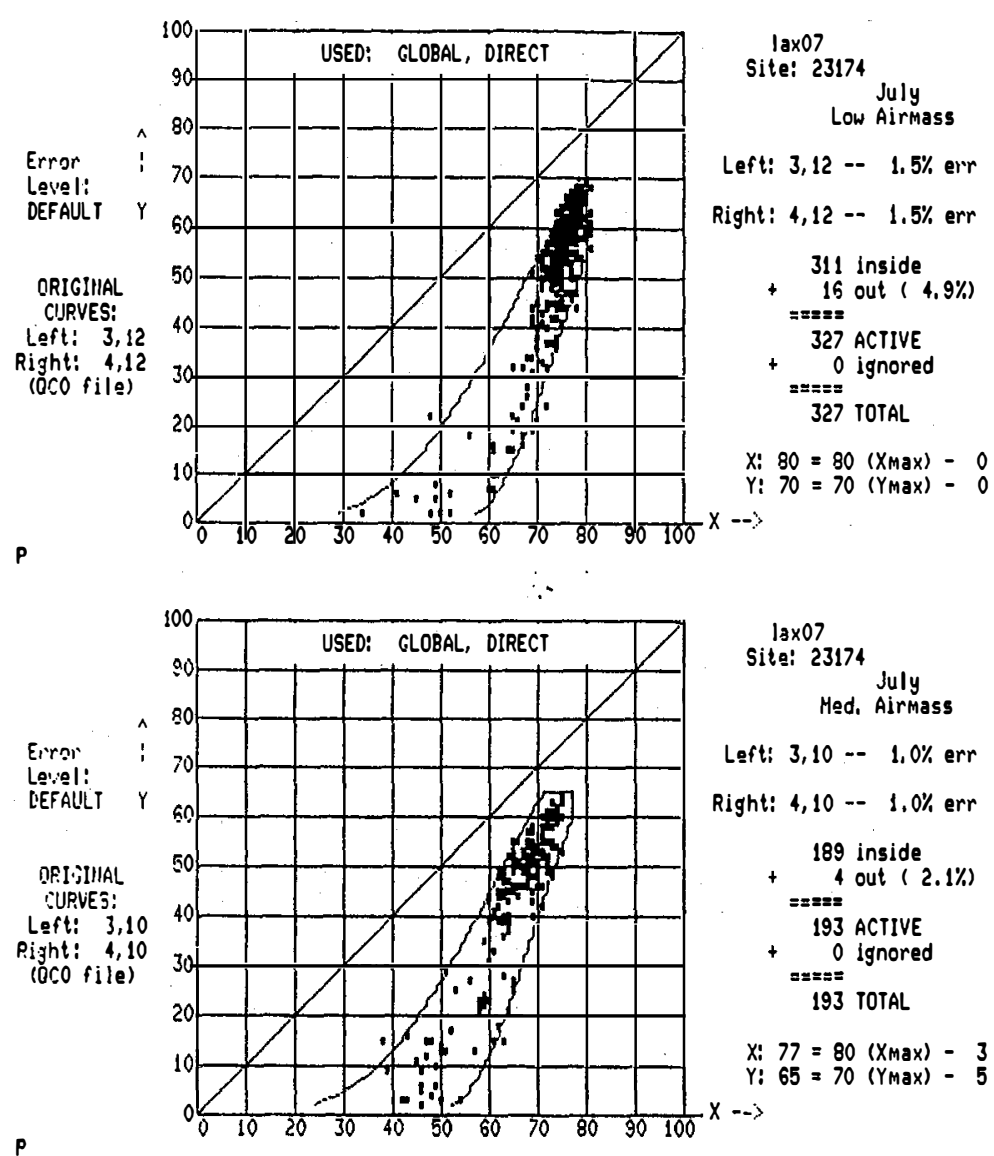

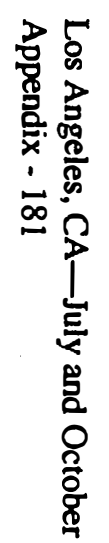
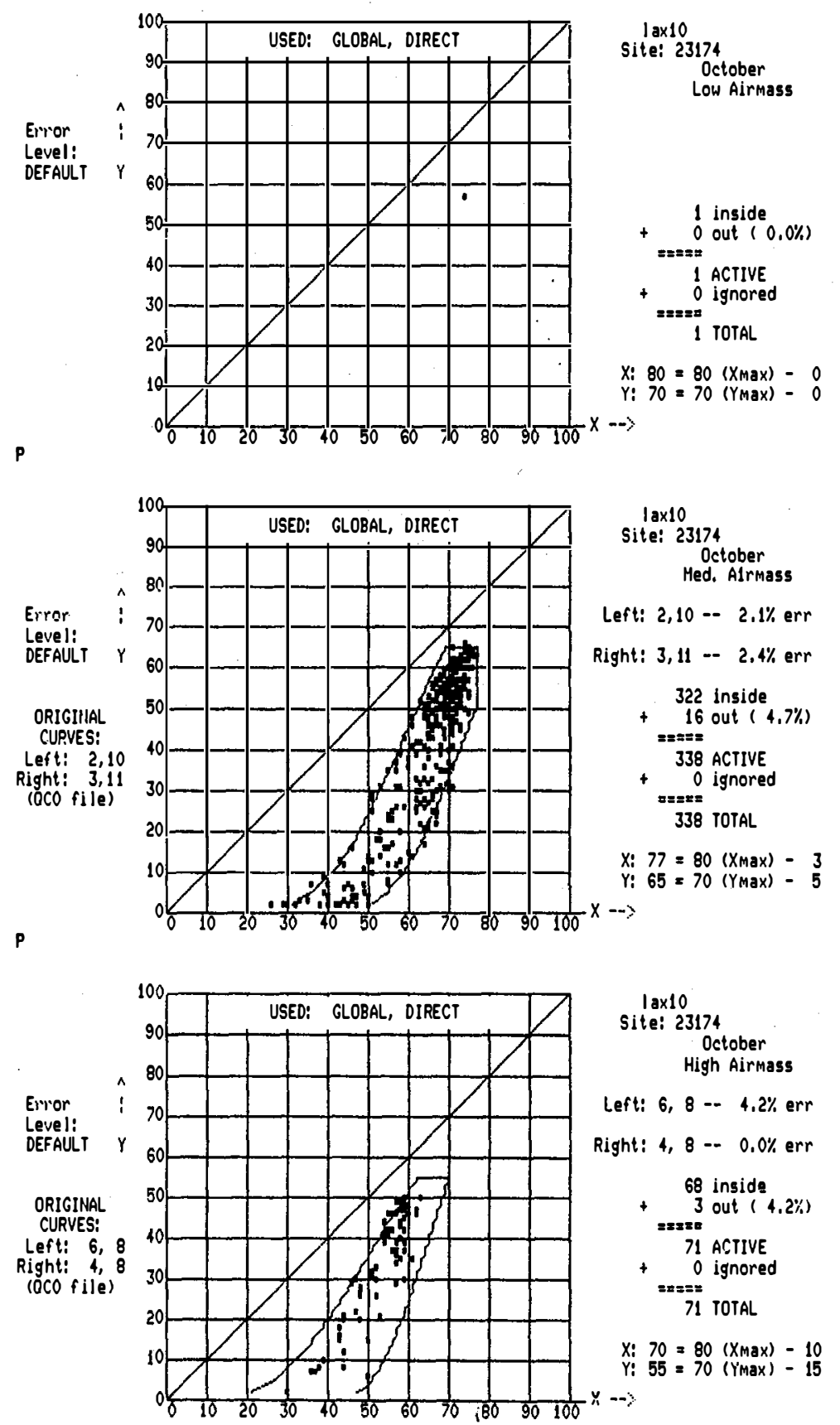
$\mathbf{p}$

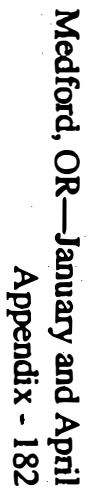

Error

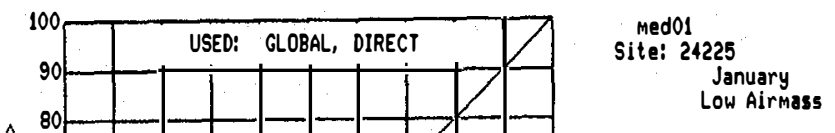

Level:

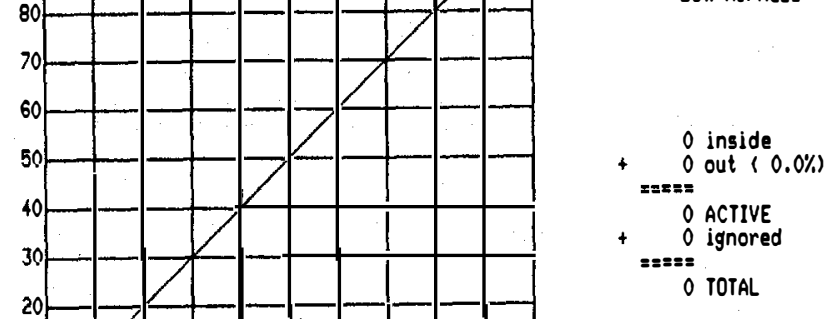

20

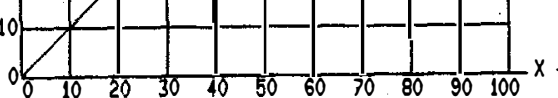

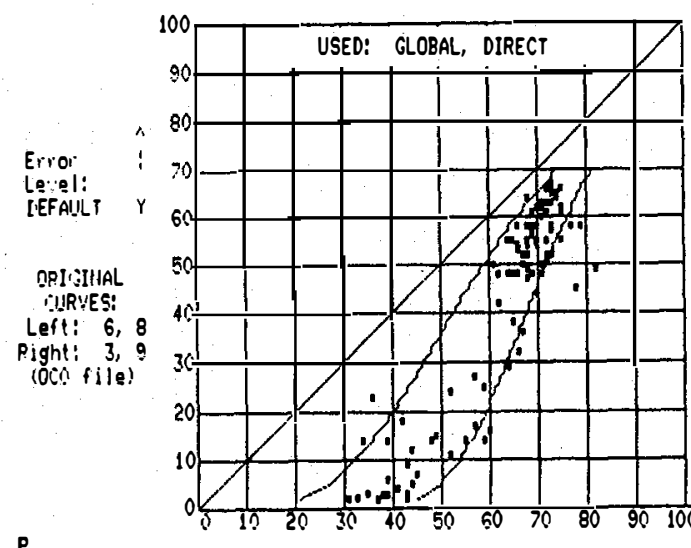

Site: 2422

January Med. Airmass

Left: $6,8-3.2 \%$ err

Right: 3, $9--11,7 \% \mathrm{err}$

$\begin{aligned} & 80 \text { inside } \\ &+\quad 14 \text { out (14.9\%) }\end{aligned}$

94 ACTIVE

$+\quad 0$ ignored

94 TOTAL

Y: $82=85\left(\right.$ Xax $_{\text {Max }}-3$
Y: $70=75\left(Y_{\text {max }}-5\right.$

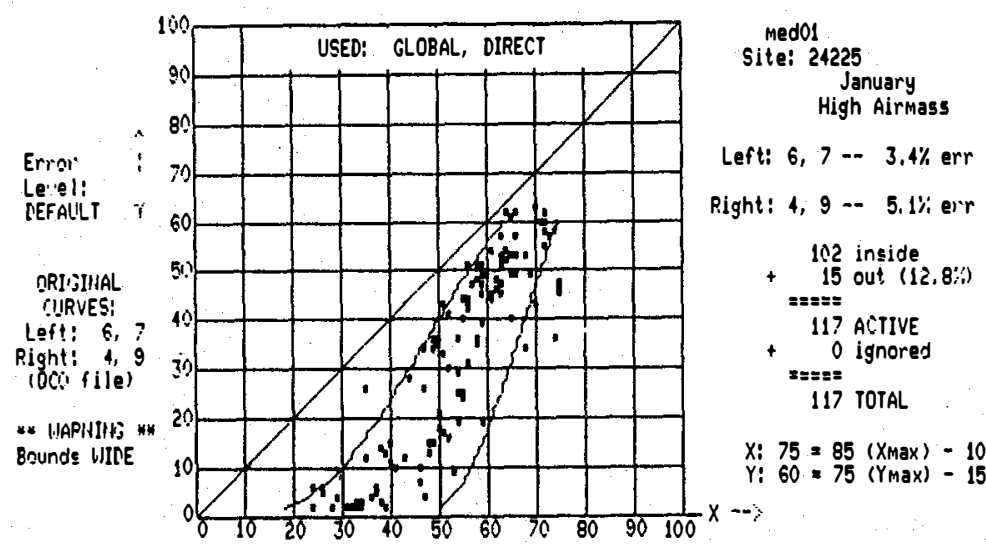

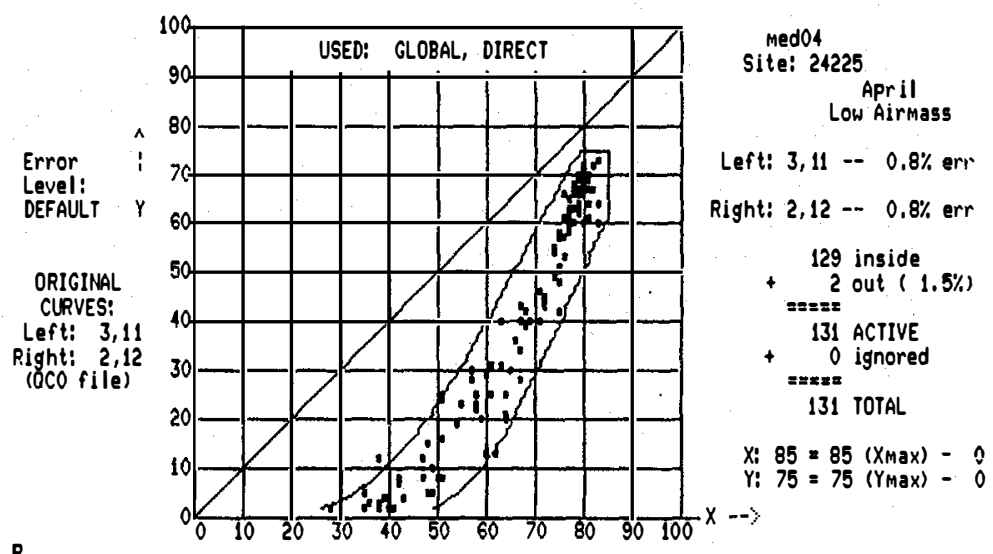
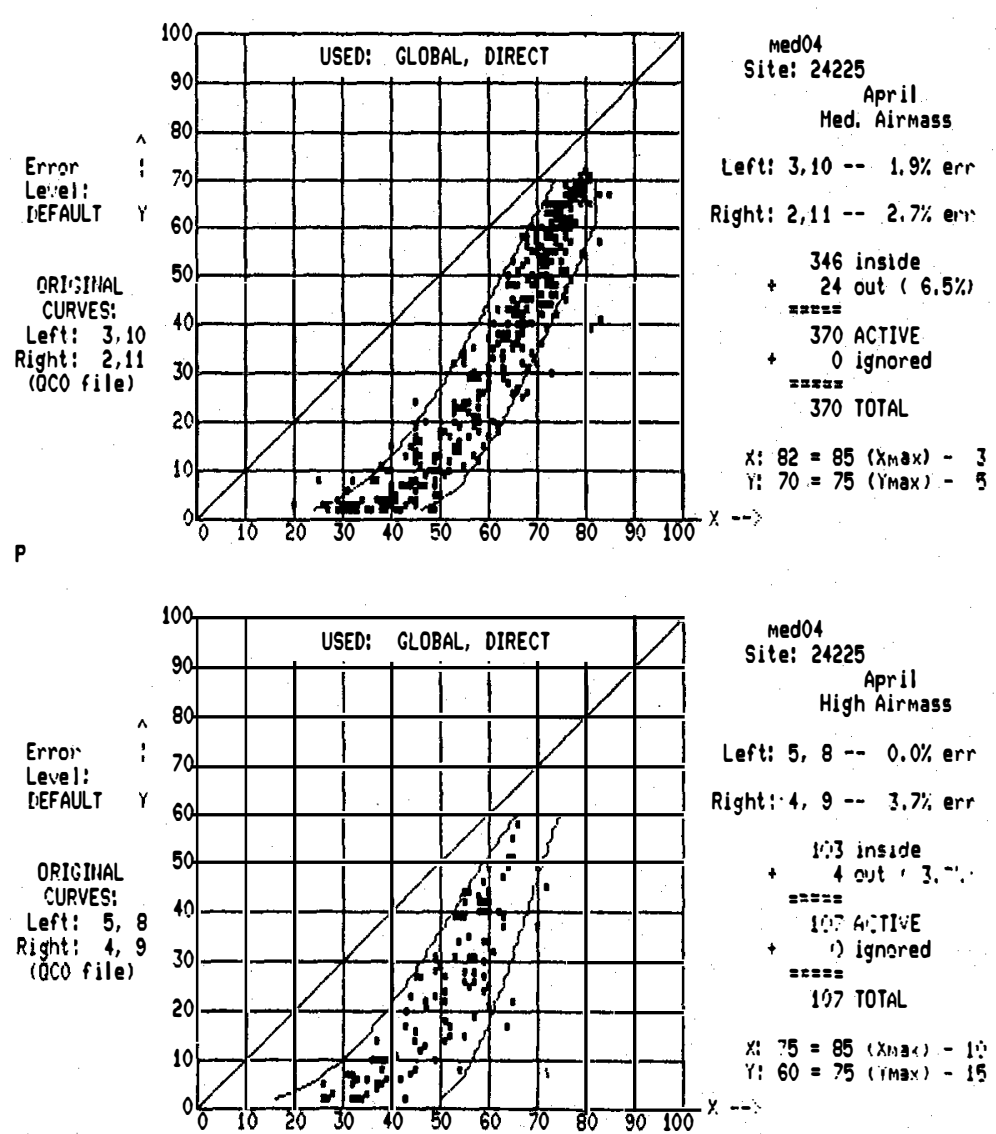

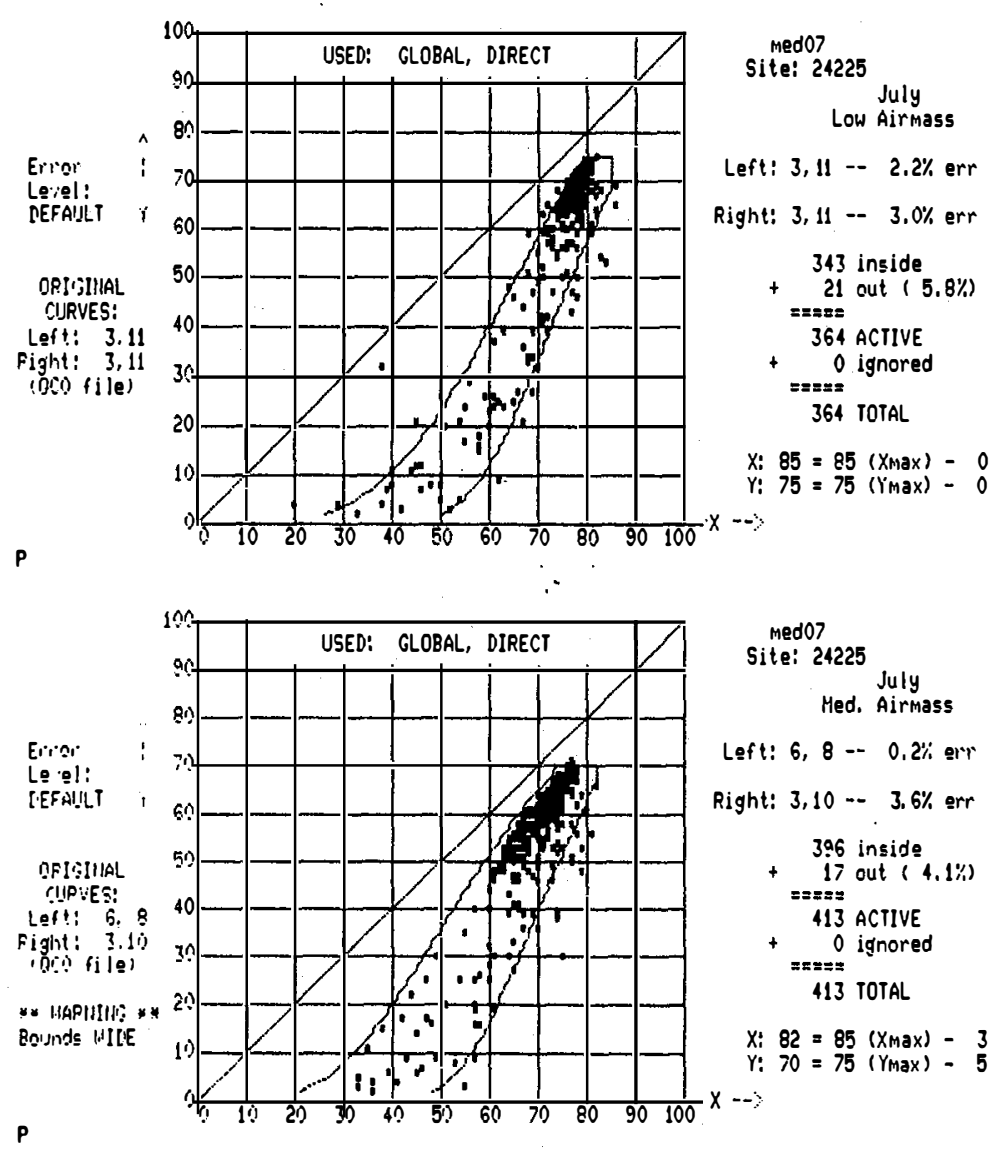

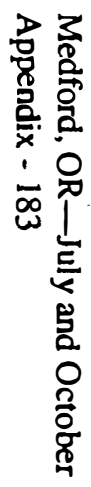
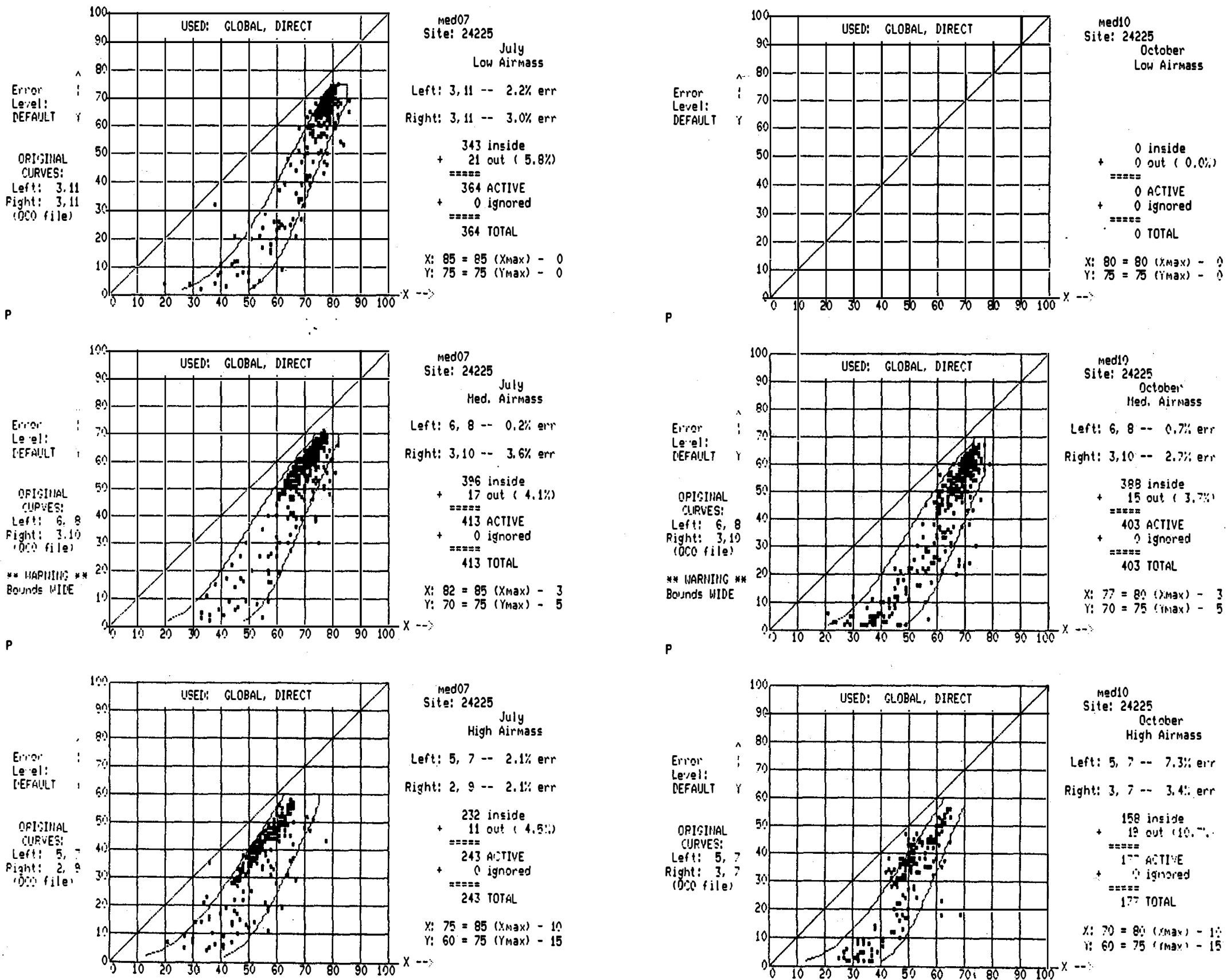

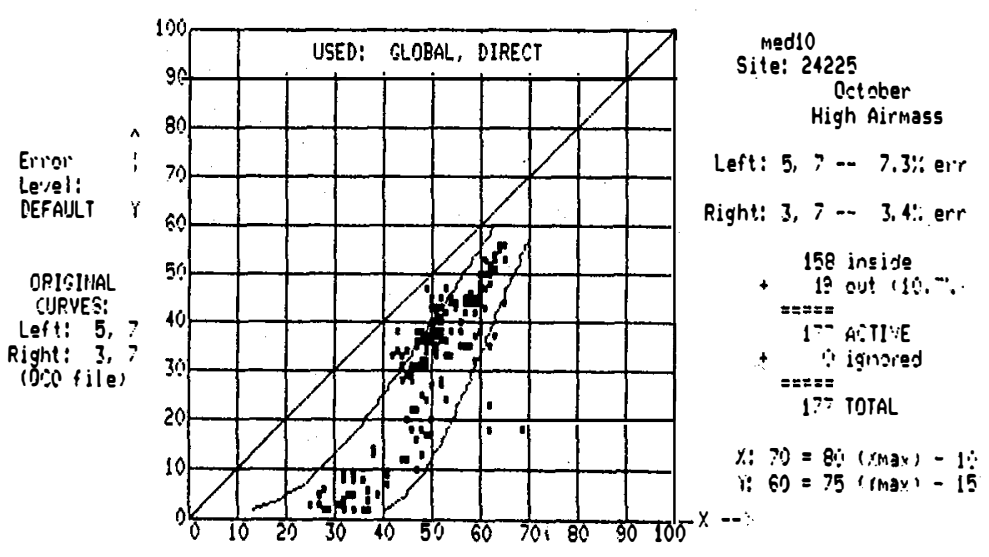


P

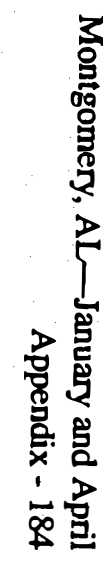

Error:

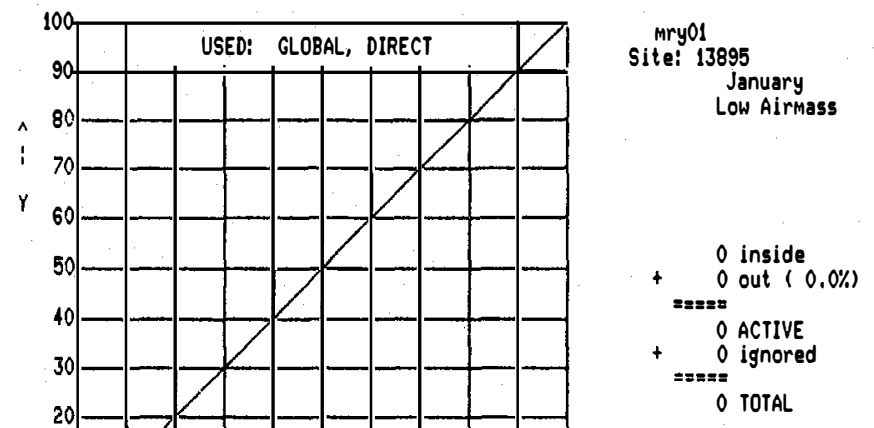

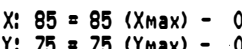
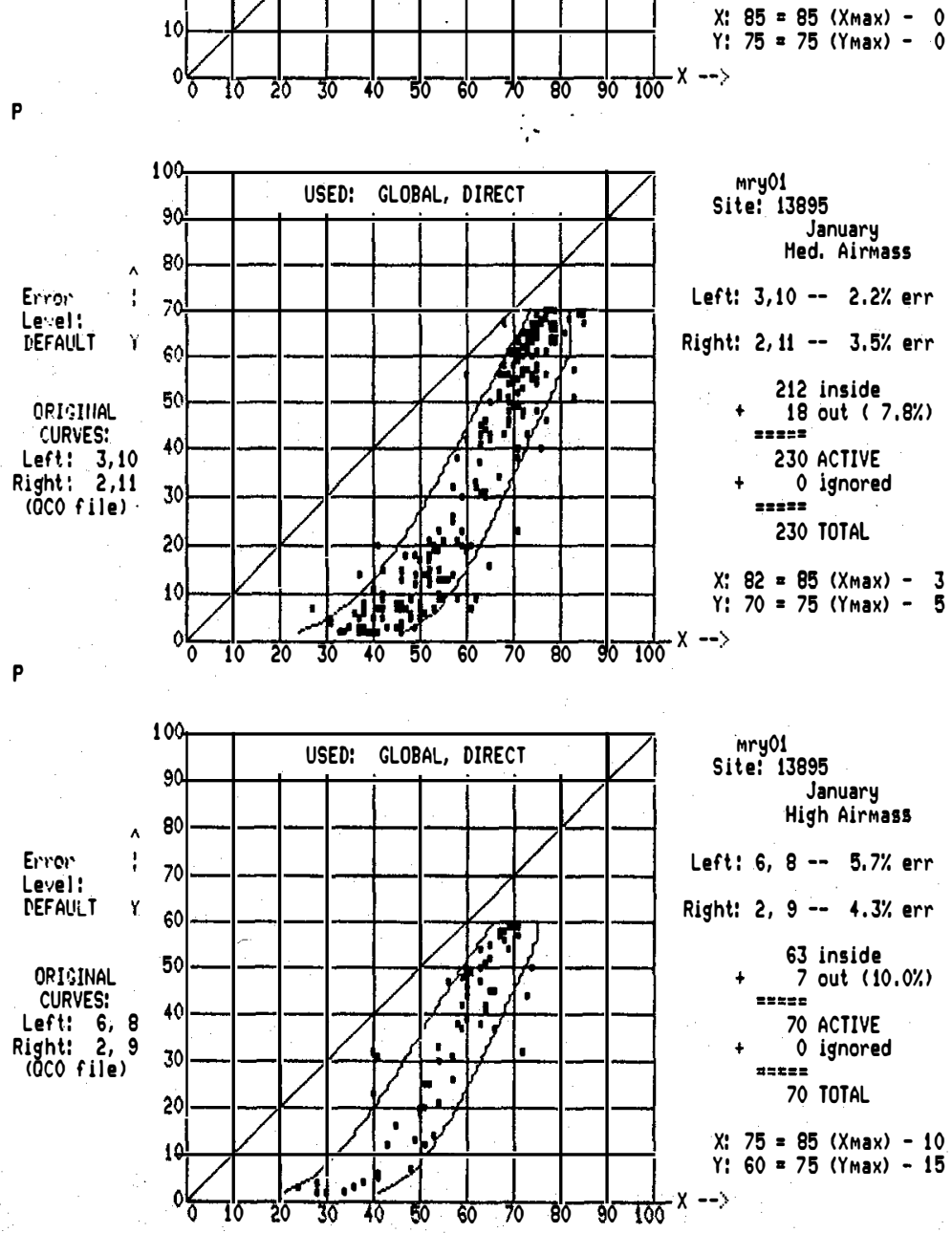
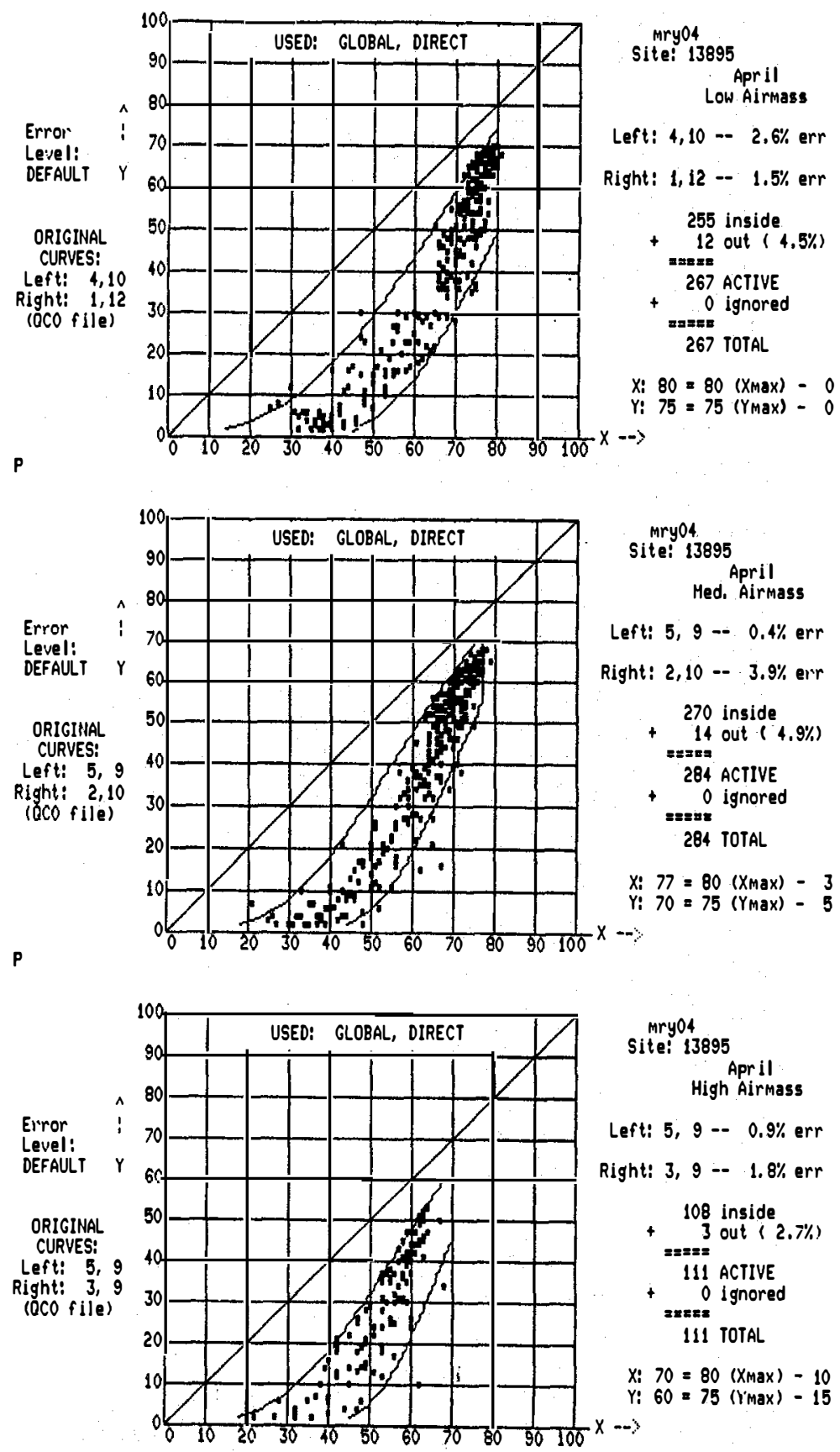


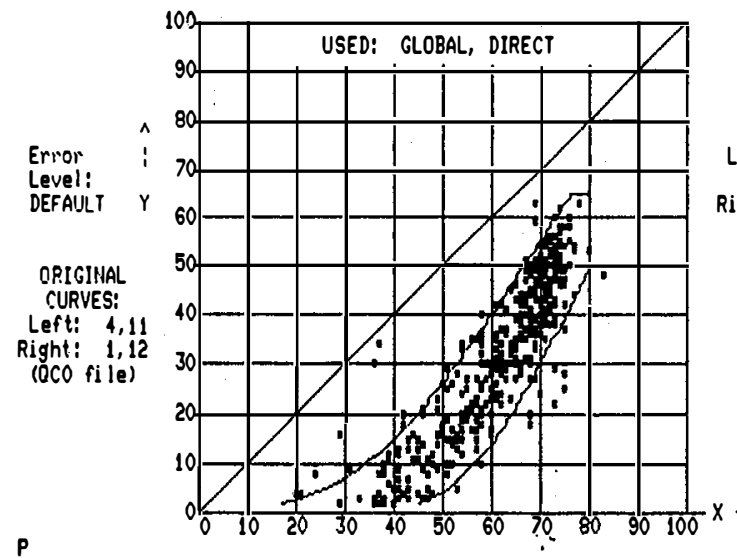

Site: 13895 July
Low Airmass

Left: $4,11-4.5 \% \mathrm{err}$

Right: $1,12-3.0 \%$ err

370 inside

=s=s=

401 ACIIVE
$+\quad 0$ ignored

$=2=2$
401 TOTAL

$X_{:} 80=80\left(X_{\max }\right)-0$ $\left(y_{\max }\right)-0$

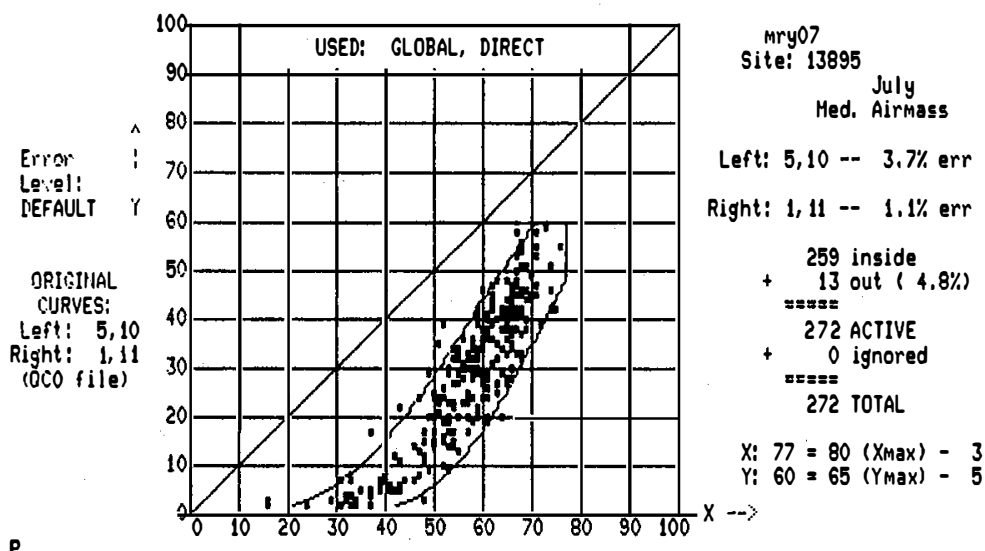

告

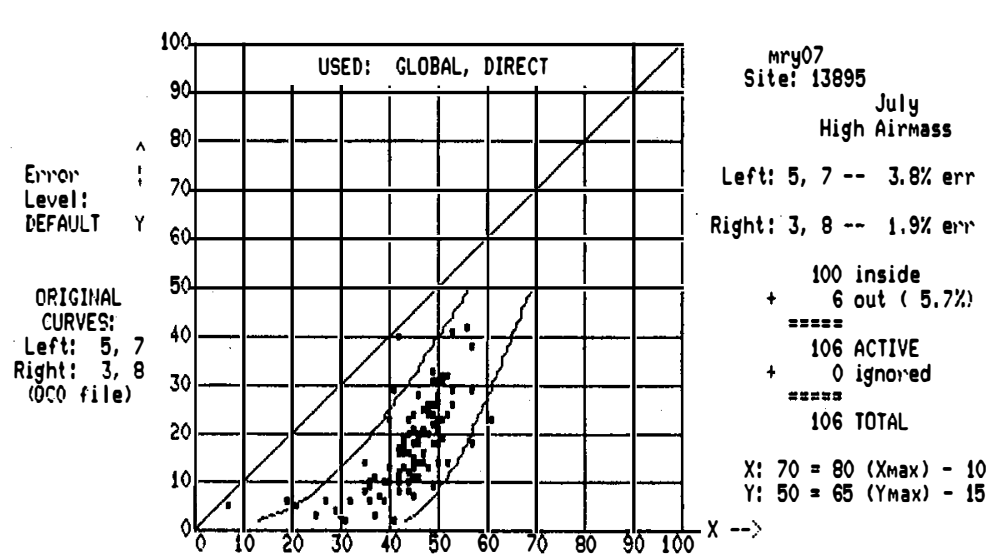

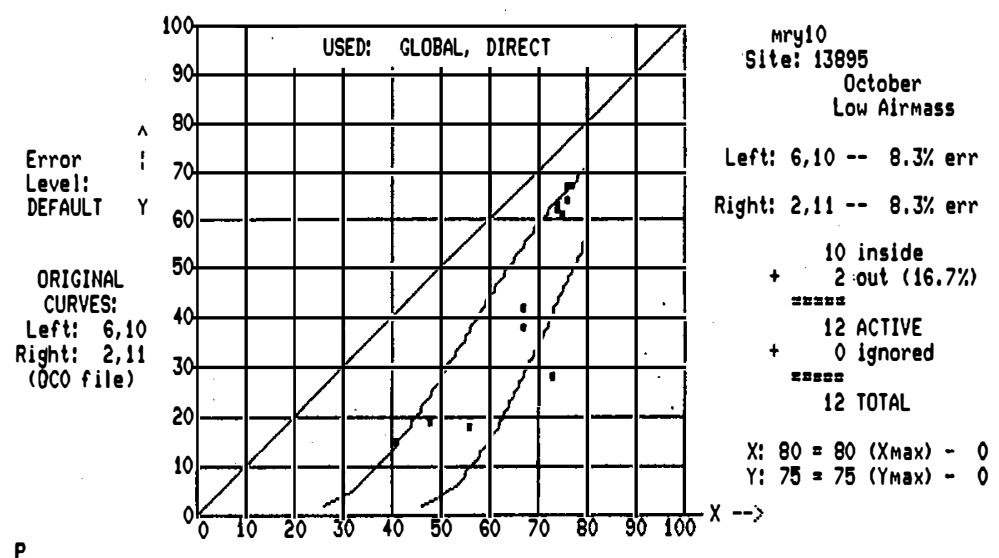
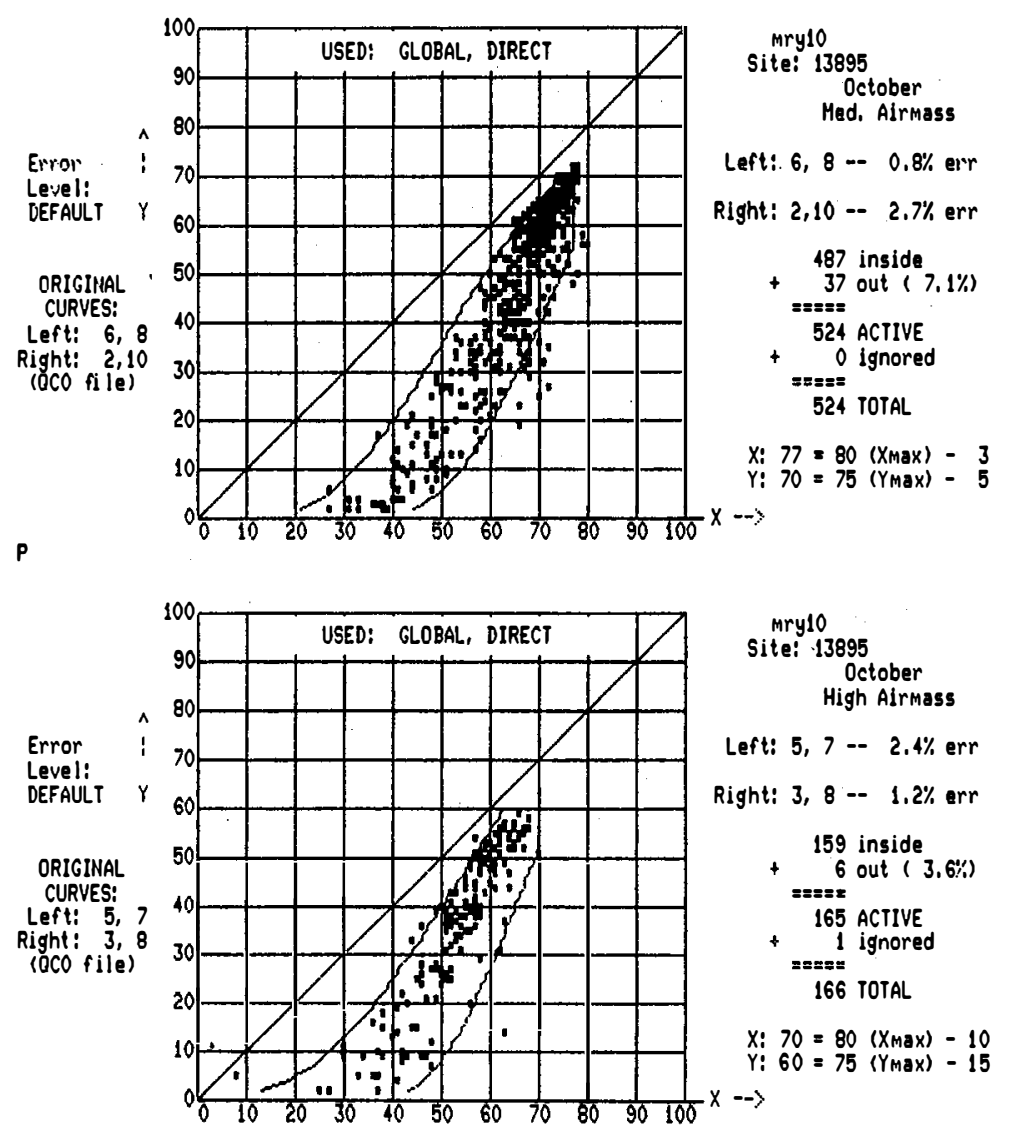

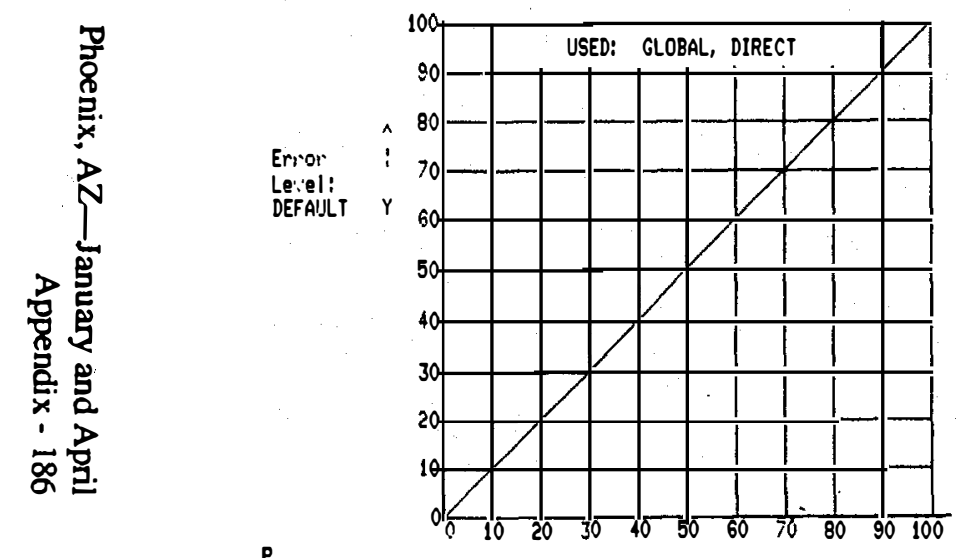

phxo1
Site: 23183

Lownuary
Low Airmass

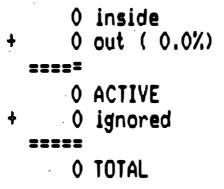

$X: 80=80\left(x_{\max }\right)=0$
$Y: 80=80\left(Y_{\max }\right)=0$

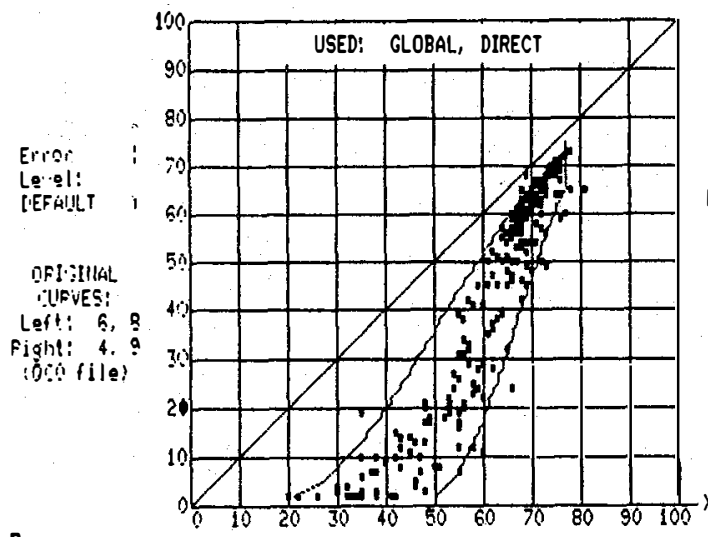

site: 2318 January
Med. Airmas Left: $6,8-2.1 \%$ err Right: 4, 9 -- 3.0\% err 219 inside
$+\quad 15$ out $(6.4 \%)$ 234 ACTIVE \begin{tabular}{l}
234 \\
$+\quad 0$ ignored \\
\hline
\end{tabular} 234 TOTAL

$X: 77=80\left(X_{\max }\right)-3$
$Y: 75=80\left(Y_{\max }\right)-5$ -

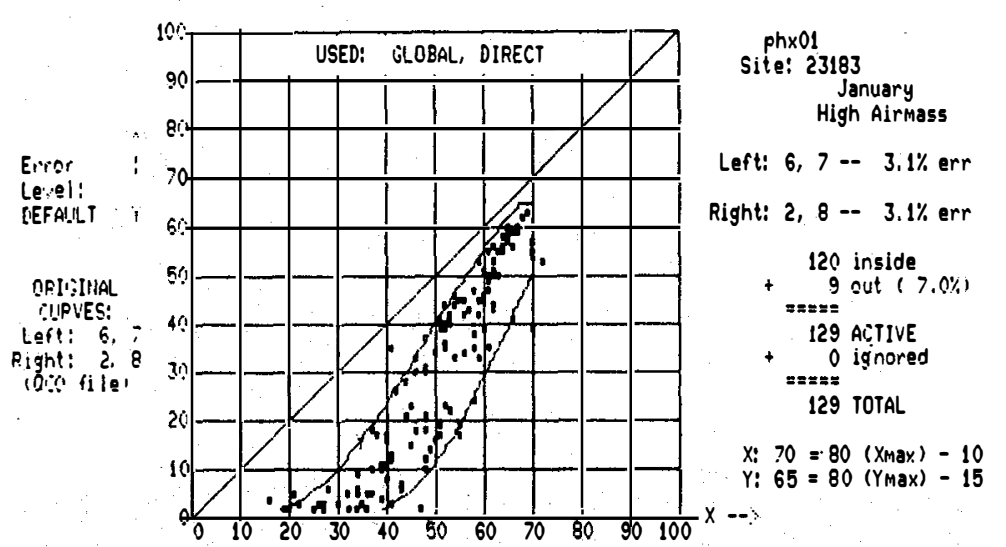

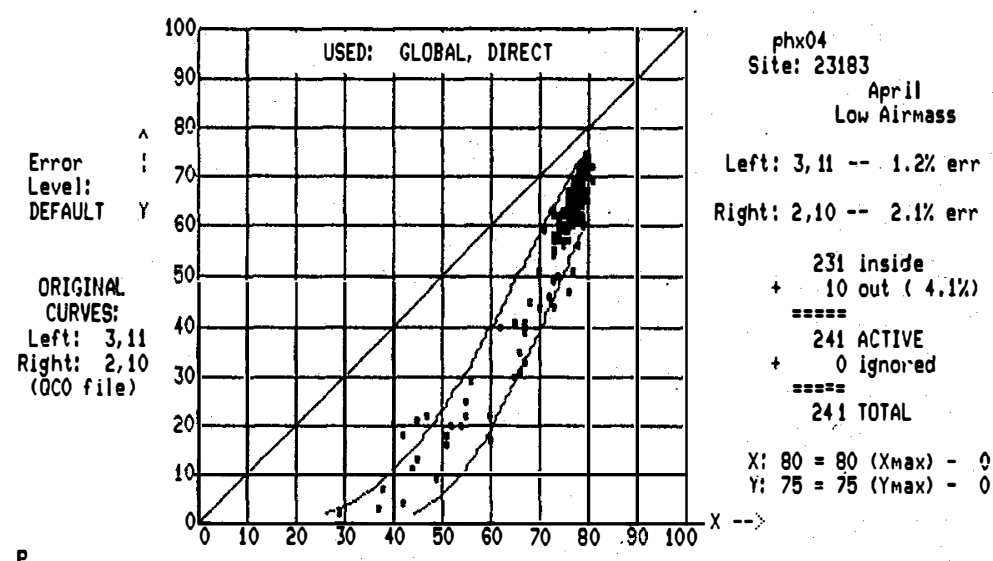
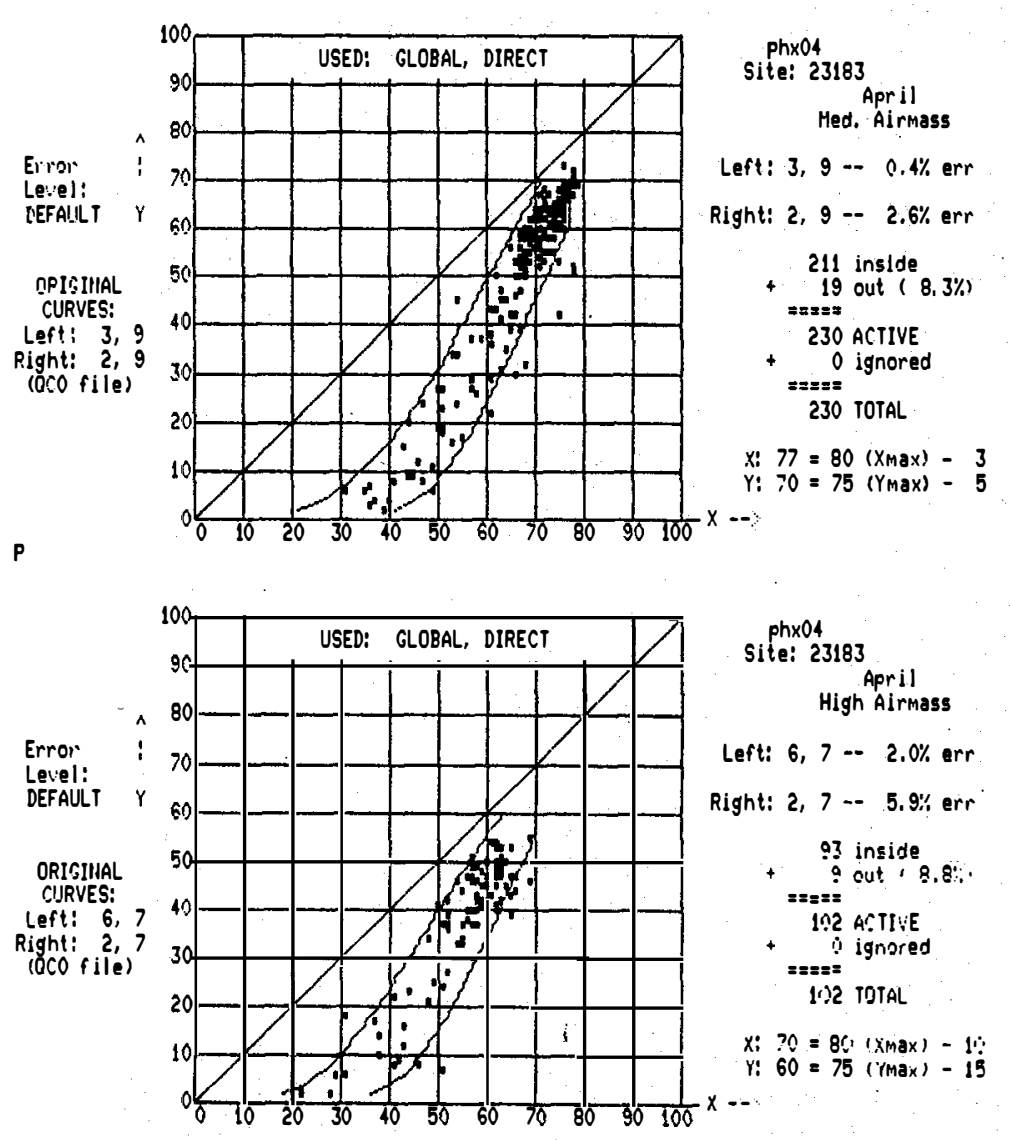


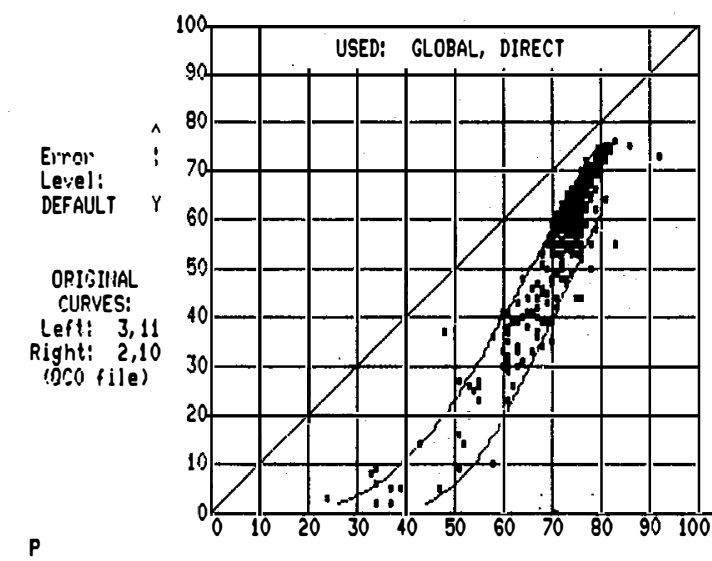

Site: 23183

Low Airmass

Left: $3,11-2 \quad 1.6 \% \mathrm{err}$

Right: 2,10 -- 2.2\% eir

417 inside
$+\quad 30$ out $(6.7 \%)$

$=\geq= \pm=$

$+\quad 0$ ignored

447 TOTAL

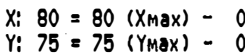
$75=75\left(Y_{\max }\right)-0$

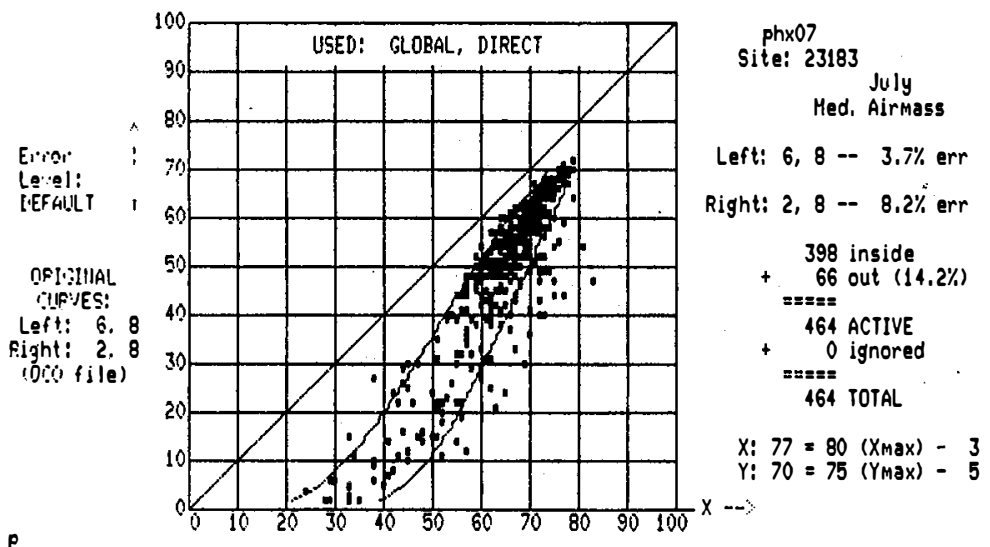

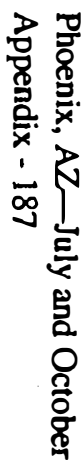

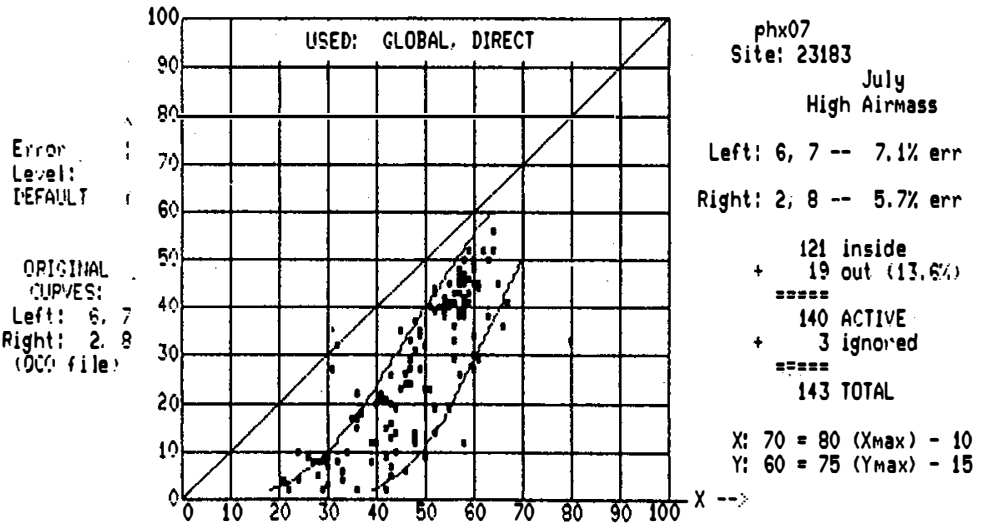

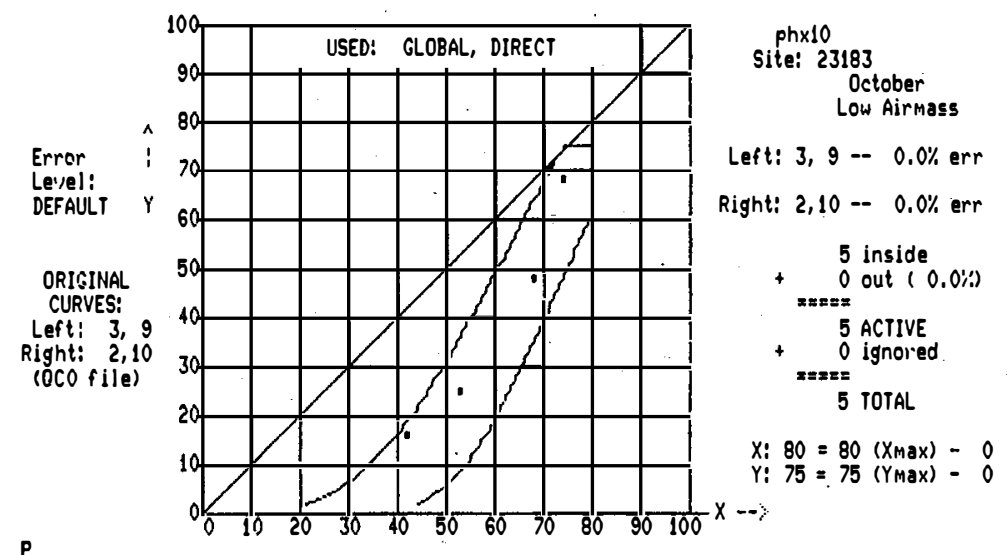
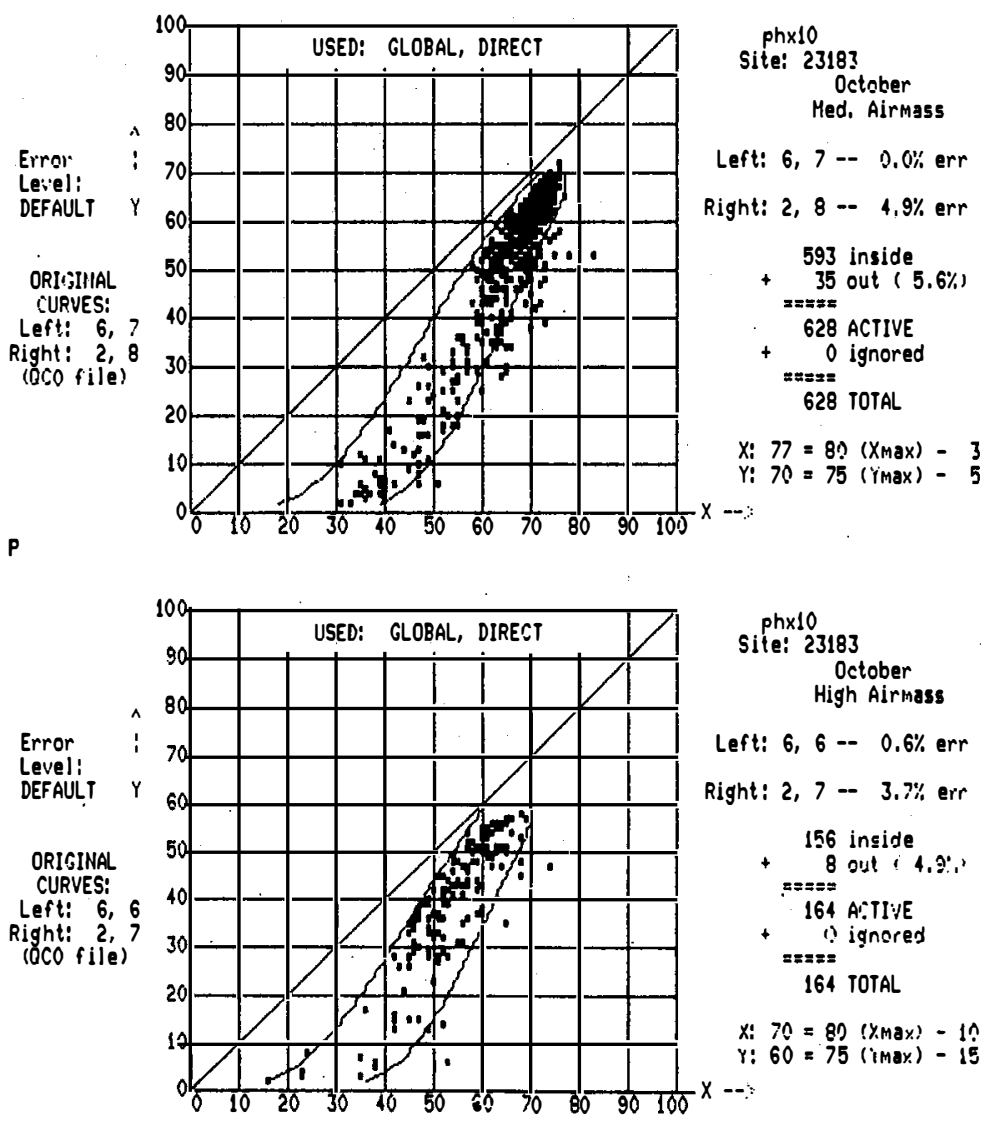


Error

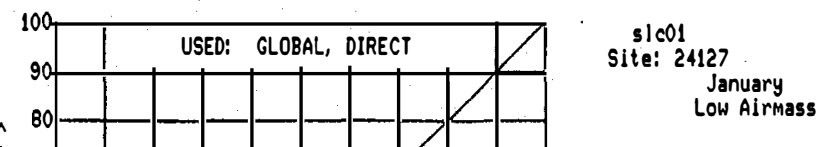

DEFAULT
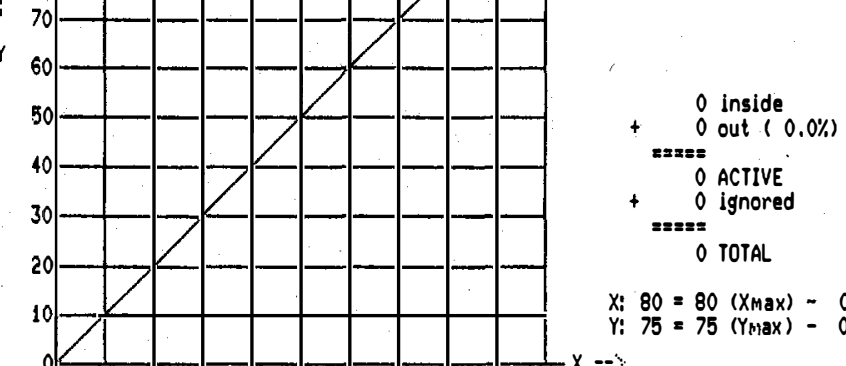
$X: 80=80\left(X_{\text {Max }}\right)=0$
$Y: 75=75\left(Y_{\max }\right)-0$
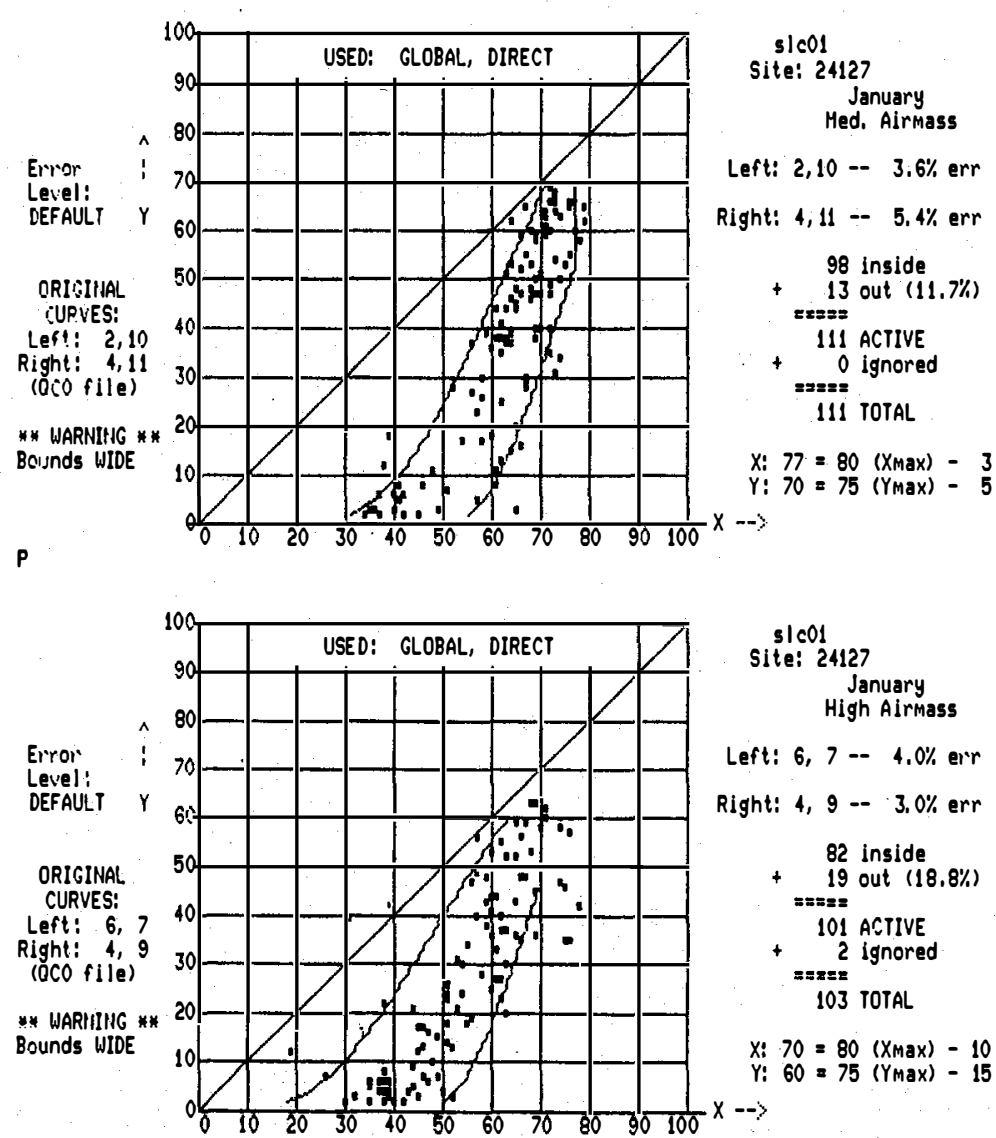
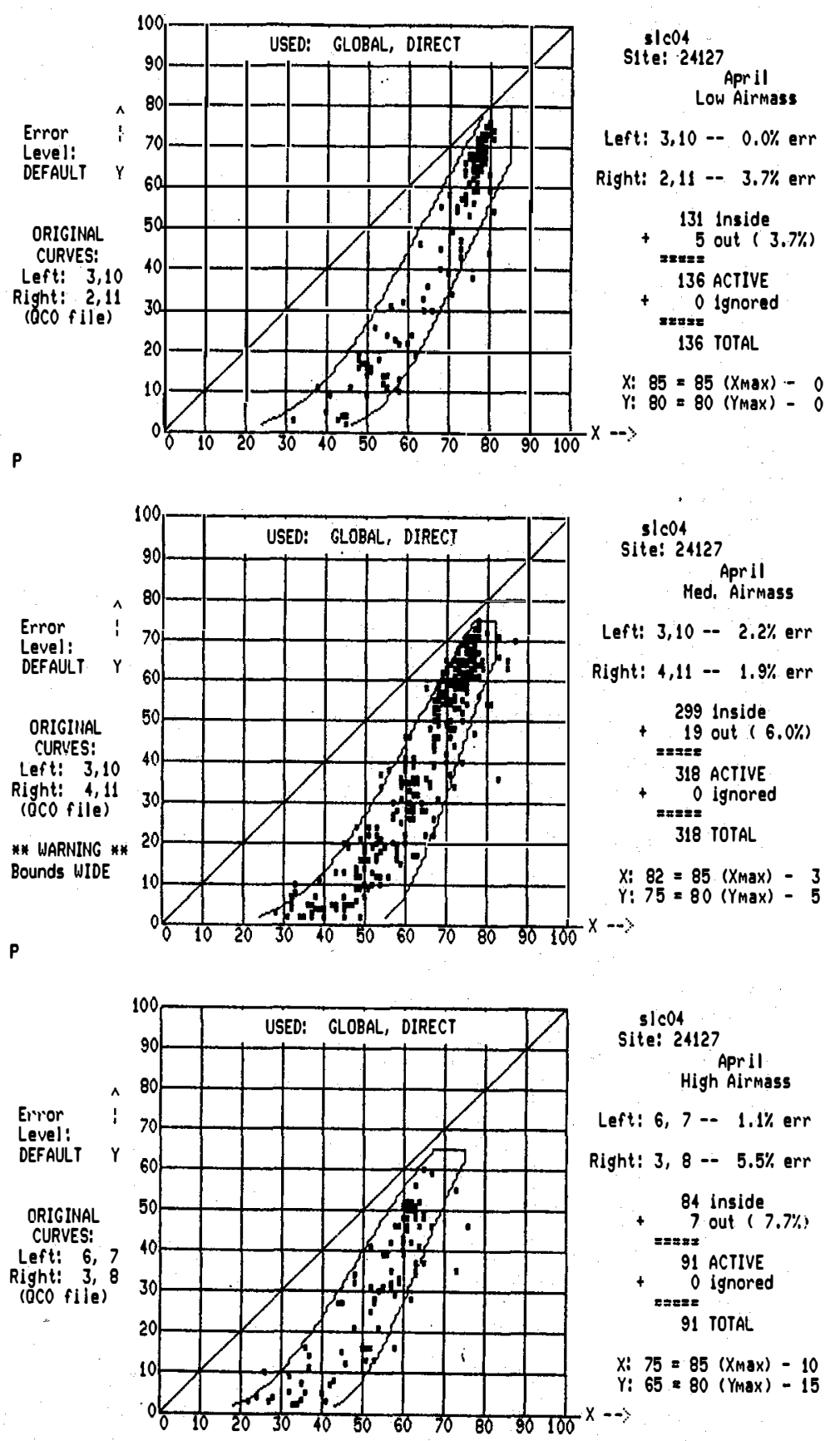

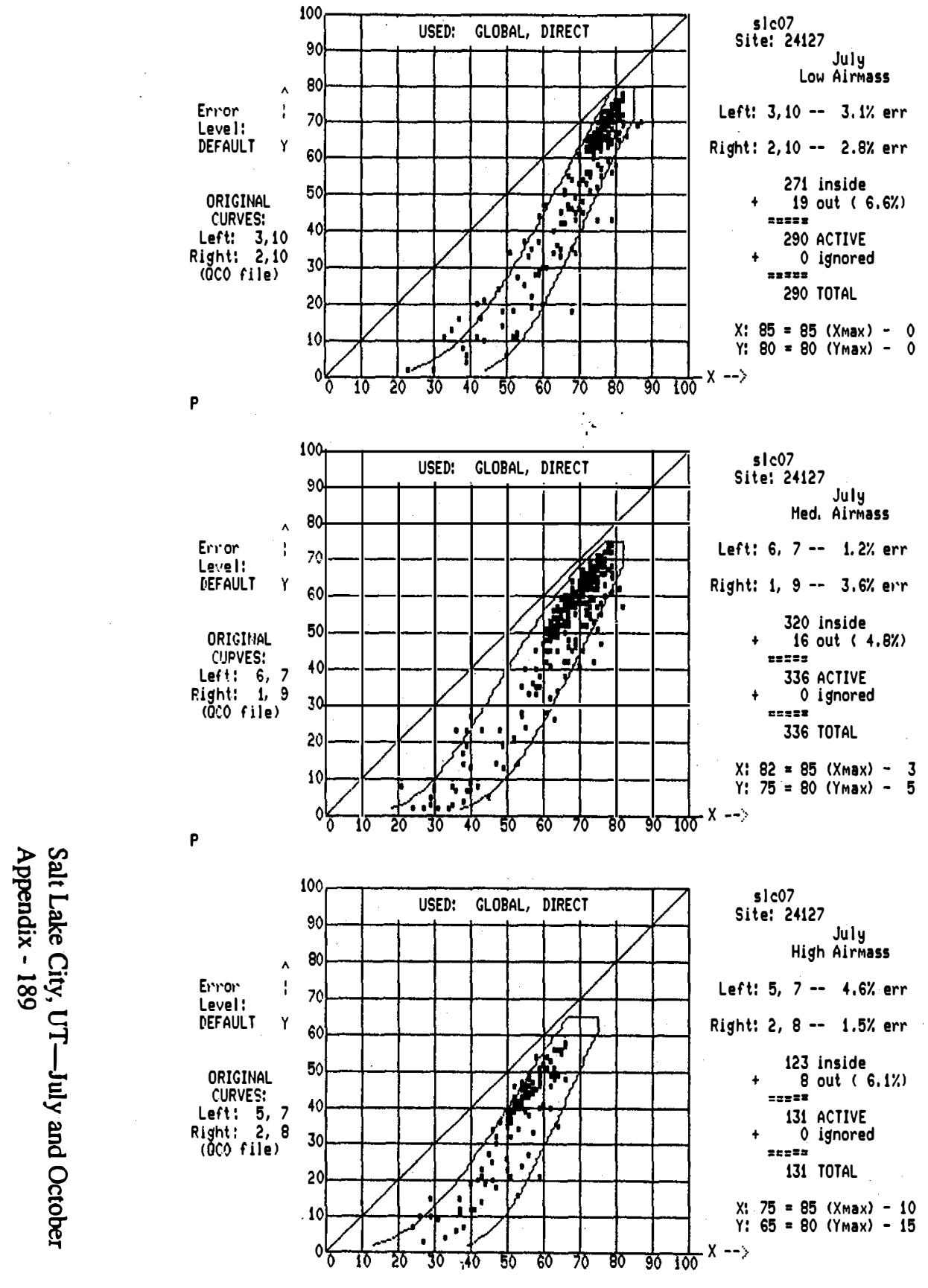
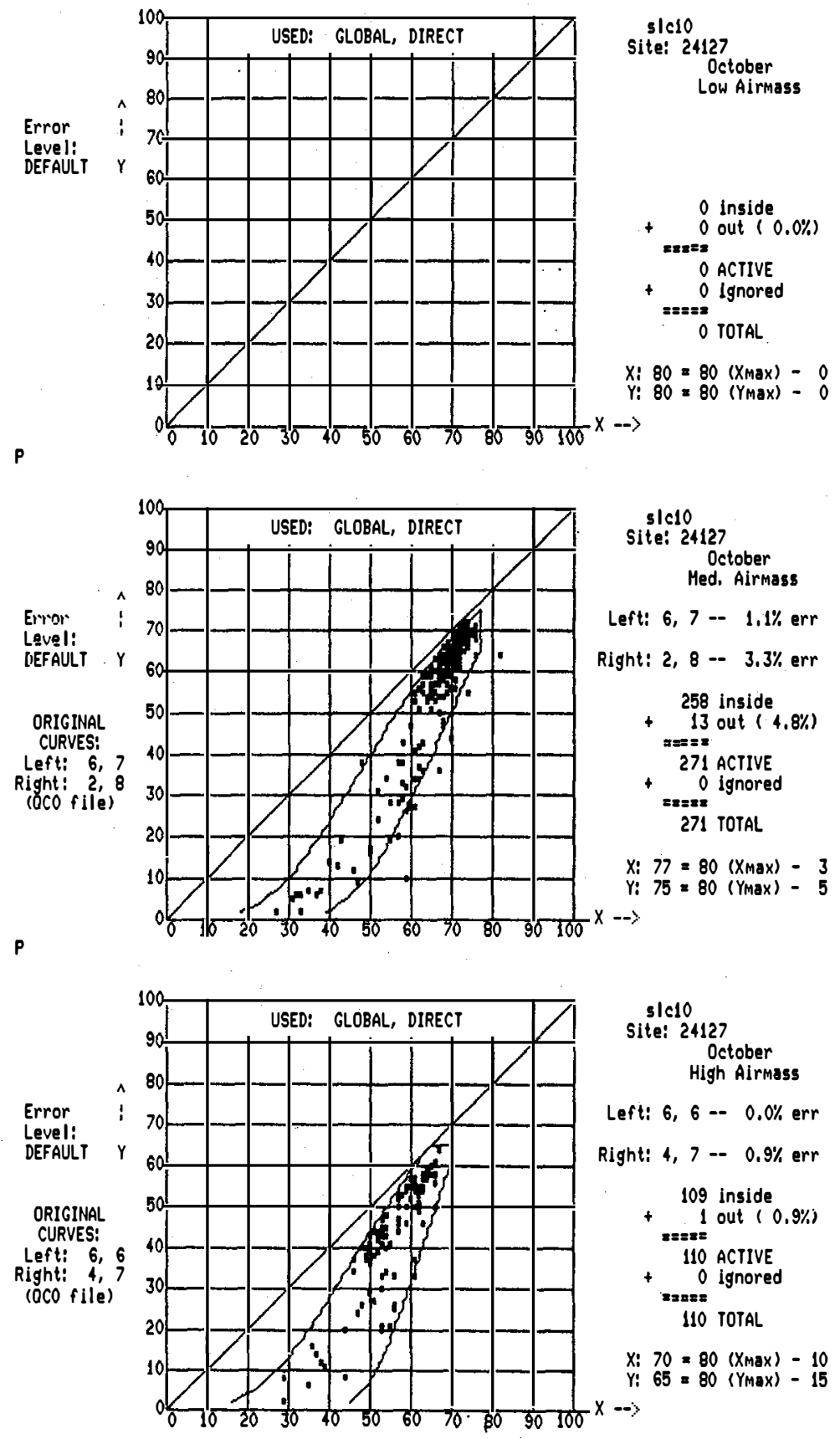


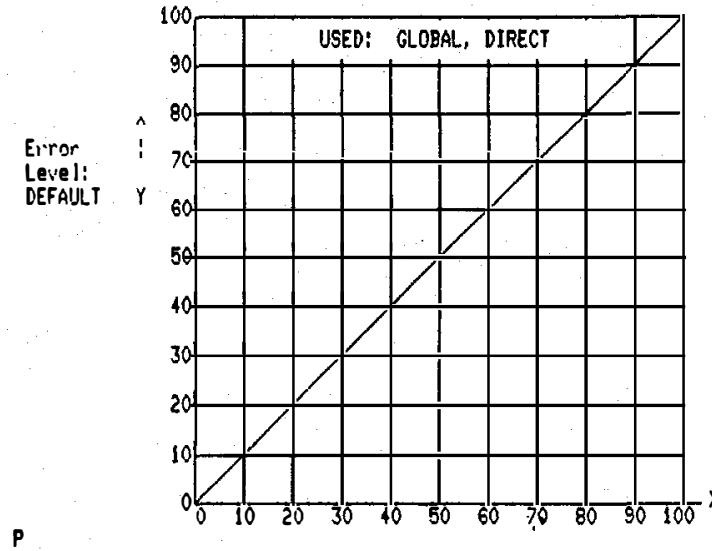

site: 93734

January

$\quad 0$ inside
$+\quad 0$ out $(0.0 \%)$

$==2$ ACTIVE

$+\quad 0$ ignored

0 TOTAL $X_{:} 80=80\left(X_{\max }\right)-0$
$Y: 75=75\left(Y_{\max }\right)-0$
$\left.X_{i}\right)-8$
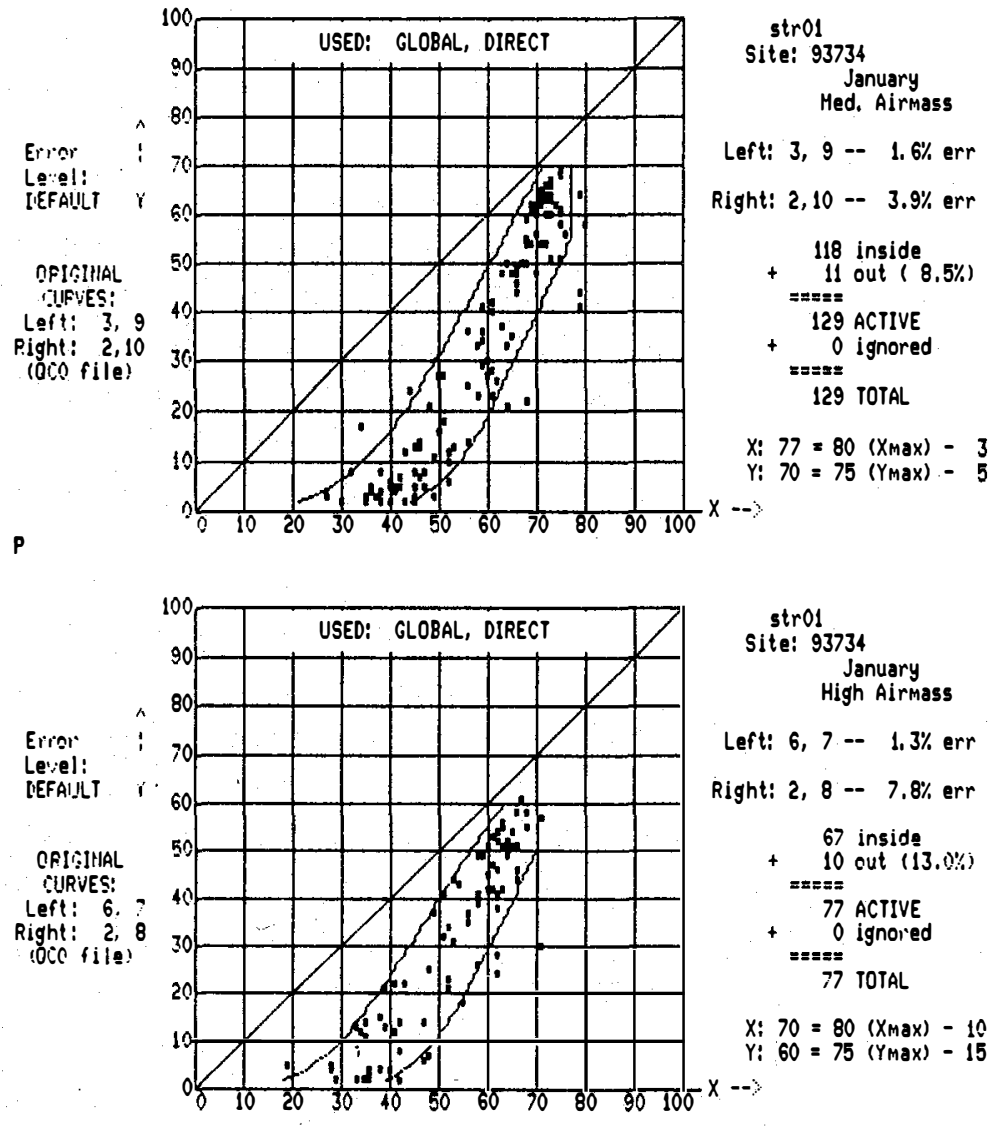

strol January
Med. Airmass Right: 2,10 -- 3.9\% err

118 inside
$+\quad 11$ out ( $8.5 \%)$ $=2== \pm$ ACTIVE 129 ACIVE
$+\quad 0$ ignored 129 TOTAL

$X: 77=80$ (Xmax) -3 Y: $70=75\left(Y_{\text {max }}\right)-5$

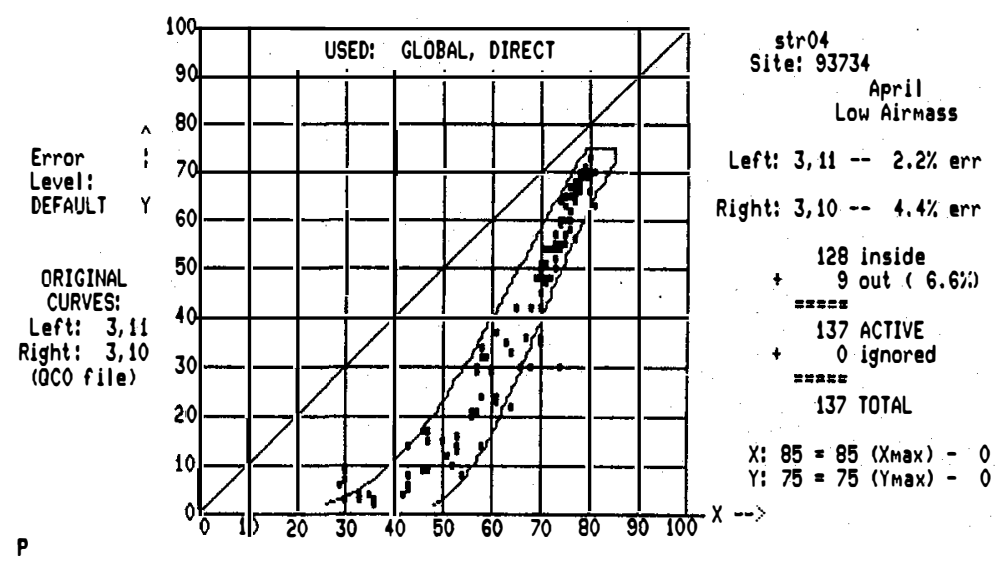

Left: $3,9--1.6 \% \mathrm{err}$

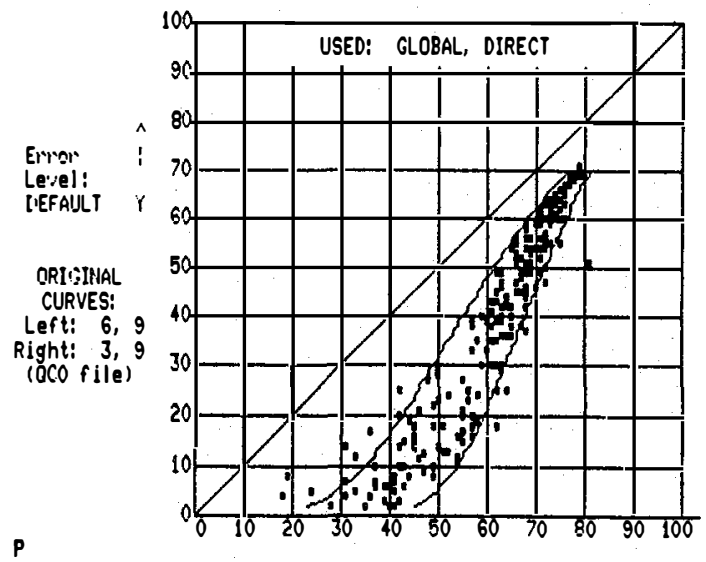

stro4
Site: 93734 April
Med. Airmass Left: $6,9--4,1 \%$ err Right: 3, 9 -- $4.1 \%$ err 202 inside
$+\quad 19$ out $(8.6 \%)$ ex==s out $(8.6 \%$ 221 ACTIVE
$+\quad 0$ ignored $=32 x$
221 TOTAL

$X: 82=85\left(X_{\text {max }}\right)-3$
$Y: 70=75\left(\right.$ (Yax $\left.^{\prime}\right)-5$

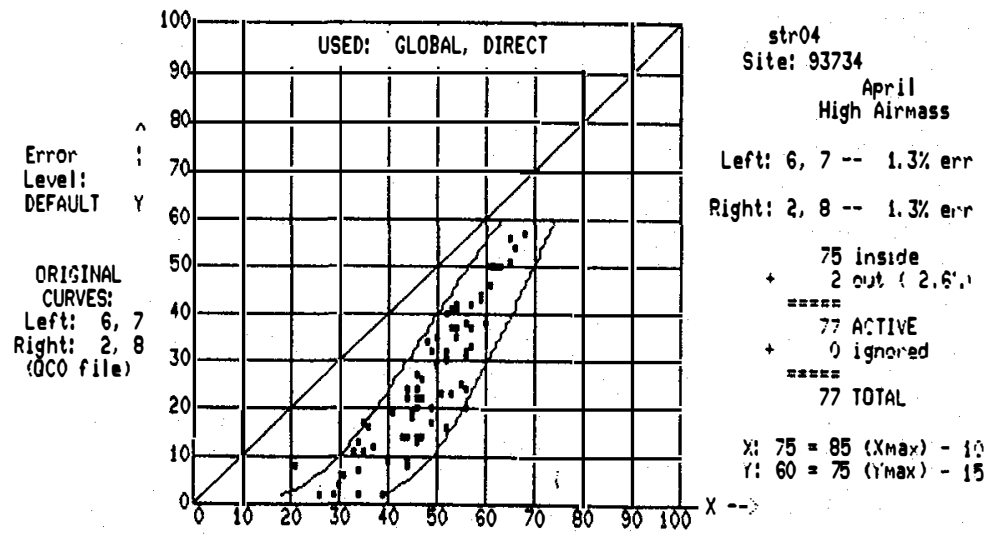




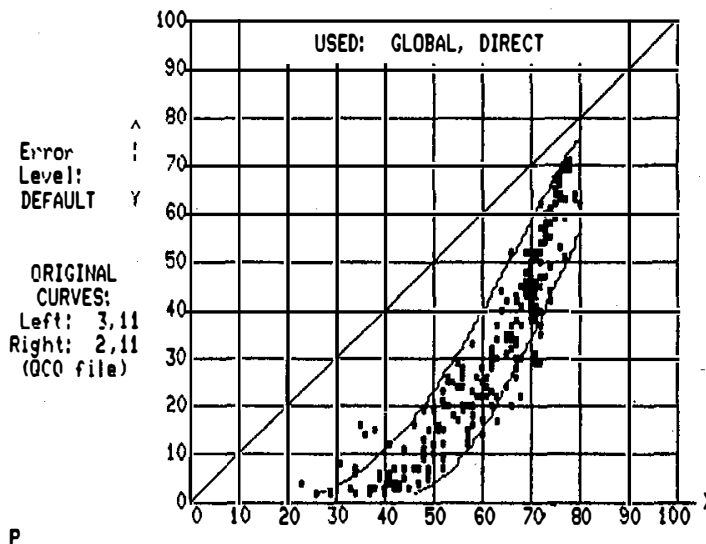

str07

Site: 93734 Low Airmass Left: $3,11=3.6 \% \mathrm{err}$ Right: 2,11 -- $5.2 \% \mathrm{err}$ 229 inside
$+\quad 22$ out $(8.8 \%)$ =es=s $+\quad 0$ ignored 251 TOTAL

$X: 80=80\left(X_{\text {max }}\right)-0$ $Y: 75=75\left(Y_{\text {Max }}\right)-0$

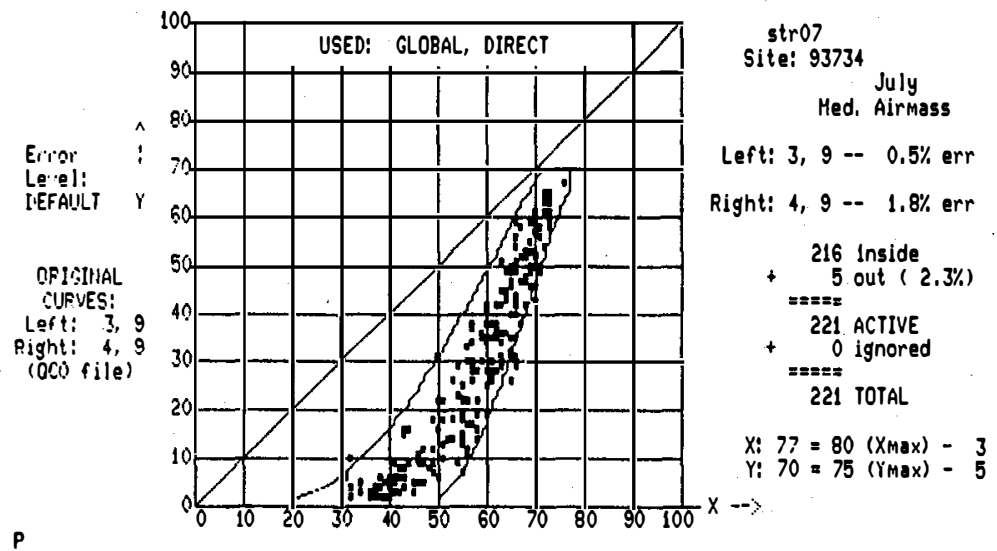

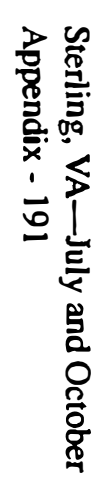

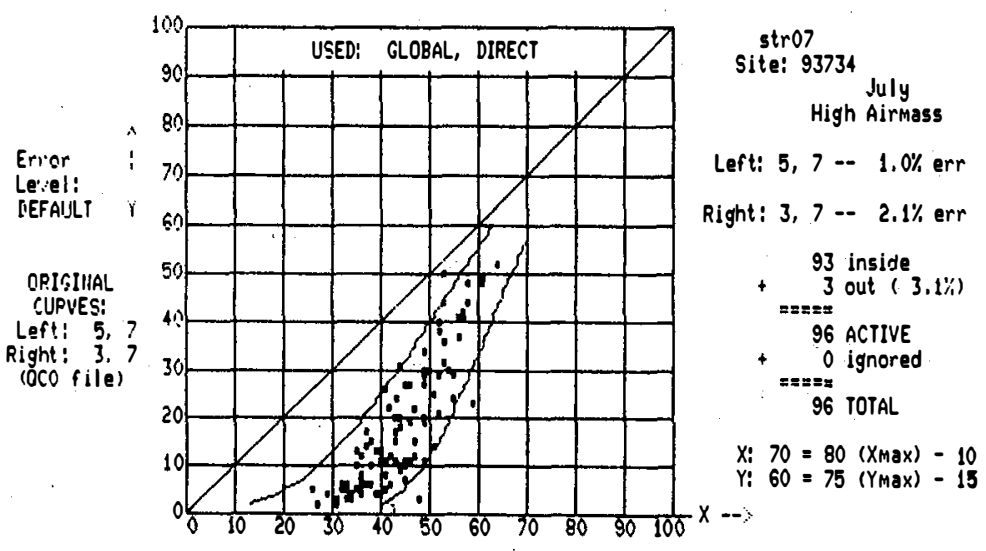

str10
Site: 93734 Low Airmass

Error:

DEFAULT

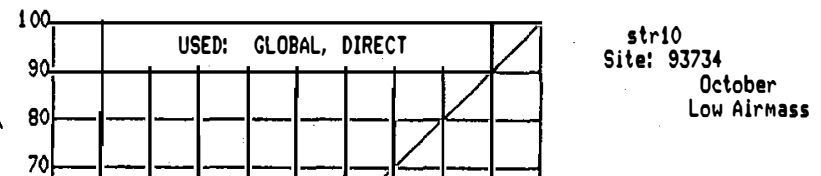

0 inside
$+\quad 0$ out $(0.0 \%)$

= $=8$ active

$+\quad 0$ ignored

0 TOTAL

$X_{:} 80=80\left(X_{\max }\right)-$ Y!
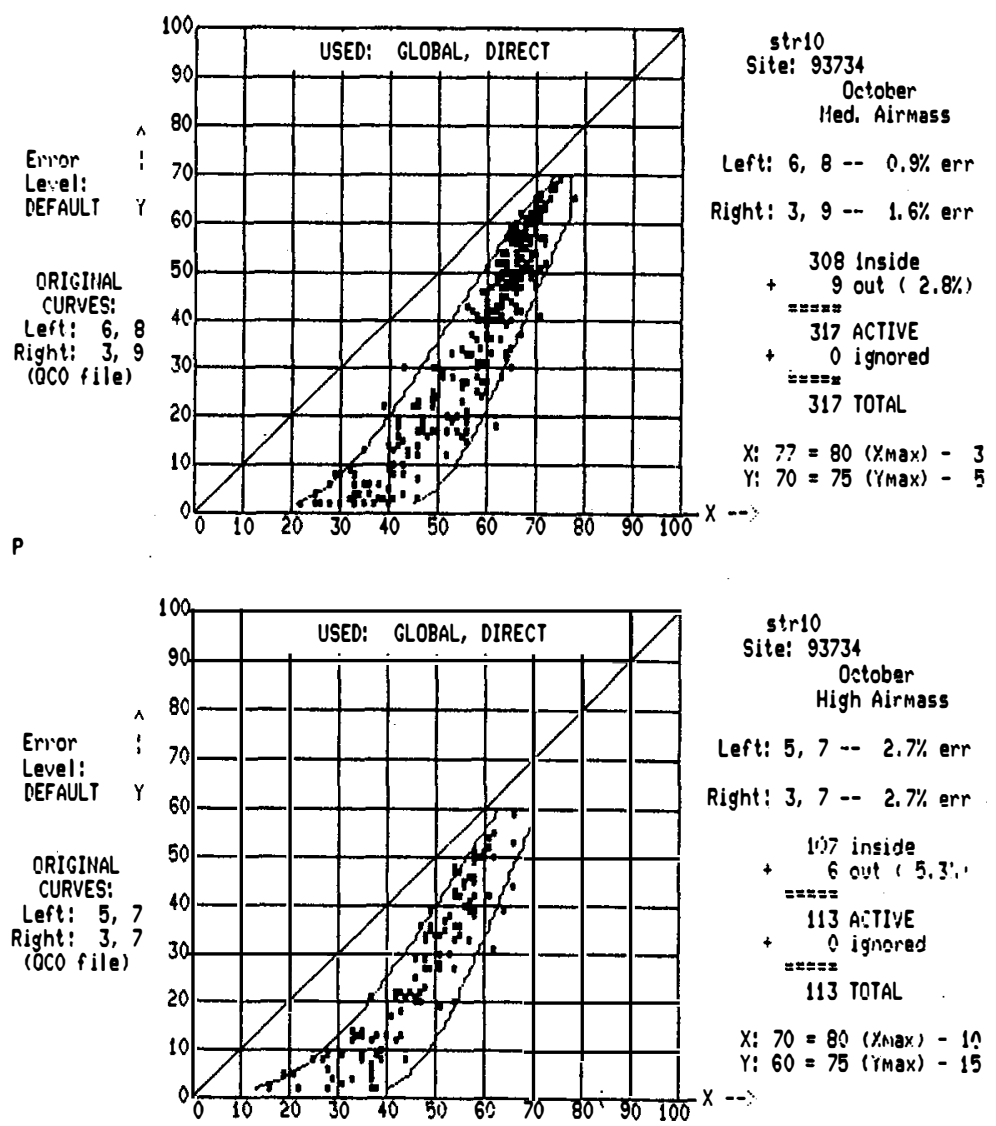


\begin{tabular}{|c|c|c|c|}
\hline $\begin{array}{c}\text { Document Control } \\
\text { Page }\end{array}$ & $\begin{array}{l}\text { 1. NREL Report No. } \\
\text { NREL-TP-463-5608 }\end{array}$ & $\begin{array}{l}\text { 2. NTIS Accession No. } \\
\text { DE93018210 }\end{array}$ & 3. Recipient's Accession No. \\
\hline \multirow{2}{*}{\multicolumn{3}{|c|}{$\begin{array}{l}\text { 4. Title and Subtitle } \\
\text { Users Manual for SERI QC Software-Assessing the Quality of Solar } \\
\text { Radiation Daw }\end{array}$}} & $\begin{array}{l}\text { Publication Date } \\
\text { December } 1993\end{array}$ \\
\hline & & & 6. \\
\hline \multicolumn{3}{|c|}{$\begin{array}{l}\text { 7. Author(s) } \\
\text { E. Maxwell, S. Wilcox, and Marin Rymes }\end{array}$} & 8. Performing Organization Rept. No. \\
\hline \multirow{2}{*}{\multicolumn{3}{|c|}{$\begin{array}{l}\text { 9. Performing Organization Name and Address } \\
\text { National Renewable Energy Laboratory } \\
1617 \text { Cole Boulevard } \\
\text { Golden, Colorado } 80401-3393\end{array}$}} & $\begin{array}{l}\text { 10. Project/TaskWork Unit No. } \\
\text { RA310104 and RA411040 }\end{array}$ \\
\hline & & & $\begin{array}{l}\text { 11. Contract (C) or Grant (G) No. } \\
\text { (C) } \\
\text { (G) }\end{array}$ \\
\hline \multirow{2}{*}{\multicolumn{3}{|c|}{ 12. Sponsoring Organization Name and Address }} & $\begin{array}{l}\text { 13. Type of Report \& Period Covered } \\
\text { Software Users Manual }\end{array}$ \\
\hline & & & 14. \\
\hline \multicolumn{4}{|l|}{ 15. Supplementary Notes } \\
\hline \multicolumn{4}{|c|}{$\begin{array}{l}\text { 16. Abstract (Limit: } 200 \text { words) } \\
\text { This users manual provides general infornation on solar radiation measurements and quality control measures and } \\
\text { options. It was written for the user of SERI QC, a software package developed at the National Renewable Energy } \\
\text { Laboratory (formerly the Solar Energy Research Instititue [SERI) for performing quality assessments of data } \\
\text { obtained from one-minute to one-hour integrations of solar radiation; it is not applicable for performing quality } \\
\text { assessents of daily total solar radiation energy. The software is described, along with the theory encompassing its } \\
\text { appproach to postmeasurement quality assessment. Examples of typical measurement problems are given. }\end{array}$} \\
\hline \multirow{2}{*}{\multicolumn{4}{|c|}{$\begin{array}{l}\text { 17. Document Analysis } \\
\text { a. Descriptors } \\
\text { Solar radiation; data assessment; software; solar radiation measurements } \\
\text { b. Identifiers/Open-Ended Terms }\end{array}$}} \\
\hline & & & \\
\hline \multirow{2}{*}{\multicolumn{2}{|c|}{$\begin{array}{l}\text { 18. Availability Statement } \\
\text { National Technical Information Service } \\
\text { U.S. Department of Commerce } \\
\text { 5285 Port Royal Road } \\
\text { Springfield, VA } 22161\end{array}$}} & & $\begin{array}{l}\text { 19. No. of Pages } \\
202\end{array}$ \\
\hline & & & $\begin{array}{l}\text { 20. Price } \\
\text { A10 }\end{array}$ \\
\hline
\end{tabular}

Form No. 0069E (6-30-87) 